\title{
Software for combinatorial power series
}

\author{
by
}

\author{
Andrew MacFie
}

A thesis submitted to the Faculty of Graduate and Post Doctoral Affairs in partial fulfillment of the requirements for the degree of

\author{
Master of Science \\ in \\ Pure Mathematics
}

Carleton University, Ottawa, Canada

(C) 2012

Andrew MacFie 


\begin{abstract}
Generating functions (i.e. power series) have applications throughout enumerative and analytic combinatorics. In this thesis we present Genfunlib, a new Mathematica package containing a selection of implementations of symbolic methods related to generating functions. With Genfunlib one can find the generating functions for regular languages, compute the initial terms of a generating function, convert between generating function equations and recurrences, and find asymptotics. This thesis gives mathematical background, extensive documentation for Genfunlib, and tutorials.
\end{abstract}




\section{Acknowledgements}

I would first of all like to acknowledge David Thomson for introducing me to my supervisor for this thesis, Daniel Panario, in 2009. Professor Panario supervised me on a number of projects between then and now, and I especially want to thank him for his guidance on this thesis. Without his experience and the wisdom he shared, this project would not have been a success. I appreciate all of his work with me greatly.

I would also like to thank Brett Stevens for advising me during initial development of the software part of this thesis, in 2012. Professor Stevens also provided encouragement and feedback during the early planning stages of this thesis.

I am grateful for the input, ideas, and suggestions of Bruno Salvy, whom Professor Panario helpfully introduced me to in 2012.

I thankfully acknowledge the highly useful comments I received from the members of the thesis defense committee: Zhicheng Gao, Pat Morin, and Mike Newman.

Thank you all. 


\section{Contents}

1 Introduction 1

2 Mathematics for the analysis of algorithms 3

$\begin{array}{lll}3 & \text { A few notes on Mathematica } & 7\end{array}$

4 Guide to Genfunlib 10

4.1 Power series and their coefficients . . . . . . . . . . . . . . 10

4.2 Numerical coefficients . . . . . . . . . . . . . . 16

4.3 Regular languages . . . . . . . . . . . . . . . . . 20

4.4 Symbolic method . . . . . . . . . . . . . . . . . . 36

4.5 Asymptotics . . . . . . . . . . . . . . . . . . 42

5 Genfunlib in action $\quad 46$

5.1 Mathematics contest problems . . . . . . . . . . . 46

5.2 Miscellaneous problems . . . . . . . . . . . . . . 67

$\begin{array}{lll}6 & \text { Further work } & 74\end{array}$

$\begin{array}{ll}\text { References } & 75\end{array}$

$\begin{array}{ll}\text { Appendix } & 79\end{array}$ 


\section{Introduction}

According to the NSF, a scientist's worth is not dependent solely on publications. ... In the next five years, I believe that it will become routine to track - and to value-citations to an online lab notebook, contributions to a software library, bookmarks to data sets from content-sharing sites such as Pinterest and Delicious.

- Heather Piwowar, 2013 [27]

This thesis is on Genfunlib, a package for the Mathematica computer algebra system, which was written by the author of this document. The goal of Genfunlib is to provide commands for obtaining and using generating functions to solve combinatorial problems. The full source code of Genfunlib can be found in the Appendix; it is also available on the web at this address:

https://github.com/amacfie/Genfunlib

Genfunlib consists of implementations of a number of well-known procedures not currently available in Mathematica or any third-party Mathematica package. Genfunlib includes comprehensive finite automata functionality that allows the user to convert between automata and regular expressions, and perform standard operations such as union, intersection, and reversal. Generating functions that count the number of words in a given regular language can be computed. These generating functions can be useful for counting problems that involve regular languages such as pattern avoidance in words. Genfunlib uses known methods to produce asymptotic information about a counting sequence given its generating function. From a power series equation, Genfunlib can compute the initial terms of the solution series. Genfunlib improves on Mathematica's built-in capabilities for converting between recurrence relations and power series equations. The symbolic method for obtaining generating functions 
from constructible combinatorial classes is also implemented in Genfunlib.

The contents of this thesis are as follows. In Section 2, we give an overview of enumerative combinatorics, generating functions, and their applications to analysis of algorithms. Thanks to this section, the mathematical content of this thesis is fairly self-contained. Section 3 is a brief overview of Mathematica programming to allow readers to better understand the code in this thesis. Section 4 goes through each of Genfunlib's commands and explains how to use them, what they do and, where educative, how they do it. It is divided into subsections that cover all the commands related to one mathematical topic. This section can be considered to be both user and developer documentation. Boxes labeled "Mathematica note" can be ignored by readers unfamiliar with Mathematica. In Section 5 we present a number of relatively elementary combinatorial problems, and solutions that make use of Genfunlib and Mathematica. In the solutions, we attempt to give a sense of the range of Genfunlib's functionality and try to minimize the number of operations performed "by hand". The problems in Section 5.1 come from high school mathematics contests, or contest preparation material. Finally, in Section 6 we conclude by offering possible paths for further work.

Naturally, Genfunlib does not implement all known algorithms that one may want to use with generating functions. Areas for expansion and improvement are discussed as they are relevant at various points in the body of the thesis. Further, there are tests and operations on power series for which no algorithm has been found, e.g. whether a given univariate rational function has only positive coefficients [16]. 


\section{Mathematics for the analysis of algorithms}

In theoretical computer science, the greatest innovation is the realisation that algorithms are mathematical objects, and can be rigorously analysed in terms of their consumption of scarce resources, including space, time, and randomness.

- Jeffrey Shallit, 2006 [33]

Given a computer program, one may desire to know how long it will take to run in absolute terms, that is, in physical units of time. Indeed ultimately, such concrete predictions are always the end goal, and engineering methods involving statistics, simulation, and detailed knowledge of particular computing environments are certainly used (when rigorous this is known as the experimental analysis of algorithms). However, given the complexity of actual computers, a more abstract problem is taken to be the typical one in the field of computer science called analysis of algorithms.

First, instead of executable computer programs, the objects of study are algorithms [13], generally given at a level of abstraction where pseudocode is sufficient. The physical running time of an algorithm is not defined, but the number of steps it takes is. It is assumed that the number of steps taken by an algorithm gives information related to actual running time of computer implementations. From now on, the "running time" of an algorithm means the number of steps it takes.

Second, because algorithms are abstract objects and do not run on computers, the goal is not to predict absolute timings, but to compare algorithms with each other. Let $T(A, i)$ be the running time of an algorithm $A$ on an input $i$. Let $I(n)$ be the set of inputs with size $n$. We can represent the running time of 
$A$ with respect to a probability mass function $f_{n}: I(n) \rightarrow[0,1]$ by the sequence

$$
\left(T_{A}(n)\right), \text { where } T_{A}(n)=\sum_{i \in I(n)} T(A, i) f_{n}(i) .
$$

That is, $T_{A}(n)$ is the expected running time of $A$ according to the probability distribution defined by $f_{n}$. The most common distribution is "worst-case", which assigns probability 1 to an input in $I(n)$ on which the running time of $A$ is maximal. Another common distribution used is "average-case", where

$$
f_{n}(i)=1 /|I(n)|
$$

for all $i$. More nuanced analysis has been done beyond these two distributions, notably smoothed analysis and analysis using the Solomonoff-Levin measure [19].

Once the distribution has been chosen, for two algorithms $A$ and $B$ computing the same function, we have the two sequences $\left(T_{A}(n)\right)$ and $\left(T_{B}(n)\right)$. Obviously, if $T_{A}(n) \geq T_{B}(n)$ for all $n$, it is easy to compare $A$ and $B$, but this ordering is not always enough. Instead, analysis of algorithms uses asymptotics: relations are established between $T_{A}(n)$ and $T_{B}(n)$ as $n$ approaches infinity. Now, instead of directly comparing $T_{A}(n)$ to $T_{X}(n)$ for each $X$, we can, analogously to the "interlingua" approach to machine translation [15], find a relation between $T_{A}(n)$ and a simple standard sequence, after which, to compare algorithms we need only compare simple standard sequences, which is easy.

Definition 1 (Asymptotic notation). As $n \rightarrow \infty$,

- $f(n)=O(g(n))$ means $\exists c>0, N>0: \forall n, n>N \Longrightarrow f(n)<c g(n)$,

- $f(n)=\Omega(g(n))$ means $g(n)=O(f(n))$,

- $f(n)=\Theta(g(n))$ means $f(n)=O(g(n))$ and $g(n)=O(f(n))$, 
- $f(n)=o(g(n))$ means $\lim _{n \rightarrow \infty} \frac{g(n)}{f(n)}=\infty$,

- $f(n) \sim g(n)$ means $\lim _{n \rightarrow \infty} \frac{g(n)}{f(n)}=1$, and

- $f(n)=\omega(g(n))$ means $\lim _{n \rightarrow \infty} \frac{g(n)}{f(n)}=0$.

Example 1. Suppose $T_{A}(n)=\sum_{k=1}^{n} \frac{n}{k}, T_{B}(n)=2^{n}$, and $T_{C}(n)=\frac{1}{n+1}\left(\begin{array}{c}2 n \\ n\end{array}\right)$.

Then we have, as $n \rightarrow \infty$,

$$
\begin{aligned}
& T_{A}(n)=\Theta(n \log (n)), \\
& T_{B}(n)=\Theta\left(2^{n}\right), \\
& T_{C}(n)=\Theta\left(\frac{4^{n}}{n^{3 / 2}}\right) .
\end{aligned}
$$

By comparing $n \log (n), 2^{n}$, and $\frac{4^{n}}{n^{3 / 2}}$, we see $T_{A}(n)=o\left(T_{B}(n)\right)$ and $T_{B}(n)=$ $o\left(T_{C}(n)\right)$, as $n \rightarrow \infty$.

Asymptotics in this setting are perhaps surprisingly useful. The obvious objection to their use is that, for example, even though $\left(1+\exp \left(-10^{10}\right)\right)^{n}$ is in every asymptotic sense greater than $n^{100}$, the former is in every practical sense less than the latter. It is simply an observed fact that asymptotic statements are usually practically relevant, even though so much information is lost.

Thus, we see that the prototypical methodology in analysis of algorithms is the (incomputable in general) passage from algorithms to running time sequences to asymptotic expressions. (As always in mathematics, more abstract problems may be solved in analysis of algorithms which support this methodology indirectly.) This progression can be broken down into its two stages.

Obtaining exact running time sequences is closely related to the field of mathematics called enumerative combinatorics, a.k.a. counting. For example, let $S_{n}$ be the highest-cardinality set of states that an algorithm $A$ enters on an input of size $n$. Then $\left|S_{n}\right|$ is the worst-case running time of $A$ on inputs 
of size $n$. Counting sets such as $S_{n}$ is precisely the field of enumerative combinatorics. According to Wilf [39], when an algorithm is found that takes $n$ and returns $\left|S_{n}\right|$ and runs in at most exponential time, the combinatorial counting problem associated with the sets $S_{n}$ has been solved. To obtain these cardinality algorithms, a set of counting strategies/techniques is used, including the principle of inclusion-exclusion, recurrences, refinements, decompositions, and Pólya theory.

Instead of passing directly from a set to its cardinality, in enumerative combinatorics, establishing a bijection between two sets is a very common goal. If something has been proved about the cardinality of a set $S_{1}$, which is shown to be in bijection with $S_{2}$, the same result applies to $S_{2}$.

Problems in enumerative combinatorics may come from places other than analysis of algorithms, such as combinatorial number theory, algebra, and graph theory.

Given a sequence $\left(f_{n}\right)$, we define the ordinary generating function of $\left(f_{n}\right)$ to be

$$
F(z)=\sum_{n \geq 0} f_{n} z^{n}
$$

and we define the exponential generating function of $\left(f_{n}\right)$ to be

$$
\hat{F}(z)=\sum_{n \geq 0} f_{n} \frac{z^{n}}{n !}
$$

It turns out that these power series representations can be very useful for solving combinatorial problems, and especially useful for obtaining an asymptotic estimate of a sequence.

We define two common classes of power series. A power series $f(z) \in \mathbb{Q}[[z]]$ 
is algebraic iff there exist $p_{0}(z), \ldots, p_{j}(z) \in \mathbb{Q}[z]$ such that

$$
\sum_{k=0}^{j} p_{k}(z) f(z)^{k}=0
$$

A power series $f(z) \in \mathbb{Q}[[z]]$ is holonomic iff there exist $p_{0}(z), \ldots, p_{j}(z) \in \mathbb{Q}[z]$ such that

$$
\sum_{k=0}^{j} p_{k}(z) f(z)^{(k)}=0,
$$

where $f(z)^{(k)}$ is the $k$ th formal derivative of $f(z)$. Algebraic power series are holonomic [36].

\section{A few notes on Mathematica}

In order to fully understand all of the Mathematica code used in this thesis, the reader would have to be quite familiar with the Mathematica programming language. Ruskeepää's book [32] is ample preparation, for example. However, readers who are not familiar with Mathematica should still be able to follow the important ideas, and this section provides some essential Mathematica information to help in this regard.

The Mathematica code in this thesis is shown as it appears in an interactive session: There are input lines, which are typed in by the user after the prompt $\operatorname{In}[\mathrm{k}]:=$, and output lines, which Mathematica returns. Input lines that end in a semicolon do not produce an output line. Here is a basic example:

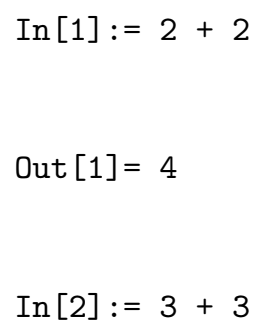


Out $[2]=6$

Every input line is numbered and its output line has the same number.

The character \% stands for the expression in the previous output line.

$\operatorname{In}[3]:=4-2$

Out $[3]=2$

$\operatorname{In}[4]:=\%-2$

Out $[4]=0$

In Mathematica, functions take their arguments in square brackets.

$\operatorname{In}[5]:=\operatorname{Sin}[\mathrm{Pi}]$

Out $[5]=0$

To write an anonymous function, write the value in terms of the argument \#, and place an \& at the end. In the next snippet, we define a function $f$ that squares its argument.

$\operatorname{In}[6]:=f=\#^{\wedge} 2 \quad \&$

$\operatorname{In}[7]:=f[4]$

Out $[7]=16$

Some of Mathematica's built-in functions use assumptions to control their behavior. They work like this: 


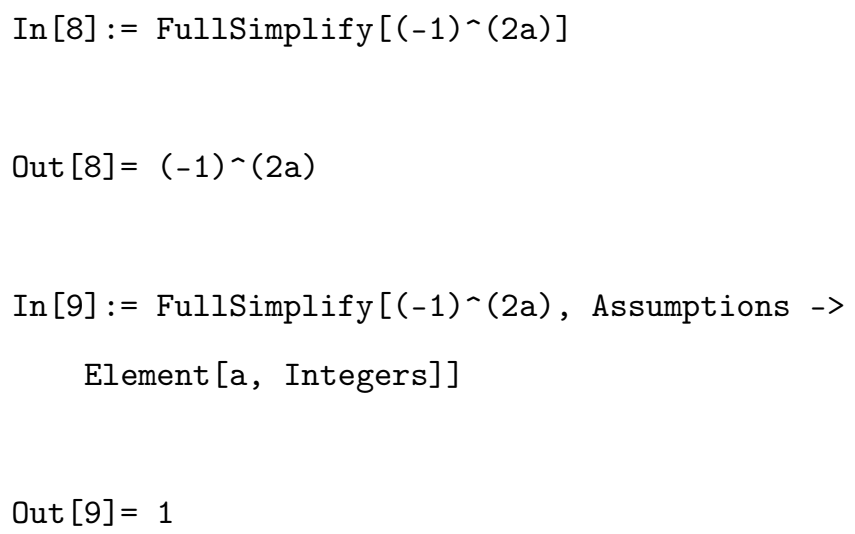

In In[9] we pass an Assumptions option to the algebraic simplifier function FullSimplify that says a can be assumed to be an integer. Alternatively, assumptions can be specified by setting the global \$Assumptions variable:

In $[10]:=$ \$Assumptions $=$ Element $[\mathrm{a}$, Integers $]$;

$\operatorname{In}[11]:=$ FullSimplify $\left[(-1)^{\sim}(2 a)\right]$

Out $[11]:=1$

Lists in Mathematica are made with braces and commas:

$\operatorname{In}[12]:=$ list $=\{5,4,3,2,1,0\}$;

$\operatorname{In}[13]:=$ Length [list]

Out $[13]=6$

Mathematica lists can contain anything:

$\operatorname{In}[14]:=1=\{5$, "this is a string", \{"string in a nested list!", True, symbol\}\};

It is perfectly fine for a function to not evaluate: 
$\operatorname{In}[15]:=\operatorname{Sin}[2]$

Out $[15]=\operatorname{Sin}[2]$

If we want the numerical value of $\sin (2)$, for example, we pass it to the $\mathrm{N}$ function:

$\operatorname{In}[16]:=N[\operatorname{Sin}[2]]$

Out $[16]=0.909297$

Mathematica has a huge number of built-in functions. To learn about a built-in function, simply search the online documentation [29].

\section{Guide to Genfunlib}

\subsection{Power series and their coefficients}

Converting between power series and their sequences of coefficients is a basic operation in computer algebra. In general, power series are represented by equations that they satisfy, which may be differential equations. This includes equations implied by "closed-form" expressions. Analogously, sequences may be given by recurrence relations or closed-form expressions.

It is tempting to say that when performing the conversion between power series and coefficient sequences, closed-form power series equations should become closed-form expressions for coefficients, and nontrivial power series equations should become recurrence relations. However, while it generally can, why should this aspect of the structure of an equation be preserved? Why not transform

$$
F(z)=\frac{1}{1-2 z}
$$


to

$$
f_{n}=2 f_{n-1}, \quad f_{0}=1 \quad \text { instead of } \quad f_{n}=2^{n} ?
$$

Indeed, if one wants a recurrence relation for computing initial terms, for computational efficiency reasons it is preferrable to have a recurrence relation that includes a finite set of lower-index terms, as opposed to one that uses the fullhistory of the sequence. Thus, unless the form that the result should be in is explicitly stated, there are many ways the translation can be performed [23].

Mathematica 9 includes some functionality for converting between power series and their coefficient sequences; Genfunlib extends this functionality by increasing the set of power series equations/recurrence relations on which the transformation can be applied.

Mathematica note: In computer algebra, representing a power series by an equation it satisfies is useful because of the level of generality it affords. Mathematica has support for two kinds of power series equation objects that can be used with Series and SeriesCoefficient.

Solutions to linear differential equations are represented as DifferentialRoot objects:

In $[17]:=$ FullSimplify [

SeriesCoefficient [

$\operatorname{DifferentialRoot}\left[\right.$ Function $\left[\{y, x\},\left\{y^{\prime}[x]==y[x], y[0]==1\right\}\right.$

]$[\mathrm{x}],\{\mathrm{x}, 0, \mathrm{n}\}]$

Assumptions :> Element $[\mathrm{n}$, Integers] \&\& $\mathrm{n}>=0]$

Out $[17]=1 / \mathrm{n} !$

Solutions to algebraic equations are represented as Root objects: 


\section{$\operatorname{In}[18]:=$ Series $\left[\operatorname{Root}\left[\#^{\sim} 5+a \#+1 \&, 1\right],\{a, 0,5\}\right]$}

\section{Out $[18]=-1+a / 5+a^{\wedge} 2 / 25+a^{\wedge} 3 / 125-\left(21 a^{\wedge} 5\right) / 15625+0[a] \sim 6$}

Genfunlib overrides the SeriesCoefficient command for extracting the coefficients of a power series.

Using Mathematica's built-in SeriesCoefficient we can compute the coefficients of explicit power series like $\exp (z)$.

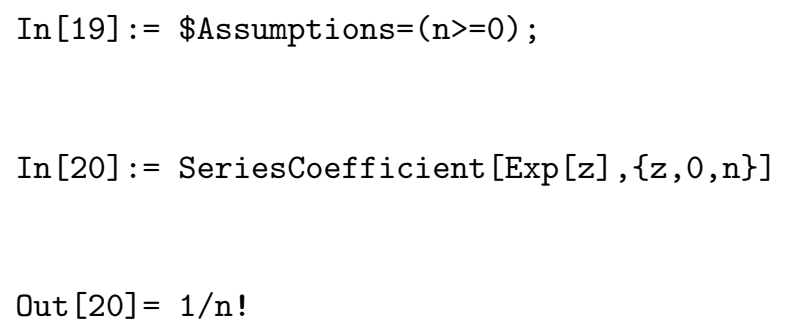

Genfunlib adds the ability to work with symbolic power series, as in the following example.

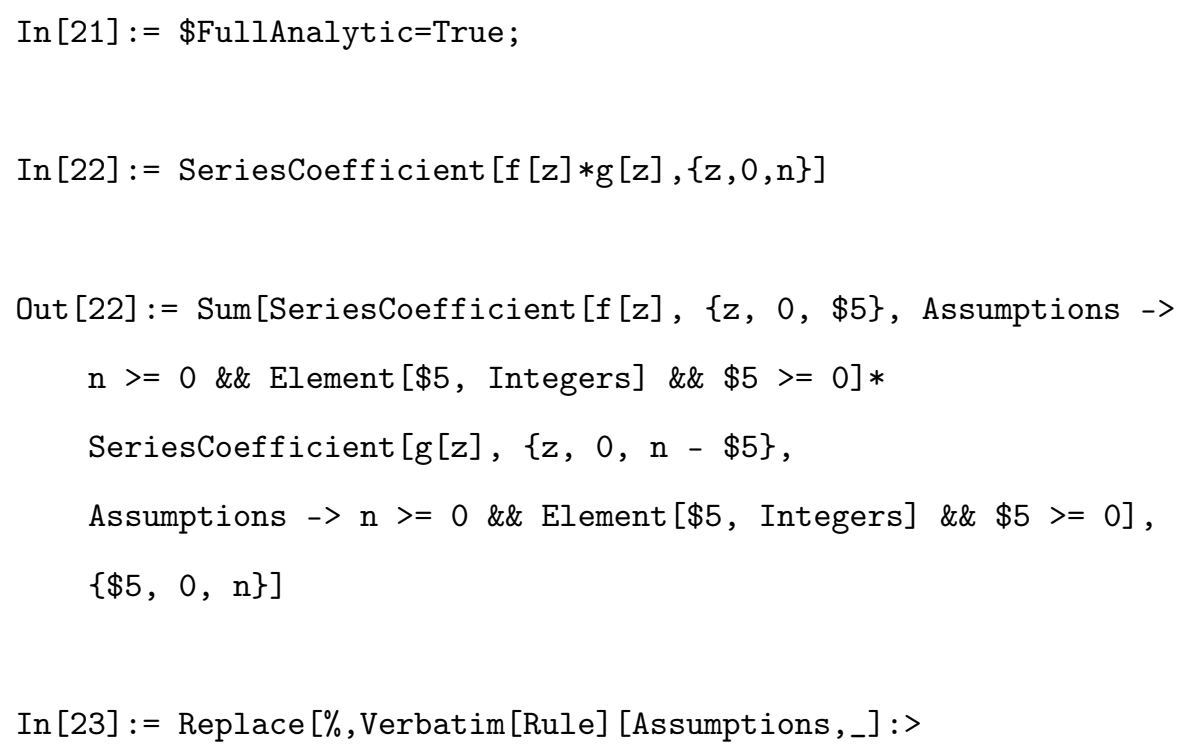


Sequence [], Infinity]

Out $[23]=$ Sum $[$ SeriesCoefficient $[f[z],\{z, 0, \$ 5\}] *$

SeriesCoefficient $[g[z],\{z, 0, n-\$ 5\}],\{\$ 5,0, n\}]$

In this snippet, we expand $\left[z^{n}\right](f(z) g(z))$. We first set the global option \$FullAnalytic=True. The \$FullAnalytic option, introduced in Genfunlib, determines whether or not expressions such as $f[z]$ should be assumed to be power series. In general, they could be Puiseux series

$$
\sum_{n \geq n_{0}} c_{n} z^{n / k}, \quad k \in \mathbb{Z}_{>0}, n_{0} \in \mathbb{Z}
$$

with negative and/or fractional exponents such as $\mathbf{z}^{\wedge}(-1 / 2)$. Assuming that we are working with power series (or analytic functions) allows us to unconditionally use the Cauchy product formula, for example.

\section{Mathematica note: The Mathematica command Series has the option} Analytic which is used for the same purpose as \$FullAnalytic.

Next, we extract coefficients from $f[z] * g[z]$ which returns a large expression containing Assumptions expressions. Using Replace to remove the assumptions, we get a result corresponding to $\sum_{j=0}^{n}\left(\left[z^{j}\right] f(z)\right)\left(\left[z^{n-j}\right] g(z)\right)$.

As well as products of symbolic power series, Genfunlib also adds support for sums, shifts, derivatives, integrals, and powers. The general form of the expansion of $\left[z^{n}\right] f(g(z))$ is an expression with multiple iterated sums that cannot be nicely represented in Mathematica notation, but special cases of power series composition such as $f(k z)$, for constant $k$, are handled by Genfunlib. Because Genfunlib enhances SeriesCoefficient with the ability to extract the coefficients of symbolic power series, we could use SeriesCoefficient on both sides 
of a power series equation and obtain a recurrence relation.

Mathematica's GeneratingFunction command is used for converting in the other direction: from coefficients to power series. In this case, the built-in version of GeneratingFunction comes with the ability to handle symbolic input.

In $[24]:=$ GeneratingFunction $[\operatorname{Sum}[f[j] * g[n-j],\{j, 0, n\}], n, z]$

Out $[24]=$ GeneratingFunction $[f[n], n, z]$ GeneratingFunction $[g[n], n, z]$

In this example, Mathematica's GeneratingFunction recognizes that the coefficient $\operatorname{Sum}[f[j] * g[n-j],\{j, 0, n\}]$ is in the form of a Cauchy product, and thus is the coefficient of the product of the generating functions of $f[n]$ and $\mathrm{g}[\mathrm{n}]$.

Genfunlib adds functionality to GeneratingFunction in a few areas. First, it handles coefficients of the form

$$
\frac{p(n)}{q(n)} f(n)
$$

where $p(n)$ and $q(n)$ are polynomials in the index symbol $n$, and $f(n)$ is symbolic. The generating function $\sum_{n \geq 0} \frac{p(n)}{q(n)} f(n) z^{n}$ can be expressed in terms of integrals and derivatives of $\sum_{n \geq 0} f(n) z^{n}$. Problems arise if $q(n)$ has positive integer roots, so we ignore that case. (Specifically, some coefficients would be undefined so it is not clear what the generating function should be.)

Mathematica note: Unfortunately, Mathematica 9 prevents the common case of $q(n)=1$ from working as expected. The GeneratingFunctions package of Mallinger [21] can be used for this, however. 
In $[25]:=$ GeneratingFunction $[\mathrm{f}[\mathrm{n}] /(\mathrm{n}+1), \mathrm{n}, \mathrm{z}]$

Out $[25]=$ Integrate $[$ GeneratingFunction $[f[n], n, \$ 3],\{\$ 3,0, z\}] / z$

This example shows that

$$
\sum_{n \geq 0} \frac{1}{n+1} f(n) z^{n}
$$

is automatically computed by Genfunlib to be

$$
z^{-1} \int_{0}^{z}\left(\sum_{n \geq 0} f(n) s^{n}\right) d s .
$$

Genfunlib also implements the "multi-section" formula for summing only on indices with a given residue modulo an integer.

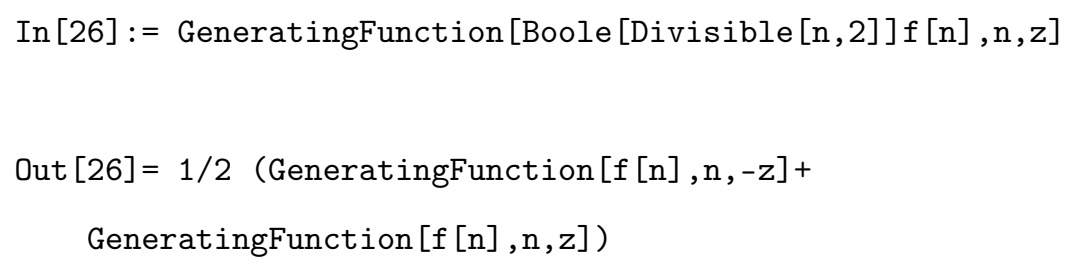

From the point of view of Genfunlib's source code, adding functionality to GeneratingFunction and SeriesCoefficient is straightforward using pattern matching and replacement rules. For example, the snippet

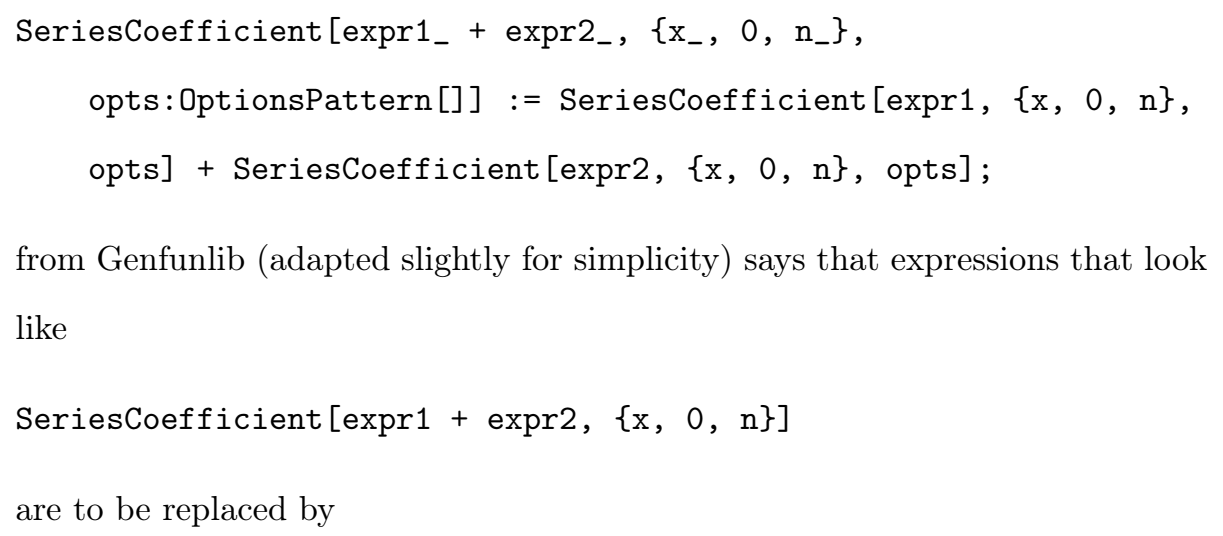


SeriesCoefficient $[\operatorname{expr} 1,\{x, 0, n\}]+$ SeriesCoefficient [

$\operatorname{expr} 2,\{\mathrm{x}, 0, \mathrm{n}\}]$

\subsection{Numerical coefficients}

Given a power series equation, it is a problem of computer algebra to evaluate the coefficients of the solutions. Obviously, computation speed is relevant, and for various sequence classes, there is active research around the problem of efficiently computing sequences in the class. $[2,3,5,17]$ One must note that there is a difference between computing all of the first $n$ terms of a sequence, and computing just the $n$ th. Computing $2^{n}$, for some integer $n$, is quickly done by using the method of repeated squaring. However, to compute $2^{0}, 2^{1}, \ldots, 2^{n}$, it is faster to simply perform one multiplication for each term. Genfunlib focuses on the problem of computing all initial terms.

\section{Mathematica note:}

One special type of power series equation is the inverse equation, which has the form

$$
z=\phi(f(z))
$$

where $f(z)$ is the unknown power series. Various algorithms can be used to compute the expansion of $f(z)$, including the Lagrange inversion formula and a more recent method due to Dominici [7]. Mathematica includes the function InverseSeries which can be used to solve this problem. In the following example the equation is $z=\sin (f(z))$.

$\operatorname{In}[27]:=\operatorname{Series}[\operatorname{Sin}[f],\{f, 0,10\}]$

Out [27] = f - f^3/6+f^5/120-f^7/5040+f^9/362880+o[f]^11 


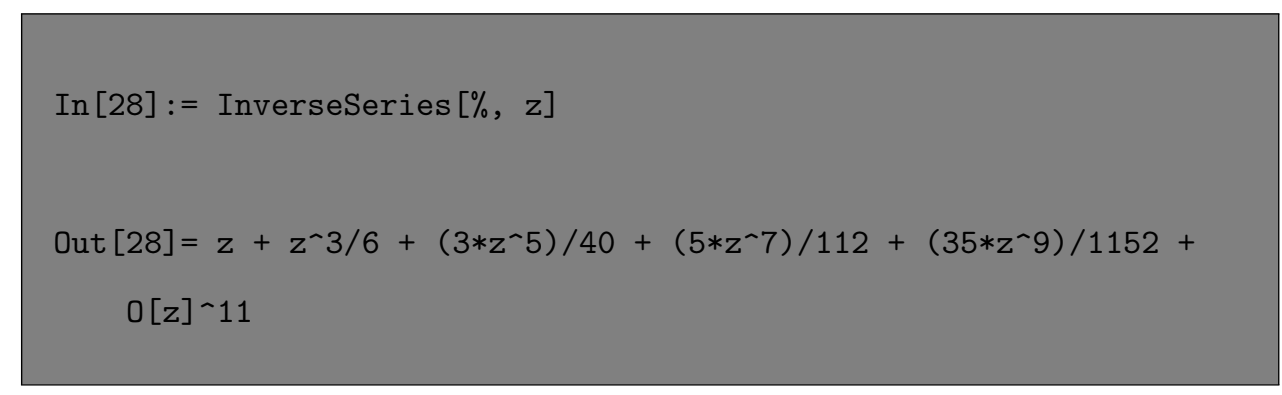

A very general method for computing coefficients of power series uses the Taylor coefficient formula

$$
\left[z^{n}\right] f(z)=\frac{f^{(n)}(0)}{n !}
$$

If an equation $F(f(z), z)=0$ is given in a power series $f(z)$, the value $\left[z^{0}\right] f(z)=$ $f(0)$ can be computed by setting $z=0$ and solving $F(f(0), 0)=0$ for $f(0)$. Then, for $n>0$, assuming we know $\left[z^{0}\right] f(z),\left[z^{1}\right] f(z), \ldots,\left[z^{n-1}\right] f(z)$, we compute and solve

$$
\left.\frac{d^{n}}{d z^{n}} F(f(z), z)\right|_{z=0}=0
$$

to find $\left[z^{n}\right] f(z)$. This can be generalized to differential equations, integral equations, etc.

Genfunlib implements this algorithm as the CoefsByDerivs command.

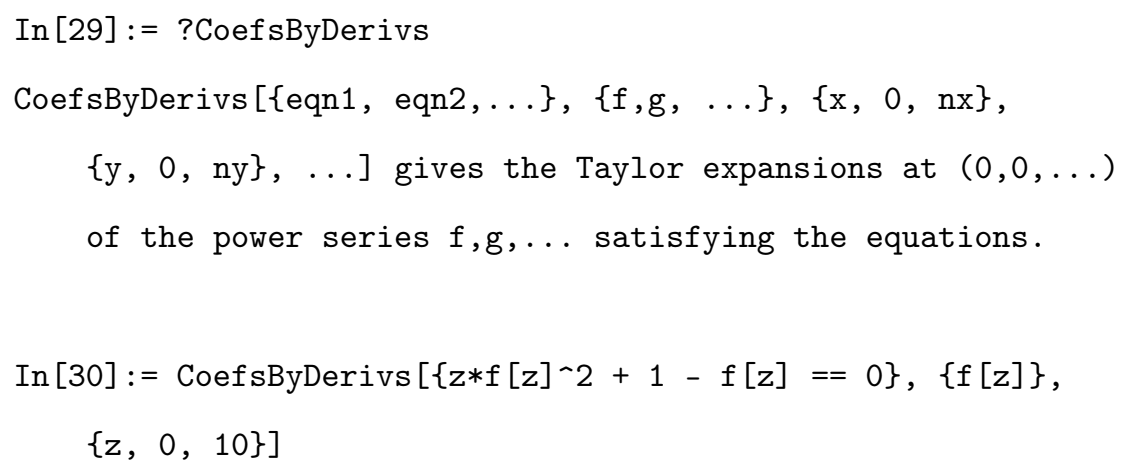




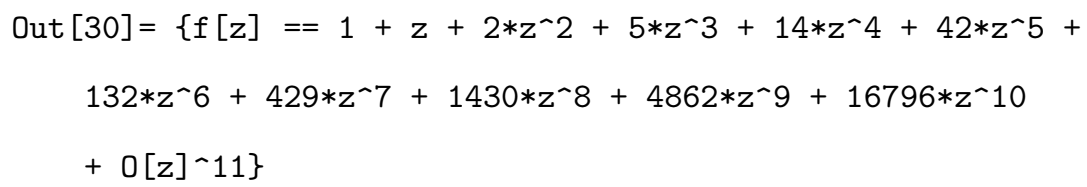

In this example, we pass a system of equations consisting of just one equation,

$$
z f(z)^{2}+1-f(z)=0
$$

then a list of power series which we want to expand, and a triple $\{z, 0,10\}$ indicating that the power series is in the indetermiate $\mathbf{z}$, the series is to be expanded around 0, and should have order 10. Genfunlib only supports expansions around 0 , but multivariate problems are possible.

\section{Mathematica note: Genfunlib calls Series to take the derivatives and} Solve to solve the equations.

The next example shows the expansion of $f(u, v)$ and $g(v)$ given by

$$
\begin{aligned}
f(u, v) & =u g(v) \\
g(2 v) & =\frac{1}{2} \exp (v),
\end{aligned}
$$

up to $\left[u^{4} v^{3}\right] f(u, v)$ and $\left[v^{3}\right] g(v)$.

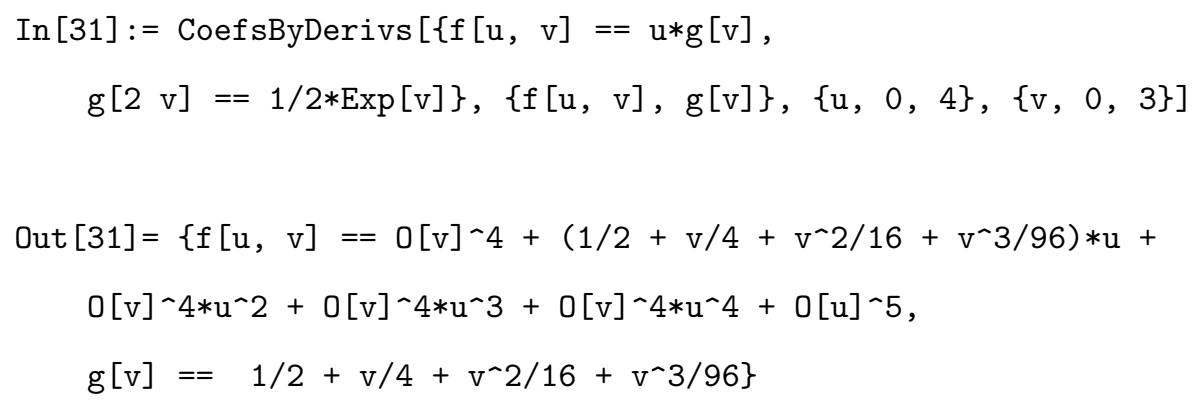


Newton iteration is a classical algorithm for numerically finding a zero of a continuously differentiable real function $f$. Starting at an arbitrary real number $x_{0}$, Newton iteration involves computing the sequence defined by

$$
x_{n+1}=x_{n}-\frac{f\left(x_{n}\right)}{f^{\prime}\left(x_{n}\right)} .
$$

Using a Taylor-Lagrange expansion of $f$ around $x^{*}$, we get

$$
0=f\left(x^{*}\right)=f\left(x_{n}\right)+f^{\prime}\left(x_{n}\right)\left(x^{*}-x_{n}\right)+\frac{1}{2} f^{\prime \prime}\left(\xi_{n}\right)\left(x^{*}-x_{n}\right)^{2},
$$

where $\xi_{n}$ is between $x_{n}$ and $x^{*}$. This equation implies

$$
x^{*}-x_{n+1}=\frac{-f^{\prime \prime}\left(\xi_{n}\right)}{2 f^{\prime}\left(x_{n}\right)}\left(x^{*}-x_{n}\right)^{2} \text {. }
$$

Thus, under some conditions on $f^{\prime}$ and $f^{\prime \prime}$, we have that the sequence $x_{n}$ in Newton iteration converges to $x^{*}$ quadratically.

If $f$ is not a real function but a mapping $\mathbb{Q}[[x]] \rightarrow \mathbb{Q}[[x]]$ defined by $y(x) \mapsto$ $F(x, y(x))$, where $F(x, y) \in \mathbb{Q}[[x, y]]$, Newton iteration can be applied to compute initial terms of the solution $y(x)^{*}$ of the power series equation

$$
F\left(x, y^{*}(x)\right)=0
$$

provided

$$
\left.\frac{\partial}{\partial y^{*}(x)} F\left(x, y^{*}(x)\right)\right|_{x=y^{*}(x)=0} \neq 0 .
$$

If we set $y_{0}(x)=\left[x^{0}\right] y^{*}(x)$, the key observation to make is that instead of Equation (1) referring to the distance between real numbers, we have

$$
y(x)^{*}-y_{n+1}(x)=c_{n}(x)\left(y(x)^{*}-y_{n}(x)\right)^{2},
$$


which implies that if $y(x)^{*}-y_{n}(x) \equiv 0\left(\bmod x^{2^{n}}\right)$, then $y(x)^{*}-y_{n+1}(x) \equiv 0$

$\left(\bmod x^{2^{n+1}}\right)$. In other words, the $n$th iteration step generates $2^{n}$ correct initial coefficients [38].

Genfunlib implements the Newton iteration method for computing expansions of solutions to power series equations with the CoefsByNewton command.

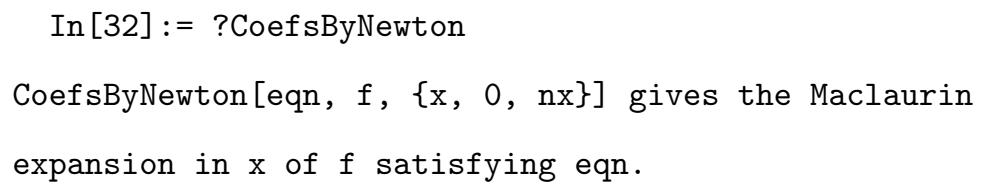

The code for CoefsByNewton comes partly from the implementation by Koepf [18] (which contains a slight bug on line 4 - compare with Genfunlib's source code for details). The expression

Coef sByNewton $[$ lhs $==r h s, f[x, y, \ldots],\{x, 0, n\}]$

where $\mathrm{lh} s==\mathrm{rhs}$ is an equation in $\mathrm{f}[\mathrm{x}, \mathrm{y}, \ldots]$, evaluates to the series expansion of $f[x, y, \ldots]$ around 0 in $x$ of order $n$. The following example shows the expansion of $f(z)$ given $z f(z)^{2}+1-f(z)=0$.

$\operatorname{In}[33]:=$ CoefsByNewton $[z * f[z] \sim 2+1-f[z]==0, f[z],\{z, 0,5\}]$

$\operatorname{Out}[33]=1+z+2 * z^{\wedge} 2+5 * z^{\wedge} 3+14 * z^{\wedge} 4+42 * z^{\wedge} 5+0[z] \wedge 6$

Genfunlib's CoefsByNewton cannot handle systems of equations, however if a system of equations can be broken up into independent equations by other methods, such as algebraic elimination [6], CoefsByNewton can be used.

\subsection{Regular languages}

A right regular grammar is a formal grammar $(N, \Sigma, P, S)$, where $N$ is a set of non-terminal symbols, $\Sigma$ is an alphabet, $P$ is a set of production rules, and 
$S \in N$ is the start symbol, such that all the production rules in $P$ are of one of the following forms:

- $B \rightarrow a$, where $B$ is a non-terminal symbol in $N$ and $a$ is a terminal symbol in $\Sigma$,

- $B \rightarrow a C$, where $B$ and $C$ are in $N$ and $a$ is in $\Sigma$,

- $B \rightarrow \epsilon$, where $B$ is in $N$ and $\epsilon$ is the empty string.

A word $w$ is generated by a right regular grammar if and only if, starting from $S$ we can obtain $w$ by applying a finite sequence of the production rules. A regular language is a set of words generated by a right regular grammar, or satisfying any of a number of alternative specifications. These include deterministic finite automata (DFA), nondeterministic finite automata (NFA), and regular expressions [35]. Any one of these specifications can be called a regular language representation (RLR).

Let $L$ be a regular language, let $|w|$ be the length of a word $w$ in $L$, and let

$$
c_{L}(n)=|\{w \in L:|w|=n\}| .
$$

In enumerative combinatorics, a key property of regular languages is that the ordinary generating function of $c_{L}(n)$, that is $\sum_{n \geq 0} c_{L}(n) z^{n}$, is a rational power series. This fact is hinted at by the linear structure of right regular grammars.

Indeed, this rational power series is computable in closed form, given an instance of an RLR. Let $r$ be a regular expression, and let $L(r)$ be the language it successfully parses. We say that $r$ is ambiguous iff there exists a word $w \in L(r)$ which can be parsed by $r$ in more than one way. Examples of ambiguous regular expressions include

$$
\epsilon^{*}, a^{* *}, b\left|b, a^{*}\right| b^{*}
$$


while examples of unambiguous regular expressions include

$$
a^{*}, a|a a| a a a, a|a b| \epsilon .
$$

It can be seen that if $r$ is an unambiguous regular expression, applying the following transformations to $r$ yields its generating function [11]:

- concatenation becomes multiplication,

- union becomes addition, and

- Kleene star becomes the quasi-inverse operation $1 /(1-\cdot)$.

Any RLR instance can be converted to a regular expression [35], so we can count the words in a regular language if we have an algorithm that takes a regular expression and returns an equivalent unambiguous one. It turns out that this can be done by simply converting from regular expression to NFA to DFA and back to regular expression according to the algorithms in [35]. Essentially, the "determinism" of a DFA carries over to a regular expression in the form of unambiguity. We note that Brabrand and Thomsen [4] give a useful algorithm for finding the particular subexpressions of a regular expression that gives rise to ambiguity.

Genfunlib implements a number of operations on RLR instances corresponding to some of their abundant decidable properties, and in particular, Genfunlib allows regular expressions to be disambiguated. Genfunlib allows regular languages to be represented by any of the following RLRs: NFA, DFA, regular expression, right regular grammar, or directed graph with labeled vertices. Directed graphs in which the vertices are labeled with letters are a nontraditional RLR which have the benefit that they may be visualized using Mathematica's built-in graph plotting. Such a graph $G=(V, E, l, \Sigma, S, E, e)$ is a set of vertices $V$, a set of edges $E$, a labeling function $l: V \rightarrow \Sigma$, where $\Sigma$ is an alphabet, a 
set $S \subseteq V$ of starting vertices, a set $E \subseteq V$ of ending vertices, and an integer $e$ which is 1 if $\epsilon$ is accepted and 0 otherwise. We say a labeled digraph $G$ accepts a nontrivial word $w$ if and only if there exists a path $v_{1}, v_{2}, \ldots, v_{k} \in V$ in $G$ with $v_{1} \in S$, and $v_{k} \in E$, such that $l\left(v_{1}\right) l\left(v_{2}\right) \cdots l\left(v_{k}\right)=w$.

Genfunlib allows an instance any RLR to be converted to an instance of any other. The following operations on RLRs are supported: union, intersection, complement, reverse, concatenation, and Kleene star. Generating functions for regular expressions can be computed by specifying a weight/marker for each letter in the alphabet.

Mathematica note: Since Mathematica does not use a type system, expressions must be inspected dynamically in order to determine whether they satisfy certain properties. The author of a Mathematica function must decide how to handle input expressions that the function is not supposed to take. One example of this is a function on the integers being passed a symbol rather than an integer - there are many reasons why a user might want to do this. According to the Guidebook for programming [37],

As a rule of thumb, messages are not generated for "symbolic" input if the function they appear in is used in classical mathematics.

Thus Table $[1,\{n\}]$ generates a message, while Sin $[z]$ does not. Genfunlib attempts to follow this convention, so all functions related to regular languages do not accept symbolic input or input that cannot be directly used for computation.

In Mathematica, input validation checks can be complete or shallow. A shallow check would only make sure the input is in roughly the right form, but would not check whether the input would cause an error later on. Generally, openly available Mathematica packages do not perform as much validation as 
functions in the Mathematica kernel.

The FiniteFields package largely does not do input validation. It sometimes performs shallow syntactic validity checks, sometimes performs total semantic validity checks, and sometimes sends error messages. (On failure, all checks result in an expression returning unevaluated). The Splines package does only shallow syntactic input validation. Mathematica built-in functions often validate any sequence of arguments and send messages on errors, and generally do not produce results that will cause errors "later on".

Since Mathematica expressions must be inspected dynamically, one may seek a method of caching properties of expressions explicitly to avoid overcomputation. For example, a function may always return a prime number, so the values of this function do not need to be tested later on for primality. In Genfunlib, the result of a successful RegUnion command, for example, is guaranteed to be a valid RLR, but when it is passed to another function, it is checked for validity anyway. One possible strategy is for all functions to store the validity of their results right before they return them, by setting a value of the "validate" function, which would look something like this:

validate $[$ ret $]=$ True; Return $[$ ret $]$

This system could be altered by making the validate symbol only remember the last $n$ such expressions to save memory.

If a public function calls another public function, it presumably always passes valid input. One way to avoid unnecessary computation of the validity is to pass an option saying "validation not required"; another is for public functions never to call public functions.

The validation scheme of Genfunlib is as follows. Public functions call validation directly (right in their definition) unless told not to by the 
validationRequired option; private functions do not do validation. Using validationRequired saves some computation at the expense of more complicated code. Data representation symbols like DFA do not do validation themselves; indeed they are not defined as functions. This follows the convention exhibited in Mathematica symbols like RegularExpression and Graph.

The authors of the Combinatorica package for Mathematica [25] say, "Our aim in introducting permutation groups into Combinatorica is primarily for solving combinatorial enumeration problems. We make no attempt to efficiently represent permutation groups or to solve many of the standard computational problems in group theory." The situation for this package and automata/grammar algorithms is similar - performance is not the highest priority.

In Genfunlib, letters are represented by nonempty Strings and words are represented by Lists of letters.

Mathematica note: An alternative approach to providing regular language functionality in Mathematica would have been to make a J/Link interface to the dk.brics.automaton Java package available at http://www.brics. $\mathrm{dk} /$ automaton/.

The expression formats used by Genfunlib for each RLR are as follows.

An NFA expression is of the form

NFA [numStates, alphabet, transitionMatrix, acceptStates, initialState]

The number of states is a nonnegative integer; any NFA with zero states accepts no words. The alphabet is a sorted list of distinct strings, not containing " ". A value of \{\} (the empty list) means the NFA accepts either the empty language 
or $\{\epsilon\}$. The transition matrix is a numStates by Length [alphabet]+1 matrix where entry $i, j$ is a list of integers corresponding to states accessible from state $i$ and, if $j<=$ Length [alphabet], on the letter alphabet [ $[j]]$, otherwise, on an $\epsilon-$ move. If the number of states is zero, then we must have transitionMatrix $==\{\}$. If the alphabet is empty, then transitionMatrix has one column (if it has any rows). The accept states are a list of integers between 1 and the number of states. The initial state is an integer between 1 and number of states, or Null iff numStates $==0$.

As an example, we can represent the NFA in Figure 1 by the following expression.

NFA $[6,\{" 0 ", " 1 "\},\{$

$\{\{2\},\{2\},\{3\}\}$,

$\{\{4\},\{2\},\{\}\}$,

$\{\{4\},\{2\},\{\}\}$,

$\{\{6\},\{3\},\{\}\}$,

$\{\{\},\{\},\{\}\}$,

$\{\{4\},\{5\},\{\}\}$

\}$,\{3,4\}, 1]$

A regular expression that is equivalent to the NFA in Figure 1 is

$$
\begin{aligned}
& \left(( ) | 1 ( 1 ) ^ { * } 0 | 1 ( 1 ) ^ { * } 0 0 ( 0 0 ) ^ { * } 0 | 0 ( 0 ) ^ { * } | \left(1(1)^{*} 01 \mid 1(1)^{*} 00(00)\right.\right. \\
& \left.{ }^{*} 01 \mid 0(0)^{*} 1\right)\left(\left(\left(0 \mid 1(1)^{*} 0\right) 1 \mid\left(0 \mid 1(1)^{*} 0\right) 0(00)^{*} 01\right)\right)^{*}(() \mid 0 \\
& \left.\left.\left|1(1)^{*} 0\right|\left(0 \mid 1(1)^{*} 0\right) 0(00)^{*} 0\right)\right) .
\end{aligned}
$$

A DFA expression is of the form

DFA[numStates, alphabet, transitionMatrix, acceptStates, initialState] 


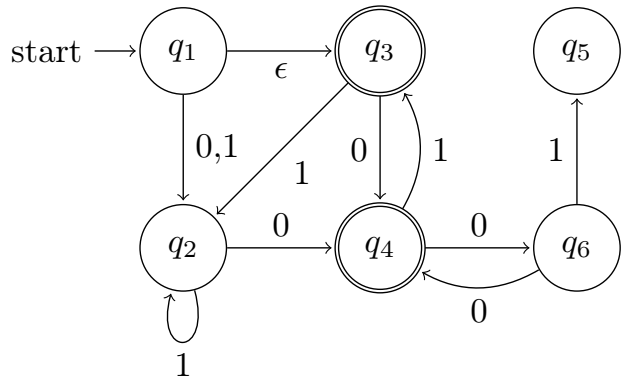

Figure 1: A six-state NFA.

The number of states is a nonnegative integer; any DFA with zero states accepts no words. The alphabet is a sorted list of distinct strings, not containing " ". A value of \{\} means the DFA accepts either the empty language or $\{\epsilon\}$. The transition matrix is a numStates by Length [alphabet] matrix where entry $i, j$ is the state accessible from state $i$ and letter alphabet [ $[j]]$. If the number of states is zero, then transitionMatrix $==\{\}$. If the alphabet is empty, then transitionMatrix $==\{\{\},\{\}, \ldots\}$. The accept states are a list of integers between 1 and the number of states. The initial state is an integer between 1 and the number of states, or Null if numStates $==0$.

As an example, we can represent the DFA shown in Figure 2 with the following expression.

DFA $[5,\{" 0 ", " 1 "\},\{$

$\{2,3\}$,

$\{2,4\}$,

$\{4,3\}$,

$\{5,5\}$,

$\{5,5\}$

\}$,\{4\}, 1]$ 


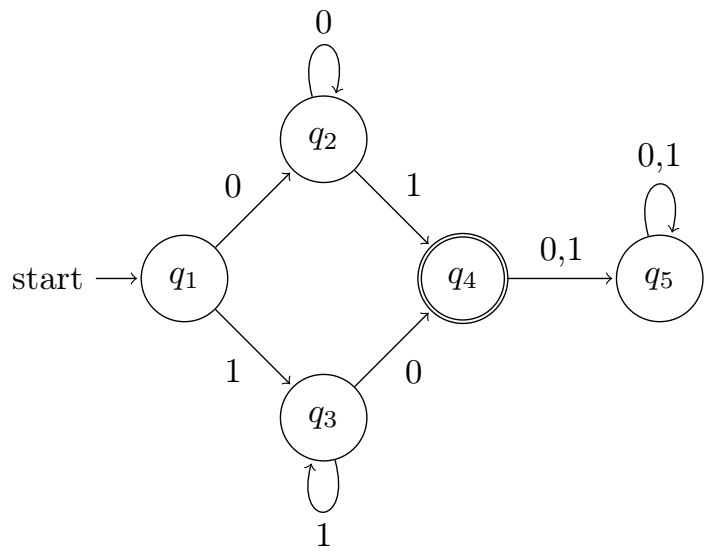

Figure 2: A five-state DFA.

A regular expression that is equivalent to the DFA in Figure 2 is

$$
\left(0(0)^{*} 1 \mid 1(1)^{*} 0\right) .
$$

In Genfunlib, a regular expression is represented by an expression with head Regex, and an argument built up from nonempty strings, EmptyWord, RegexStar, RegexConcat, and RegexOr. The expression Regex [Null] matches no words (the empty language).

As an example, the regular expression $(a \mid b b)^{*} b b b$ is represented as Regex [RegexConcat [RegexStar [RegexOr ["a", RegexConcat ["b", "b"]]], "b", "b", "b"]]

Applying Mathematica's TreeForm command to this expression produces Figure 3 .

Right regular grammars are represented in Genfunlib by expressions with head RRGrammar, and one argument which is a list of rules in the form sym -> RHS or sym[n] -> RHS, where sym is a symbol, $\mathrm{n}$ is an integer, and RHS is either - EmptyWord, 


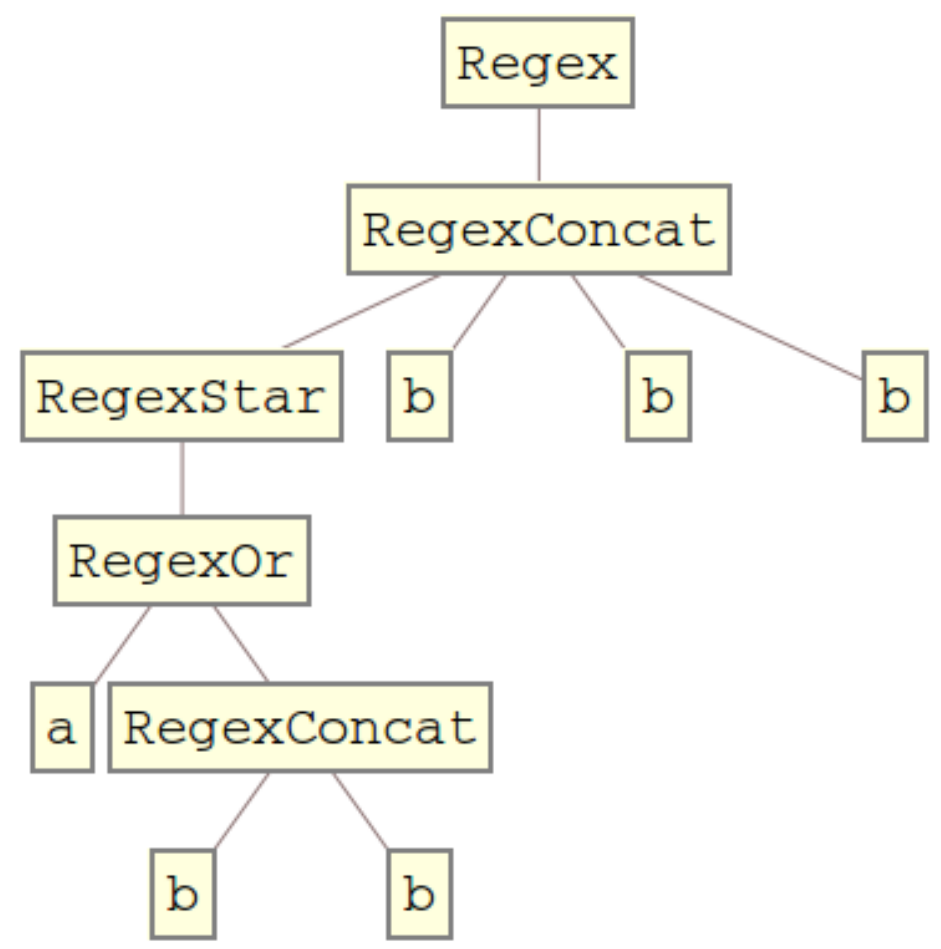

Figure 3: The parse tree of $(a \mid b b)^{*} b b b$. 
- a string,

- an expression on the left hand side of another rule,

- RRGrammarConcat [str, sym] (where str is a string),

- RRGrammarConcat [str, sym[n]], or

- RRGrammarOr [args], where args is a sequence of any of the things in this list.

Strings cannot be empty. An empty list corresponds to the empty language.

For example, let $G$ be the right regular grammar such that $N=\{S, A\}$, $\Sigma=\{a, b, c\}$, and the production rules $P$ are

$$
\begin{aligned}
& S \rightarrow a S \\
& S \rightarrow b A \\
& A \rightarrow \epsilon \\
& A \rightarrow c A .
\end{aligned}
$$

It can be seen that $G$ is equivalent to the regular expression $a^{*} b c^{*}$. In Genfunlib, $G$ becomes

RRGrammar $[\{$

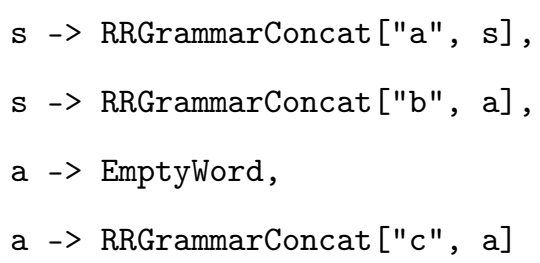

A directed graph with labeled vertices is represented by an expression of the form Digraph[graph, startVertices, endVertices, eAccepted]. The 
graph is a directed Mathematica Graph, whose vertices are all labeled with nonempty strings. The starting vertices are a list of vertices of the graph; if this list is empty, the language associated with the graph contains no words except possibly $\epsilon$. The ending vertices are a list of vertices of the graph; if this list is empty, the language associated with the graph contains no words except possibly $\epsilon$. Finally, eAccepted is True if $\epsilon$ is accepted and False otherwise.

Any of the five RLRs can be converted to any other using the commands ToNFA, ToDFA, ToRegex, ToRRGrammar, and ToDigraph.

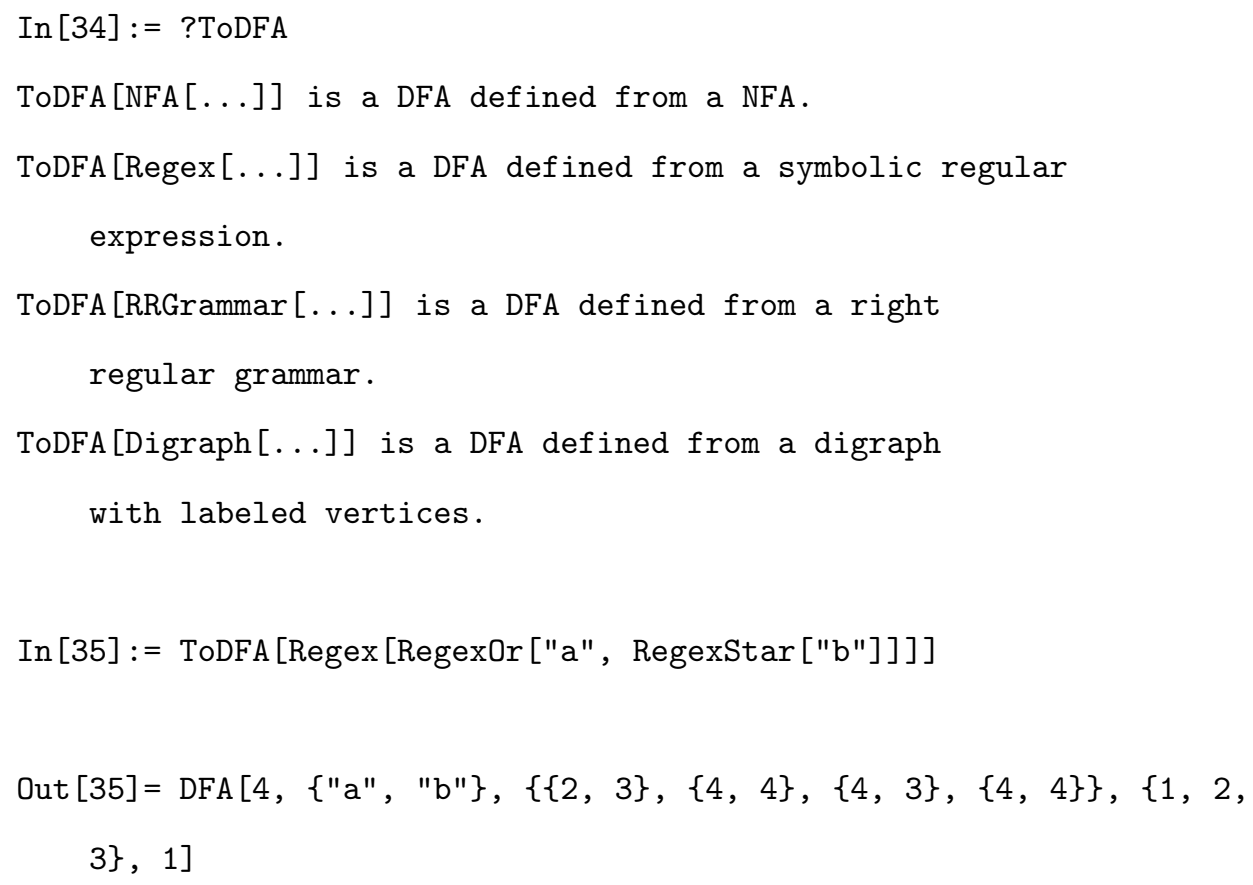

The algorithms for performing these conversions come from the textbook of Sipser [35] with two exceptions. First, given an NFA $M$ with state set $Q$, the standard algorithm for obtaining a DFA that is equivalent to $M$ involves computing the powerset $2^{Q}$, which is used as the state set for the DFA. In some cases, there is no DFA equivalent to $M$ with a state set with fewer elements than 
$2^{Q}[34]$. However, the algorithm used in Genfunlib produces an automaton with many fewer states, when this is possible. The first step is to remove states from the NFA that are inaccessible from the start state or from every accepting state. Each state removed halves the maximum size of the resulting DFA. Next, when computing the set of states for the DFA, Genfunlib only includes those sets in $2^{Q}$ that are accessible from the initial state by performing an incremental search starting at the initial state. Finally, the DFA is minimized using Hopcroft's algorithm [35]. Experimental tests showed a huge speed increase when using these steps to avoid creating a DFA with $2^{|Q|}$ states. In testing, Genfunlib found the minimal DFA of an NFA with 379 states in under half an hour on a computer with an AMD Phenom II X4 995 CPU and 8 gigabytes of RAM. However, the resulting DFA had only 8 states, so in terms of the length of the output, Genfunlib's performance still has room for improvement. The dk.brics.automaton Java package [22] uses a similar determinization algorithm, but the performance of that library is much higher than that of Genfunlib, presumably because Java is a lower-level language than Mathematica.

The other somewhat unusual conversion algorithm used in Genfunlib is the one that maps labeled digraphs to automata and vice versa, since the digraph RLR is not included in textbooks on automata theory. Because in Genfunlib's digraphs, vertices are labeled, and in the graphs derived from automata, edges are labeled, something like the line digraph construction can be used. The line digraph of a digraph $G$ has one vertex for each edge of $G$, and has an edge between two of its vertices if and only if the corresponding edges in $G$ form a length-two directed path in G. "Graphs" derived from automata are actually hypergraphs, but the line digraph definition can be adapted to this case. When Genfunlib converts something to a DFA, the output is a minimal DFA, but when converting to labeled digraphs or NFAs, the output may not be minimal. 


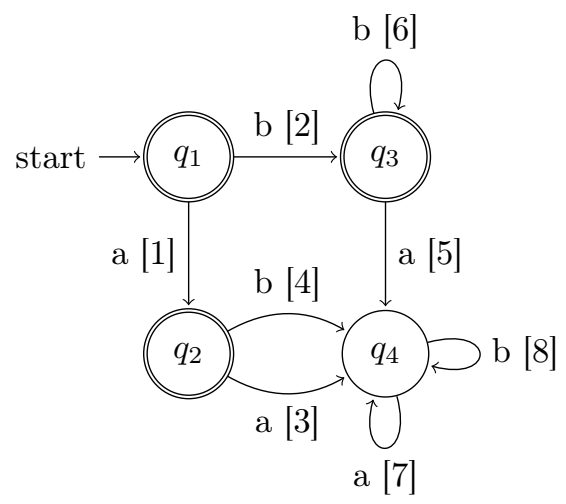

Figure 4: A DFA with eight transitions.



Figure 5: The digraph constructed from the DFA in Figure 4. Vertices are labeled by the transition they correspond to in the DFA.

Minimizing NFAs is a PSPACE-complete problem [14].

As an illustration, the DFA in Figure 4 has each of its "edges" shown with an integer label, in brackets. Genfunlib converts this DFA to the digraph shown in Figure 5. The vertices of this digraph are shown with extra labels in brackets indicating the edge in the original DFA to which they correspond.

In addition to conversions between the five RLRs, Genfunlib includes functions for converting between Genfunlib regular expressions and Mathematica built-in regular expressions, which have head RegularExpression. To be converted, the Mathematica regular expressions must have a restricted syntax: 
Only letter characters, parentheses, asterisks, and bars (|) are allowed.

The following example converts the regular expression $(c \mid a)^{*}$ from Mathematica syntax to Genfunlib syntax and back, and demonstrates how built-in Mathematica functions such as StringMatchQ can be used with Mathematica regular expressions.

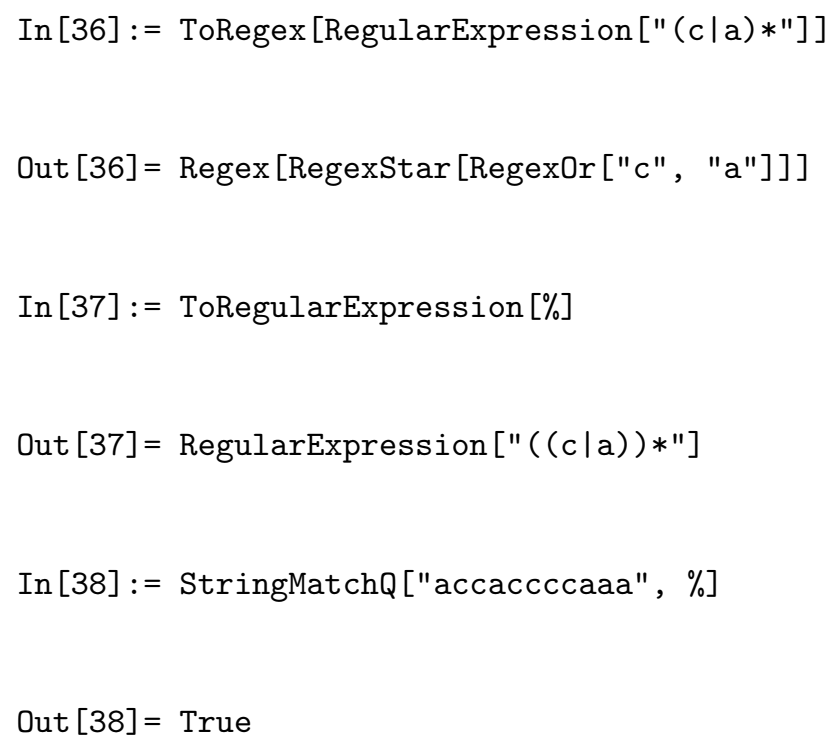

Since, in Genfunlib, letters are represented by strings which may be longer than one character (and words are represented by lists of strings), some issues may arise when converting to Mathematica regular expressions. In Genfunlib, \{"a", "aa"\} and \{"aa", "a"\} are different words. However, since in Mathematica represents letters as length-one strings and represented words as strings,

Regex [RegexOr[RegexConcat ["a", "aa"], RegexConcat ["aa", "a"]]]

gets converted to

RegularExpression [" (aaa|aaa)"]

Regular languages have a rich set of closure properties [35], which Genfunlib implements in the form of six operations: Kleene star (RegStar), comple- 
ment (RegComplement), reverse (RegReverse), union (RegUnion), concatenation (RegConcat), and intersection (RegIntersection).

The algorithms for performing these operations all come from Sipser's textbook [35]. For each operation, Genfunlib implements such an algorithm that works for one type of RLR. Then the operation is implemented for all other RLR types by converting them to the type for which an algorithm is implemented, running the algorithm, then converting back again. As an example, the concatenation operation is directly implemented only for right regular grammars, but users can call RegConcat on two DFAs because they will get automatically converted to and from grammars.

The unary operations RegStar and RegReverse simply take one RLR as an argument. The operation RegComplement takes one RLR $r$ and an alphabet $\Sigma$ and returns an RLR $r^{\prime}$ of the same type as $r$ such that $L\left(r^{\prime}\right)=\Sigma^{*} \backslash L(r)$, where $L(r)$ is the language of $r$.

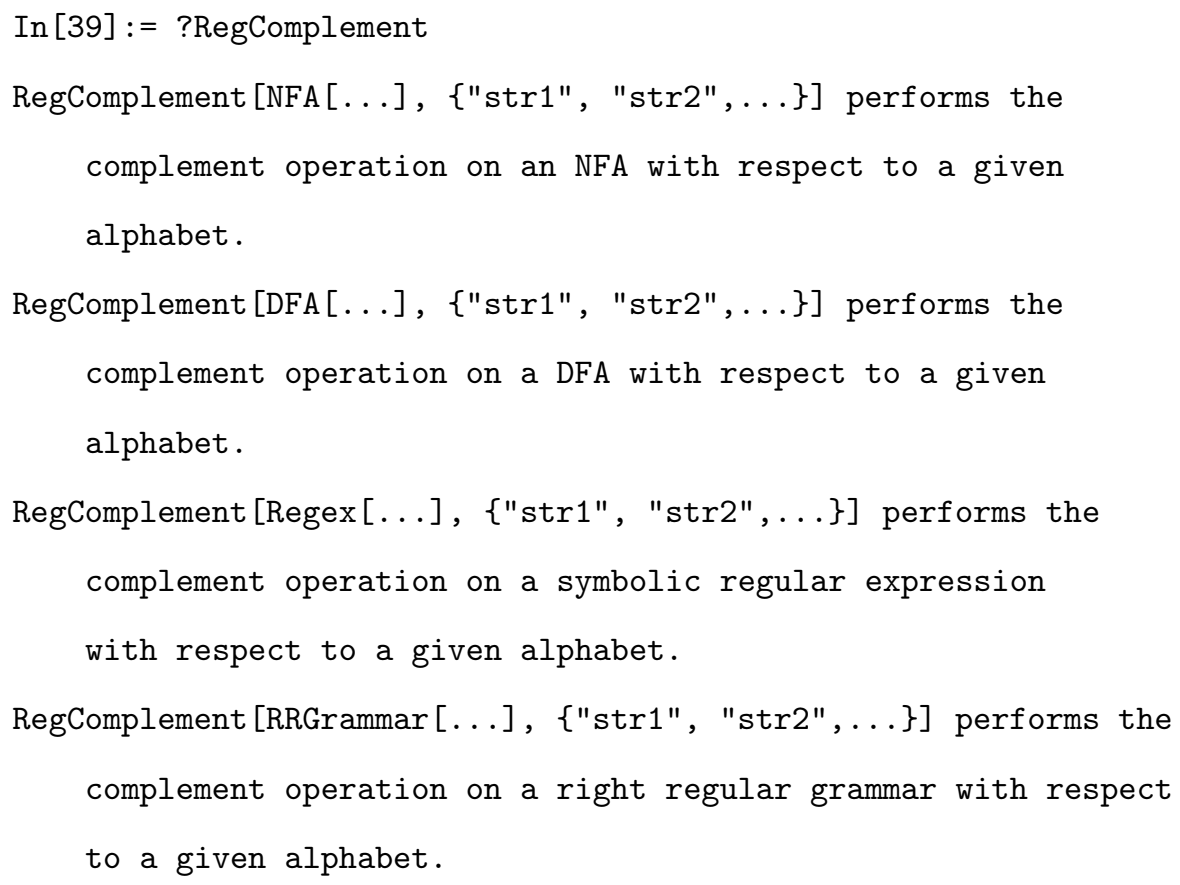




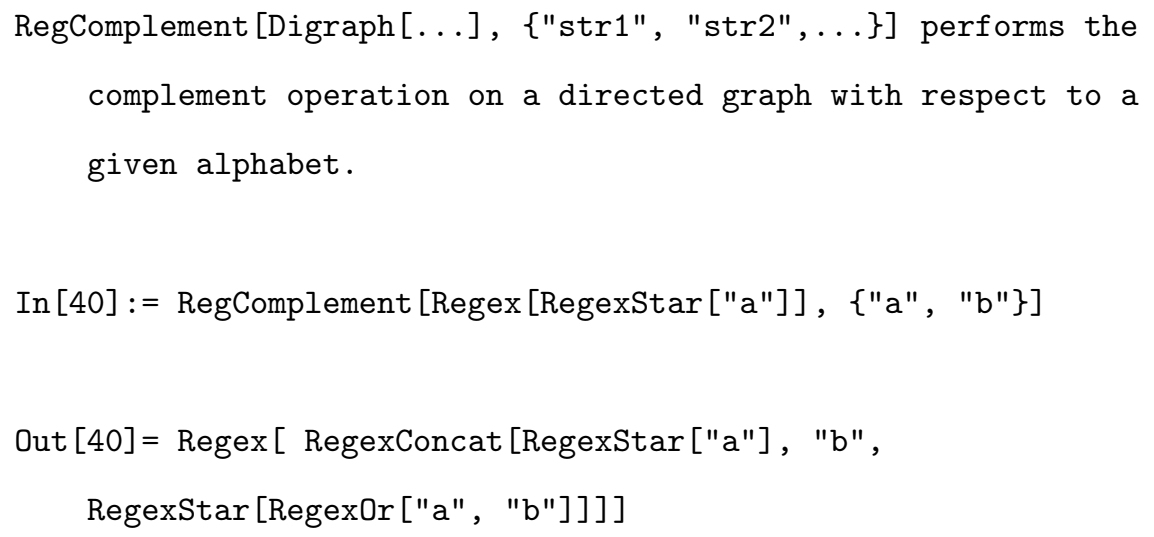

The binary operations RegUnion, RegConcat, and RegIntersection take two RLRs of the same type.

Genfunlib overloads GeneratingFunction to take a regular expression and a list of rules mapping letters to weights. The regular expression is automatically disambiguated, and the letters are replaced with their weights to obtain a rational power series. Here we compute the generating function for the ambiguous regular expression $a|a| b \mid b^{*}$ that marks $a$ with $u$ and $b$ with $v$ :

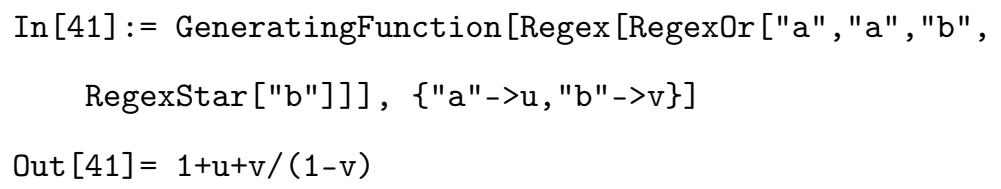

\subsection{Symbolic method}

Genfunlib contains a basic implementation of the symbolic method. An appropriate introduction to the symbolic method may be found in the author's honours project [20] or Flajolet and Sedgewick's book [11].

Genfunlib allows combinatorial classes to be defined by specifications, and can convert these specifications to corresponding systems of equations in generating functions.

A specification is represented by an expression of the form 


\begin{tabular}{|l|l|l|l|}
\hline \multicolumn{2}{|c|}{ Labeled } & \multicolumn{2}{c|}{ Unlabeled } \\
\hline Sum & SMPlus & Sum & SMPlus \\
\hline Product & SMTimes & Product & SMTimes \\
\hline Sequence & SMSeq & Sequence & SMSeq \\
\hline Cycle & SMCyc & Cycle & SMCyc \\
\hline Set & SMSet & Powerset & SMSet \\
\hline Pointing & SMPointing & Multiset & SMMultiset \\
\hline Substitution & SMSub & Pointing & SMPointing \\
\hline & & Substitution & SMSub \\
\hline
\end{tabular}

Table 1: Admissible constructions supported by Genfunlib.

$\operatorname{Spec}[\{$ lhs $1==r h s 1, \ldots\}$, labeled? $]$

In the list of equations, the left hand sides are symbols representing classes, and the right hand sides are expressions built with combinatorial constructions, symbols found in a left hand side, restrictions (Restricted $[\ldots]$ ), and atomic and neutral classes. The second argument is True if the classes are labeled, and False if they are unlabeled.

Genfunlib supports multiple atomic classes, each of which is indexed by a positive integer and is marked seperately in generating functions. They have the form ZClass[1], ZClass [2],... The neutral class is represented by the symbol EClass.

The combinatorial constructions in Genfunlib are shown in Table 1, along with their syntax. Sum and product are commutative binary constructions; sequence, cycle, set, multiset, and pointing are unary constructions. Substitution is a binary construction, but it is not commutative. The expression SMSub [A, B] corresponds to $\mathcal{A}(\mathcal{B})$, if $\mathcal{A}$ is represented by the symbol $\mathrm{A}$ and $\mathcal{B}$ is represented by the symbol B.

The set, multiset, sequence, and cycle constructions take an optional second argument in the form Cardinality->f, where $f$ is a predicate on the integers which gives the allowed number of components. For example, 
SMSeq[class, Cardinality $->(\#<=k \&)]$

represents the class of finite sequences of elements of class with at most $k$ terms. It is fine to use a symbol like $\mathrm{k}$ rather than an integer.

The other kind of restriction that can be done is with the Restricted symbol. The expression Restricted [class,f], where $f$ is a predicate on the integers, represents the class of objects from class with sizes that $f$ maps to True. For example, Restricted [SMSeq[SMTimes[ZClass[1],ZClass[2]]], \#>2\&] represents the class containing all the objects in $\operatorname{SEQ}\left(\mathcal{Z}_{1} \times \mathcal{Z}_{2}\right)$ except the singleton sequence.

\section{Mathematica note: Predicates on the integers must return unevaluated} when given symbolic input. The predicate $\operatorname{Mod}[\#, 2] \&$ satisfies this requirement, while PrimeQ[\#]\& does not.

The command for passing from a combinatorial specification to a system of generating function equations is ToGFEqns. It takes two arguments, the first is a Spec expression and the second is a symbol to be used as the indeterminate. If the symbol $\mathrm{x}$ is supplied, ZClass [1] is marked with $\mathrm{x}$ [1], ZClass [2] is marked with $\mathrm{x}[2]$, and so on. In the output, the generating function of a class is written as the symbol for the class, with arguments.

$\operatorname{In}[42]:=$ ?ToGFEqns

ToGFEqns [spec, indeterminate] gives the system of power series equations corresponding to spec, using the given indeterminate.

$\operatorname{In}[43]:=$ ToGFEqns [ 
$\operatorname{Spec}[\{c==\operatorname{SMTimes}[\mathrm{ZClass}[1], \operatorname{SMSeq}[\mathrm{ZClass}[1]]]\}, \operatorname{True}], \mathrm{z}]$

$\operatorname{Out}[43]=\{c[z[1]]==z[1] /(1-z[1])\}$

The code that performs the translation to generating functions uses pattern matching and replacement rules. The following example snippet is a simplified segment of Genfunlib's code that takes a specification called spec and replaces, e.g. ZClass [2] and ZClass [3] with z [2] and z [3], and replaces EClass with 1.

Replace [spec,

\{ ZClass $\left[n_{-}\right]:>z[n]$, EClass :> 1,

\} ,

$\{1$, Infinity $\}$, Heads $->$ True

];

Genfunlib includes one more command related to the symbolic method: ToGenfunlibSpec. This command takes two arguments. The first is a string containing the Maple code for a Combstruct grammar in the format used on the Encyclopedia of Combinatorial Structures (ECS) website at

http://algo.inria.fr/ecs/

The second argument is a Boolean value indicating whether the classes are labeled. The output is a Spec expression representing the same specification as the Combstruct grammar. Since Genfunlib and Combstruct specifications are similar in structure, the conversion is not complex and mostly consists of termfor-term translation. Class symbols are converted from upper case to lower case (and reduplicated) to conform to Mathematica conventions. 
As an example, we can take entry $\# 9$ from the ECS: words on the alphabet $\{a, b\}$ with at most one consecutive $b$-letters. The ECS gives the following unlabeled Combstruct specification:



In Genfunlib, the class $\mathrm{S}$ becomes ss.

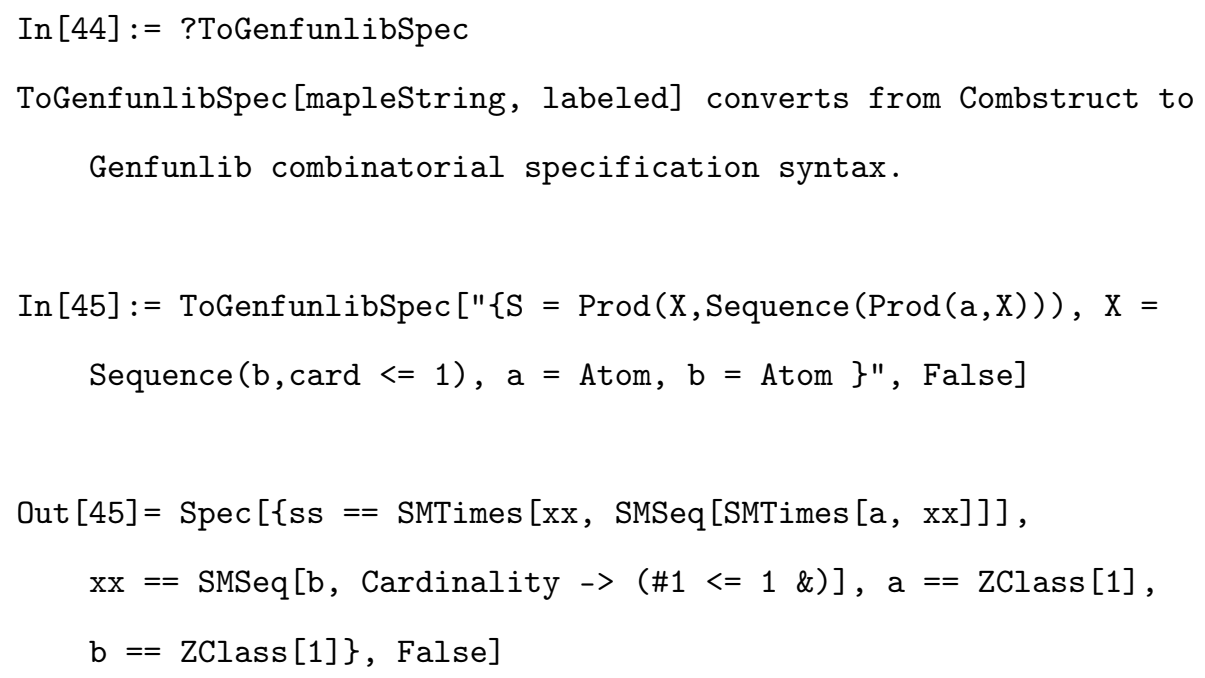

We can convert to generating functions using indeterminate $\mathbf{z}$ and solve for $\operatorname{ss}[z[1]]$.

In $[46]:=$ ToGFEqns $[\%, z]$

Out $[46]=\{\operatorname{ss}[z[1]]==\operatorname{xx}[z[1]] /(1-\mathrm{a}[z[1]] \mathrm{xx}[z[1]])$, $\mathrm{xx}[\mathrm{z}[1]]==1+\mathrm{b}[\mathrm{z}[1]], \mathrm{a}[\mathrm{z}[1]]==\mathrm{z}[1], \mathrm{b}[\mathrm{z}[1]]==\mathrm{z}[1]\}$

$\operatorname{In}[47]:=$ Solve $[$ Eliminate $[\%,\{a[z[1]], b[z[1]], x x[z[1]]\}]$, $\operatorname{ss}[z[1]]]$ 
$\operatorname{Out}[47]=\{\{\operatorname{ss}[z[1]]->(-1-z[1]) /(-1+z[1]+z[1]-2)\}\}$

There is room for expansion of Genfunlib's symbolic method capabilities. Symbolic method specifications are useful because tractable operations can be performed on them. This is of note since in general, combinatorial objects are too complex for such simple notation. Assuming the user is able to provide a combinatorial specification, one valuable question may be whether the specification is well-formed. The class $\mathcal{I}$ defined by $\mathcal{I}=\operatorname{SEQ}(\mathcal{E})$ has an infinite set of objects of size 0 , so the specification should be edited before anything is done with it. Work on checking specifications for well-definedness has been published by Salvy and coauthors [10,26]. Genfunlib does not implement these complete validity checks on specifications; however, errors are likely to be found at a later state when working with power series. For example, $\mathcal{I}=\operatorname{SEQ}(\mathcal{E})$ would generate a division by 0 error.

Lastly, there exists a notation system and framework for combinatorial objects similar to symbolic combinatorics called species [20]. Currently, Genfunlib provides support for the symbolic method only, although the possibility of implementing species instead and including a converter for specifications from the symbolic method to species was considered. It does appear as though a natural mapping exists between the sequence, set, and cycle constructions on the one hand, and the species of linear orders, sets, and cyclic permutations on the other. However, problems arise eventually. Whereas the pointing construction in the symbolic method corresponds, in unlabeled structures, to the power series operator $x D$, for a species $F$ the type generating series $\widetilde{F^{\circ}}(x)$ cannot be expressed in terms of $\widetilde{F}(x)$, but is given by

$$
\widetilde{F^{\circ}}(x)=x\left(\frac{\partial}{\partial x_{1}} Z_{F}\right)\left(x, x^{2}, x^{3}, \ldots\right) .
$$


Thus, in species, the pointing operation cannot correspond to any admissible construction in the symbolic method. This kind of subtle difference also exists between the substitution "construction" in the symbolic method and "operation" in species. Salvy et al. discuss perhaps less serious issues with converting the unlabeled multiset construction to the language of species [26].

\subsection{Asymptotics}

Flajolet et al. [10] define the class $\mathcal{E}$ of elementary functions as the set of functions containing the monomials 1 and $z$, and closed under the operations of $\{+, \times, Q, L, E\}$, where

$$
Q(f)=\frac{1}{1-f}, \quad L(f)=\log \frac{1}{1-f}, \quad E(f)=\exp (f) .
$$

They proceed to give algorithms that can be used to automatically extract Maclaurin coefficient asymptotics for functions in a subset of $\mathcal{E}$. Before going further here, we note that at this point some confusion can appear related to the terminology and notation used. First, even though when talking about asymptotics we need to think of generating functions as complex functions, for the purpose of computer algebra we really still compute with power series. That is, " $\exp (z)$ " cannot, strictly speaking, be called a function. Also, we actually want to talk about expressions built with the operations $+, \times, Q, L, E$, etc. we are talking about syntax, not semantics. Deciding whether a given expression semantically belongs in $\mathcal{E}$ is in general impossible, and not our main goal.

The class $\mathcal{E}$ is subdivided into three disjoint sets

$$
\mathcal{E}=\mathcal{E}_{\mathrm{AL}} \cup \dot{\cup} \mathcal{E}_{\text {entire }} \dot{\cup} \mathcal{E}_{\text {other }},
$$

where $\mathcal{E}_{\text {entire }}$ is the set of functions in $\mathcal{E}$ that are entire, and $\mathcal{E}_{\mathrm{AL}}$ is the functions 
with algebraic-logarithmic growth, meaning that $f \in \mathcal{E}_{\mathrm{AL}}$ if and only if $f$ has radius of convergence $0<r<\infty$ then there exist $\alpha \in \mathbb{R}, k \in \mathbb{Z}_{\geq 0}$ such that

$$
f(z) \sim \frac{1}{(1-z / r)^{\alpha}} \log ^{k} \frac{1}{1-z / r}, \quad \text { as } z \rightarrow r^{-} .
$$

The algorithm for computing asymptotic expansions of the Maclaurin coefficients of functions in $\mathcal{E}_{\mathrm{AL}}$ described by Flajolet et al. [10] was implemented in Combstruct for Maple and is now partly implemented in Genfunlib for Mathematica as the CoefLimit command. This command takes an aperiodic function $f \in \mathcal{E}_{\mathrm{AL}}$ and returns something asymptotically equivalent to the general term of the Maclaurin coefficient sequence of $f$. By saying that $f$ is aperiodic, we mean that $f(z)$ cannot be expressed as $z^{a} g\left(z^{b}\right)$ where $b>1$ and $a$ are integers, and $g \in \mathcal{E}_{\mathrm{AL}}$.

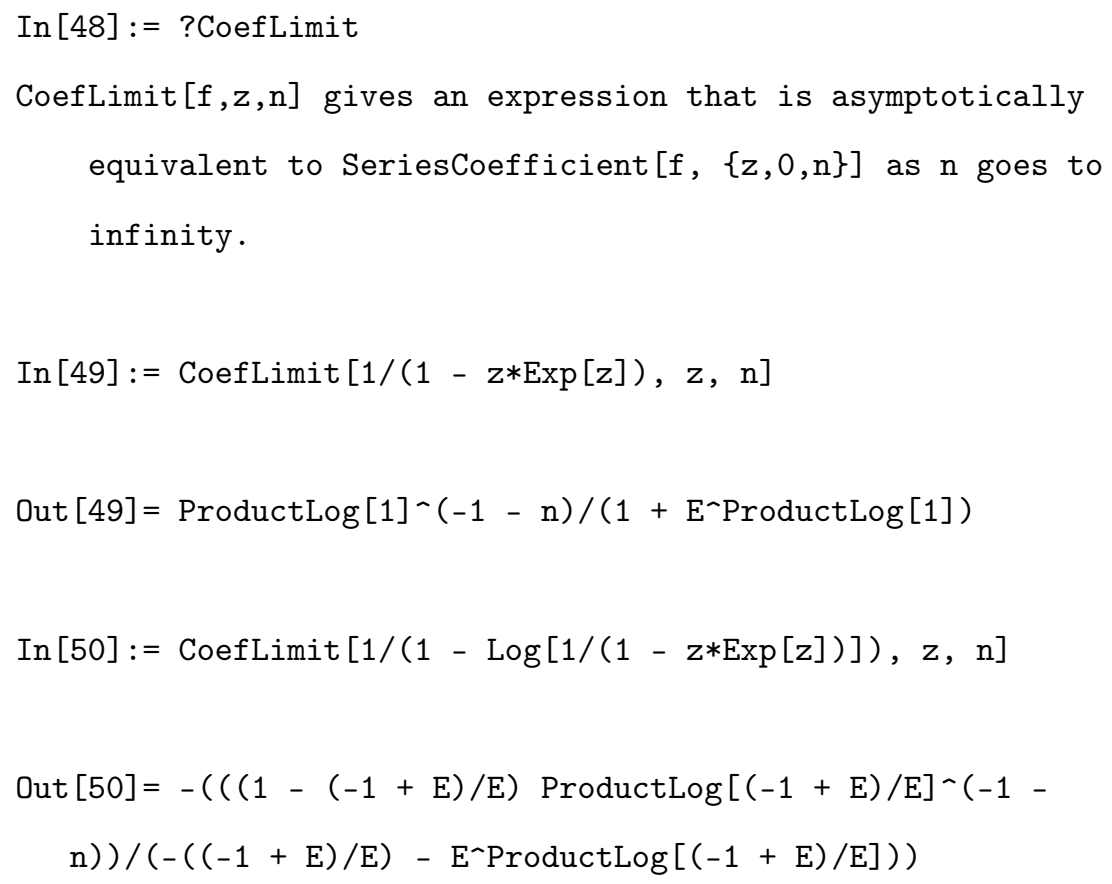


In the case where $f \in \mathcal{E}_{\mathrm{AL}}$ has positive radius of convergence $r$ and

$$
f(z) \sim \frac{1}{(1-z / r)^{\alpha}} \log ^{k} \frac{1}{1-z / r} \quad \text { as } z \rightarrow r^{-},
$$

where $\alpha \leq 0$, CoefLimit merely returns the exact expression for the coefficients, using Mathematica's SeriesCoefficient.

$\operatorname{In}[51]:=\operatorname{CoefLimit}[\log [1 /(1-z * \operatorname{Exp}[z])], z, n]$

Out $[51]=$ SeriesCoefficient $\left[\log \left[1 /\left(1-E^{\wedge} z z\right)\right],\{z, 0, n\}\right]$

In [52]:= \$Assumptions $=\mathrm{n}>1$;

In [53]:= CoefLimit $[1 /(1-z) \log [1 /(1-2 z)], z, n]$

Out $[53]=-\mathrm{I} * \mathrm{Pi}-2 \sim(1+\mathrm{n}) \operatorname{LerchPhi}[2,1,1+\mathrm{n}]$

Here LerchPhi $[2,1,1+\mathrm{n}]$ represents $\sum_{k \geq 0} 2^{k} /(k+n+1)$.

Mathematica note: The original algorithm [10] works for periodic generating functions and can obtain an asymptotic expansions of the form

$$
C \rho^{-n} n^{s} \log ^{k} n\left(1+O\left(\frac{1}{\log n}\right)\right)
$$

However, in Mathematica asymptotic series are represented with SeriesData objects, and these only support big-Oh terms of the form $O\left(n^{k}\right)$. Thus, CoefLimit does not give big-Oh terms, merely the dominant term. Since periodic generating functions have zeroes in their coefficients, the coefficients are not asymptotically equivalent to anything, so Genfunlib cannot give an asymptotic 
A general way to specify a generating function is to define it implicitly by a power series equation. Genfunlib provides the TreeAsymptot command for computing the coefficient asymptotics of generating functions $f(z)$ satisfying equations of the form

$$
f(z)=z \phi(f(z))
$$

where $\phi \in \mathcal{E}_{\mathrm{AL}}$ satisfies $\phi(z) \neq c_{0}+c_{1} z$ and $\phi(0) \neq 0$. In this case, we can apply Theorem VI.6 [11] to derive the asymptotics of $\left[z^{n}\right] f(z)$ provided we can compute the minimum solution $\tau$ to the characteristic equation

$$
\phi(\tau)-\tau \phi^{\prime}(\tau)=0, \quad \tau>0,
$$

and the periodicity of $\phi$. The value of $\tau$ can be computed by Mathematica's built-in equation solver, and we can obtain the necessary information about the period of $\phi$ via the "Reduction" algorithm from Flajolet et. al [10].

The TreeAsymptot command uses this method to give an asymptotic expression consisting of a main estimate and a big $\mathrm{O}$ term.




The case where $\phi(z)$ is periodic is allowed.

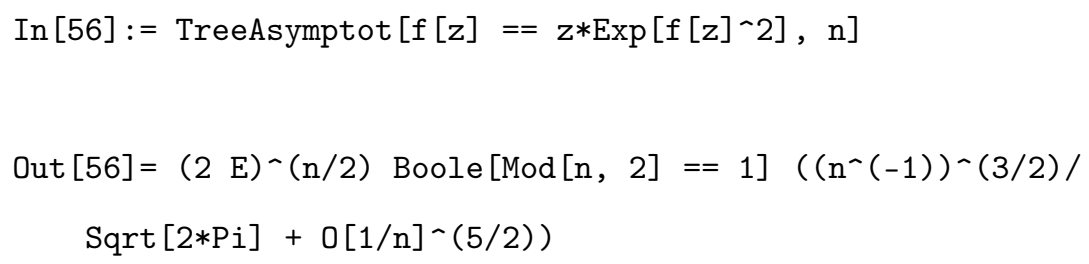

Flajolet and Sedgewick [11] give an algorithm for obtaining coefficient asymptotics of generating functions defined by algebraic equations. This surely useful algorithm has so far not been implemented in any computer algebra system.

\section{Genfunlib in action}

\subsection{Mathematics contest problems}

Elementary problems from combinatorics are often chosen for high school math contests. This subsection contains a selection of contest-style problems and shows how Genfunlib can help to solve them; more contest problems solved with the aid of a computer algebra system can be found in an article by Dumas and Salvy [8].

Problem 1. How many arrangements are there of the letters in the word MATHEMATICS? [30]

Solution: As Zeilberger says [12], "Enumerating specific finite sets is no longer considered mathematics. A genuine mathematical fact has to incorporate infinitely many facts, and the generic enumeration problem is to enumerate not just one set but all the sets in an infinite family." In particular, this problem can be solved easily "by computer" simply by performing a brute-force enumeration (in a math contest, this method is too slow). However, Genfunlib could also be used. 
The word MATHEMATICS contains 11 letters. If we create sequences of 11 atoms (1), we can associate the label of the $i$ th atom with the position of the $i$ th letter of MATHEMATICS in an arrangement of the letters. For example,

$$
\text { ( 8), (4), (5), (2), (7), (1), (3), 10, (9), (11), (6) }
$$

corresponds to the arrangement MHAATSEMITC. The problem is that not all letters in MATHEMATICS are distinct, so not every different labeled sequence corresponds to a different arrangement. We fix this by creating sequences of sets of atoms, where there is one set for each different letter in MATHEMATICS. This way, each set of positions of a letter is counted, not each sequence of positions.

Our specification of the class $\mathcal{M}$ of rearrangements of MATHEMATICS is

$$
\begin{aligned}
\mathcal{M} & \left.\left.=\operatorname{SET}_{2}(1)\right)^{3} \times \operatorname{SET}_{1}(1)\right)^{5} \\
& \left.=\operatorname{SET}_{2}(1)\right)^{3} \times(1)^{5}
\end{aligned}
$$

With Genfunlib, we convert this to an exponential generating function $\mathrm{m}[\mathrm{z}]$ where the coefficient of $z^{11}$ multiplied by 11 ! is the solution to the problem.

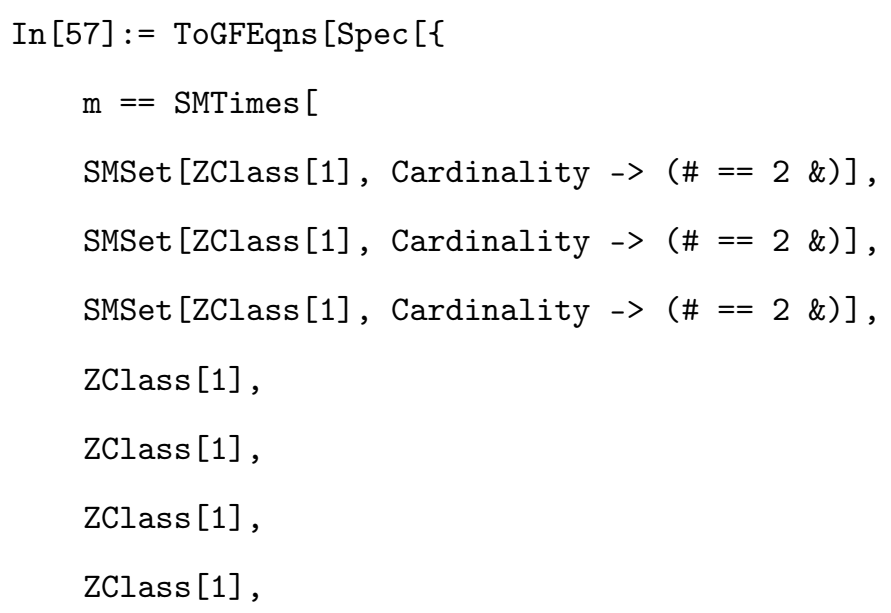




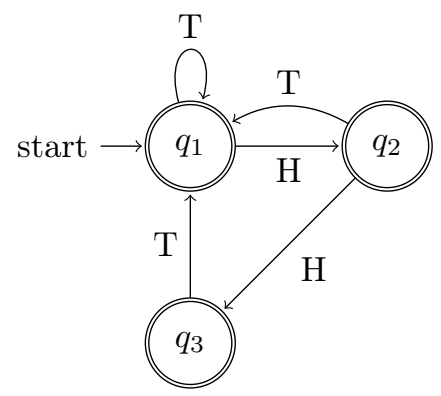

Figure 6: An NFA $N$ for the allowed sequences of coin tosses in Problem 2.

ZClass [1]

]\}, True], z] /. z[1] $->z$

Out $[57]=\left\{\mathrm{m}[\mathrm{z}]==\mathrm{z}^{\wedge} 11 / 8\right\}$

From here we observe that the solution is

$\operatorname{In}[58]:=11 ! / 8$

Out $[58]=4989600$

Problem 2. A fair coin is to be tossed ten times. Find the probability that heads never occur on three consecutive tosses. (This is a modified version of AIME $1990 \# 9$, a typical Fibonacci problem [31].)

Solution: Say the coin is tossed $n$ times. Then we can represent the sequence of tosses as an $n$-word over the alphabet $\{H, T\}$. Let $N$ be the NFA in Figure 6 . Then the language accepted by $N$ is the set of words representing coin tosses in which heads never occur on three consecutive tosses. Using Genfunlib, we can convert $N$ to an equivalent regular expression.

In [59]:= ToRegex [NFA $[3,\{" H ", " T "\},\{$

$\{\{2\},\{1\},\{\}\}$, 


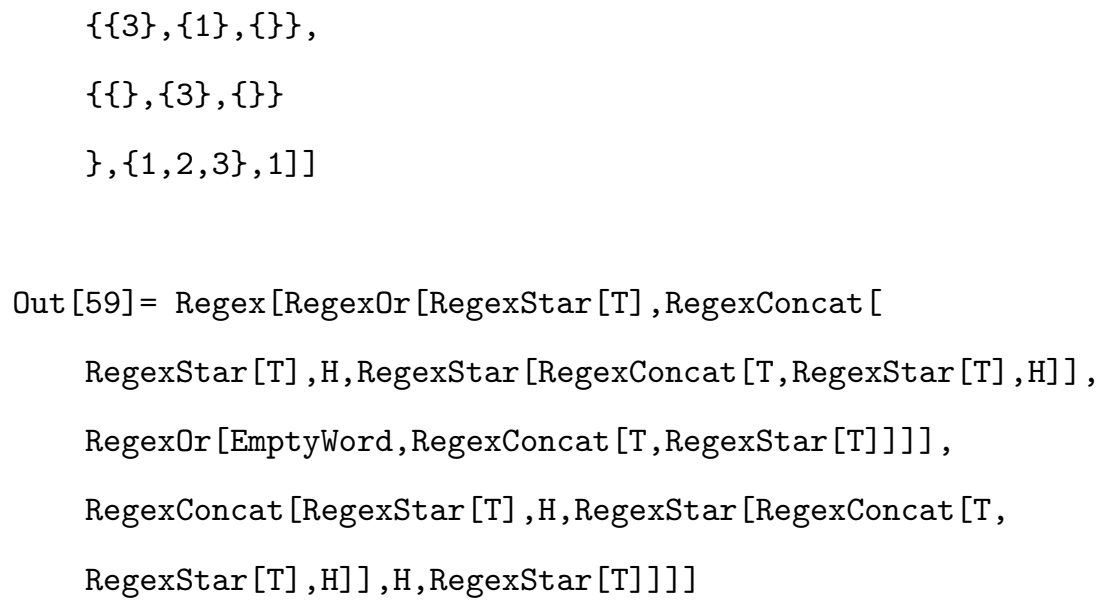

Now, we obtain the generating function of this regular expression to find the number of words of length 10 .

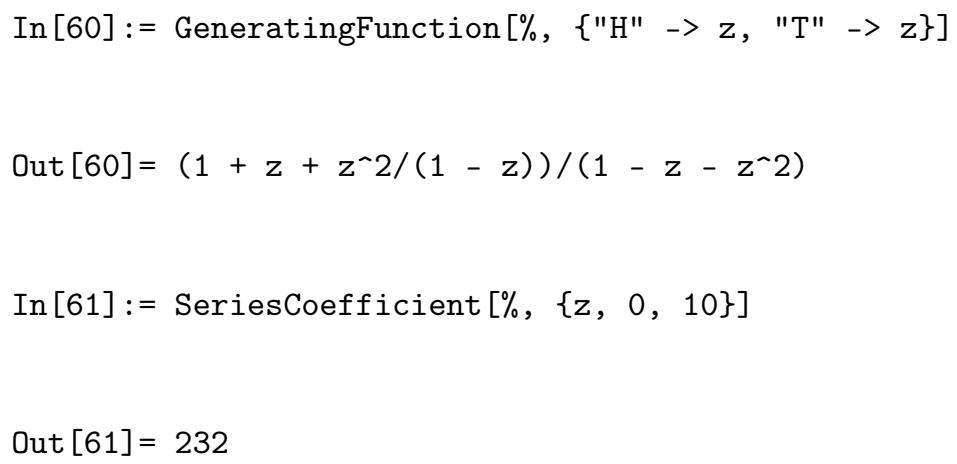

Thus, the answer is $232 / 2^{10}=29 / 128$.

Problem 3. Find a recursion for the number of partitions of an $n$-set [9].

First we can note that the combinatorial class $\mathcal{P}$ of set partitions is defined

$$
\left.\mathcal{P}=\operatorname{SMSET}\left(\operatorname{SMSET}_{\geq 1}(1)\right)\right)
$$

That is, a set partition is simply a set of nonempty sets. With Genfunlib we can get the exponential generating function $P(z)$ for the class $\mathcal{P}$, represented by $\mathrm{p}[\mathrm{z}]$. 


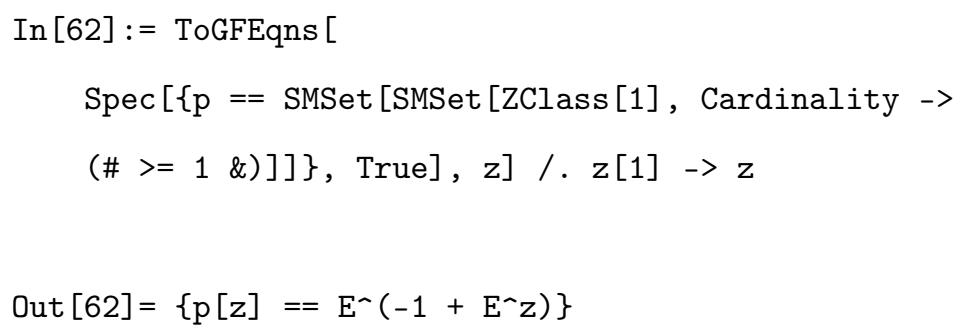

This is actually an explicit equation for the generating function $p[z]$. To arrive at a recurrence relation, we logarithmically differentiate both sides and solve for $\mathrm{p}[\mathrm{z}]$

$\operatorname{In}[63]:=\operatorname{Solve}\left[D[\log [p[z]], z]==D\left[\log \left[E^{\wedge}\left(-1+E^{\wedge} z\right)\right], z\right], p[z]\right]$

Out $[63]=\left\{\left\{\mathrm{p}[\mathrm{z}]->\mathrm{E}^{\wedge}-\mathrm{z} \mathrm{p}^{\prime}[\mathrm{z}]\right\}\right\}$

Using Genfunlib's enhancement to SeriesCoefficient, we extract coefficients of the right hand side.

In $[64]:=$ SeriesCoefficient $\left[E^{\wedge}-z p^{\prime}[z],\{z, 0, n\}\right]$

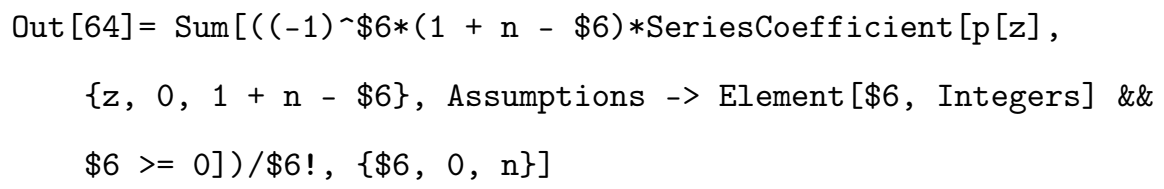

If we let $p_{n}=n !\left[z^{n}\right] P(z)$, we can use the above result to arrive at the relation

$$
p_{0}=1, \quad p_{n}=p_{n-1}-\sum_{j=1}^{n-1}\left(\begin{array}{c}
n-1 \\
j
\end{array}\right)(-1)^{j} p_{n-j} .
$$

Problem 4. Prove that the number of binary $n$-words with exactly $m 01-$ blocks is $\left(\begin{array}{c}n+1 \\ 2 m+1\end{array}\right)[9]$.

Solution: The way we attack this problem is defining the NFA $N_{b}$ in Figure 7. The language that $N_{b}$ accepts is all words over the alphabet $\{0,1, b\}$ where 


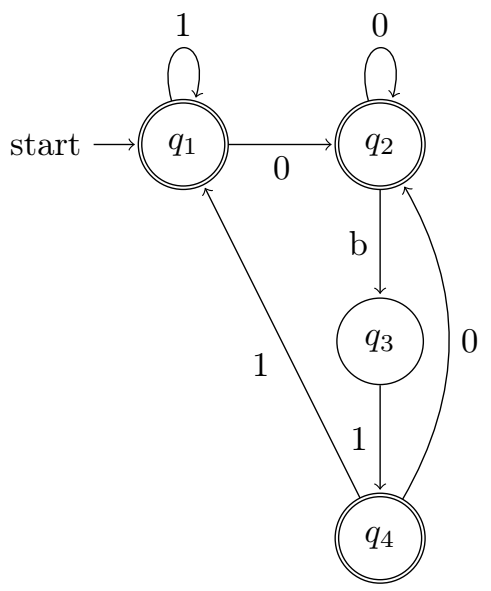

Figure 7: An NFA $N_{b}$ that marks 01 blocks with $b$.

the letter $b$ only appears as part of the subword $0 b 1$, and the subword 01 never appears. Counting the number of words with $n$ letters 0 and 1 and $m$ occurrences of $b$ will solve the problem.

We define $\mathrm{nfab}$ to be $N_{b}$ from Figure 7 with Genfunlib.

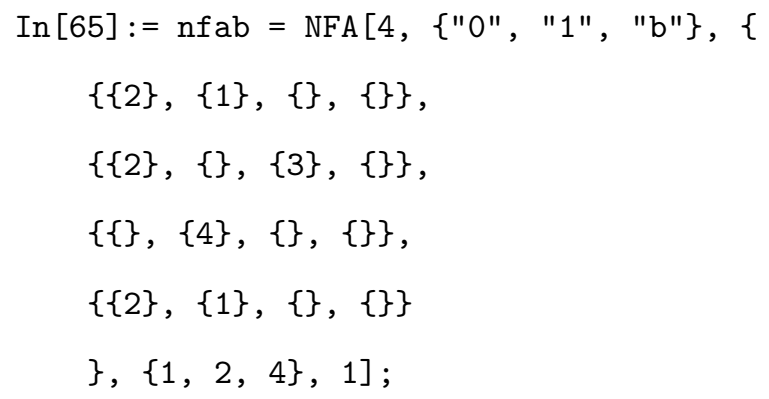

We obtain a generating function from nfab, with $u$ marking 0 and 1 , and $v$ marking $b$.

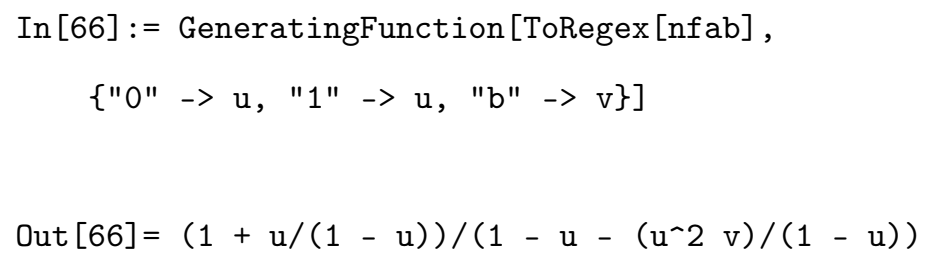


Now, we extract the coefficient of $v^{m}$.

$\operatorname{In}[67]:=$ \$Assumptions $=\mathrm{m}>=0$;

$\operatorname{In}[68]:=$ SeriesCoefficient $[1 /(1+u(-2+u-u v)),\{v, 0, m\}]$

Out [68] $=\left(u^{\wedge} 2 /(-1+u) \sim 2\right) \wedge(1+m) / u^{\wedge} 2$

Lastly we compare this to $\sum_{n \geq 2 m}\left(\begin{array}{c}n+1 \\ 2 m+1\end{array}\right) u^{n}$.

$\operatorname{In}[69]:=\operatorname{Out}[68]==\operatorname{Sum}\left[\right.$ Binomial $[n+1,2 m+1] u^{\wedge} n$,

\{n, 2 m, Infinity $\}] / /$ FullSimplify

Out $[69]=$ True

This may seem like an indirect route to the result. The explanation is that, as long as we want to use Mathematica and Genfunlib as much as possible, the most direct route sometimes does not work but something more creative does.

Problem 5. How many $n$-words from the alphabet $\{0,1,2\}$ are such that the neighbors differ by at most 1? [9]

Solution: One can see that this is a regular language accepted by the NFA $N_{d}$ shown in Figure 8. We define $N_{d}$ in Genfunlib as nfadif.

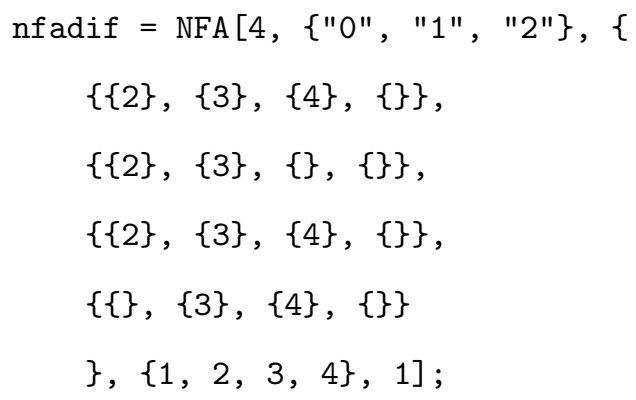




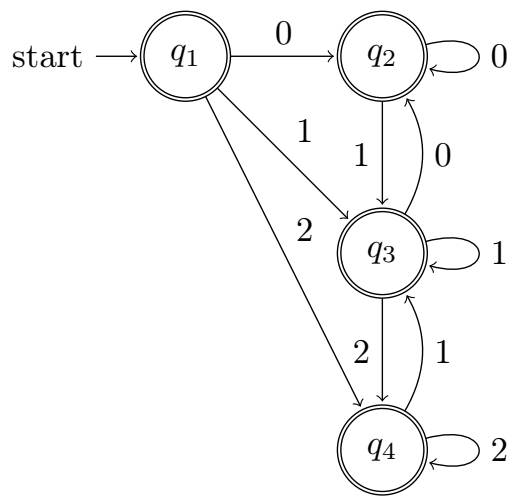

Figure 8: The NFA $N_{d}$ that accepts words from the alphabet $\{0,1,2\}$ such that neighboring letters differ by at most 1 .

All that remains in order to count the $n$-words is to obtain the generating function and extract coefficients.

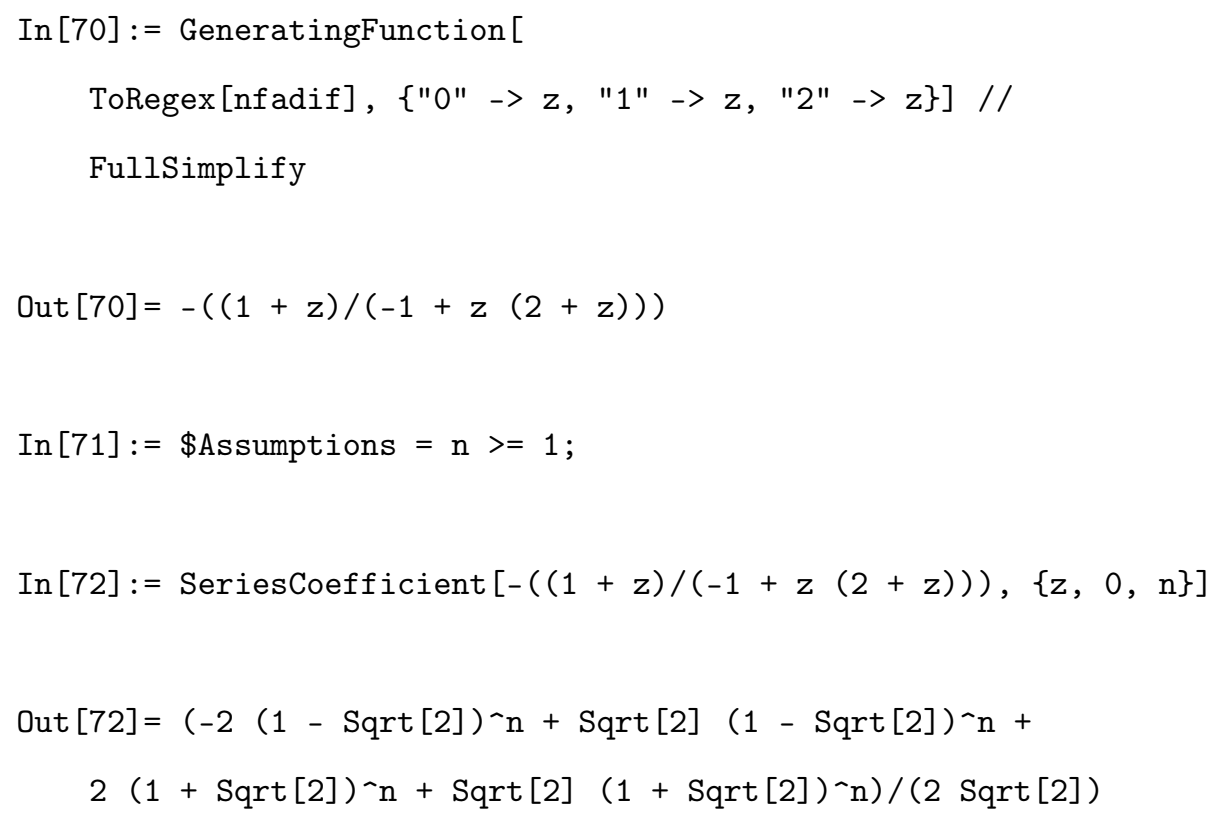

Simplified and in standard mathematical notation, this says that there are

$$
\frac{1}{2}\left((1-\sqrt{2})^{n+1}+(1+\sqrt{2})^{n+1}\right)
$$


$n$-words from the alphabet $\{0,1,2\}$ such that neighboring letters differ by at most 1 .

Problem 6. If $n$ people sit around a circular table, how many of the $n$ ! arrangements are distinct, i.e., do not have the same neighboring relations?

Solution: The first thing to note about this problem is that it appears to be ambiguous. Is the neighboring relation who is sitting next to whom, or who is sitting to the left of whom? Let us solve the problem for the latter case. It turns out that the final answers simply differ by a factor of 2 .

We represent the seating arrangements by the combinatorial class $\mathcal{C}=$ CyC (1)). That is, the objects in $\mathcal{C}$ are labeled cycles. With Genfunlib, we can find $c[z]$, the generating function of $\mathcal{C}$, and extract coefficients.

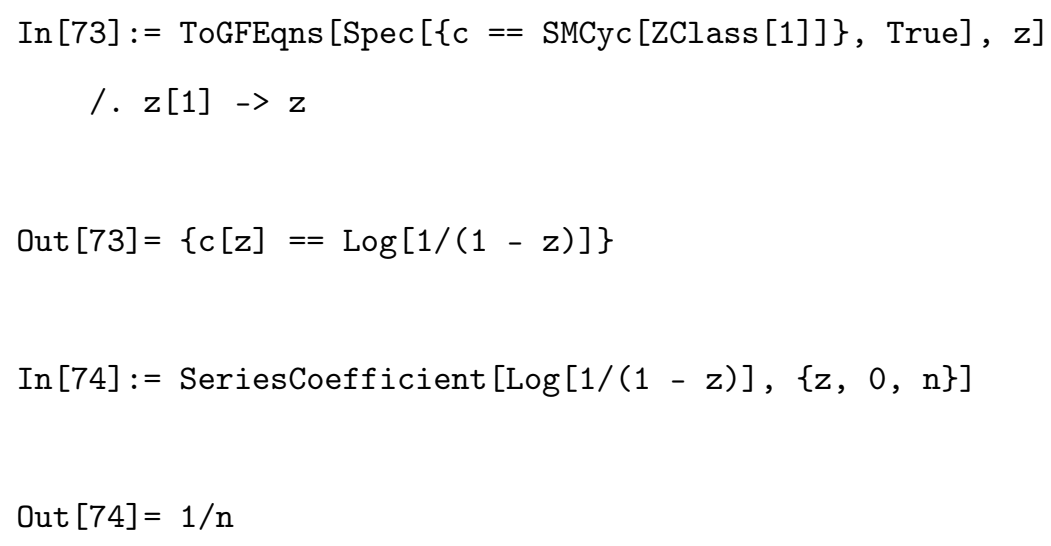

Since $c[z]$ is an exponential generating function, the number of distinct arrangements is $n ! / n=(n-1) !$.

Problem 7. A permutation $p$ of the set $\{1, \ldots, n\}$ is called an involution if $p \circ p=$ Id. Find a recursion for the number $t_{n}$ of involutions of $\{1, \ldots, n\}$. Also find a closed formula in the form of a sum [9]. 
Solution: The functional graph of an involution is a set of 1 - and 2-cycles. So we define the class $\mathcal{G}$ by

$$
\left.\mathcal{G}=\operatorname{SET}\left(\mathrm{CYC}_{\leq 2}(1)\right)\right)
$$

With Genfunlib, we find the generating function $\mathrm{g}[\mathrm{z}]$ for $\mathcal{G}$.

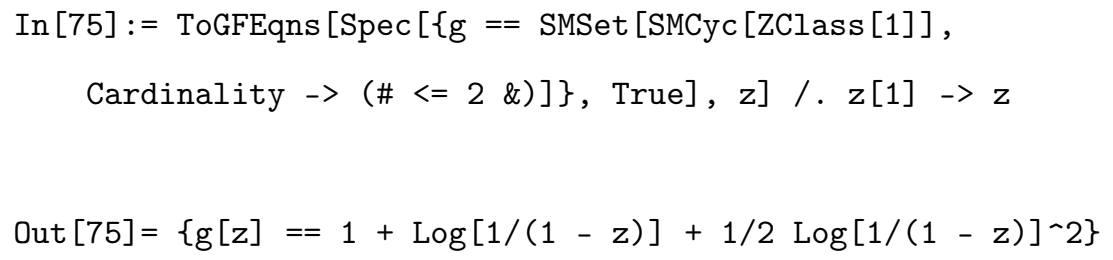

From this expression for $\mathrm{g}[\mathrm{z}]$, Mathematica can produce a recurrence relation for $t_{n}$, which we call tn.

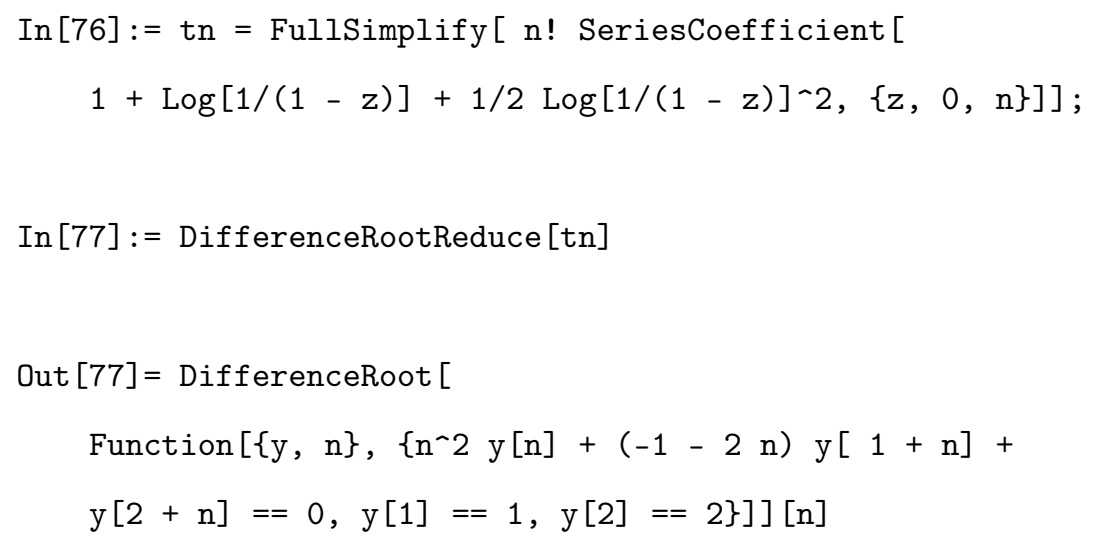

That is, $t_{1}=1, t_{2}=2$, and, for $n \geq 3$,

$$
n^{2} t_{n}-(2 n+1) t_{n+1}+t_{n+2}=0 .
$$

Now, finding a sum for $t_{n}$ should be easy since we have the generating function $g[z]$. There is a small problem, though, which is that Mathematica tries to "simplify" the sum into something else as soon as it is obtained. To avoid this, we 
replace $\log (1 /(1-z))$ in $g[z]$ with the placeholder $f(z)$, then extract coefficients from $g[z]$ in terms of the coefficients of $f(z)$, using Genfunlib's enhancements to Mathematica's SeriesCoefficient. Then we replace coefficients of $f(z)$ with the coefficients of $\log (1 /(1-z))$, and we are done.

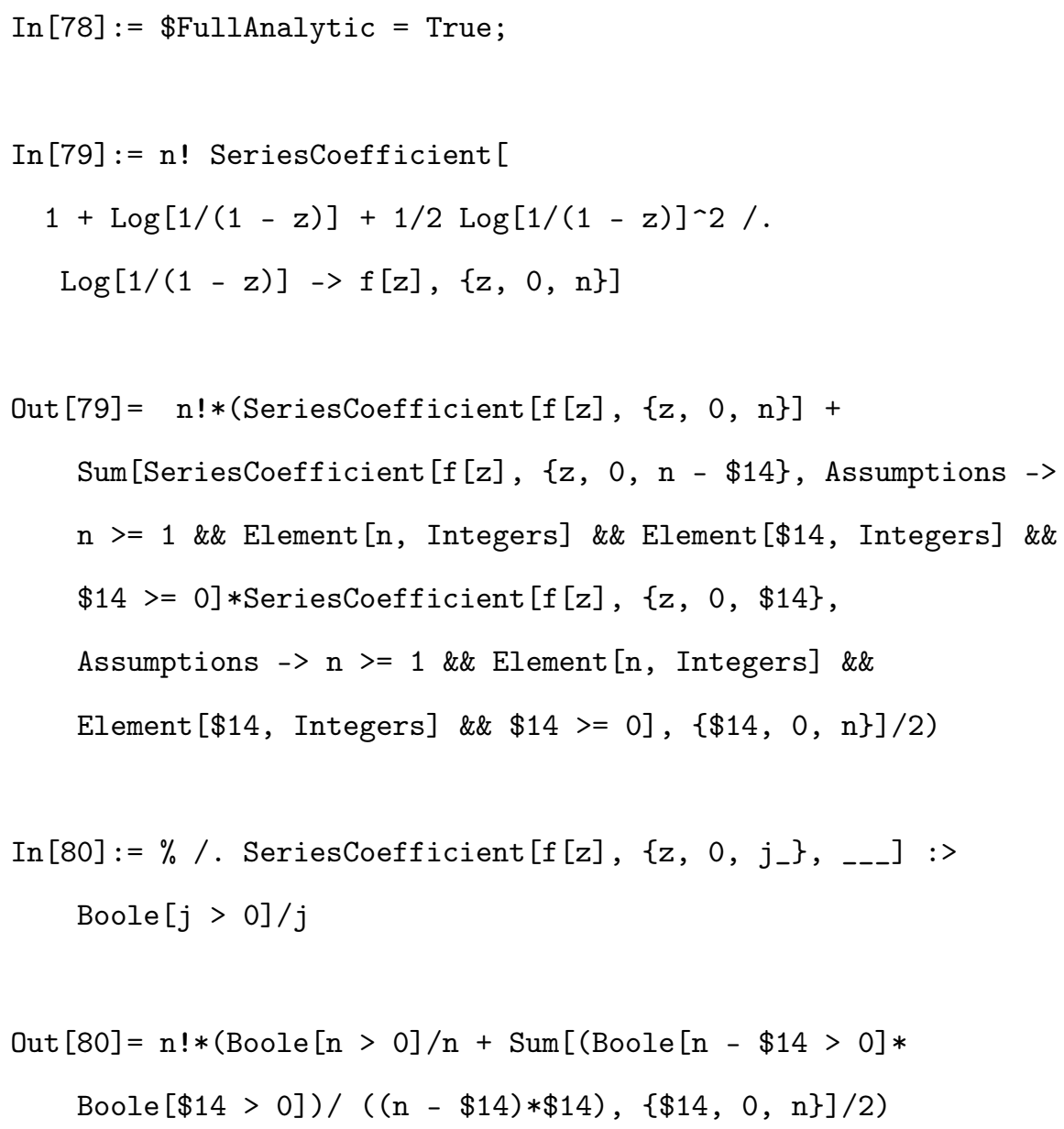

The result is a bit cluttered, but after a little inspection, it says that, for $n \geq 1$

$$
t_{n}=n !\left(\frac{1}{n}+\frac{1}{2} \sum_{j=1}^{n-1} \frac{1}{j(n-j)}\right)
$$


Problem 8. Let $f(n)$ be the number of $n$-words without neighboring zeros from the alphabet $\{0,1,2\}$. Find a recursion and a formula for $f(n)$.

Solution: To specify the language of interest, we define the grammar $G$ with the following productions.

$$
\begin{aligned}
& A \rightarrow 1 \cdot A \\
& A \rightarrow 2 \cdot A \\
& A \rightarrow 0 \cdot B \\
& B \rightarrow 1 \cdot A \\
& B \rightarrow 2 \cdot A \\
& A \rightarrow \epsilon \\
& B \rightarrow \epsilon
\end{aligned}
$$

In Genfunlib, we can define the corresponding grammar oneZero and compute the generating function.

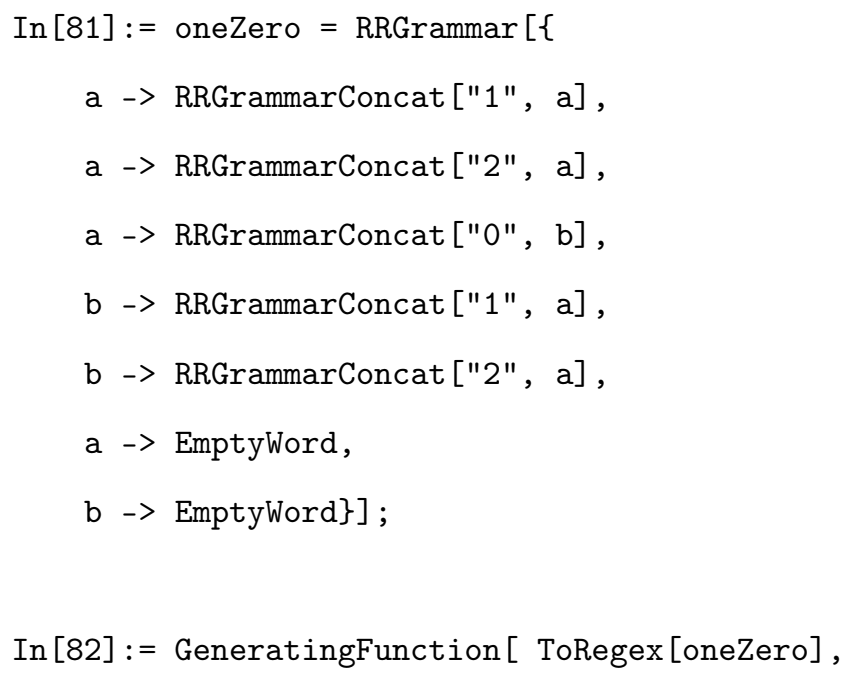






Figure 9 shows a digraph with labeled vertices corresponding to onezero, which was created with Genfunlib's ToDigraph command and edited slightly for clarity.

Finding a formula for $f(n)$ is now as simple as extracting coefficients using Mathematica's SeriesCoefficient command.

In [83] := SeriesCoefficient $[\%,\{z, 0, n\}]$

$\operatorname{Out}[83]=\left(-2(1-\operatorname{Sqrt}[3])^{\wedge} \mathrm{n}+\operatorname{Sqrt}[3](1-\operatorname{Sqrt}[3])^{\wedge} \mathrm{n}+\right.$

$\left.2(1+\operatorname{Sqrt}[3])^{\wedge} n+\operatorname{Sqrt}[3](1+\operatorname{Sqrt}[3])^{\wedge} n\right) /(2 \operatorname{Sqrt}[3])$

After some simplification, we have that

$$
f(n)=\frac{1}{6}(3+2 \sqrt{3})(1+\sqrt{3})^{n}-\frac{1}{6}(2 \sqrt{3}-3)(1-\sqrt{3})^{n} .
$$

Obtaining a recurrence relation is as easy as calling a Mathematica command called DifferenceRootReduce.

In [84]:= DifferenceRootReduce $[\%, \mathrm{n}] / /$ FullSimplify

Out $[84]=\operatorname{DifferenceRoot~}[$ Function $[\{\mathrm{y}, \mathrm{n}\},\{-2 \mathrm{y}[\mathrm{n}]-$

$2 \mathrm{y}[1+\mathrm{n}]+\mathrm{y}[2+\mathrm{n}]==0, \mathrm{y}[0]==1, \mathrm{y}[1]==3\}]][\mathrm{n}]$

That is, $f(0)=1, f(1)=3$, and, for $n \geq 2$,

$$
f(n+2)=2 f(n)+2 f(n+1)
$$




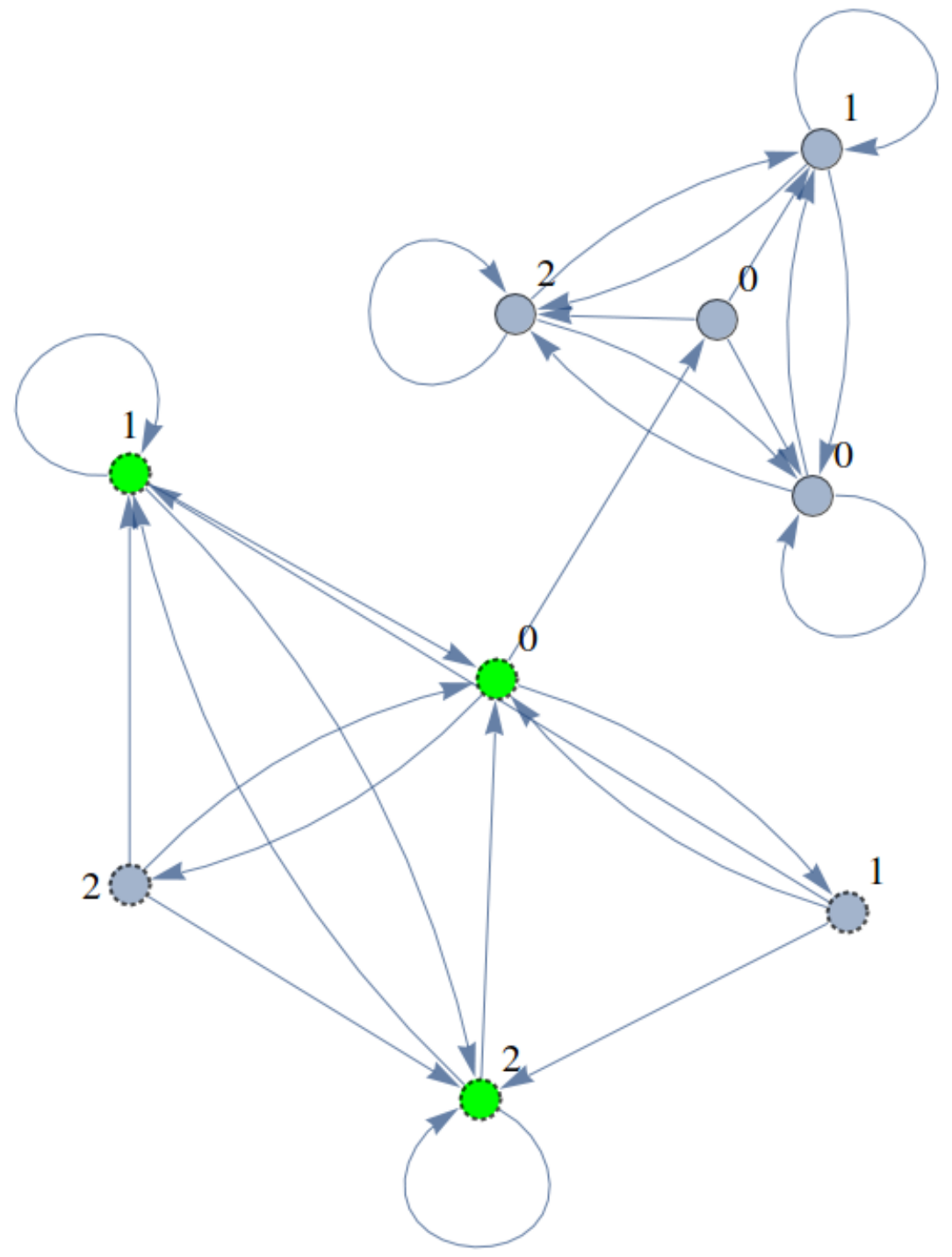

Figure 9: A digraph with labeled vertices that is equivalent to the grammar $G$ from Problem 8, which accepts words in $\{0,1,2\}^{*}$ that avoid the subword 00. This non-minimal digraph was generated with the ToDigraph command and plotted using Mathematica's built-in functionality. Green vertices are start vertices, and vertices with dotted borders are accepting vertices. 

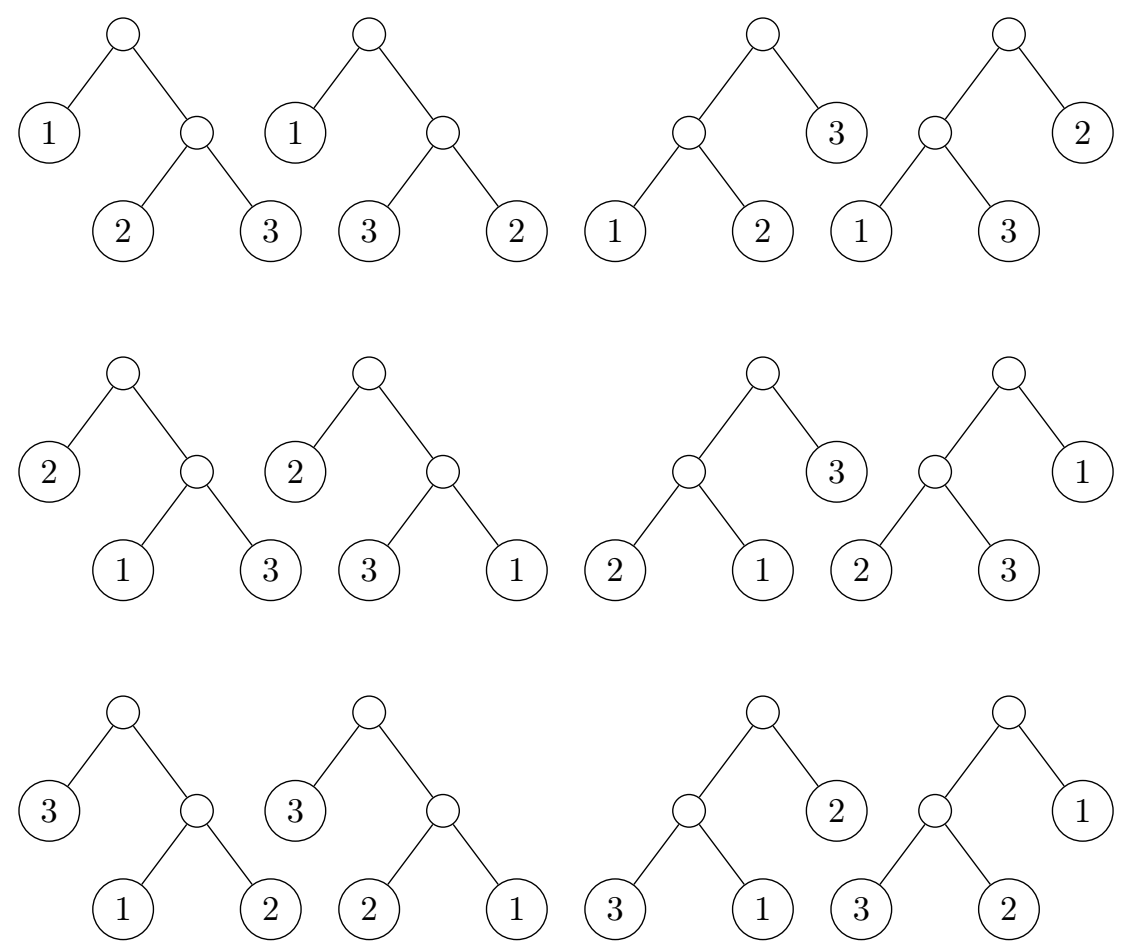

Figure 10: The 12 binary trees with 3 labeled leaves.

Problem 9. How many binary trees with $n$ labeled leaves are there? [9]

\section{Solution:}

We assume that "binary trees" are rooted plane trees such that every node has either 0 or 2 children. We assume that these leaf-labeled binary trees are binary trees whose leaves are uniquely labeled. The task is to count how many leaf-labeled binary trees there are with $n$ leaves. Figure 10 shows all leaf-labeled binary trees with 3 leaves. 
First we define a combinatorial specification for the classes of leaves, $\mathcal{L}$, inner nodes $\mathcal{I}$, and trees $\mathcal{T}$.

$$
\begin{aligned}
& \mathcal{L}=1 \\
& \mathcal{I}=\mathcal{E} \\
& \mathcal{T}=\mathcal{L}+\mathcal{T} \times \mathcal{I} \times \mathcal{T}
\end{aligned}
$$

In Genfunlib, we can convert this specification to equations in the exponential generating functions $1[z]$ for $\mathcal{L}, i[z]$ for $\mathcal{I}$, and $t[z]$ for $\mathcal{T}$.

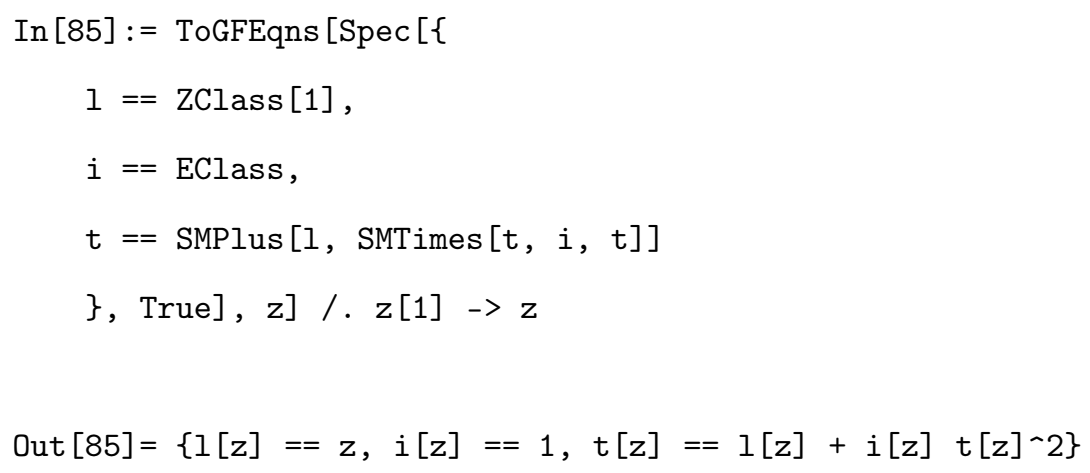

We use Mathematica's Eliminate to provide us with an equation in just our generating function of interest, $t[z]$.

$\operatorname{In}[86]:=$ eqn $=$ Eliminate $[\%,\{1[z], i[z]\}]$

Out $[86]=(1-t[z]) t[z]==z$

The power series equation $(1-T(z)) T(z)=z$ has two solutions in general, but only one with nonnegative coefficients: $(1 / 2)(1-\sqrt{1-4 z})$. Extracting coefficients with Mathematica's SeriesCoefficient and multiplying by $n$ ! gives the final answer for the number of leaf-labeled binary trees with $n$ leaves. 


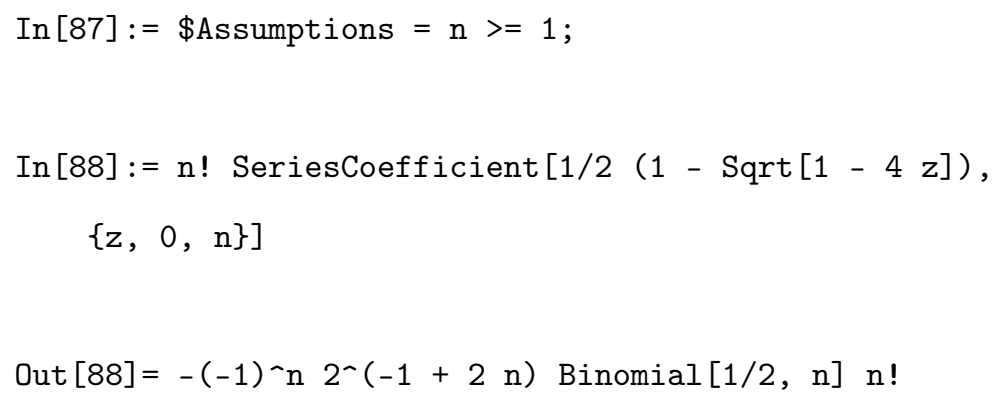

Mathematica reports here that the formula is

$$
-(-1)^{n} 2^{2 n-1} n !\left(\begin{array}{c}
\frac{1}{2} \\
n
\end{array}\right)
$$

which is just a more cumbersome form of the expected result

$$
\frac{(2(n-1)) !}{(n-1) !}
$$

We note that one way to come up with the specification in this solution is to find the counting sequence for leaf-labeled binary trees in the Encyclopedia of Combinatorial Objects at http://algo.inria.fr/ecs. It is entry number 51, "Labelled Plane Binary Trees". The Encyclopedia shows the unlabeled Maplesyntax specification $\{\mathrm{S}=\operatorname{Union}(\mathrm{Z}, \operatorname{Prod}(\mathrm{S}, \mathrm{S}))\}$, which we can use to get a specification in Genfunlib via the ToGenfunlibSpec command.

$\operatorname{In}[89]:=\operatorname{ToGenfunlibSpec}["\{\mathrm{~S}=\operatorname{Union}(\mathrm{Z}, \operatorname{Prod}(\mathrm{S}, \mathrm{S}))\} "$, False $]$

Out $[89]=\operatorname{Spec}[\{\mathrm{ss}==\operatorname{SMPlus}[\mathrm{ZClass}[1], \operatorname{SMTimes}[\mathrm{ss}, \mathrm{ss}]]\}$, False $]$

Problem 10. Let $k$ and $n$ be integers such that $1 \leq k \leq n$. Consider all finite sequences of positive integers with sum $n$. Suppose that the term $k$ occurs $T(n, k)$ times in all these sequences. Find $T(n, k) .[9]$ 
Solution: We start with a specification of the class $\mathcal{S}$ of integer compositions where $\mathcal{Z}_{1}$ marks the number of parts equal to $k$, and $\mathcal{Z}_{2}$ marks the sum of all parts.

$$
\begin{aligned}
\mathcal{P}_{1} & =\operatorname{MSET}_{\geq 1,<k}\left(\mathcal{Z}_{2}\right) \\
\mathcal{P}_{2} & =\operatorname{MSET}_{k}\left(\mathcal{Z}_{2}\right) \times \mathcal{Z}_{1} \\
\mathcal{P}_{3} & =\operatorname{MSET}_{>k}\left(\mathcal{Z}_{2}\right) \\
\mathcal{S} & =\operatorname{SEQ}\left(\mathcal{P}_{1}+\mathcal{P}_{2}+\mathcal{P}_{3}\right) .
\end{aligned}
$$

Here, $\mathcal{P}_{1}$ is the set of parts smaller than $k$ but no smaller than $1 ; \mathcal{P}_{2}$ is the singleton set containing the part of size $k$, marked with $\mathcal{Z}_{1}$; and $\mathcal{P}_{3}$ is the set of parts larger than $k$. Now, we ultimately wish to count the number of occurrences of $k$ in all compositions of $n$. We do this using the symbolic method by defining a class $\mathcal{F}$ each of whose elements corresponds to an occurrence of $k$ in a composition of $n$. It suffices to define $\mathcal{F}$ as the class obtained by taking $\mathcal{S}$ and pointing separately at each atom $\mathcal{Z}_{1}$, then substituting $\mathcal{E}$ for $\mathcal{Z}_{1}$ so that the objects in $\mathcal{F}$ are only weighted by $\mathcal{Z}_{2}$, which marks the total size of the composition.

$$
\mathcal{F}=(\Theta \mathcal{S}) \circ \mathcal{E}
$$

We give the whole specification to Genfunlib and compute the corresponding system of ordinary generating function equations. (We assume $k \geq 2$ for simplicity; the case $k=1$ can easily be handled separately.)

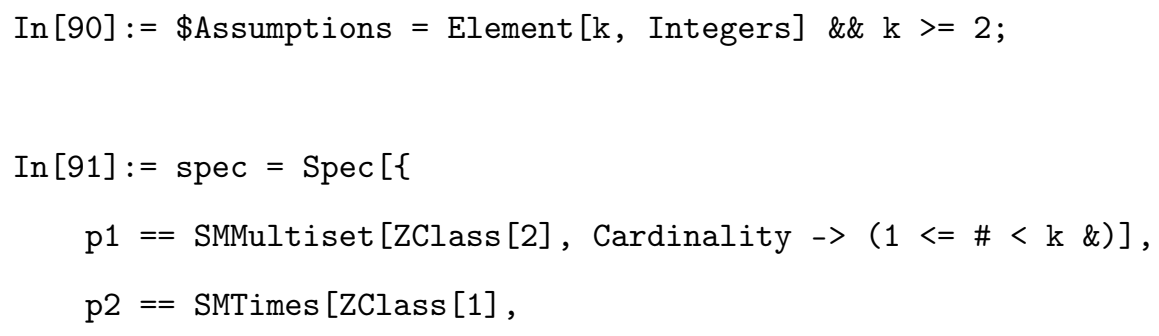


SMMultiset [ZClass [2], Cardinality $->(\#==\mathrm{k} \&)]$ ], p3 == SMMultiset [ZClass[2], Cardinality $->(\#>\mathrm{k} \&)]$, $\mathbf{s}==\operatorname{SMSeq}[\operatorname{SMP} 1 \mathrm{us}[\mathrm{p} 1, \mathrm{p} 2, \mathrm{p} 3]]$, $f==\operatorname{SMSub}[$ SMPointing $[\mathrm{s}]$, EClass $]$ \}, False] ;

In $[92]:=$ eqns $=$ ToGFEqns $[$ spec, z];

The full system of equations stored in eqns is long, so we do not show it in full. Let $S\left(z_{1}, z_{2}\right)$ be the ordinary generating function for $\mathcal{S}$ and let $F\left(z_{2}\right)$ be the ordinary generating function for $\mathcal{F}$. We solve the system to obtain $S\left(z_{1}, z_{2}\right)$, represented in Genfunlib by s[z[1], z[2]].

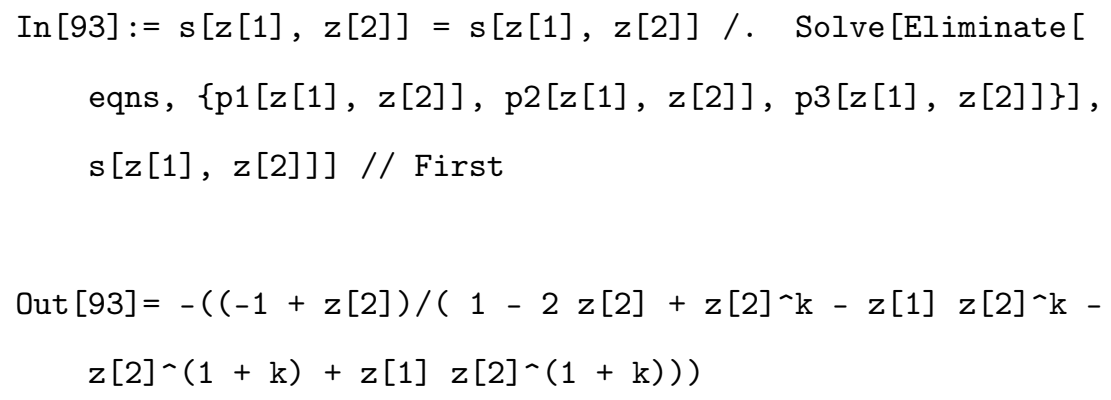

We manually compute $F\left(z_{2}\right)$, represented by f [z [2] ], using s [z [1] ] z [2] ] and the equation

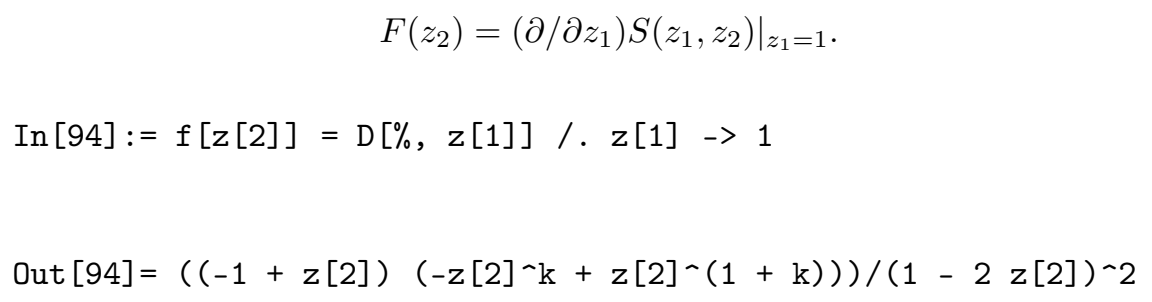


Extracting coefficients from $F\left(z_{2}\right)$ with a command built into Mathematica, we get the final result:

$$
T(n, k)= \begin{cases}2^{-k+n-2}(-k+n+3) & \text { if } k \leq n-2, \\ 2^{n-k} & \text { if } k=n-1, \\ 2^{n-k}(-k+n+1) & \text { if } k=n .\end{cases}
$$

Problem 11. An $n$-term sequence $\left(x_{1}, x_{2}, \ldots, x_{n}\right)$ in which each term is either 0 or 1 is called a binary sequence of length $n$. Let $a_{n}$ be the number of binary sequences of length $n$ containing no three consecutive terms equal to $0,1,0$ in that order. Let $b_{n}$ be the number of binary sequences of length $n$ that contain no four consecutive terms equal to $0,0,1,1$ or $1,1,0,0$ in that order. Prove that $b_{n+1}=2 a_{n}$ for all positive integers $n$. (USA Mathematical Olympiad $1996 \# 4$ )

Solution: We use the letters $a$ and $b$ to represent 0 and 1 , respectively, so $n$-term binary sequences become $n$-words over the alphabet $\{a, b\}$.

In an actual math contest, it would be most efficient to solve this problem using a bijection. However, a brute-force solution is feasible if it puts Genfunlib to work.

We define a regular expression $r_{1}$ that matches words that avoid $a b a$. In Genfunlib we represent this regular expression by $r 1$, which is set to the complement of the regular expression $(a \mid b)^{*} a b a(a \mid b)^{*}$ with respect to the alphabet $\{a, b\}$.

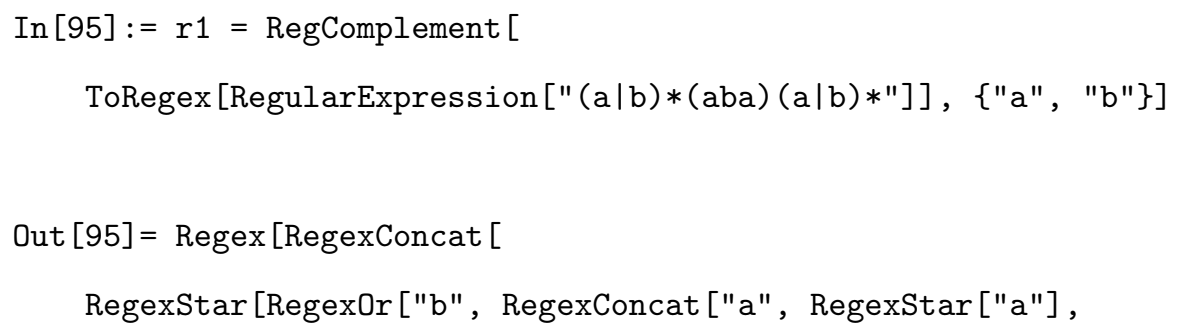


"b", "b"]]], RegexOr[EmptyWord, RegexConcat ["a",

RegexStar["a"], RegexOr[EmptyWord, "b"]]]]

We represent the generating function $\sum_{n \geq 0} a_{n} z^{n}$ by $\mathrm{f} 1$.

In $[96]:=f 1=$ FullSimplify[GeneratingFunction $[r 1$,

$\{" \mathrm{a} "->\mathrm{z}, " \mathrm{~b} "->\mathrm{z}\}]\}$

Out [96]:: $\left(1+z^{\wedge} 2\right) /\left(1+z\left(-2+z-z^{\wedge} 2\right)\right)$

We define $\mathrm{r} 2$ as the complement of $(a \mid b)^{*} a a b b(a \mid b)^{*}$, and we define $\mathrm{r} 3$ as the complement of $(a \mid b)^{*} b b a a(a \mid b)^{*}$.

$\operatorname{In}[97]:=\mathrm{r} 2=$ RegComplement [

ToRegex [RegularExpression [" $(\mathrm{a} \mid \mathrm{b}) *(\mathrm{aabb})(\mathrm{a} \mid \mathrm{b}) * "]]$,

$\{" a "$, "b" $\}$;

In [98]:= r3 = RegComplement [

ToRegex [RegularExpression [" (a $\mid \mathrm{b}) *(\mathrm{bbaa})(\mathrm{a} \mid \mathrm{b}) * "]]$,

$\{" a "$, "b" $\}$;

We compute the generating function $\sum_{n \geq 0} b_{n} z^{n}$, represented by $\mathrm{f} 2$, by taking the intersection of $r 2$ and $r 3$.

In $[99]:=f 2=$ GeneratingFunction [

RegIntersection $[r 2, r 3],\{n a "->z, " b "->z\}]$

// FullSimplify

Out [99] $=\left(1+z^{\wedge} 2+z^{\wedge} 3\right) /\left(1+z\left(-2+z-z^{\wedge} 2\right)\right)$

Now, we can conclude by showing that $f 2-2 * z * f 1$ is a constant. 
In[100]:= f2 - 2 z*f1// FullSimplify

Out $[100]=1$

\subsection{Miscellaneous problems}

Problem 12. A word $w$ avoids a subword pattern $p$ if and only if no contiguous subword (a.k.a factor) of $w$ is equal to $p$. Let $f_{k}(n)$ be the number of $n$-words over the alphabet $\{0,1\}$ that avoid the subword pattern $1^{k}=11 \cdots 1$. Find $F_{k}(z)=\sum_{n \geq 0} f_{k}(n) z^{n}$.

Solution: This is a generalization of Problem 2, which is the case $k=3$. We cannot define automata with parameters such as $k$, but we can use a recurrence relation to solve this problem instead. An $n$-word $w \in\{0,1\}^{*}$ avoids $1^{k}$ if and only if $w=1^{j} 0 w^{\prime}$, where $0 \leq j<k$ and $w^{\prime}$ is a binary $(n-j-1)$-word that avoids $1^{k}$. If we define $f(n)=0$ for all $n<0$, this leads to a recurrence relation for $f_{k}(n)$ :

$$
f_{k}(n)=\sum_{i=1}^{k} f_{k}(n-i)+[n<k], \quad \text { for all } n \in \mathbb{Z}
$$

The $[n<k]$ term accounts for the words $1^{n}$, where $n<k$.

Now we write this equation out in Mathematica using $\mathrm{f}[\mathrm{n}]$ to represent $f(n)$, and apply the Genfunlib-enhanced GeneratingFunction command to perform the generating function transform to both sides.

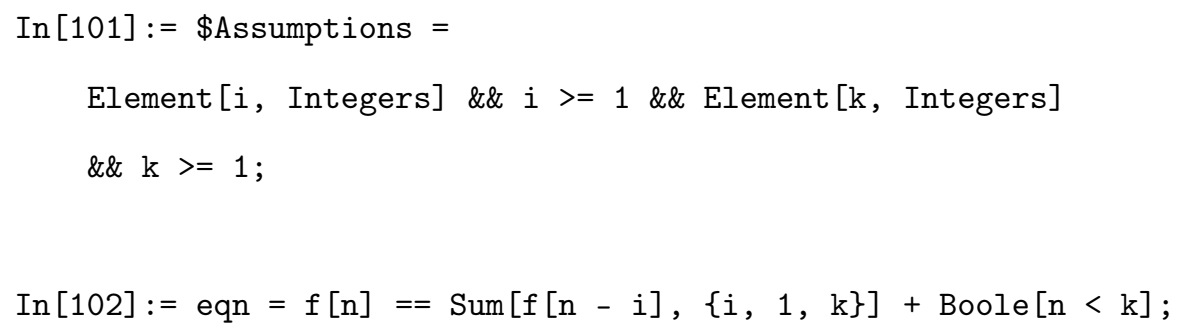




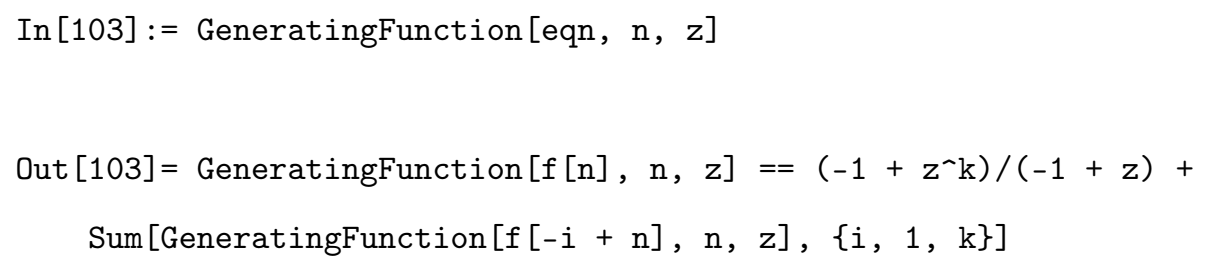

For some reason, this output must be evaluated again to get the desired result.

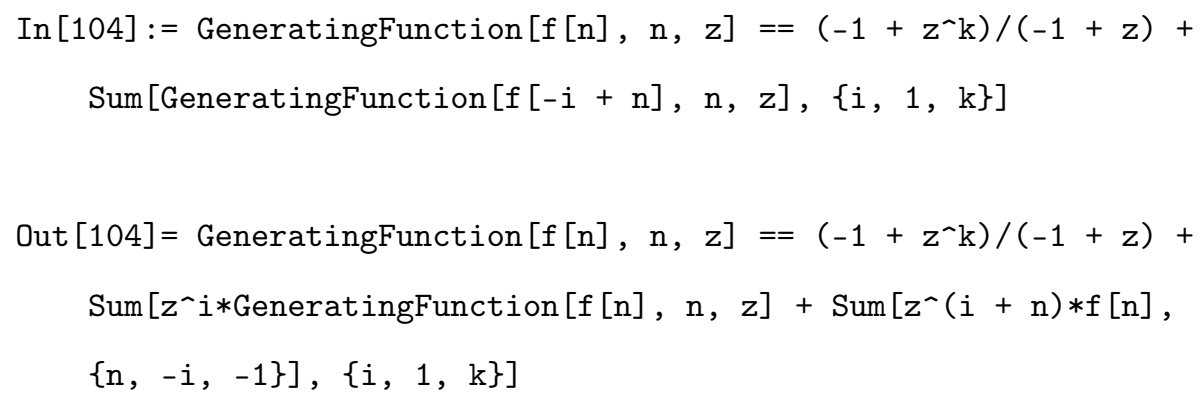

We can replace GeneratingFunction $[f[n], n, z]$, which represents $F(z)$, with the shorter expression $f f[z]$; and we can remove remaining $f[n]$ terms with zero, because they are all equal to zero.

$\operatorname{In}[105]:=\% / .\{$ GeneratingFunction $[f[n], n, z]->f f[z], f[n]->0\}$

$\operatorname{Out}[105]=\operatorname{ff}[z]==\left(-1+z^{\wedge} k\right) /(-1+z)+\left(z\left(-1+z^{\wedge} k\right) f f[z]\right) /(-1+z)$

In mathematical notation, this is

$$
F(z)=\frac{z F(z)\left(z^{k}-1\right)}{z-1}+\frac{z^{k}-1}{z-1}
$$

All that remains is to solve this linear equation.

$\operatorname{In}[106]:=f f[z] /$ Solve $[\%, f f[z]] / /$ First

$\operatorname{Out}[106]=\left(1-z^{\wedge} k\right) /\left(1-2 z+z^{\wedge}(1+k)\right)$ 
So the final answer is

$$
F(z)=\frac{1-z^{k}}{z^{k+1}-2 z+1} .
$$

Problem 13. Let $f(n)$ be the number of $n$-words without neighboring zeros from the alphabet $\{0,1,2\}$. Find an asymptotic expression for $f(n)$ as $n$ goes to infinity.

Solution: This $f(n)$ is the same as the one from Problem 8, where we discovered that the ordinary generating function for $f(n)$ is

$$
\sum_{n \geq 0} f(n) z^{n}=\frac{1+z}{1-2 z-2 z^{2}}
$$

The required asymptotic expression is extracted by simply applying Genfunlib's CoefLimit command to the generating function.

$\operatorname{In}[107]:=\operatorname{CoefLimit}\left[(1+z) /\left(1-2 z-2 z^{\wedge} 2\right), z, n\right]$

$\operatorname{Out}[107]=((1 / 2(-1+\operatorname{Sqrt}[3])) \sim(-1-$

n) $(1+1 / 2(-1+\operatorname{Sqrt}[3]))) /(2+2(-1+\operatorname{Sqrt}[3]))$

After automatic simplification, this says

$$
f(n) \sim \frac{1}{3}(3+\sqrt{3}) 2^{n-1}(\sqrt{3}-1)^{-n-1}, \text { as } n \rightarrow \infty .
$$

Problem 14. Define the class of plane trees, where $\mathcal{Z}_{1}$ marks nodes and $\mathcal{Z}_{2}$ marks path length, by

$$
\mathcal{T}=\mathcal{Z}_{1} \times \operatorname{SEQ}\left(\mathcal{T} \circ\left(\mathcal{Z}_{1} \times \mathcal{Z}_{2}\right)\right)
$$


The path length of a tree is the sum of the distances of all nodes to the root.

1. Find an asymptotic expression for the number of plane trees with $n$ nodes, as $n$ goes to infinity.

2. Find the number of plane trees with 7 nodes and pathlength 8 .

3. Find an asymptotic expression for the average path length of a plane tree with $n$ nodes, as $n$ goes to infinity.

Solution: To solve part 1 , we remove $\mathcal{Z}_{2}$ from the specification and use Genfunlib's ToGFEqns command to get an equation in $T\left(z_{1}\right)$, which is represented by $t[z[1]]$.

$\operatorname{In}[108]:=\operatorname{spec}=\operatorname{Spec}[\{$

$\mathrm{t}==\operatorname{SMTimes}[\mathrm{ZClass}[1], \operatorname{SMSeq}[t]]$

\}, False];

$\operatorname{In}[109]:=$ treeEqn $=$ First $[$ ToGFEqns $[$ spec, $\mathrm{z}]]$

Out $[109]=t[z[1]]==z[1] /(1-t[z[1]])$

The returned equation (treeEqn), which is $t\left(z_{1}\right)=z_{1} /\left(1-t\left(z_{1}\right)\right)$, can be given to Genfunlib's TreeAsymptot command to compute an asymptotic expression for $\left[z_{1}^{n}\right] t\left(z_{1}\right)$.

In [110]:= TreeAsymptot [treeEqn, n]

$\operatorname{Out}[110]=4 \wedge n((1 / \mathrm{n}) \wedge(3 / 2) /(4 \operatorname{Sqrt}[\mathrm{Pi}])+0[1 / \mathrm{n}] \sim(5 / 2))$

Converted to $\mathrm{AT}_{\mathrm{E}} \mathrm{X}$, this says that the number of plane trees with $n$ nodes is

$$
\left[z_{1}^{n}\right] T\left(z_{1}\right)=4^{n}\left(\frac{\left(\frac{1}{n}\right)^{3 / 2}}{4 \sqrt{\pi}}+O\left(n^{-5 / 2}\right)\right)
$$


Now, we assign the full specification, including $\mathcal{Z}_{2}$, to the augSpec variable, and save the corresponding bivariate ordinary generating function equation in the eqn variable.

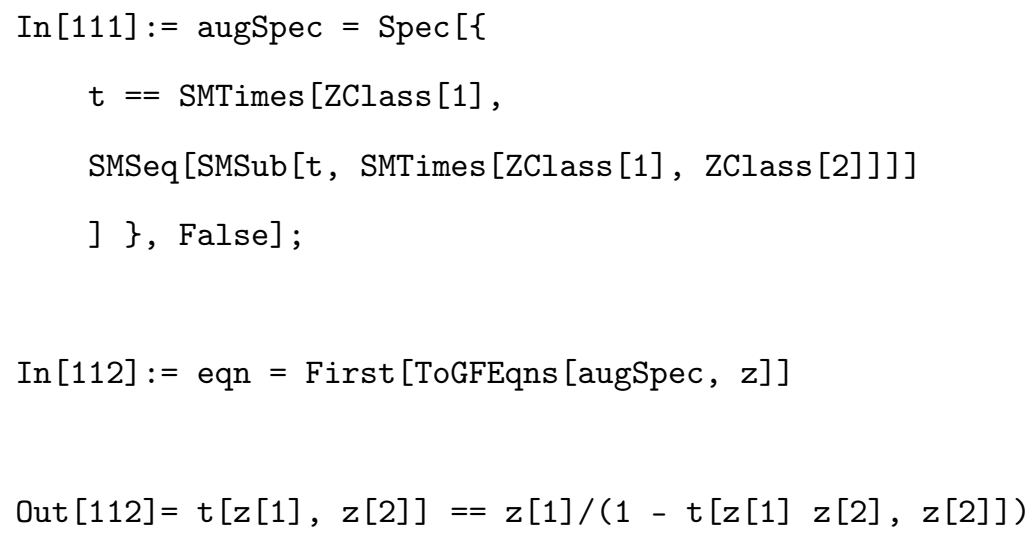

That is, we have

$$
T\left(z_{1}, z_{2}\right)=\frac{z_{1}}{1-T\left(z_{1} z_{2}, z_{2}\right)} .
$$

Equation (2) is all we need to answer part 2, since we can pass it to Genfunlib's CoefsByDerivs command to compute the initial terms of $t[z[1], z[2]]$.



This says $\left[z_{1}^{7} z_{2}^{8}\right] T\left(z_{1}, z_{2}\right)=10$. 
For part 3, we need to compute (an asymptotic estimate of)

$$
\frac{\left[z_{1}^{n}\right] T\left(z_{1}, 1\right)}{\left.\left[z_{1}^{n}\right] \frac{\partial}{\partial z_{2}} T\left(z_{1}, z_{2}\right)\right|_{z_{2}=1}} .
$$

We set $z_{2}$ to 1 on both sides of Equation (2), and save the result in eqn1. Then we solve that equation for $T\left(z_{1}, 1\right)$ and store the solution as to0.

$\operatorname{In}[114]:=$ eqn $1=$ eqn $/ . \mathbf{z}[2]->1$;

$\operatorname{In}[115]:=\operatorname{to0}=\operatorname{First}[t[z[1], 1] /$. Solve[eqn1, $t[z[1], 1]]]$

Out $[115]=1 / 2(1-\operatorname{Sqrt}[1-4 \mathrm{z}[1]])$

We define eqn2 as the equation obtained by differentiating both sides of Equation (2) with respect to $z_{2}$, and setting $z_{2}$ to 1 .

$\operatorname{In}[116]:=$ eqn2 $=\mathrm{D}[$ eqn, $z[2]] / . \mathrm{z}[2]->1$;

We define eqn3 as the equation obtained by differentiating both sides of Equation 2 with respect to $z_{1}$.

$\operatorname{In}[117]:=$ eqn3 $=\mathrm{D}[$ eqn $1, \mathrm{z}[1]] ;$

Finally, we define eqn4 as the equation obtained by eliminating $T\left(z_{1}, 1\right)$ and $\frac{\partial}{\partial z_{1}} T\left(z_{1}, 1\right)$ from the system eqn1 \&\& eqn2 \&\& eqn3.

$\operatorname{In}[118]:=$ eqn4 $=$ Eliminate [

eqn1 \&\& eqn2 \&\& eqn3, $\{t[z[1], 1]$,

Derivative $[1,0][t][z[1], 1]\}]$;

As eqn1 is for $T\left(z_{1}, 1\right)$, eqn4 is a single equation in which the only unknown is $\left.\frac{\partial}{\partial z_{2}} T\left(z_{1}, z_{2}\right)\right|_{z_{2}=1}$. We solve eqn4 for $\left.\frac{\partial}{\partial z_{2}} T\left(z_{1}, z_{2}\right)\right|_{z_{2}=1}$ and save the solution in t01. 


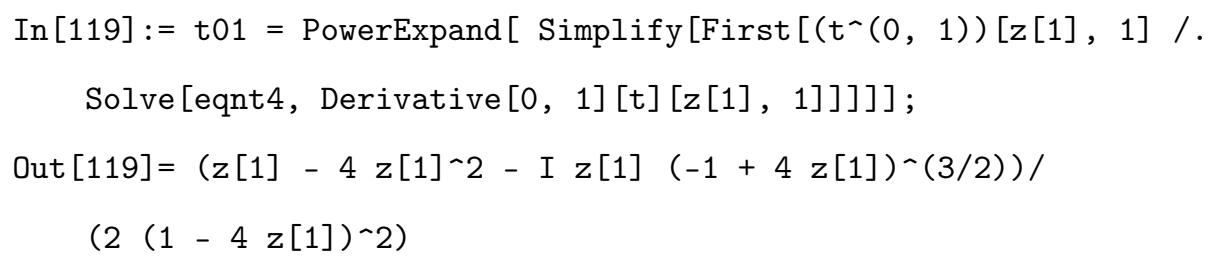

Now, Genfunlib does not have the ability to obtain an asymptotic expression for the coefficients of t00 or t01, but Mathematica does. We define the function coef Asympt which uses Mathematica's Series and SeriesCoefficient commands to extract an expression for coefficient $\mathrm{n}$ of a power series, and convert to an asymptotic form.

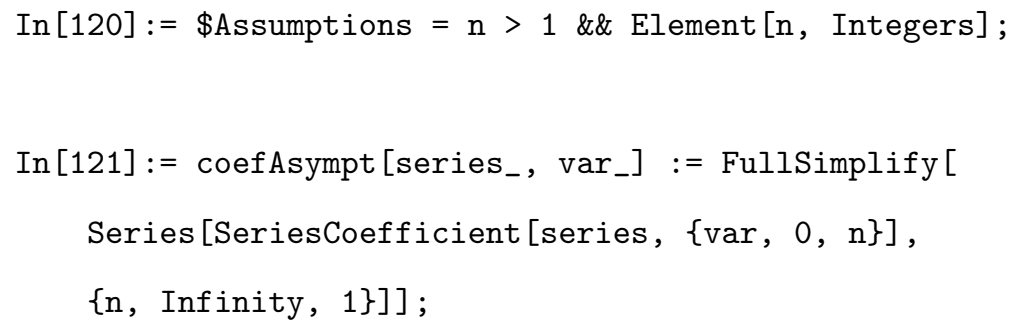

All that remains is to apply coef Asympt to t00 and t01 and divide, obtaining the answer to part 3 .

$\operatorname{In}[122]:=\operatorname{coefAsympt}[\mathrm{t} 01, \mathrm{z}[1]] / \operatorname{coef}$ Asympt $[\mathrm{t} 00, z[1]]$

$\operatorname{Out}[122]=1 / 2 \operatorname{Sqrt}[\mathrm{Pi}] \mathrm{n}^{\sim}(3 / 2)-\mathrm{n} / 2+1 / \operatorname{Sqrt}[0[1 / \mathrm{n}]]$

That is, as $n$ goes to infinity,

$$
\frac{\left[z_{1}^{n}\right] T\left(z_{1}, 1\right)}{\left.\left[z_{1}^{n}\right] \frac{\partial}{\partial z_{2}} T\left(z_{1}, z_{2}\right)\right|_{z_{2}=1}}=\frac{1}{2} \sqrt{\pi} n^{3 / 2}-\frac{n}{2}+O(\sqrt{n}) .
$$




\section{Further work}

In terms of further work, there is a number of possible directions. As mentioned previously, Mathematica itself causes difficulties when one wants to work with general big-O expressions, iterated sums with a symbolic number of dummy variables, and recurrence relations with polynomial coefficients. To get around these issues, perhaps a package could be written to provide alternatives or enhancements to the core Mathematica commands involved. We also mentioned that performace in Genfunlib should be improved across the board, and specifically in the area of regular languages. Multi-dimensional Newton iteration for computing coefficients from transcendental power series equations could be added.

Many counting problems involve not regular languages but context-free languages. Context-free languages do not have all the decidable properties that regular languages do, e.g. testing whether a context-free grammar is not decidable, and neither is testing whether a context-free grammar has an equivalent deterministic context-free grammar. However, if grammars are simply assumed to be unambiguous, something could be built, similar to Genfunlib's symbolic method functionality, to obtain generating functions that count the number of words of length $n$ in a context-free language.

Genfunlib focuses on counting combinatorial objects, but there are also the problems of enumeration and random generation. For families of objects represented by combinatorial species, a recent work by Pivoteau et. al [26] presents algorithms based on combinatorial Newton iteration [1] that are relevant to both of these problems. So far, the algorithm for random generation with species using Boltzmann sampling has not been implemented.

Extracting an asymptotic estimate for the coefficients $\left[z^{n}\right] F(z)$ of a power series is an interesting problem that Genfunlib approaches only on a relatively 
basic level. Algorithms that handle a larger set of power series $F(z)$ are needed.

In addition, algorithms and implementations for multivariate asymptotics would be useful. Some results in this area have been found [24, 28], and Raichev's amgf package for Sage implements a method for computing the asymptotic expansion of

$$
\left[z_{1}^{r \alpha_{1}} z_{2}^{r \alpha_{2}} \ldots\right] F\left(z_{1}, z_{2}, \ldots\right)
$$

as $r \rightarrow \infty$, for a certain class of multivariate power series $F\left(z_{1}, z_{2}, \ldots\right)$.

\section{References}

[1] F. Bergeron, G. Labelle, and P. Leroux. Combinatorial species and tree-like structures, volume 67 of Encyclopedia of Mathematics and its Applications. Cambridge University Press, Cambridge, 1998.

[2] A. Bostan, F. Chyzak, F. Ollivier, B. Salvy, É. Schost, and A. Sedoglavic. Fast computation of power series solutions of systems of differential equations. In Proceedings of the Eighteenth Annual ACM-SIAM Symposium on Discrete Algorithms, pages 1012-1021, New York, 2007. ACM.

[3] A. Bostan and E. Schost. A simple and fast algorithm for computing exponentials of power series. Inform. Process. Lett., 109(13):754-756, 2009.

[4] C. Brabrand and J. Thomsen. Typed and unambiguous pattern matching on strings using regular expressions. In Proceedings of the 12th international ACM SIGPLAN symposium on Principles and practice of declarative programming, pages 243-254. ACM, 2010.

[5] R. P. Brent and H. T. Kung. Fast algorithms for manipulating formal power series. J. Assoc. Comput. Mach., 25(4):581-595, 1978. 
[6] D. Cox, J. Little, and D. O'Shea. Ideals, varieties, and algorithms. Undergraduate Texts in Mathematics. Springer, New York, third edition, 2007.

[7] D. Dominici. Nested derivatives: a simple method for computing series expansions of inverse functions. Int. J. Math. Math. Sci., (58):3699-3715, 2003.

[8] P. Dumas and B. Salvy. Maple and the Putnam Competition. Rapport de recherche 179, INRIA, 1995.

[9] A. Engel. Problem-Solving Strategies. Problem Books in Mathematics. Springer-Verlag, New York, 1998.

[10] P. Flajolet, B. Salvy, and P. Zimmermann. Automatic average-case analysis of algorithms. Theoret. Comput. Sci., 79(1, (Part A)):37-109, 1991.

[11] P. Flajolet and R. Sedgewick. Analytic Combinatorics. Cambridge University Press, Cambridge, 2009.

[12] T. Gowers, J. Barrow-Green, and I. Leader, editors. The Princeton Companion to Mathematics. Princeton University Press, Princeton, NJ, 2008.

[13] Y. Gurevich. What is an algorithm? In SOFSEM 2012: theory and practice of computer science, volume 7147 of Lecture Notes in Comput. Sci., pages 31-42. Springer, Heidelberg, 2012.

[14] T. Jiang and B. Ravikumar. Minimal NFA problems are hard. SIAM J. Comput., 22(6):1117-1141, 1993.

[15] D. Jurafsky, J. Martin, A. Kehler, K. Vander Linden, and N. Ward. Speech and Language Processing: An Introduction to Natural Language Processing, Computational Linguistics, and Speech Recognition, volume 2. MIT Press, 2000. 
[16] M. Kauers. Computer algebra and power series with positive coefficients. In Proceedings of FPSAC, volume 7, 2007.

[17] W. Koepf. Efficient computation of chebyshev polynomials in computer algebra. Computer Algebra Systems: A Practical Guide. John Wiley, Chichester, pages 79-99, 1999.

[18] W. Koepf. Efficient computation on truncated power series: direct approach versus Newton's method. Util. Math., 83:37-55, 2010.

[19] M. Li and P. Vitányi. An Introduction to Kolmogorov Complexity and its Applications. Texts in Computer Science. Springer, New York, third edition, 2008.

[20] A. MacFie. Software for enumerative and analytic combinatorics. Honours project, Carleton University, 2012.

[21] C. Mallinger. Algorithmic manipulations and transformations of univariate holonomic functions and sequences. Diploma thesis, RISC, J. Kepler University, Linz, 1996.

[22] A. Møller. dk.brics.automaton - finite-state automata and regular expressions for Java, 2010. http://www.brics.dk/automaton/.

[23] J. Moses. Algebraic simplification: A guide for the perplexed. Comm. ACM, 14:527-537, 1971.

[24] R. Pemantle and M. C. Wilson. Analytic Combinatorics in Several Variables, volume 140 of Cambridge Studies in Advanced Mathematics. Cambridge University Press, Cambridge, 2013.

[25] S. Pemmaraju and S. Skiena. Computational Discrete Mathematics. Cambridge University Press, Cambridge, 2003. 
[26] C. Pivoteau, B. Salvy, and M. Soria. Algorithms for combinatorial structures: well-founded systems and Newton iterations. J. Combin. Theory Ser. A, 119(8):1711-1773, 2012.

[27] H. Piwowar. Value all research products. Nature, 493:159, January 2013.

[28] A. Raichev and M. C. Wilson. Asymptotics of coefficients of multivariate generating functions: improvements for multiple points. Online J. Anal. Comb., (6):21, 2011.

[29] Wolfram Research. Wolfram Mathematica 9 Documentation Center. http: //reference.wolfram.com/mathematica/guide/Mathematica.html, 2014. [Online; accessed 2014-02-20].

[30] R. Rusczyk. Are You Ready for Intermediate Counting \& Probability? http://data.artof problemsolving.com/aops20/courses/ IntermCounting/PreTest.pdf, 2007. [Online; accessed 2013-12-15].

[31] R. Rusczyk. AoPS Classes Math Jam. http://www . artofproblemsolving.com/School/mathjams.php?mj_id=254, 2009. [Online; accessed 2013-12-08].

[32] H. Ruskeepää. Mathematica Navigator: Mathematics, Statistics, and Graphics. Gulf Professional Publishing, 2004.

[33] J. Shallit. What's the greatest innovation - Survey responses - Jeffrey O Shallit. http://www.spiked-online.com/index.php? /innovationsurvey/article/3278/, 2006. [Online; accessed 2013-11-15].

[34] J. Shallit. A Second Course in Formal Languages and Automata Theory, volume 179. Cambridge University Press, 2009.

[35] M. Sipser. Introduction to the Theory of Computation, volume 2. Thomson Course Technology Boston, 2006. 
[36] R. Stanley. Enumerative Combinatorics. Vol. 2, volume 62 of Cambridge Studies in Advanced Mathematics. Cambridge University Press, Cambridge, 1999.

[37] M. Trott. The Mathematica guidebook for programming. Springer, New York, 2004.

[38] J. von zur Gathen and J. Gerhard. Modern Computer Algebra. Cambridge University Press, Cambridge, second edition, 2003.

[39] H. Wilf. What is an answer? J. Amer. Math. Monthly, 89(5):289-292, 1982.

\section{Appendix}

\section{Asymptotics.m}

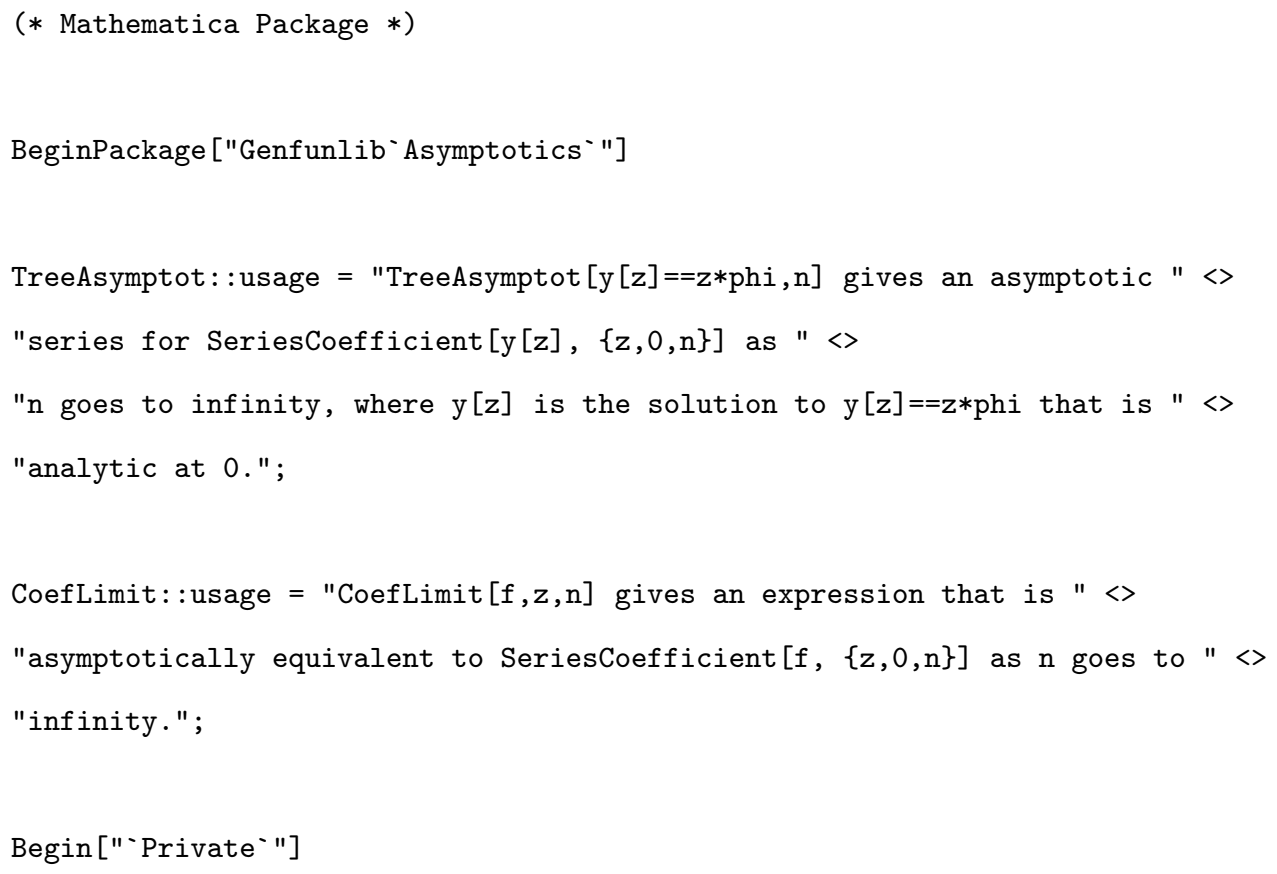




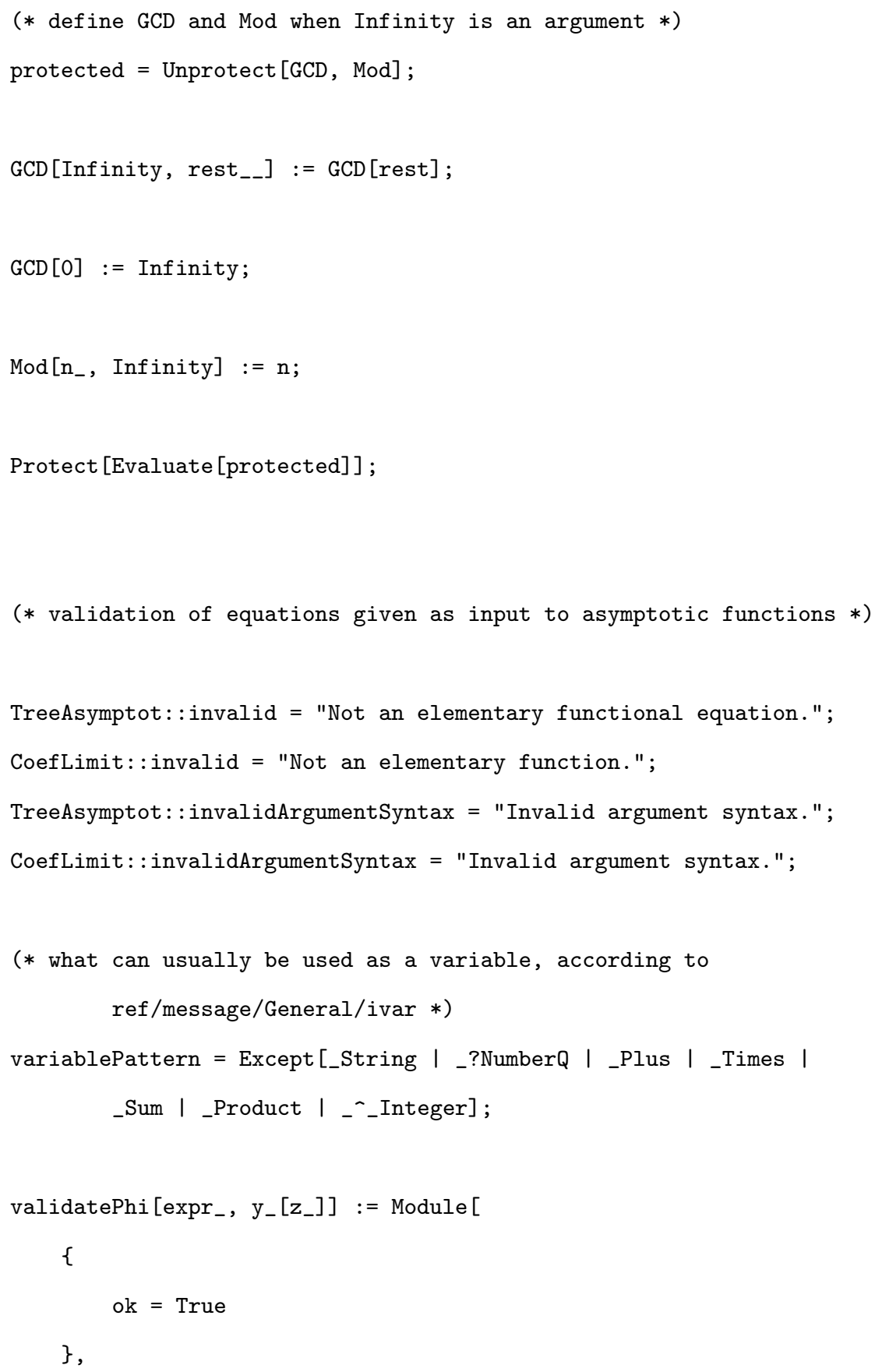




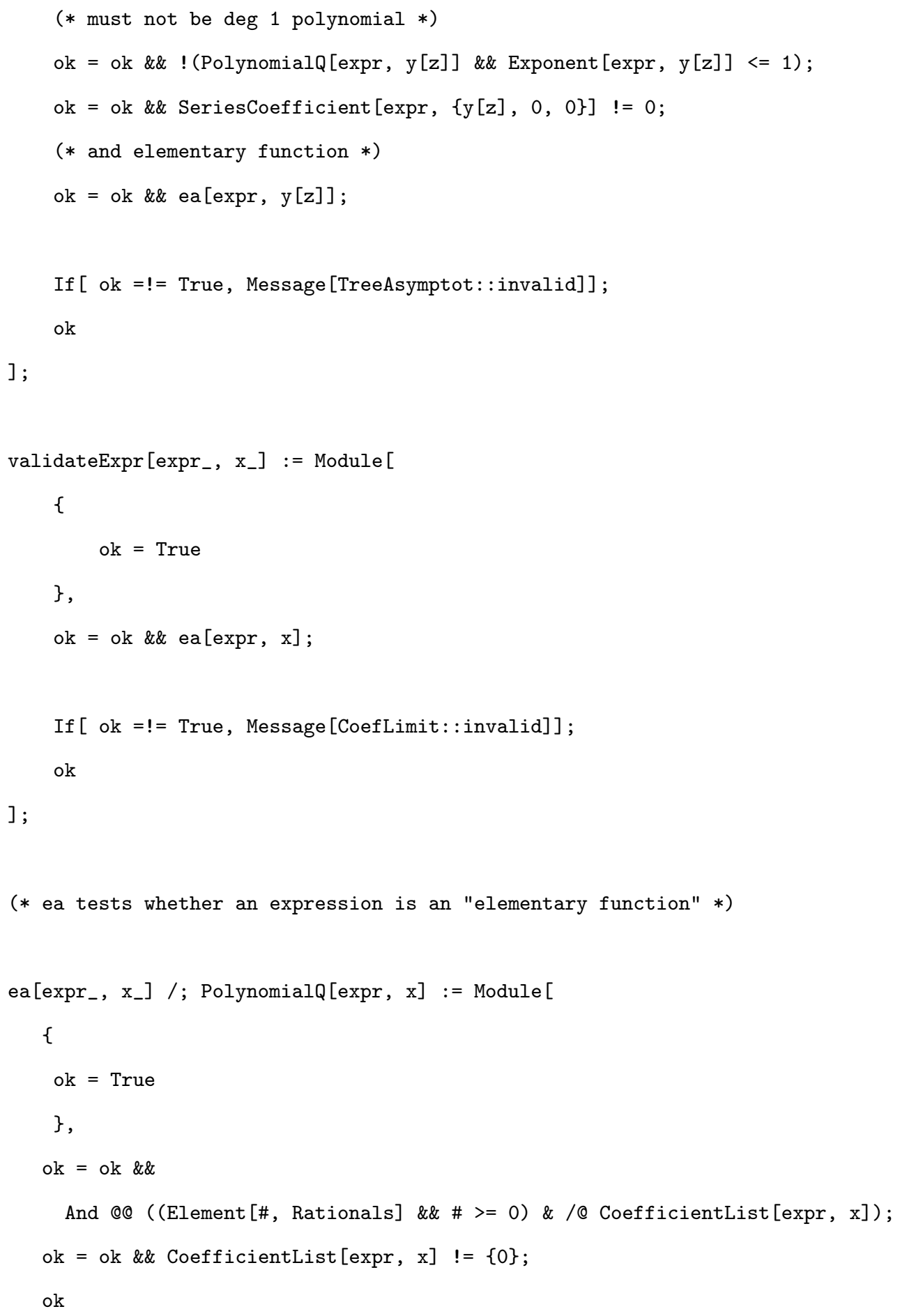


];

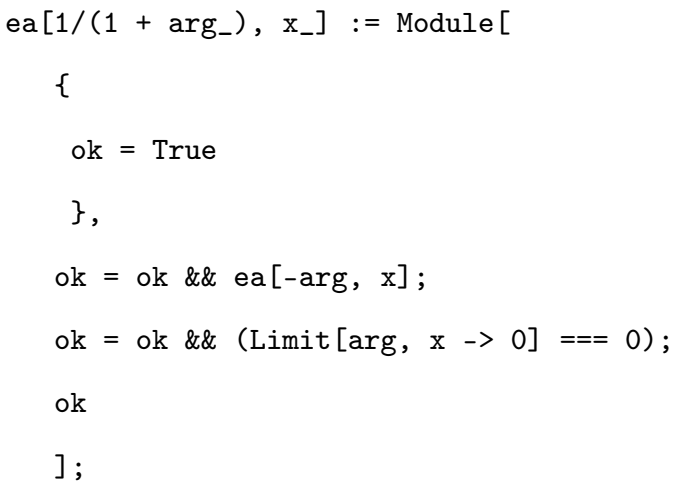




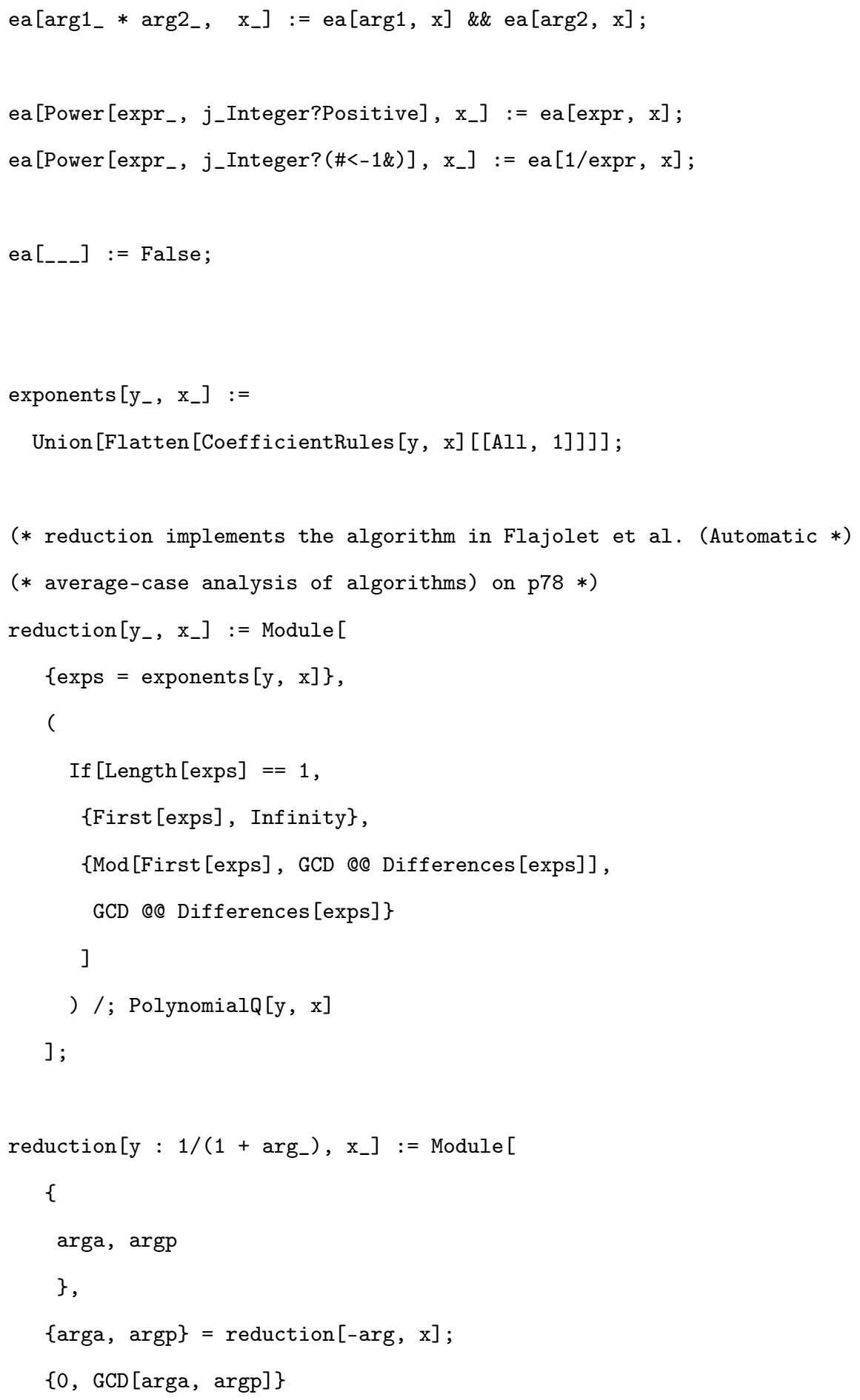


] ;

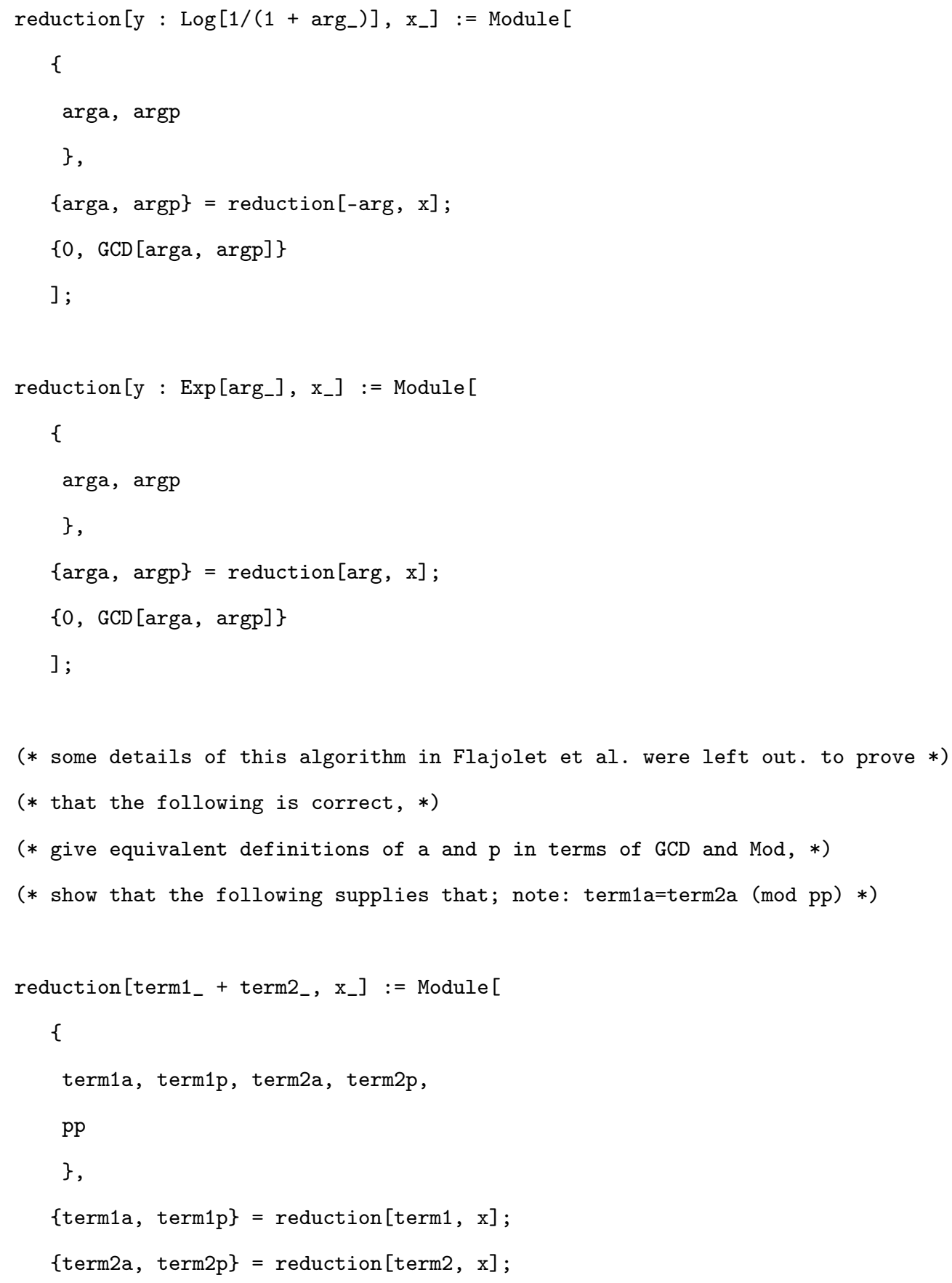




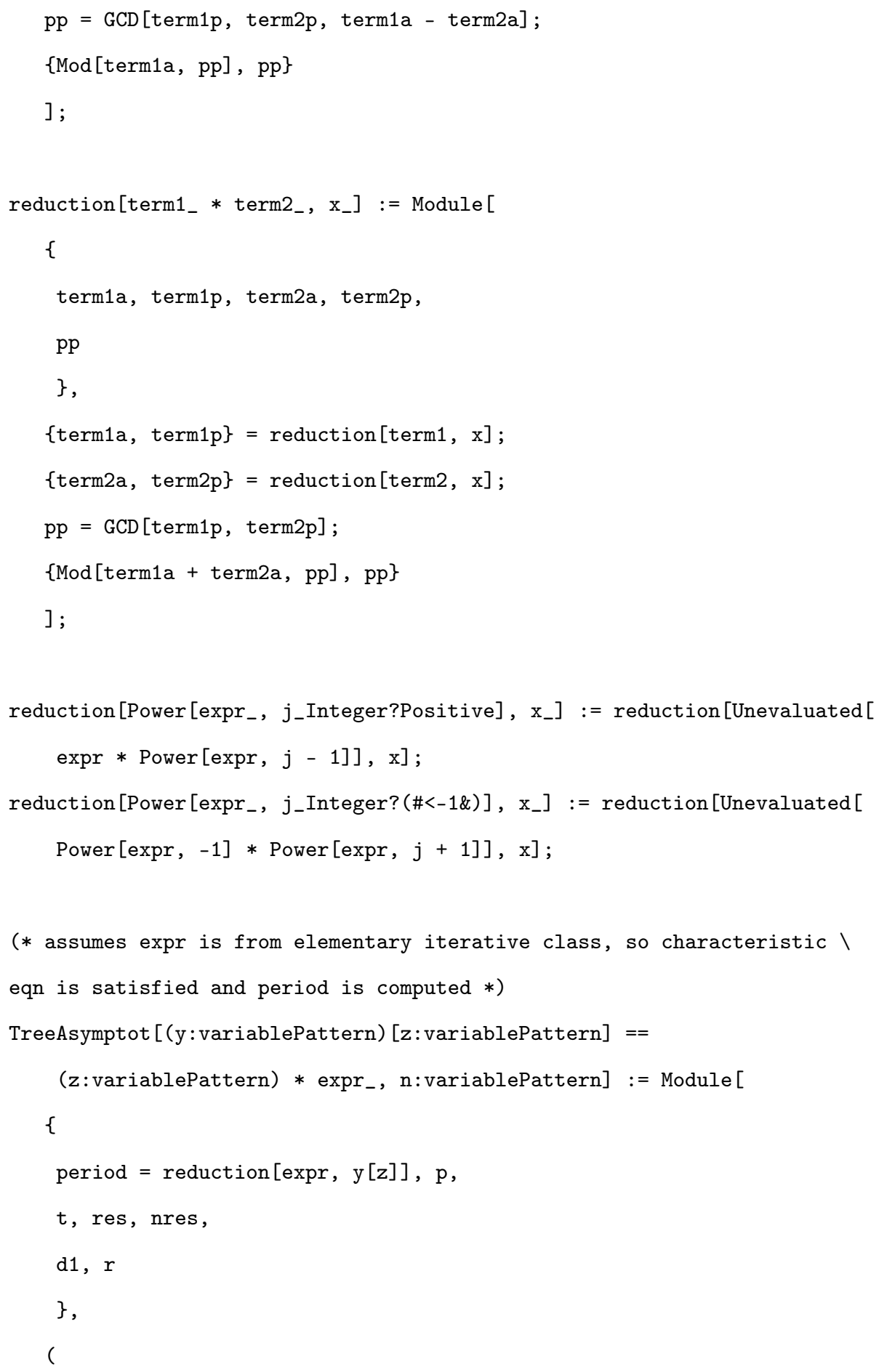




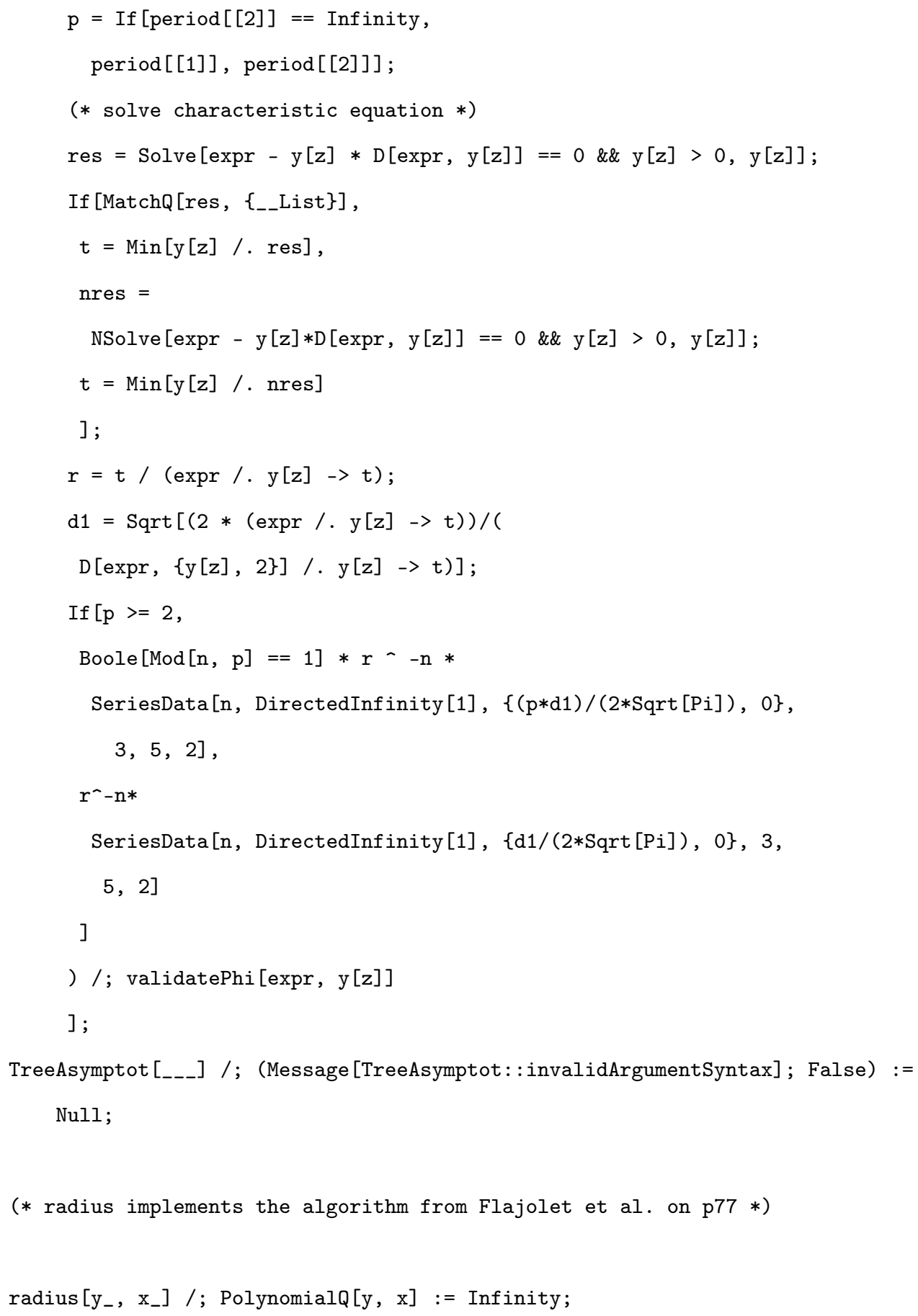




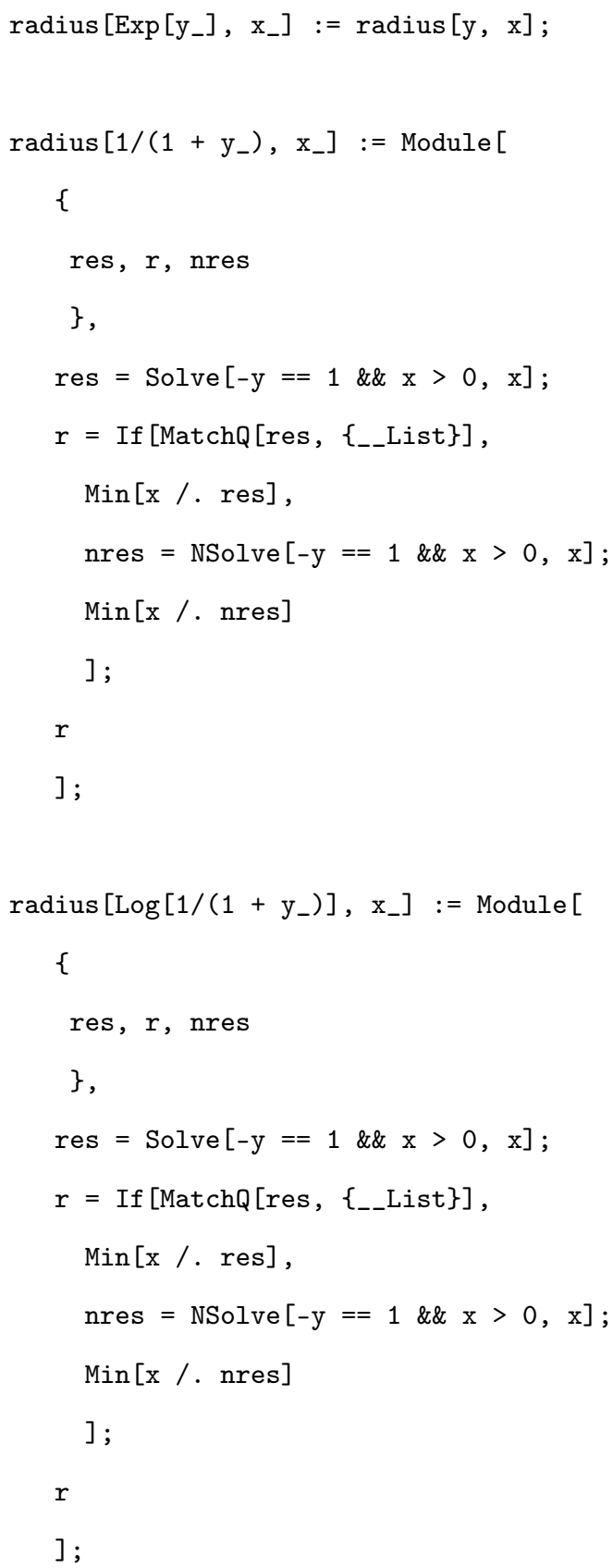




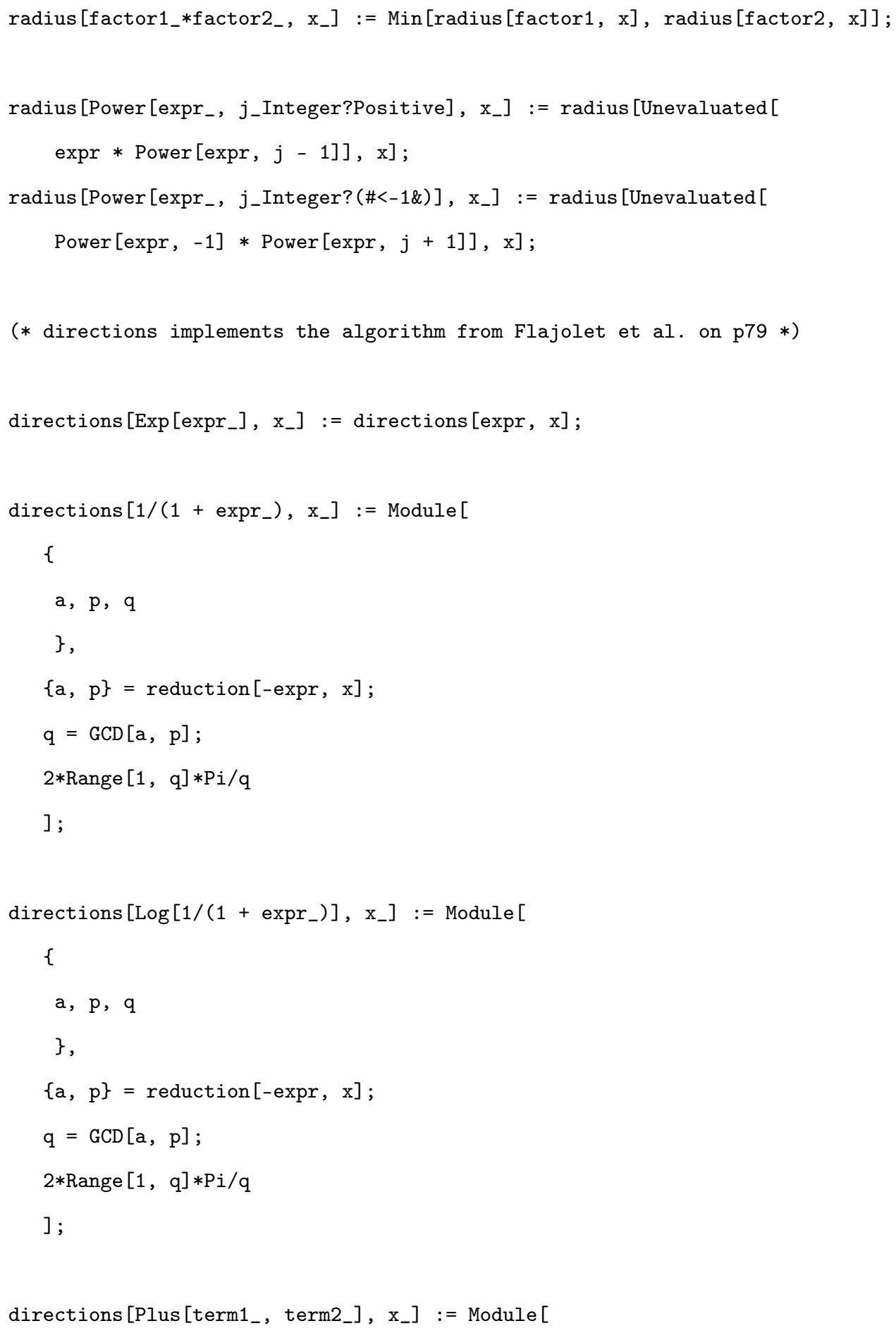




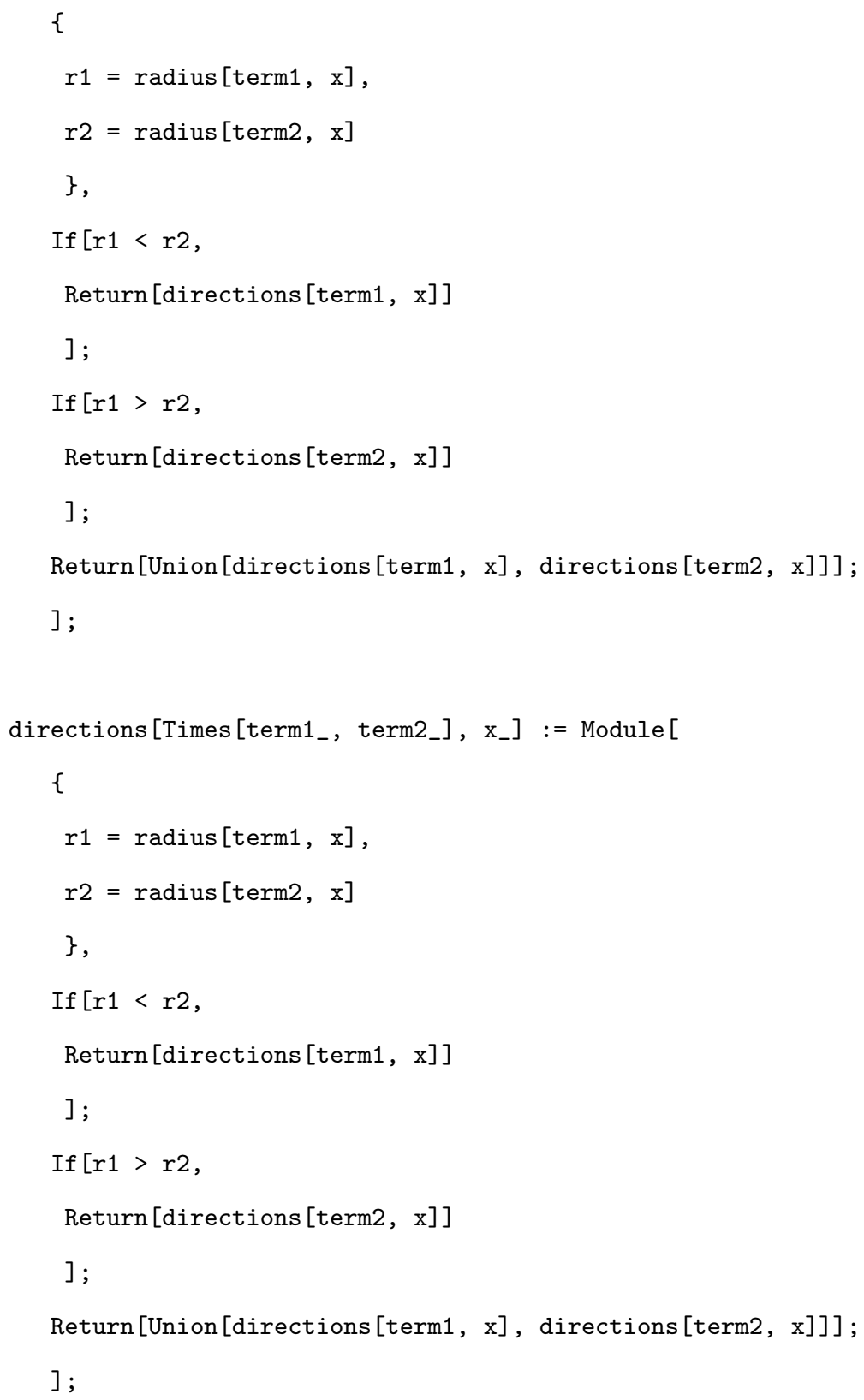




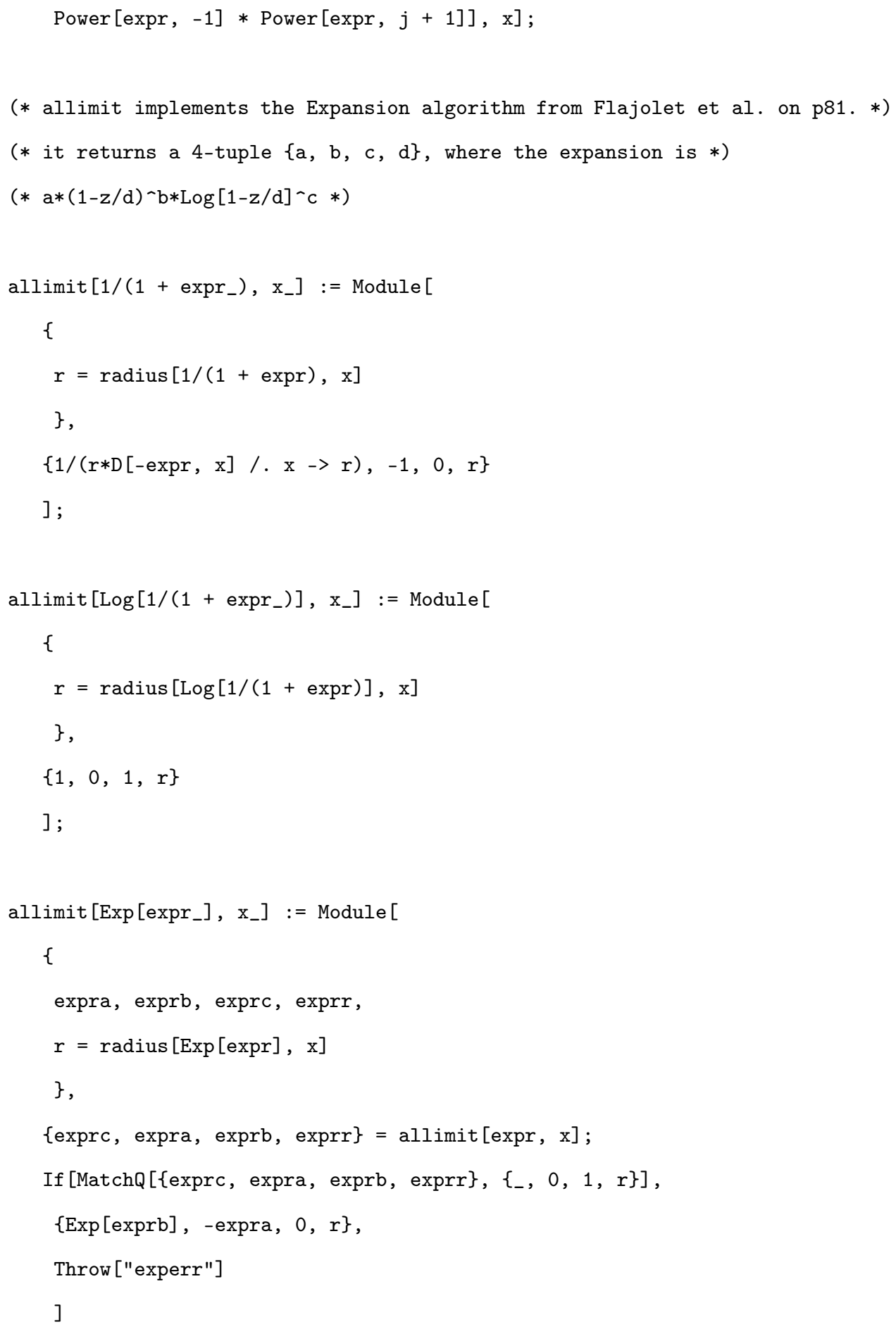


];

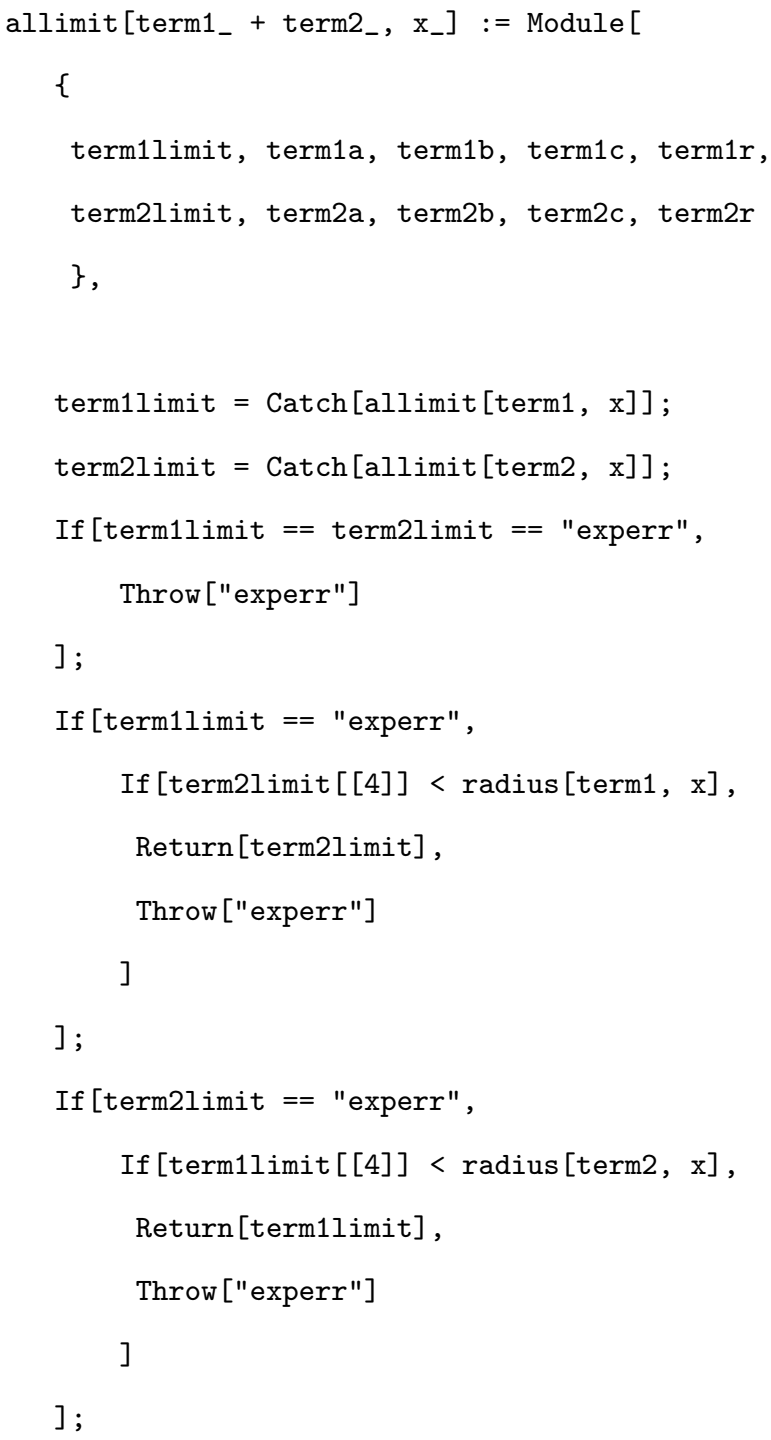




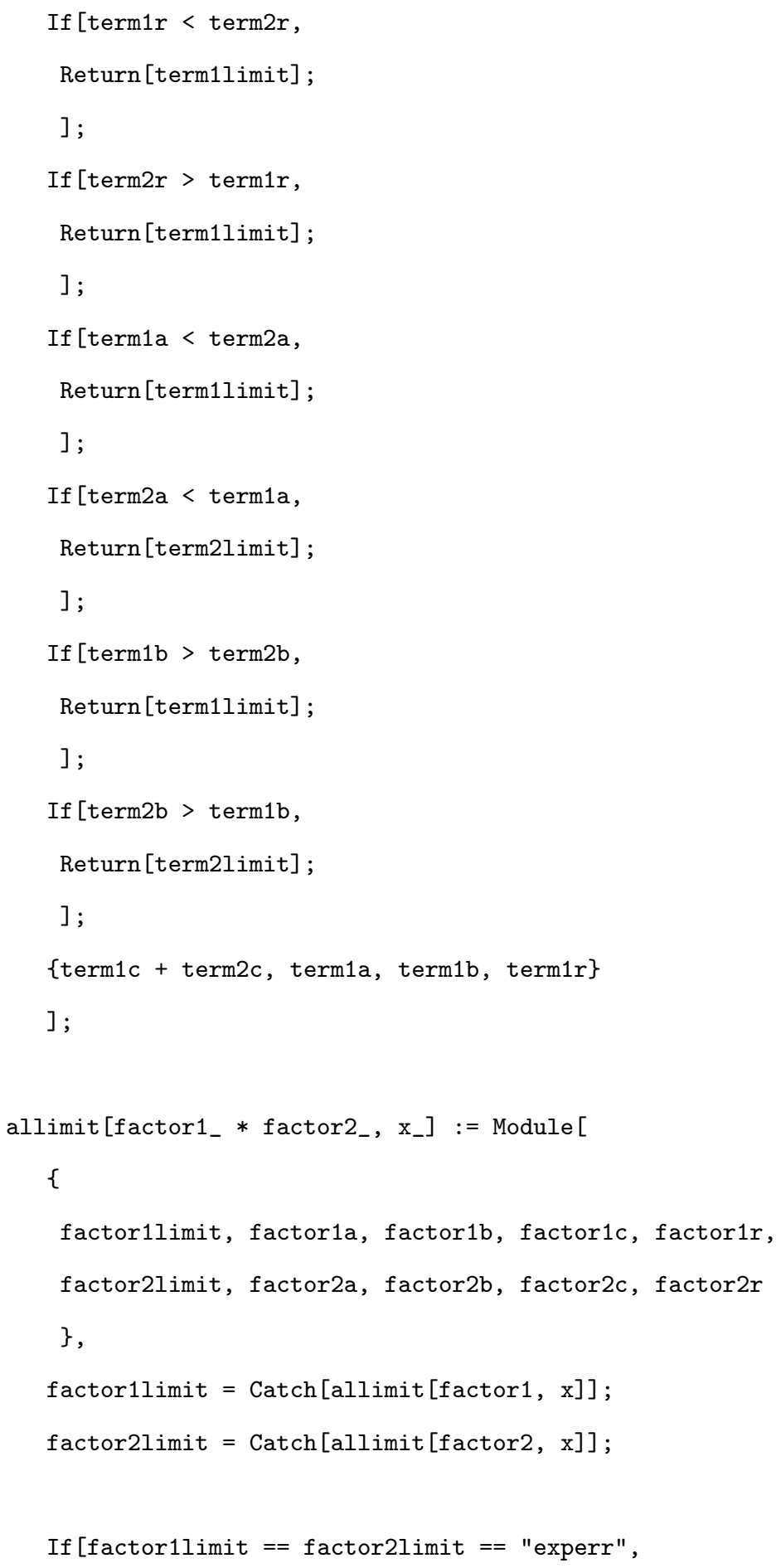




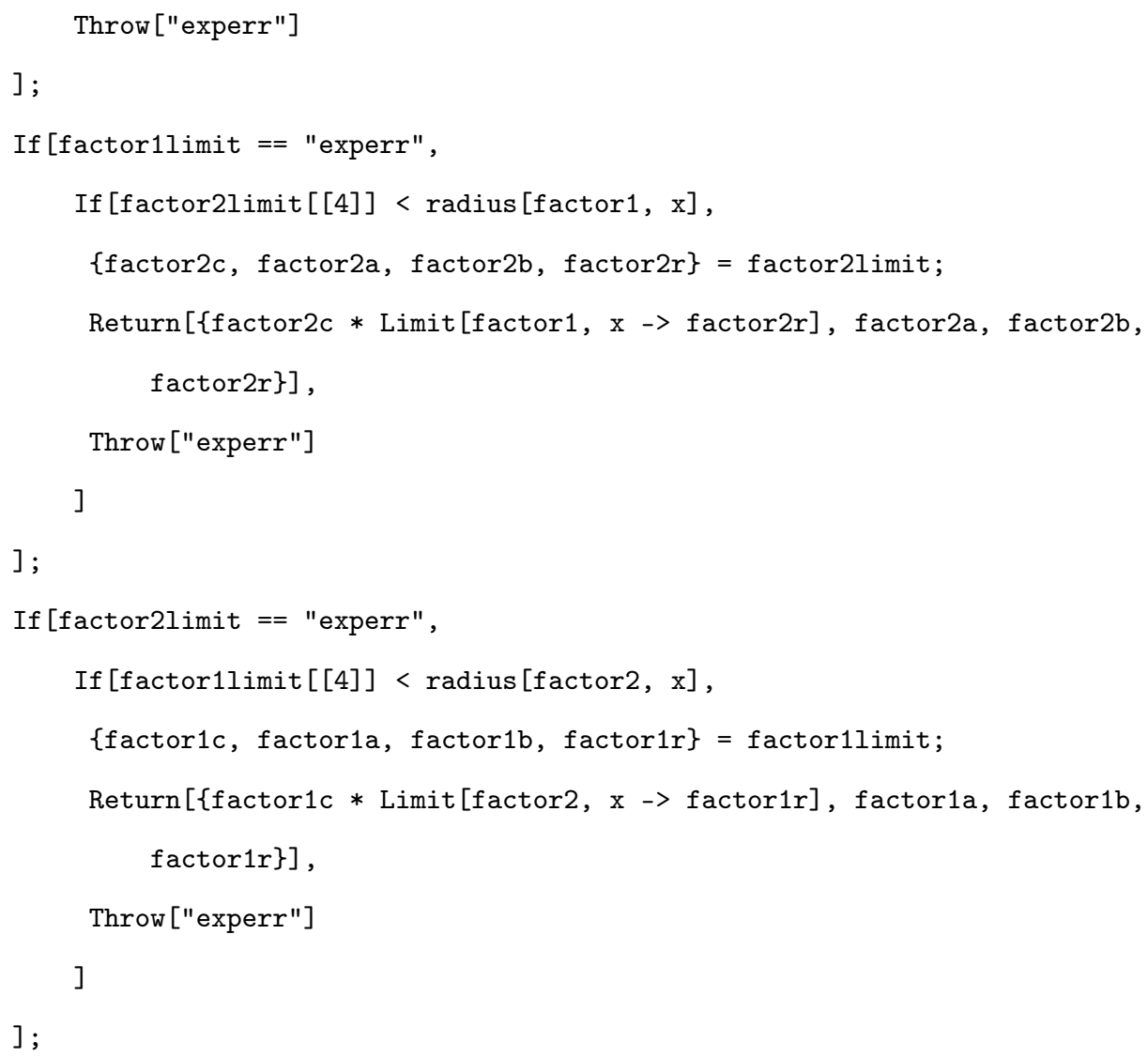




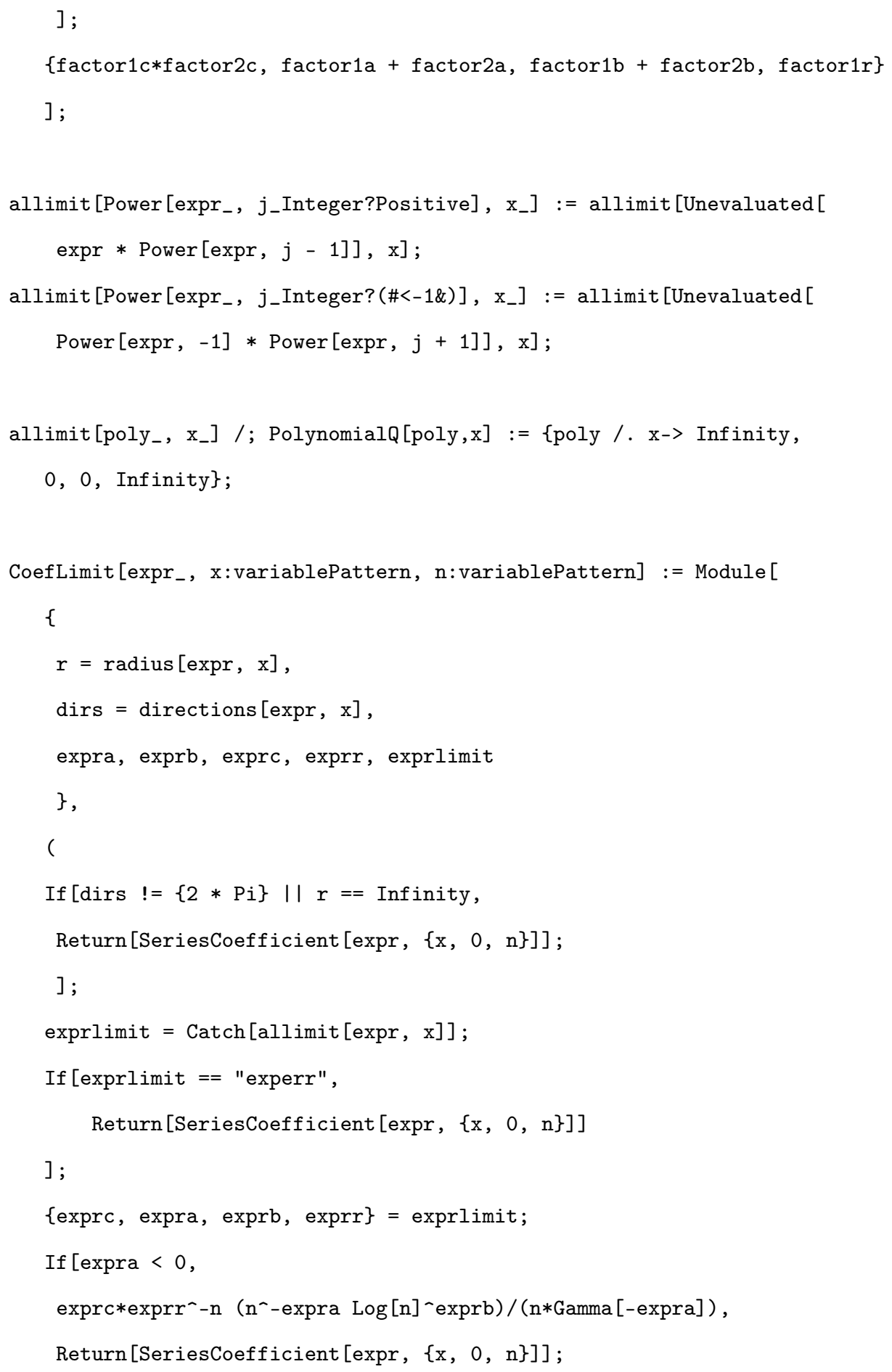






\section{GFeqn2rec.m}

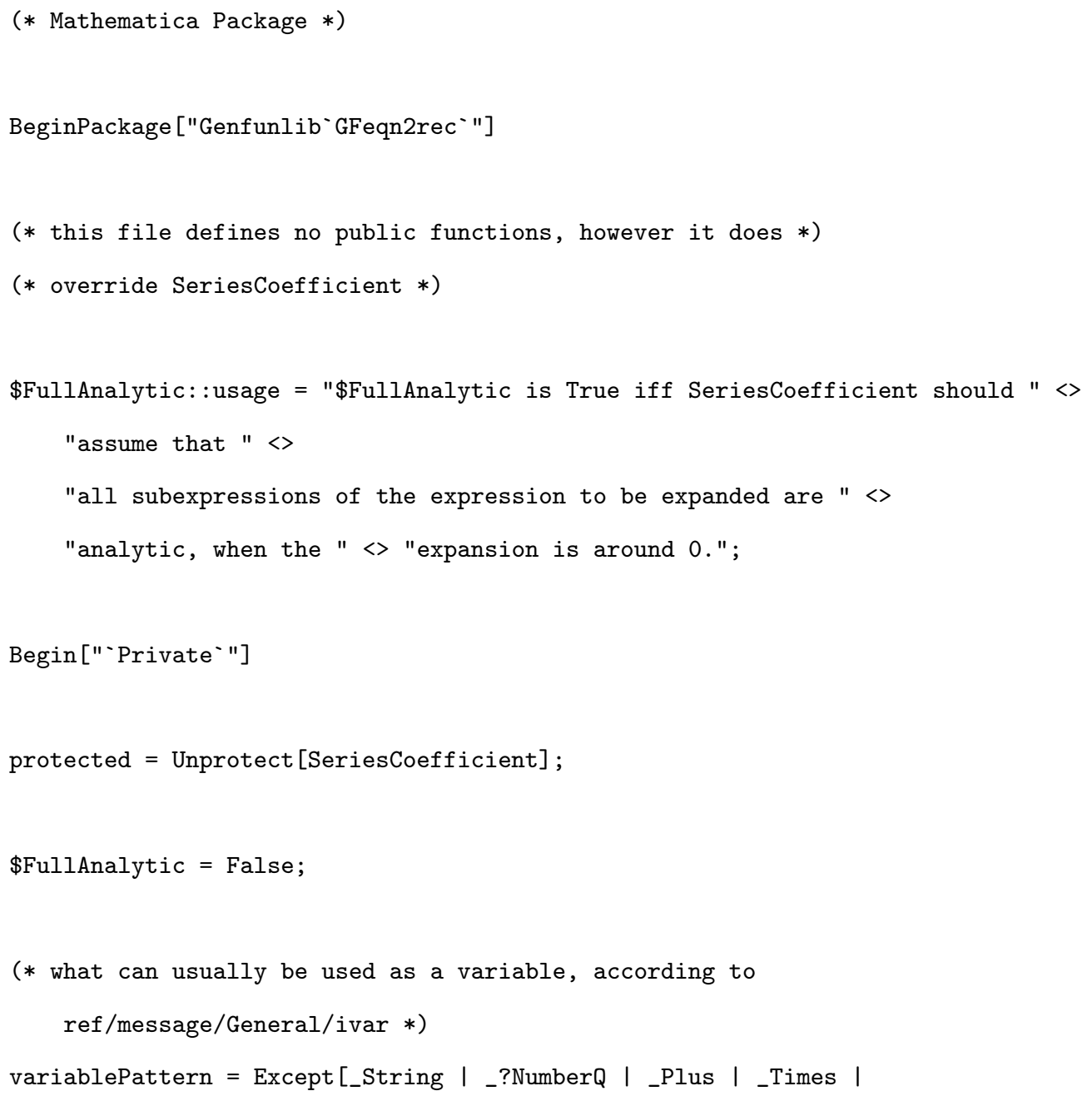




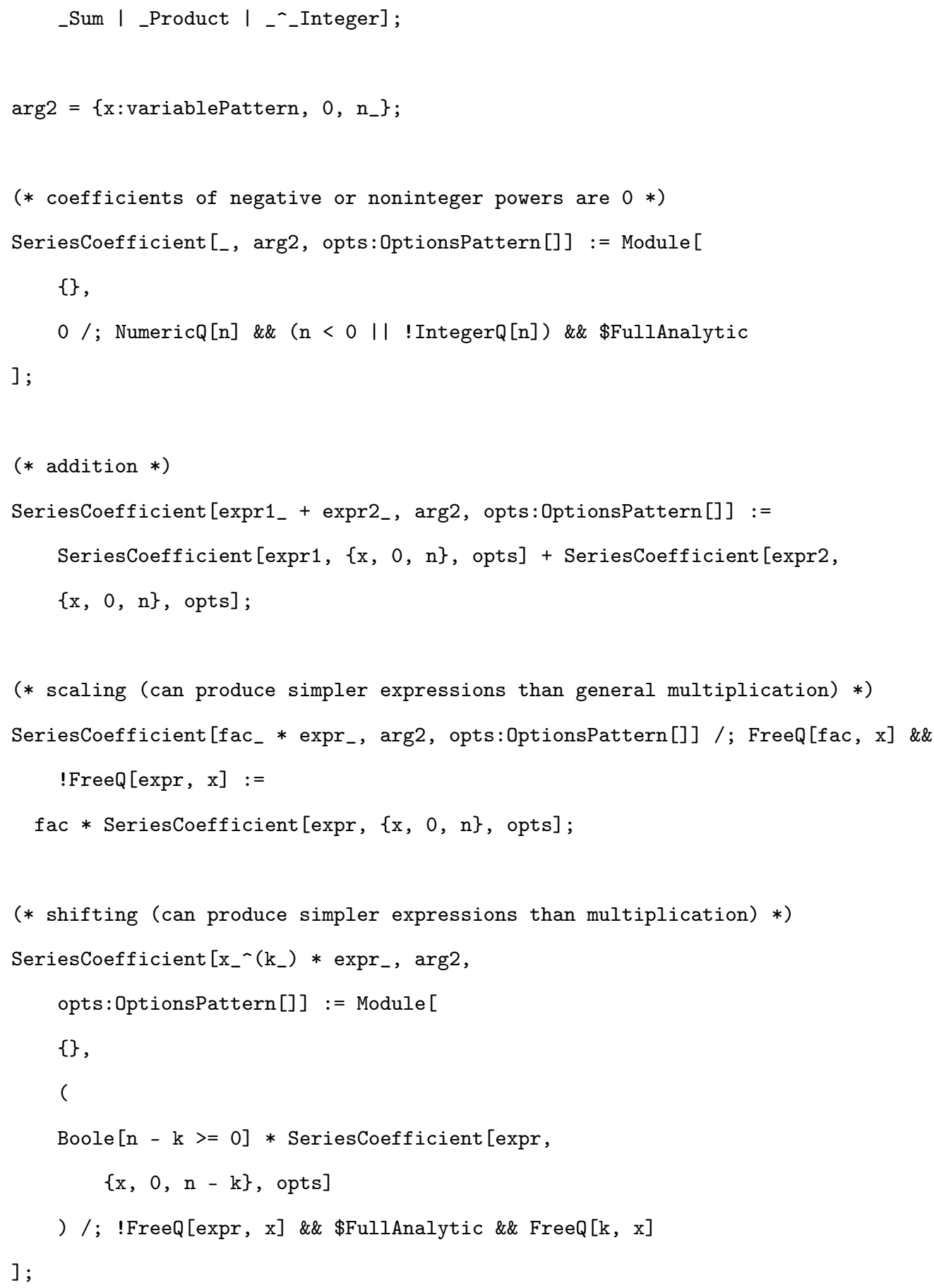




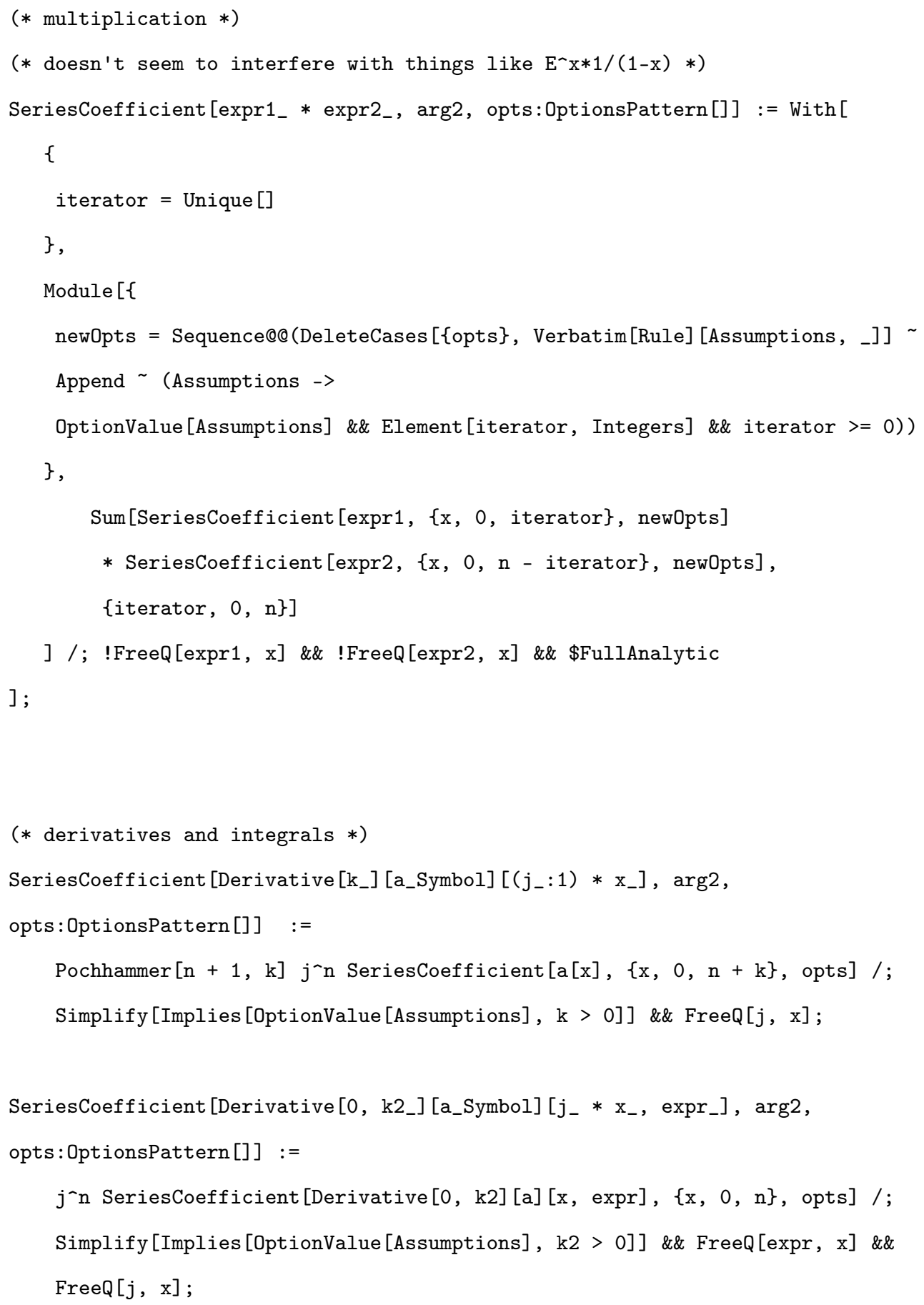




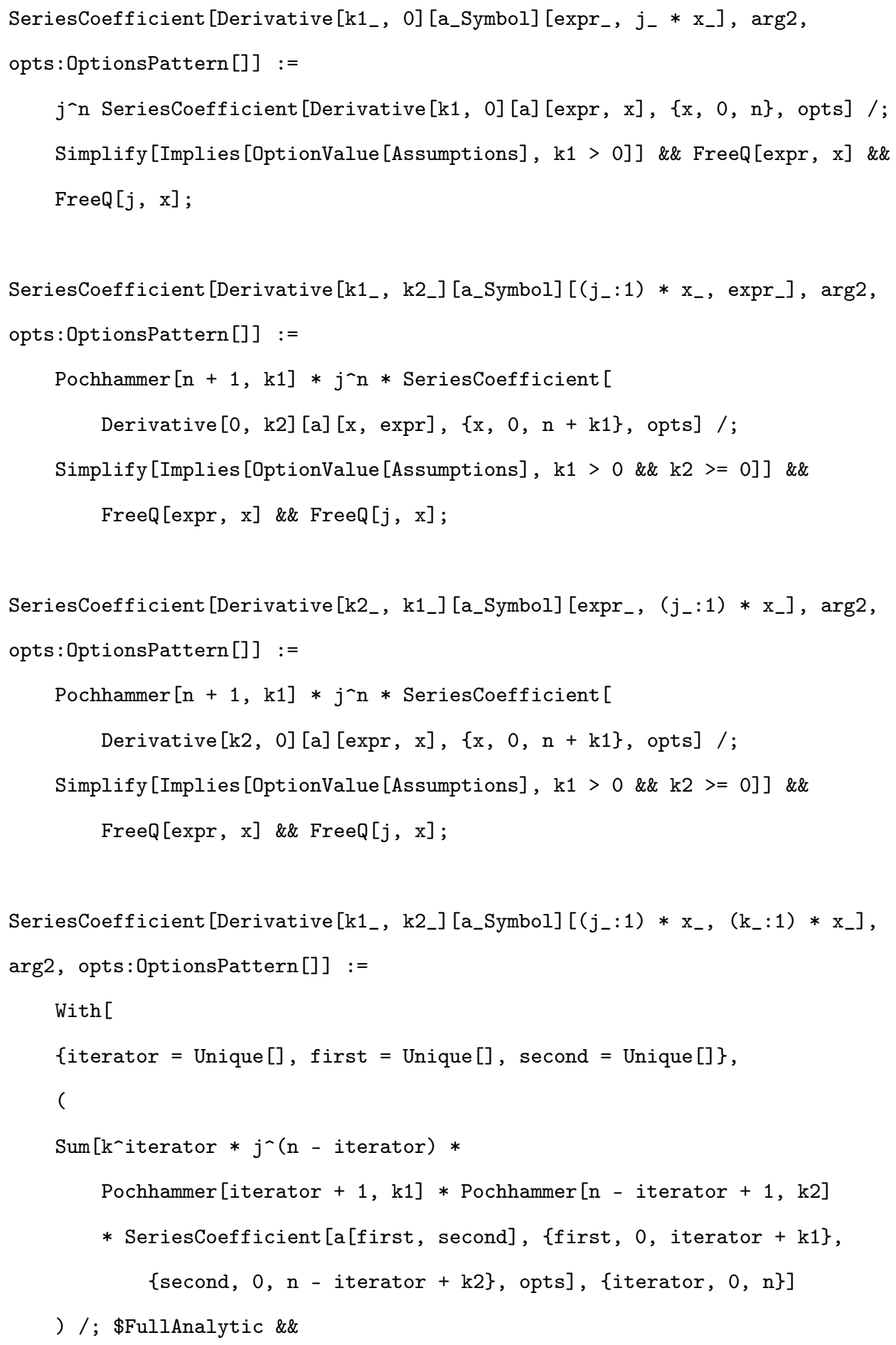




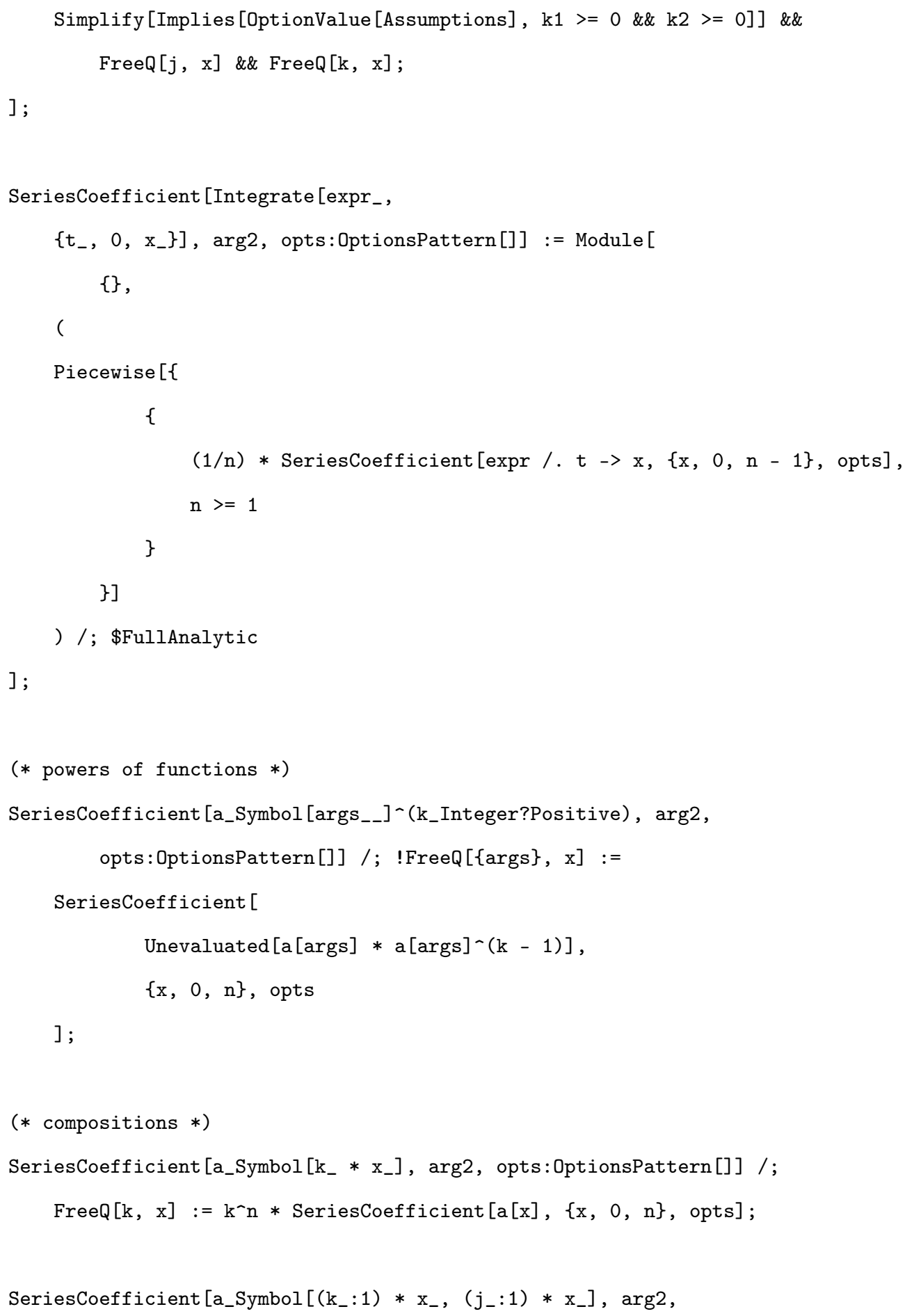









\section{RegularLanguages.m}

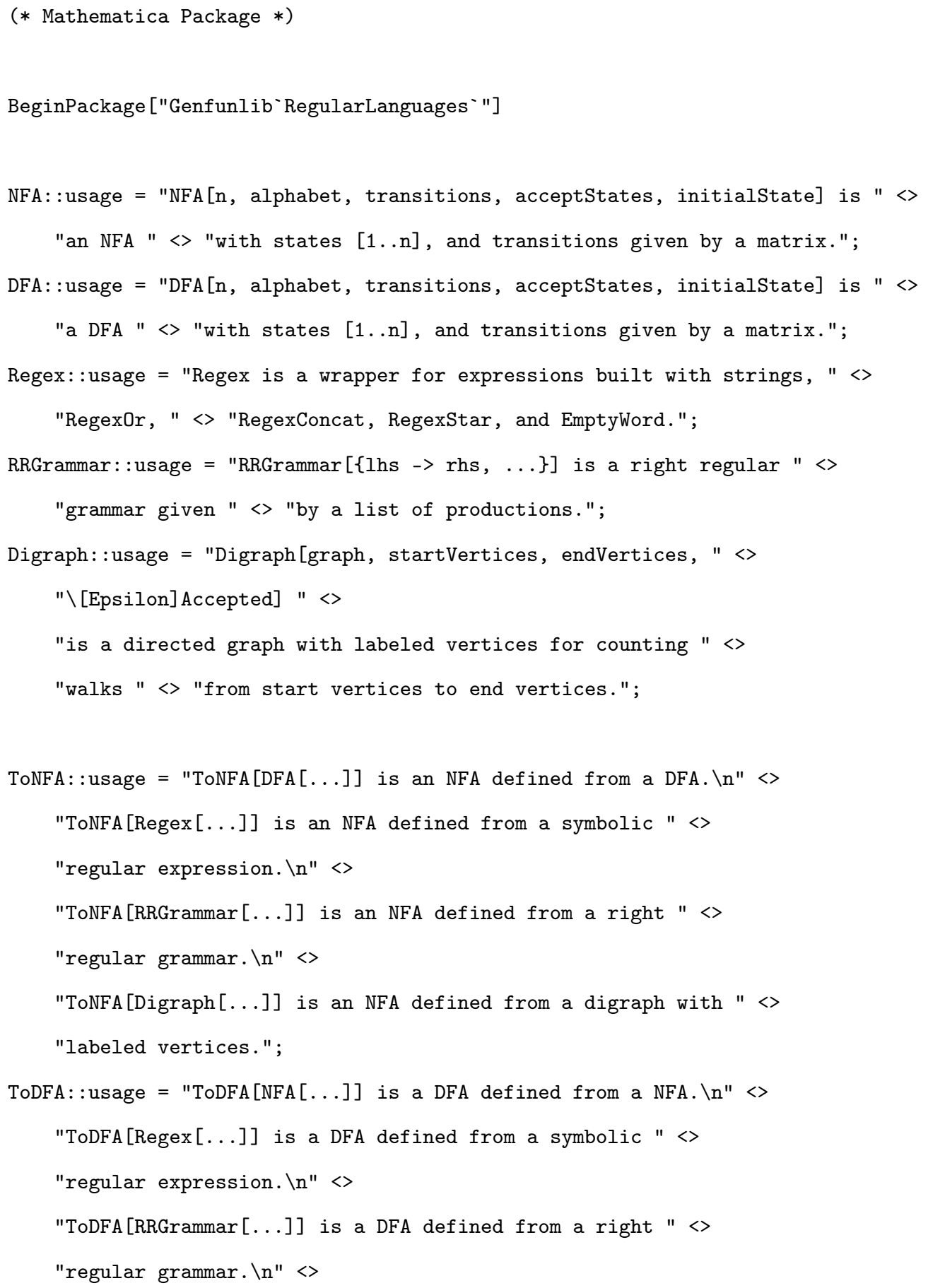


"ToDFA[Digraph[...]] is a DFA defined from a digraph with " <>

"labeled vertices.";

ToRegex: :usage $=$ "ToRegex $[\operatorname{NFA}[\ldots]]$ is a symbolic regular expression " <>

"defined from an NFA. \n" <>

"ToRegex[DFA[...]] is a symbolic regular expression defined from a DFA. \n" <>

"ToRegex[RegularExpression[\"regex\"]] is a symbolic regular expression " <>

"defined from a restricted Mathematica regular expression. \n" <>

"ToRegex[RRGrammar[...]] is a symbolic regular expression defined " <>

"from a right regular grammar. \n"

"ToRegex[Digraph[...]] is a symbolic regular expression defined from a " <>

"digraph with labeled vertices.";

ToRRGrammar: :usage = "ToRRGrammar[NFA[...]] is a right regular grammar " $<$

"defined from a NFA. In" <>

"ToRRGrammar[DFA[...]] is a right regular grammar defined from a DFA. \n" $<>$

"ToRRGrammar[Regex[...]] is a right regular grammar defined from a " $>$

"symbolic regular expression. \n" <>

"ToRRGrammar[Digraph[...]] is a right regular grammar defined from a " $<$

"digraph with labeled vertices.";

ToDigraph: :usage $=$ "ToDigraph[NFA[...]] is a directed graph defined " $>$

"from an NFA. \n" $<>$

"ToDigraph[DFA[...]] is a directed graph defined from a DFA. \n" $<>$

"ToDigraph[Regex[...]] is a directed graph defined from a " <>

"symbolic regular expression. \n" <>

"ToDigraph[RRGrammar[...] is a directed graph defined " <>

"from a right regular grammar.";

RegStar: :usage = "RegStar[NFA[...] performs the star operation on an NFA. $\backslash n$ " $<>$

"RegStar[DFA[...]] performs the star operation on a DFA. \n" $<>$

"RegStar[Regex[...]] performs the star operation on a symbolic " <>

"regular expression. \n" <> 
"RegStar[RRGrammar[...]] performs the star operation on a right " <>

"regular grammar. \n" <>

"RegStar[Digraph[...] performs the star operation on a directed graph.";

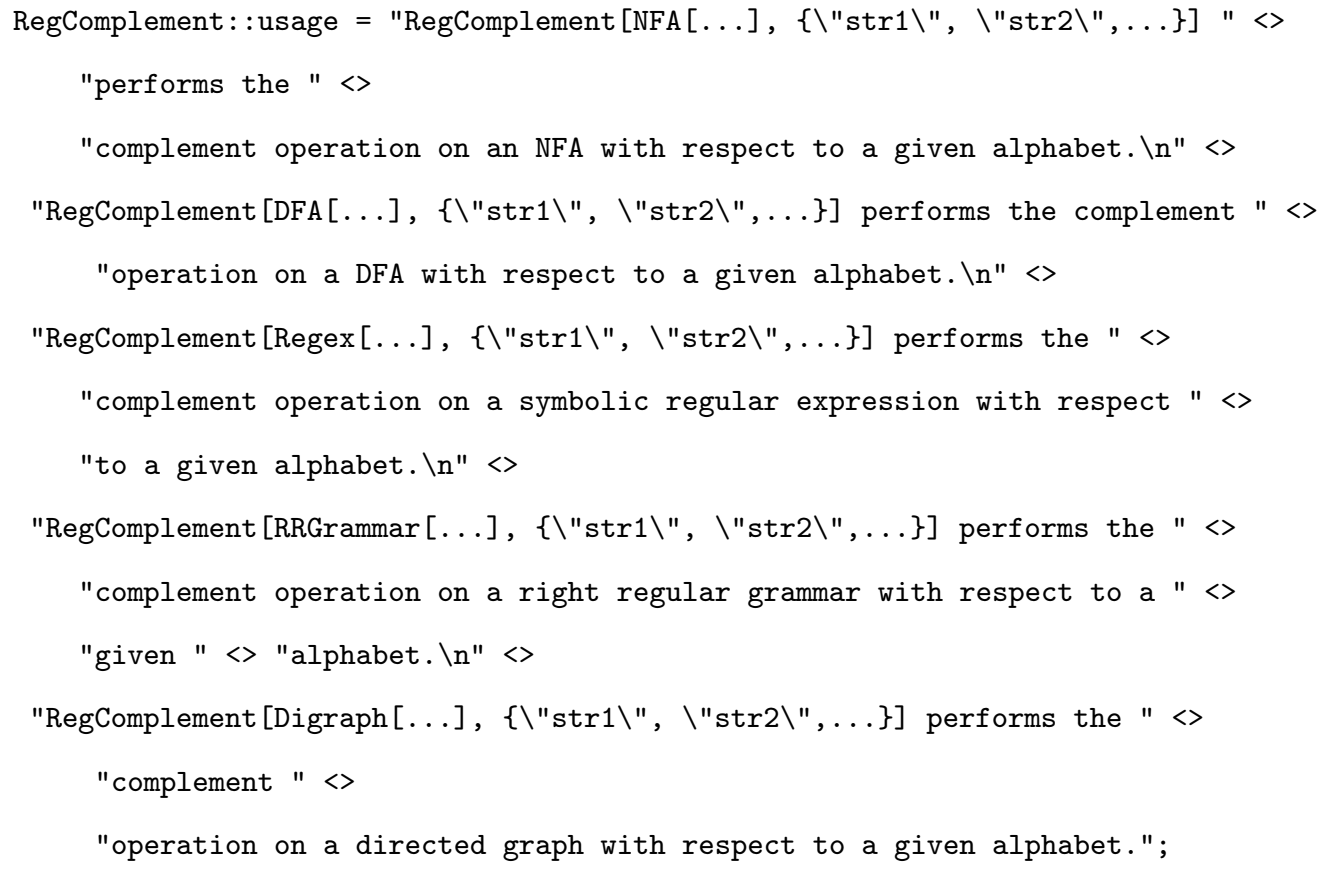


"operation on two NFAs. \n" <>

"RegUnion[DFA[...], DFA[...] performs the reversal operation on two " $<$

"DFAs. \n" $<>$

"RRegUnionRegex[...], Regex[...] performs the reversal operation on two " $<>$

"symbolic " <> "regular expressions. \n" $<>$

"RegUnion[RRGrammar[...], RRGrammar[...] performs the reversal operation " <>

"on two " <> "right regular grammars. \n" $<>$

"RegUnion[Digraph[...], Digraph[...]] performs the reversal operation on " <>

"two directed " <> "graphs.";

RegConcat: : usage $=$ "RegConcat $[\operatorname{NFA}[\ldots], \operatorname{NFA}[\ldots]]$ performs the $"<>$

"concatenation operation on two NFAs. \n" $<>$

"RegConcat[DFA[...], DFA[...]] performs the concatenation operation on " <>

"two DFAs. \n" $<>$

"ReRegConcategex[...], Regex[...] performs the concatenation operation on " <>

"two symbolic " <> "regular expressions. \n" <>

"RegConcat [RRGrammar [...], RRGrammar[...] performs the concatenation " $<>$

"operation on two " <> "right regular grammars. \n" $<>$

"RegConcat[Digraph[...], Digraph[...]] performs the concatenation " $<$

"operation on two directed " <> "graphs.";

RegIntersection: : usage $=$ "RegIntersection[NFA[...], NFA[...] performs the " $<$

"intersection operation on two NFAs. \n" $<>$

"RegIntersection[DFA[...], DFA[...]] performs the intersection operation " <>

"on two DFAs. \n" $<>$

"RegIntersection[Regex[...], Regex[...]] performs the intersection " $<>$

"operation on two symbolic " <>

"regular expressions. \n" $<$

"RegIntersection[RRGrammar[...], RRGrammar[...] performs the intersection " <> "operation on two " <> "right regular grammars. In" $<>$ 


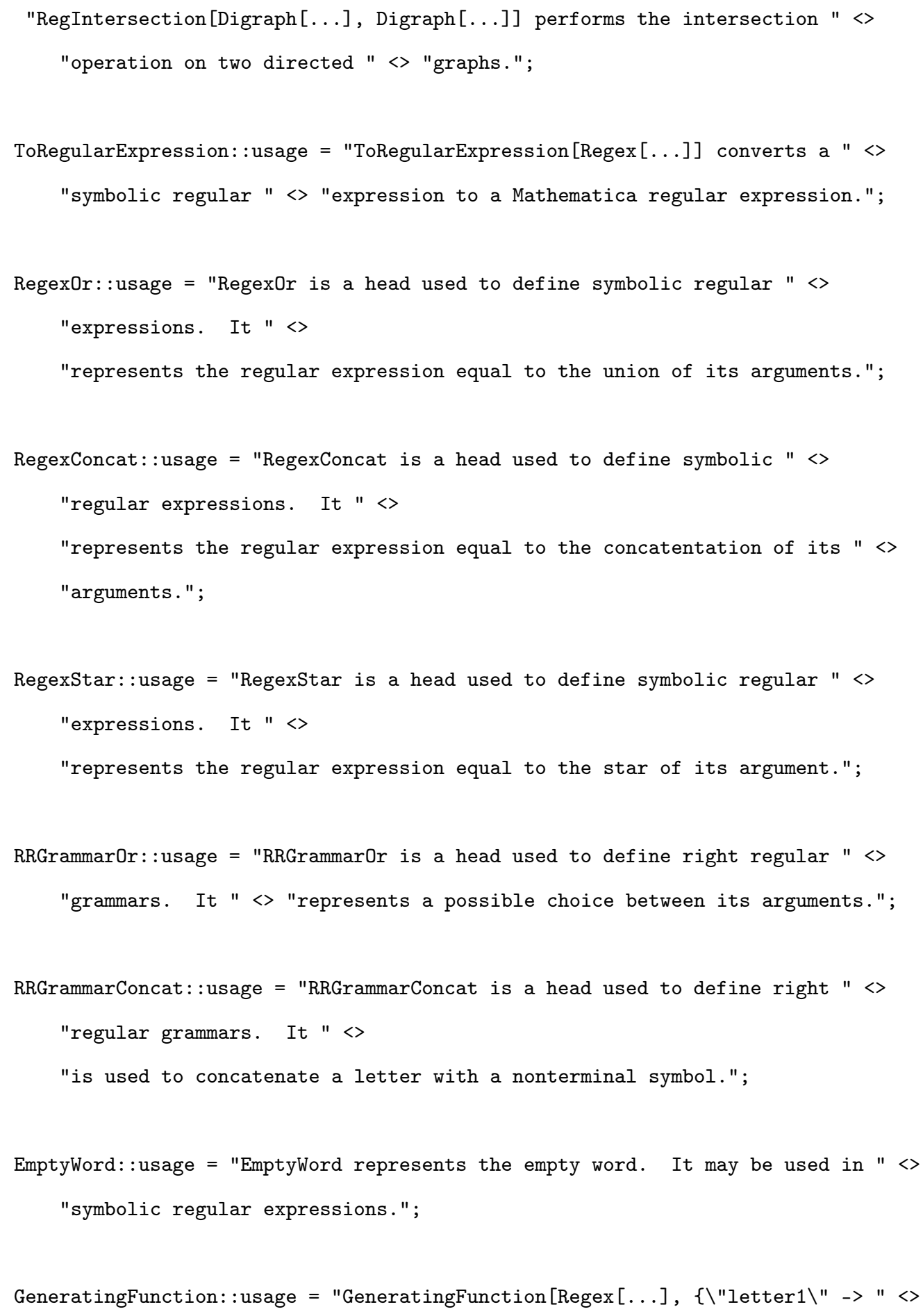




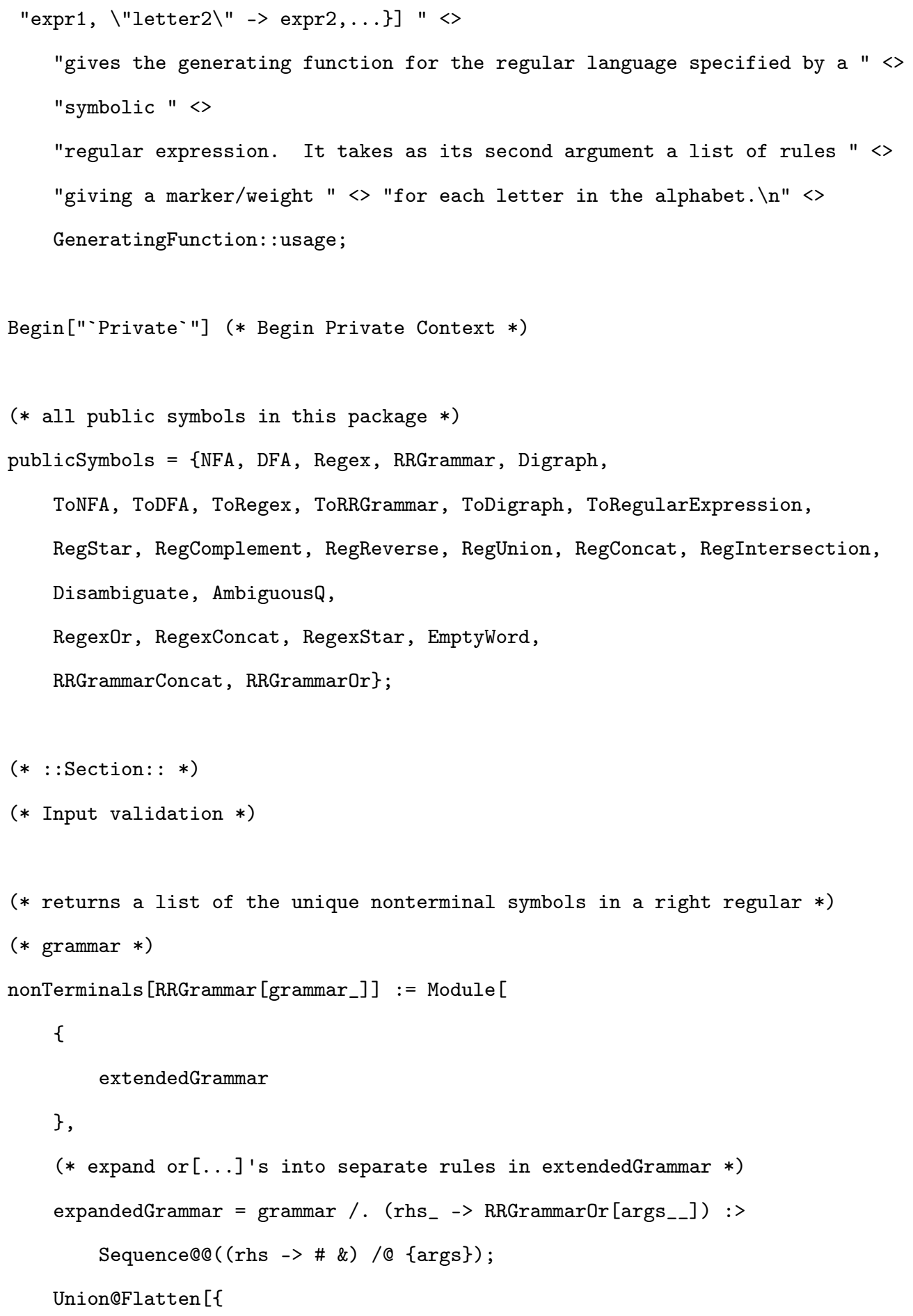









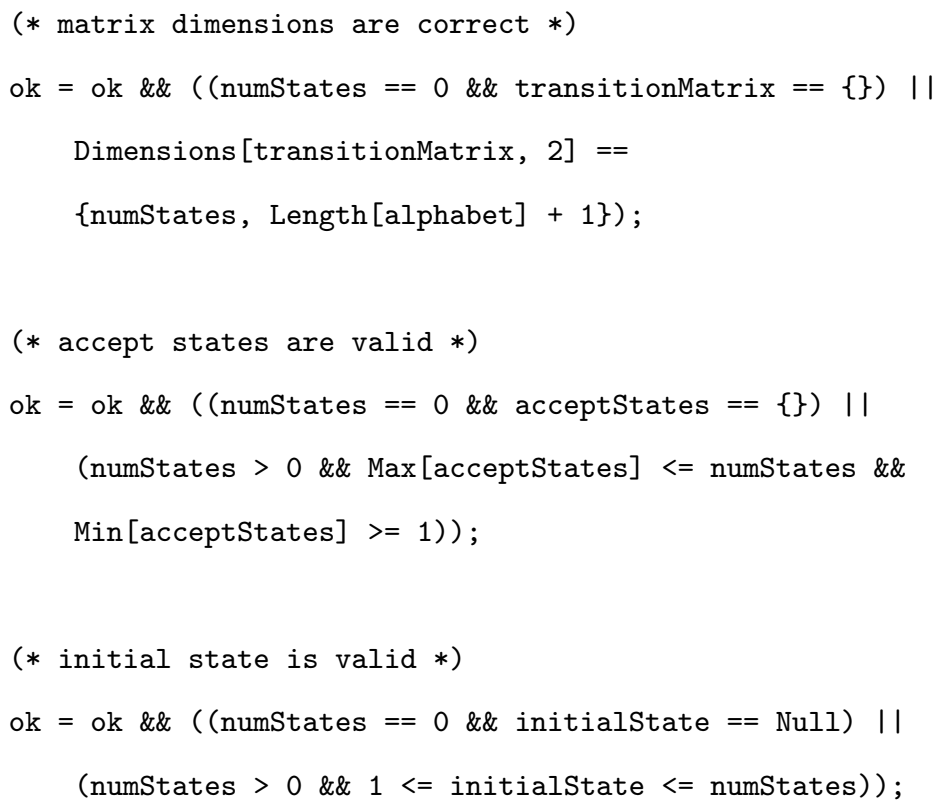




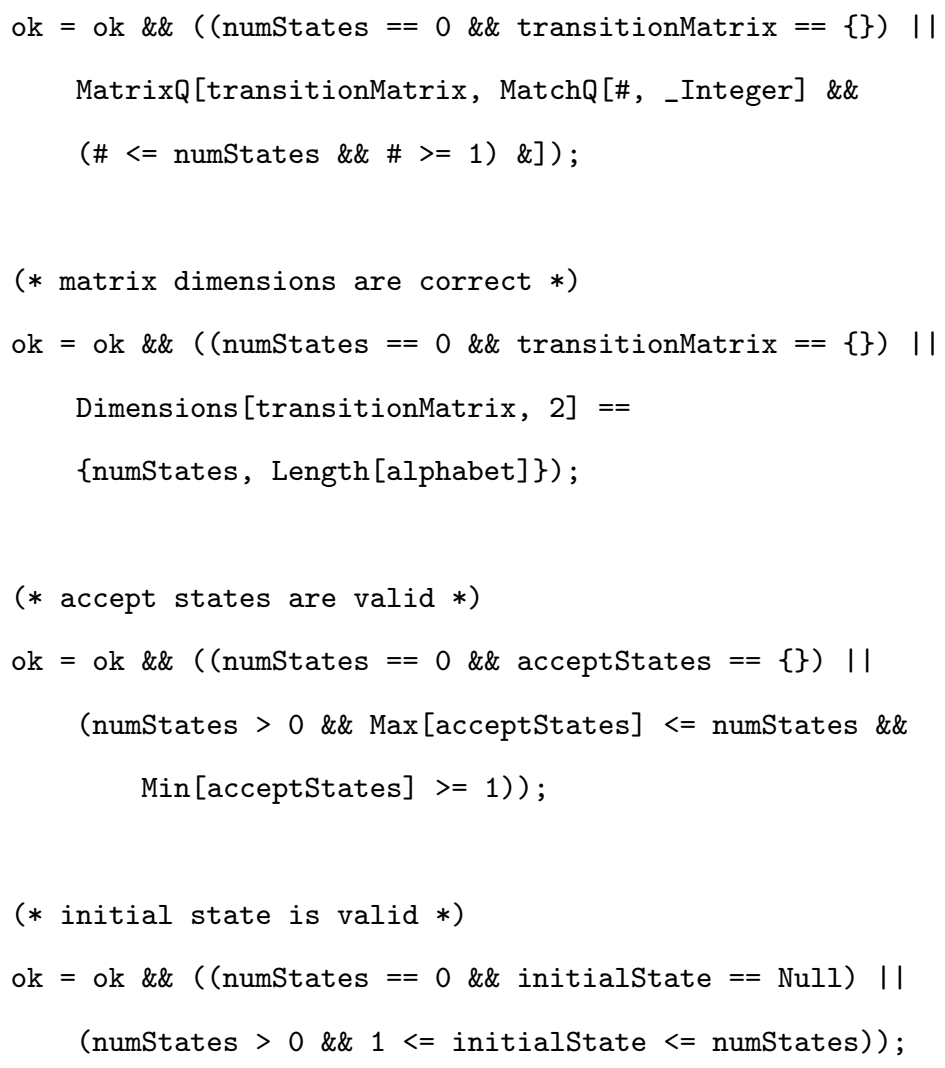




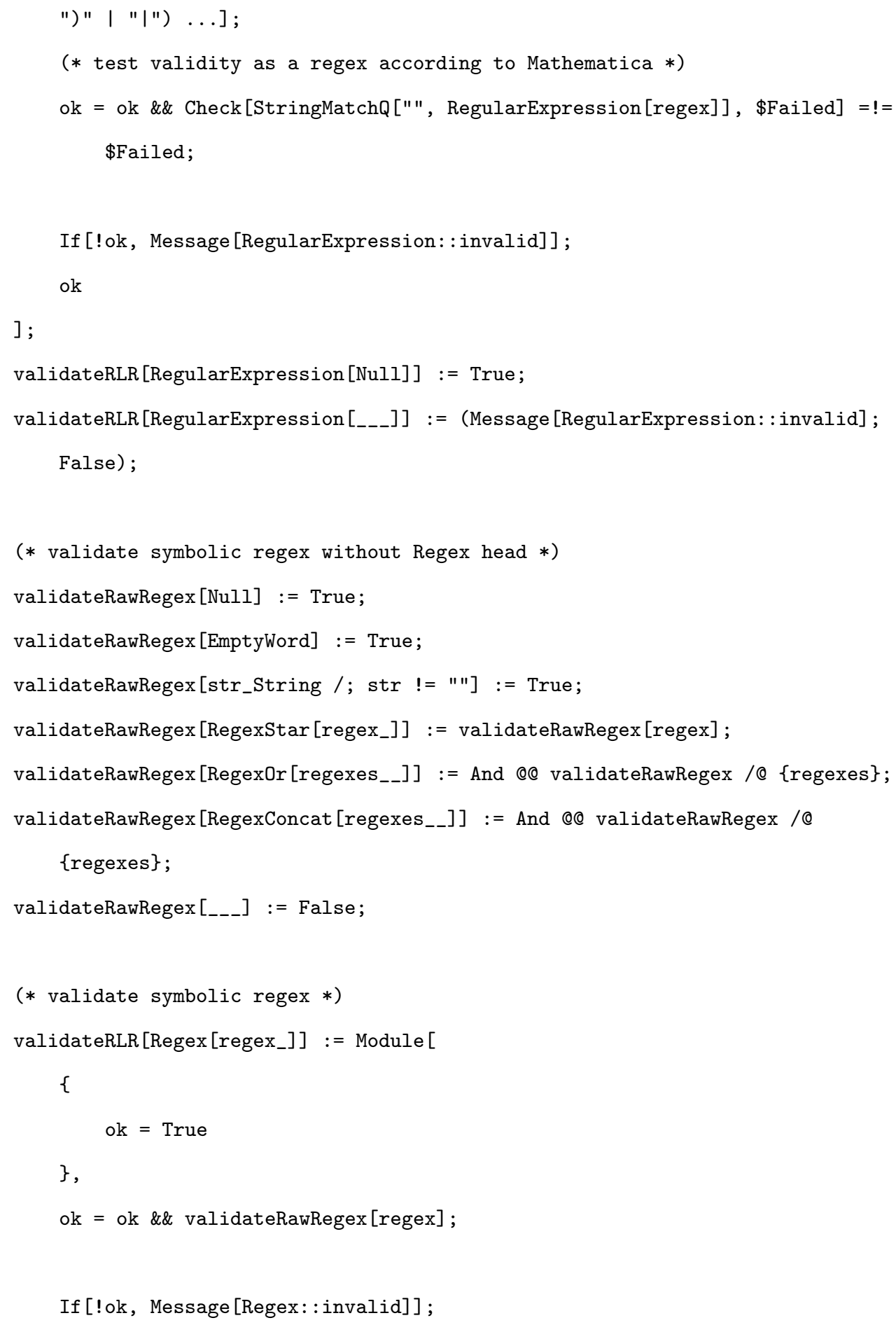




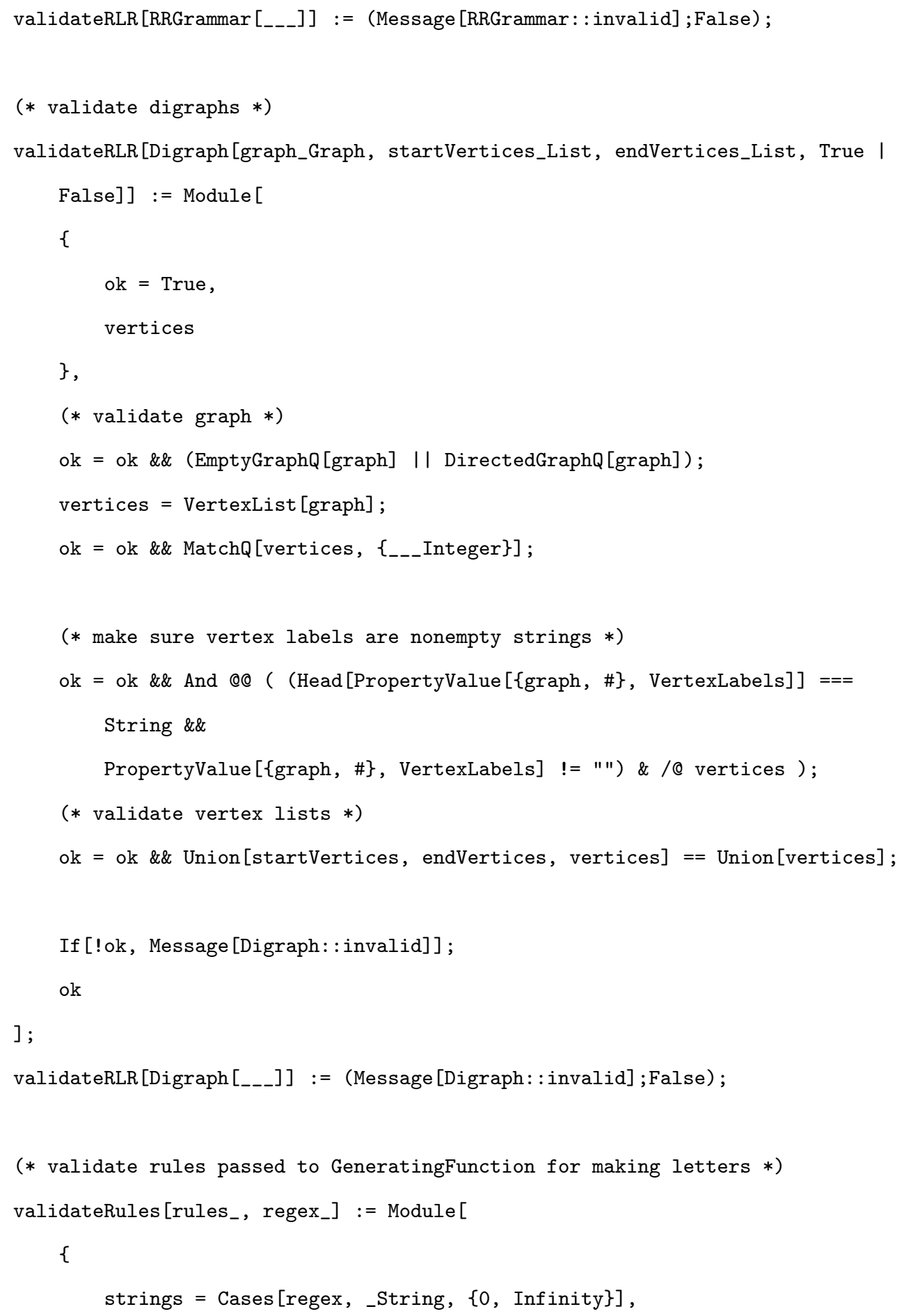




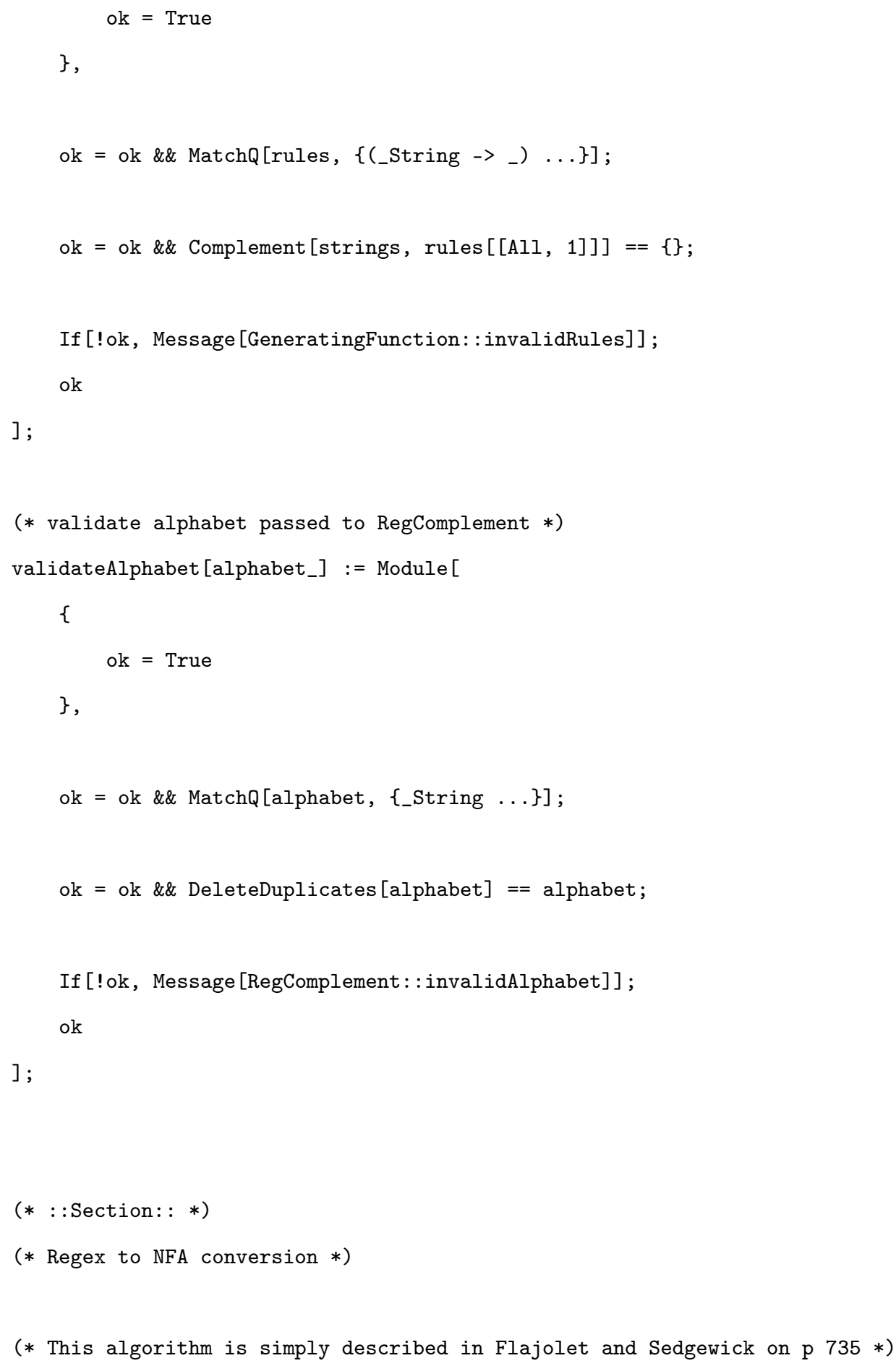




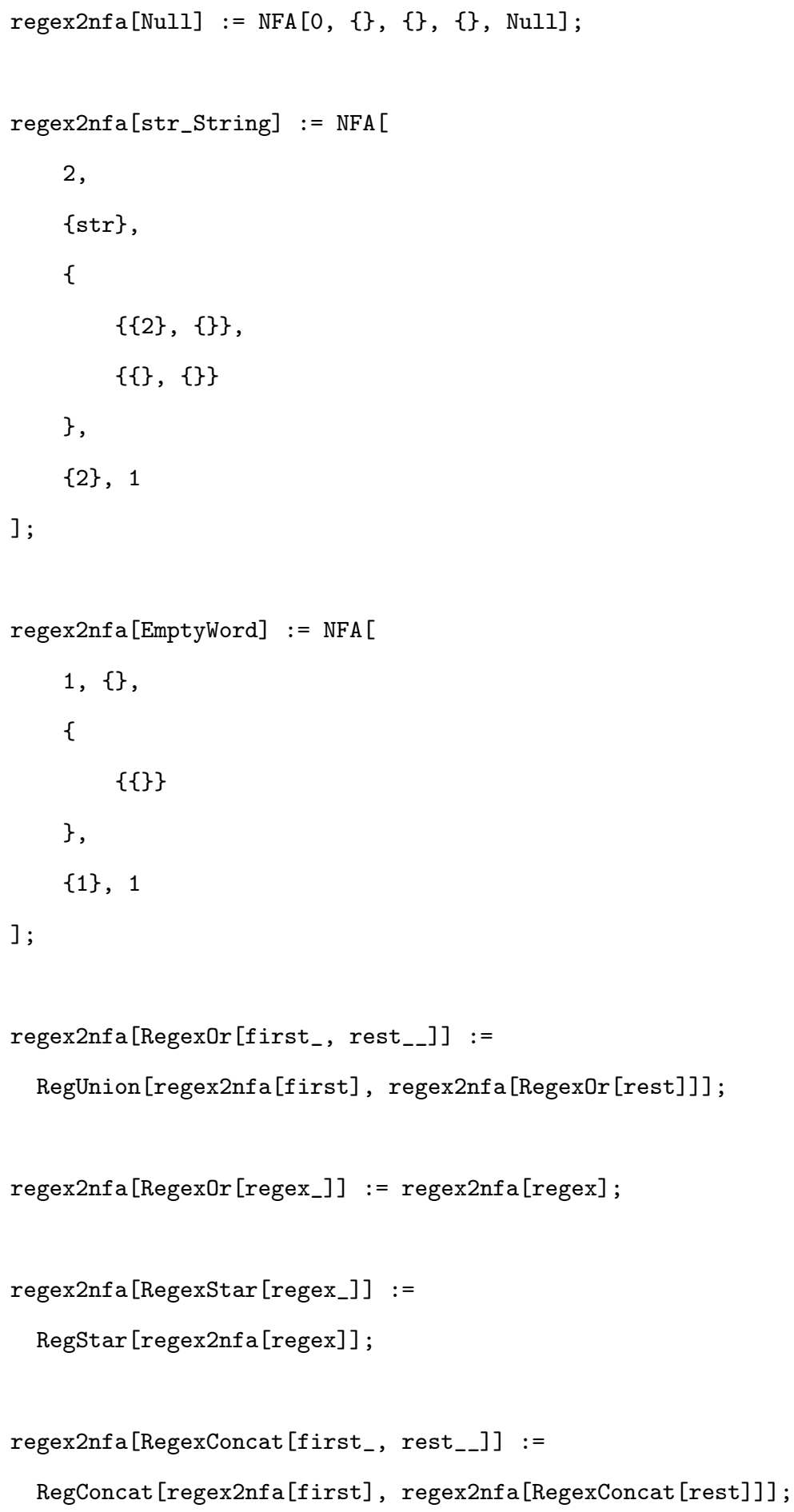




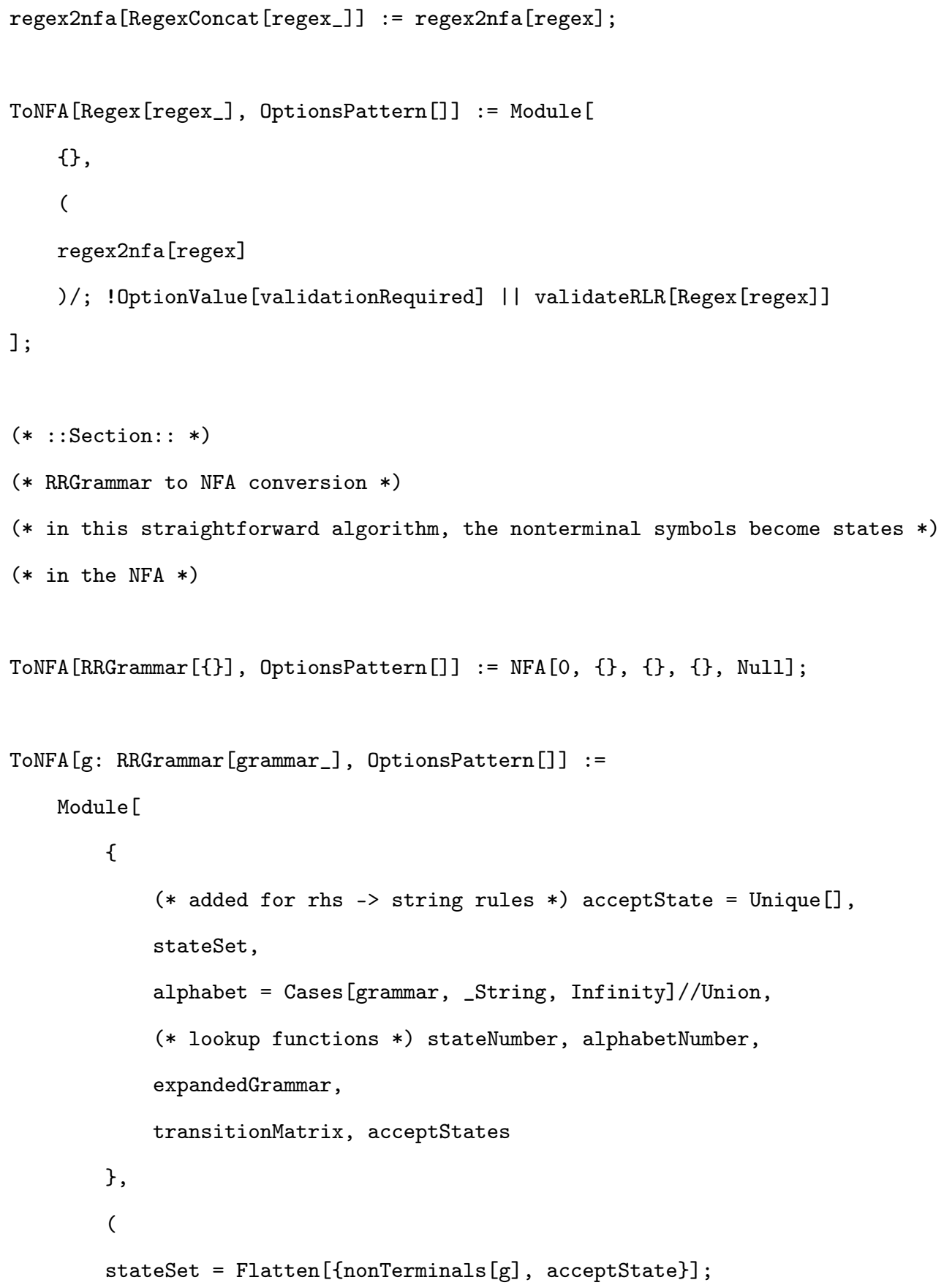




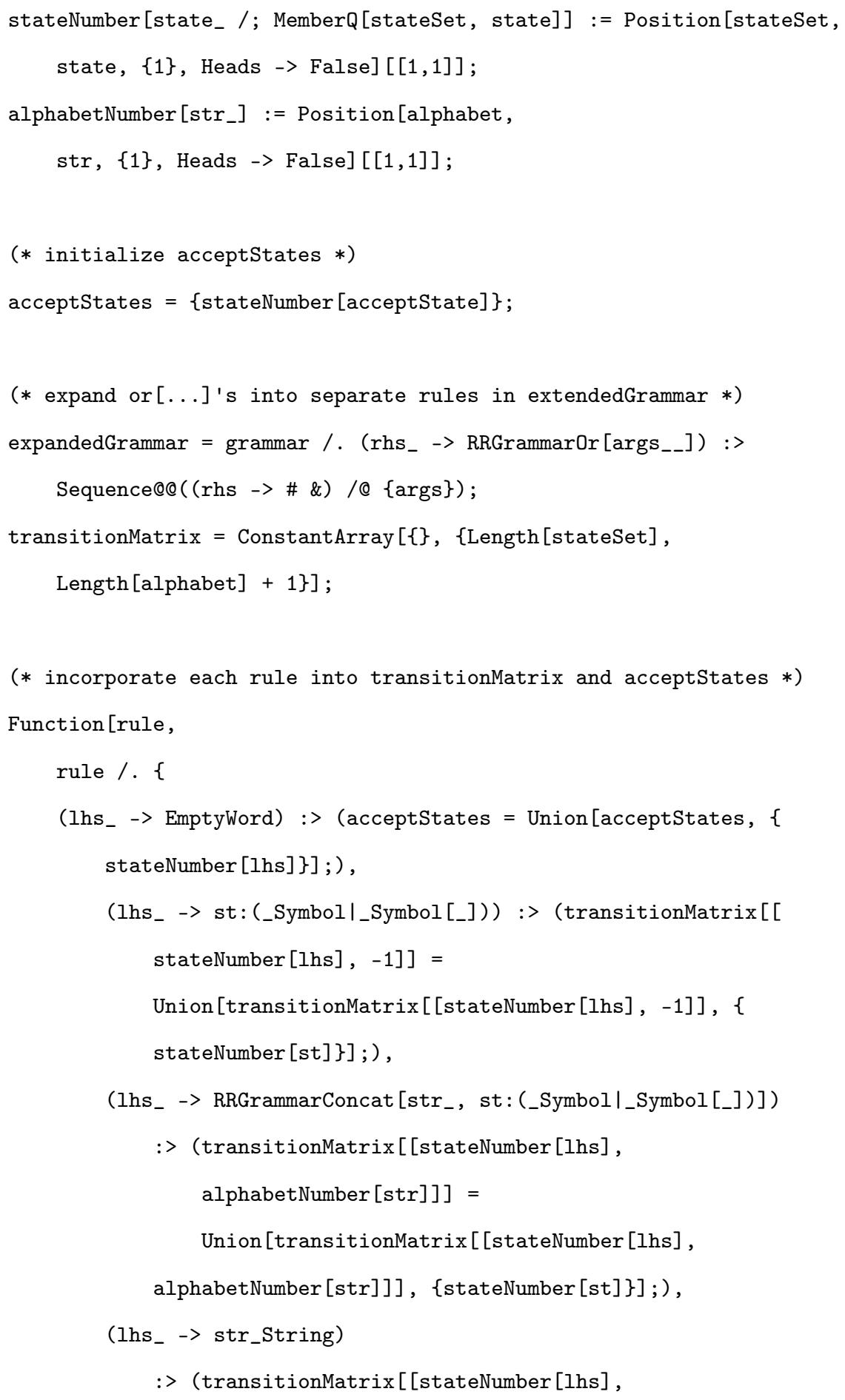




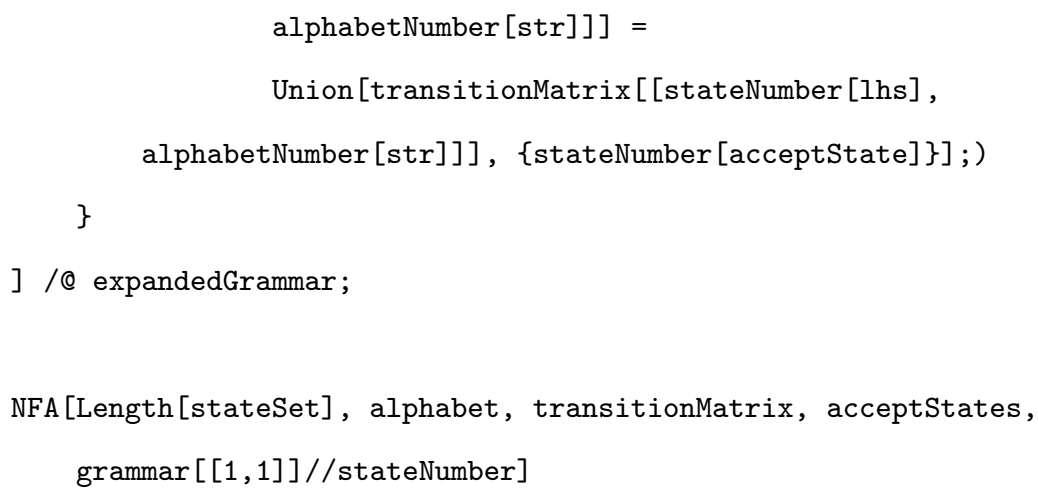




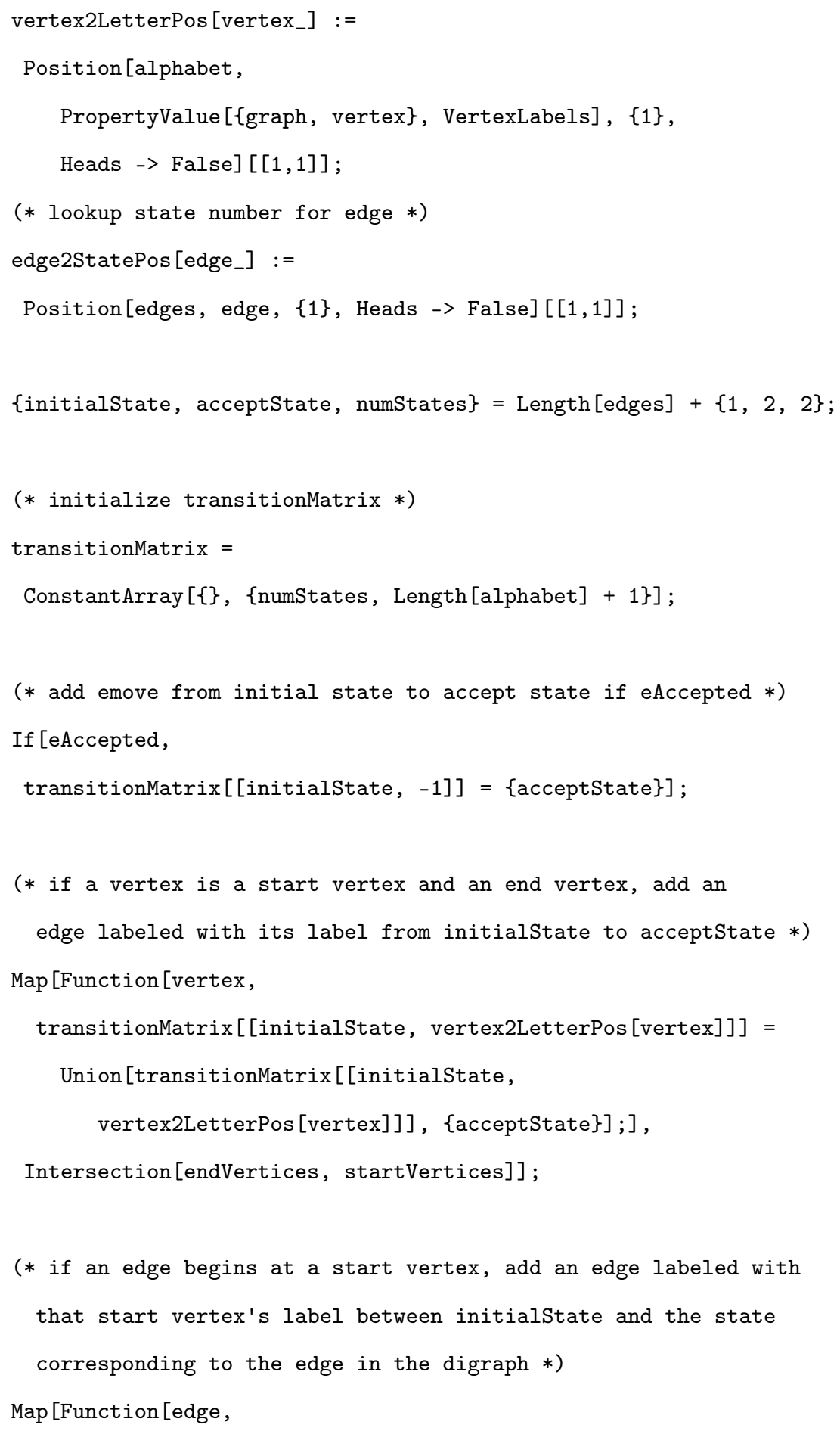




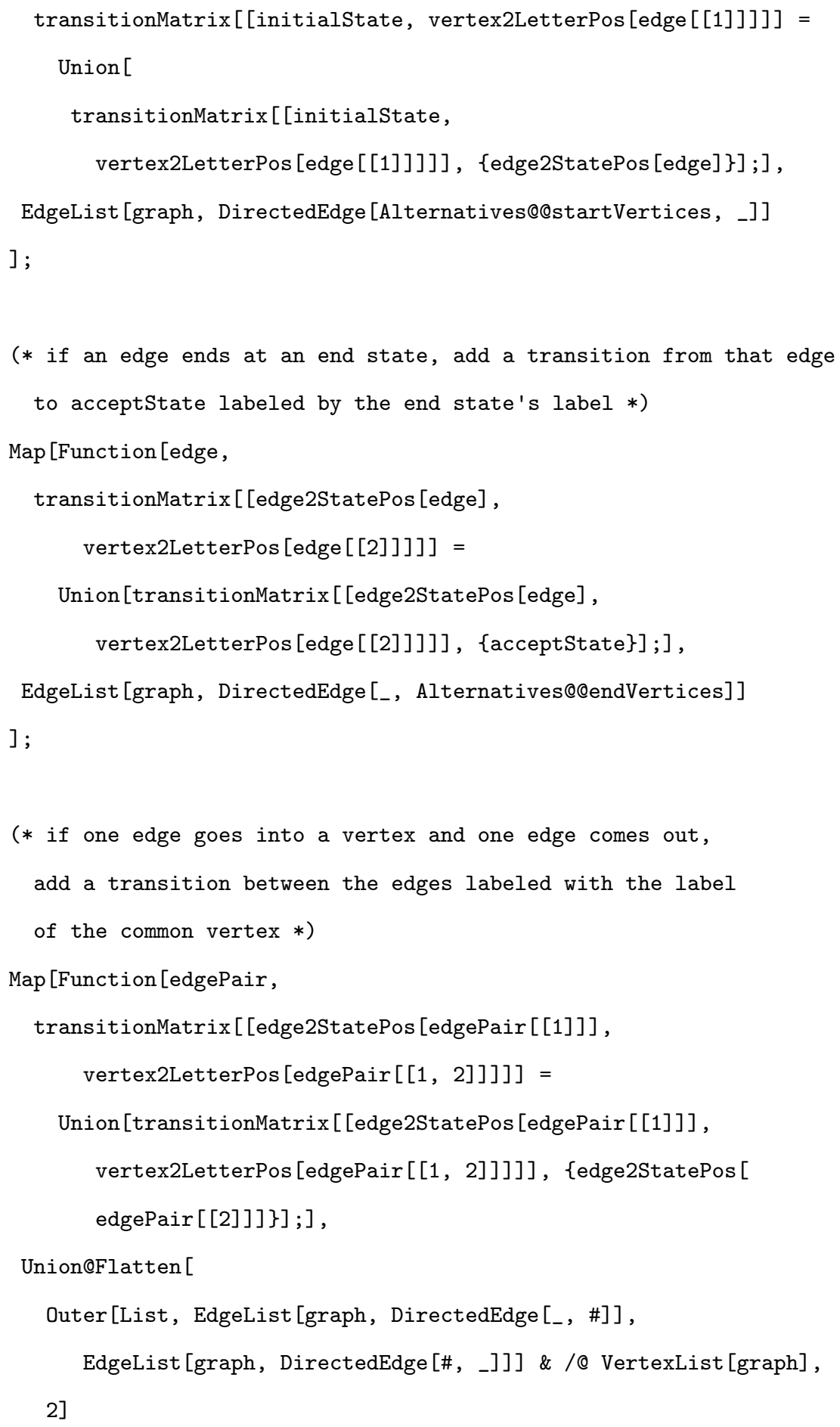




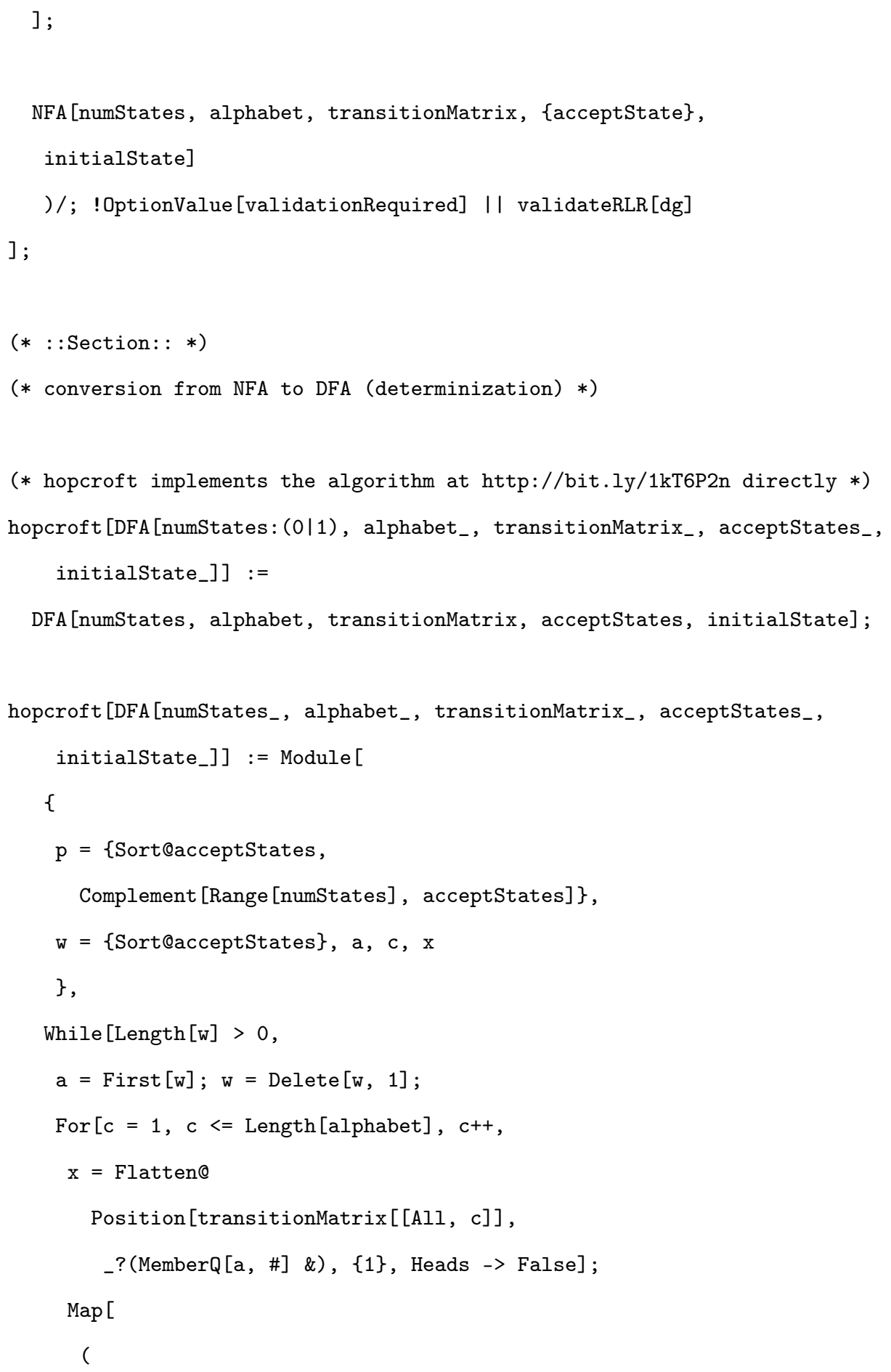




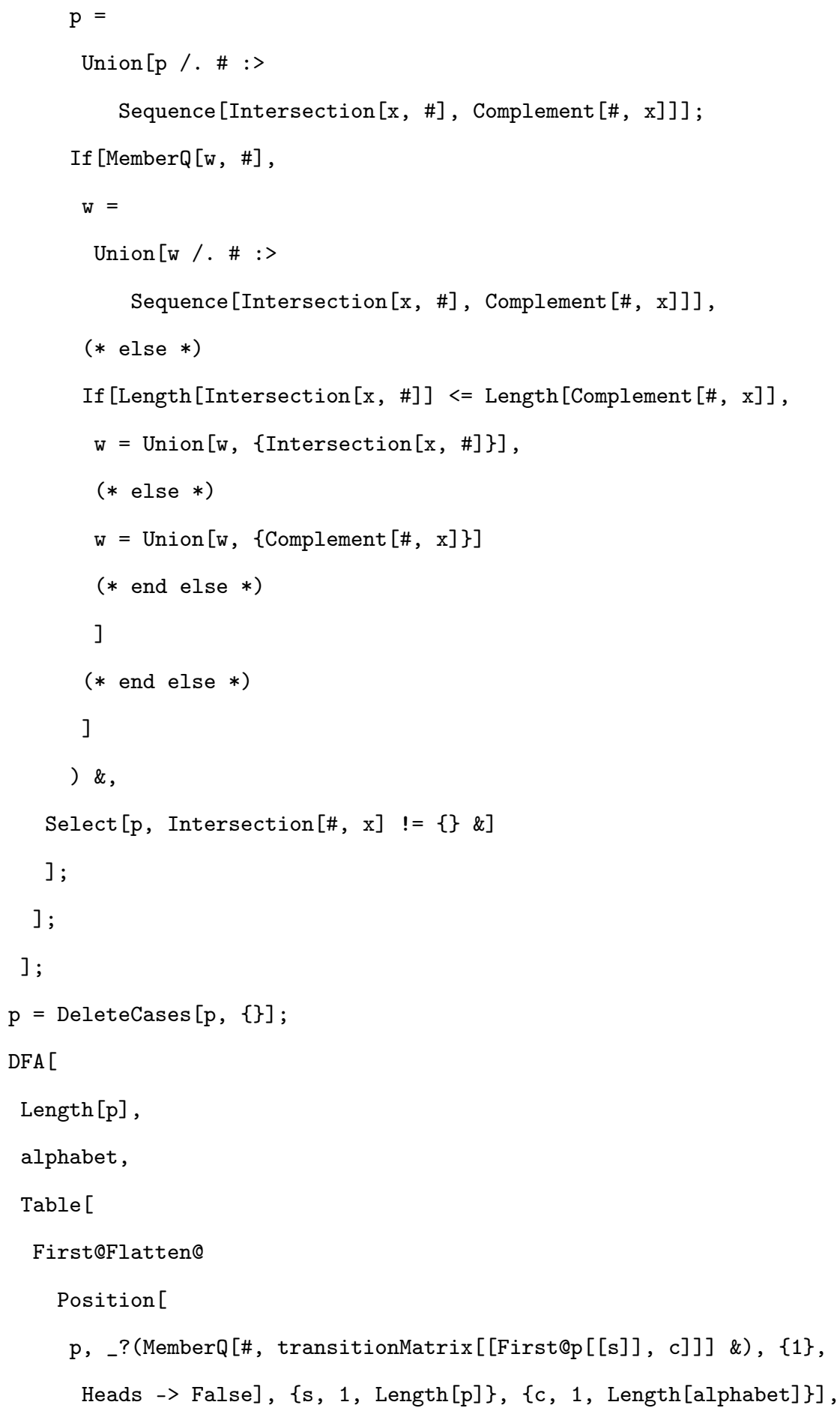




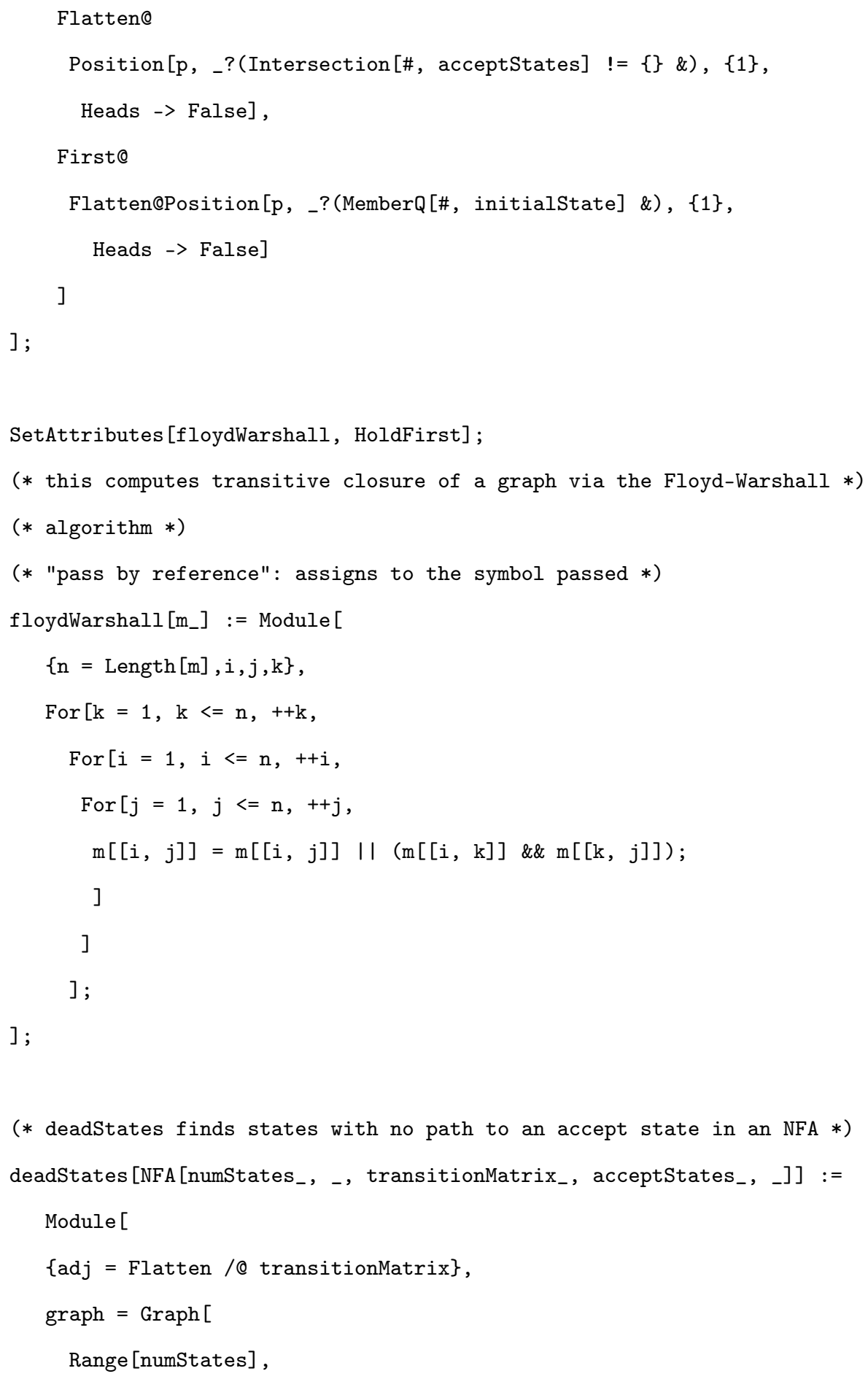




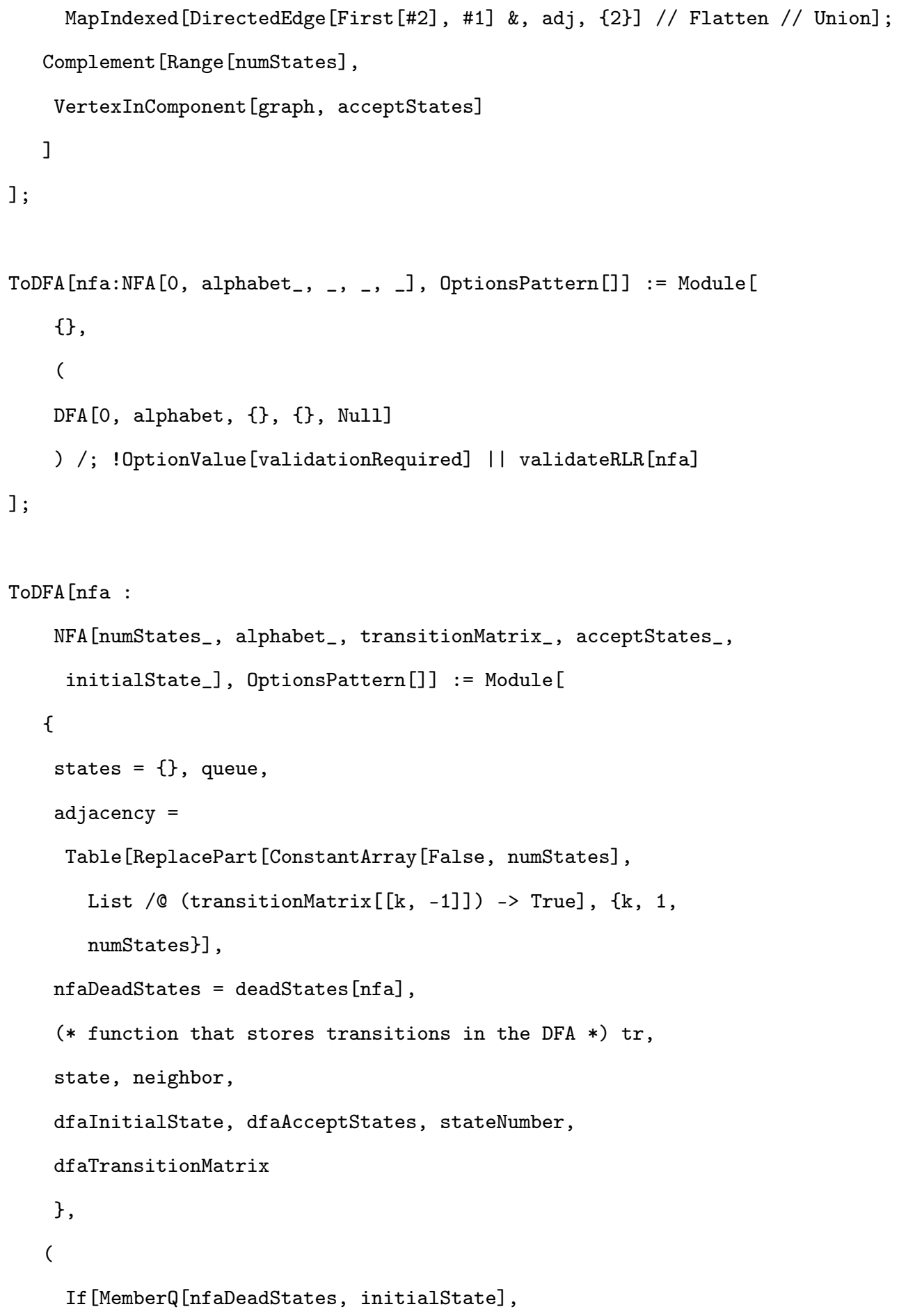




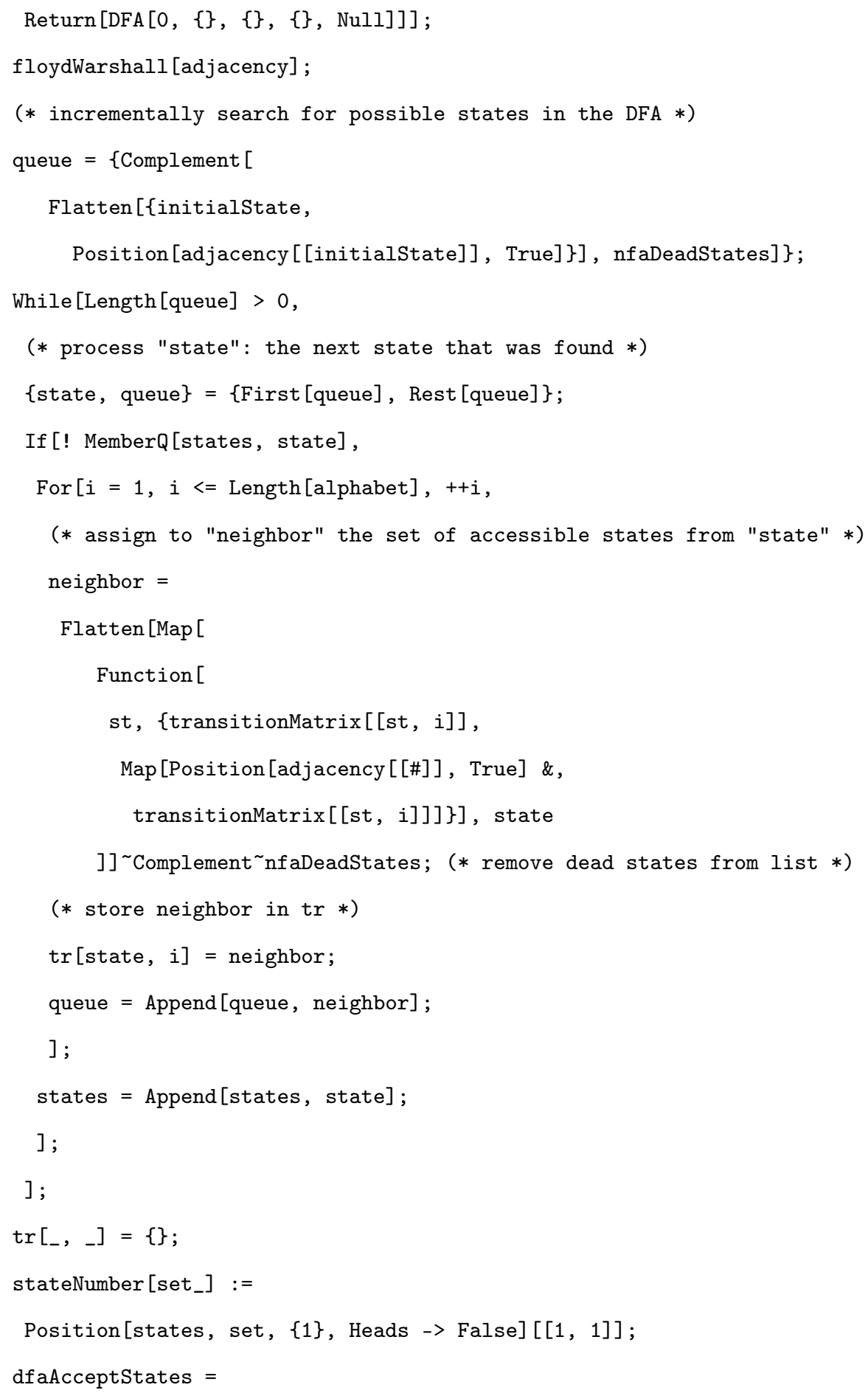




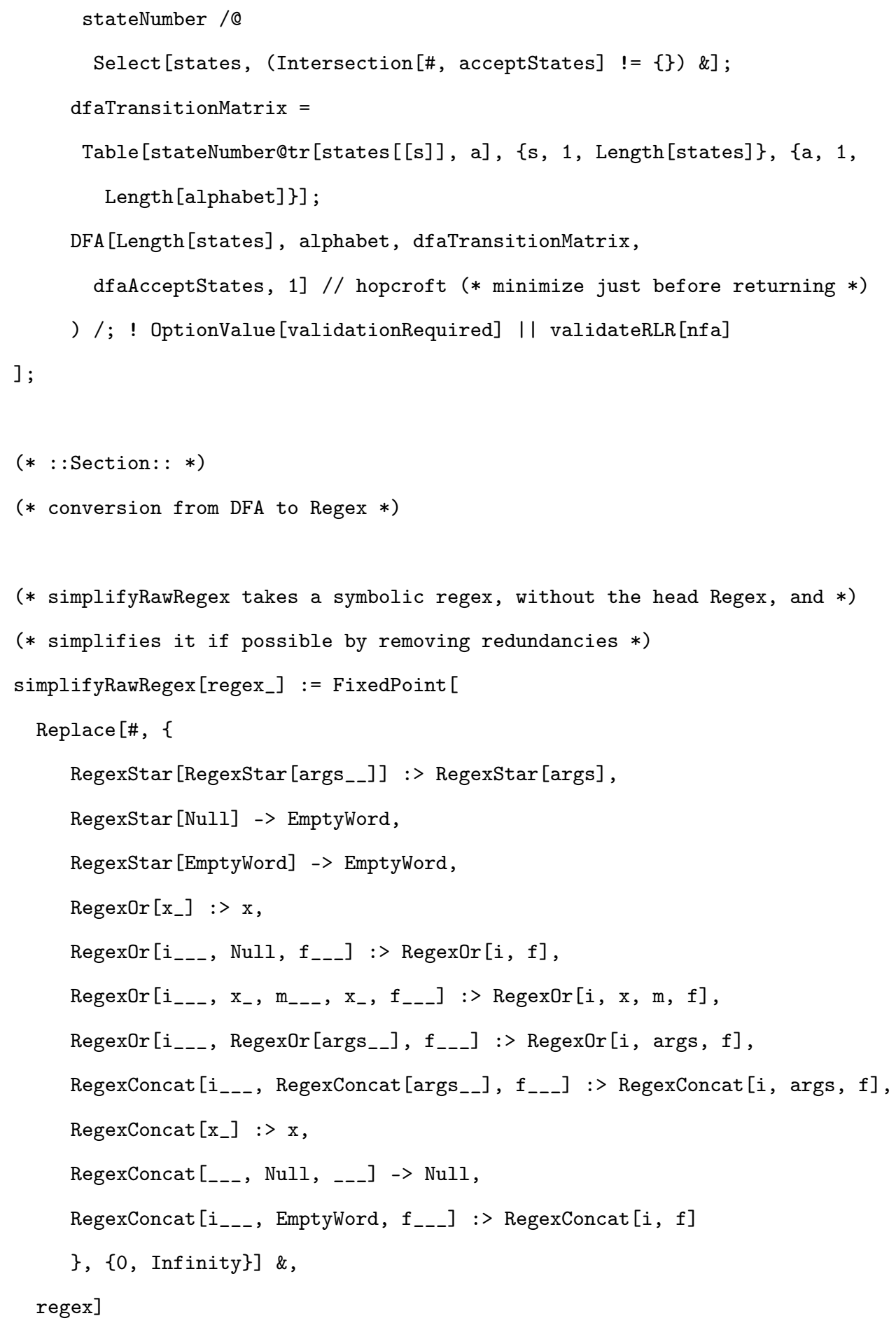




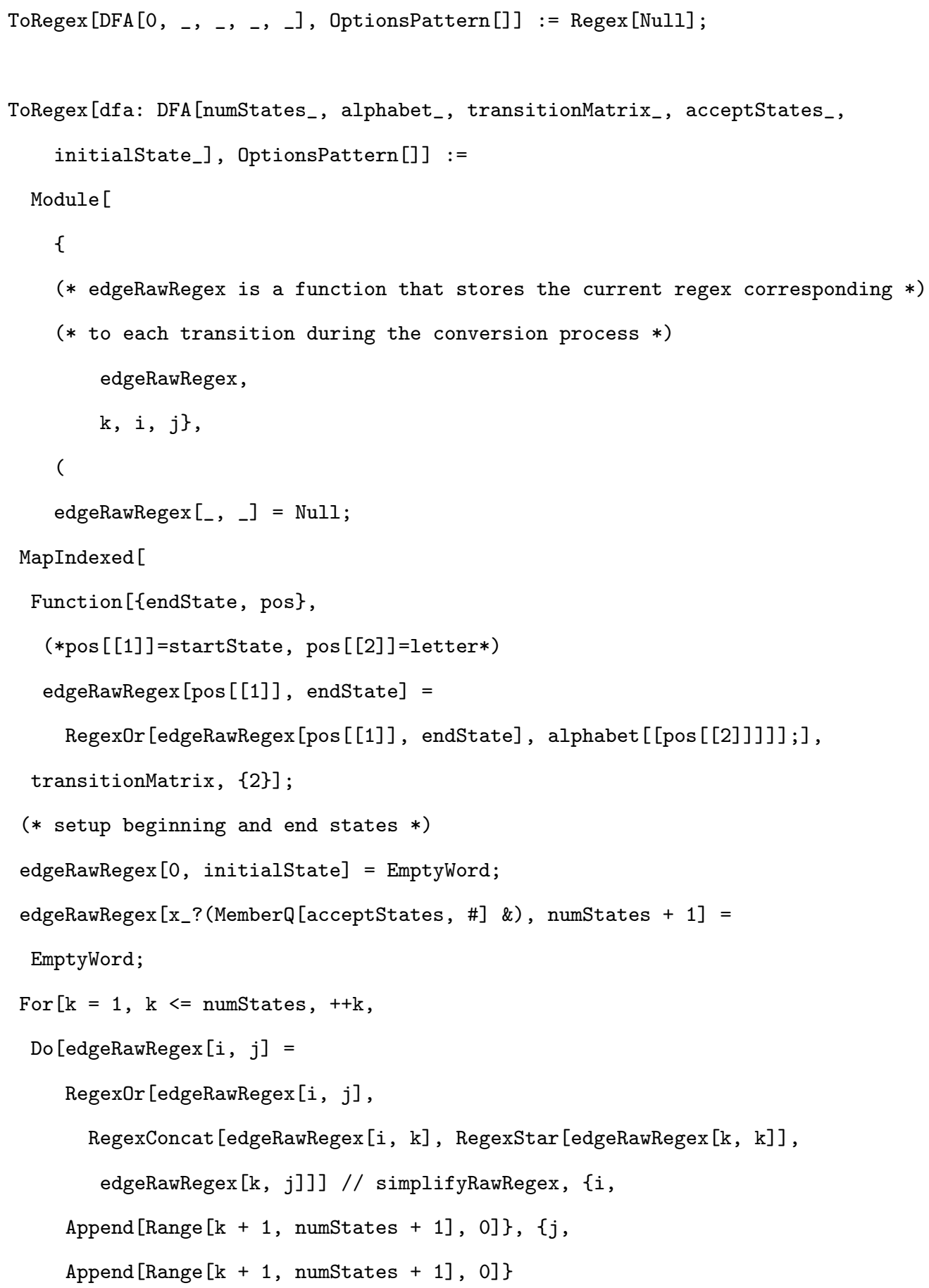




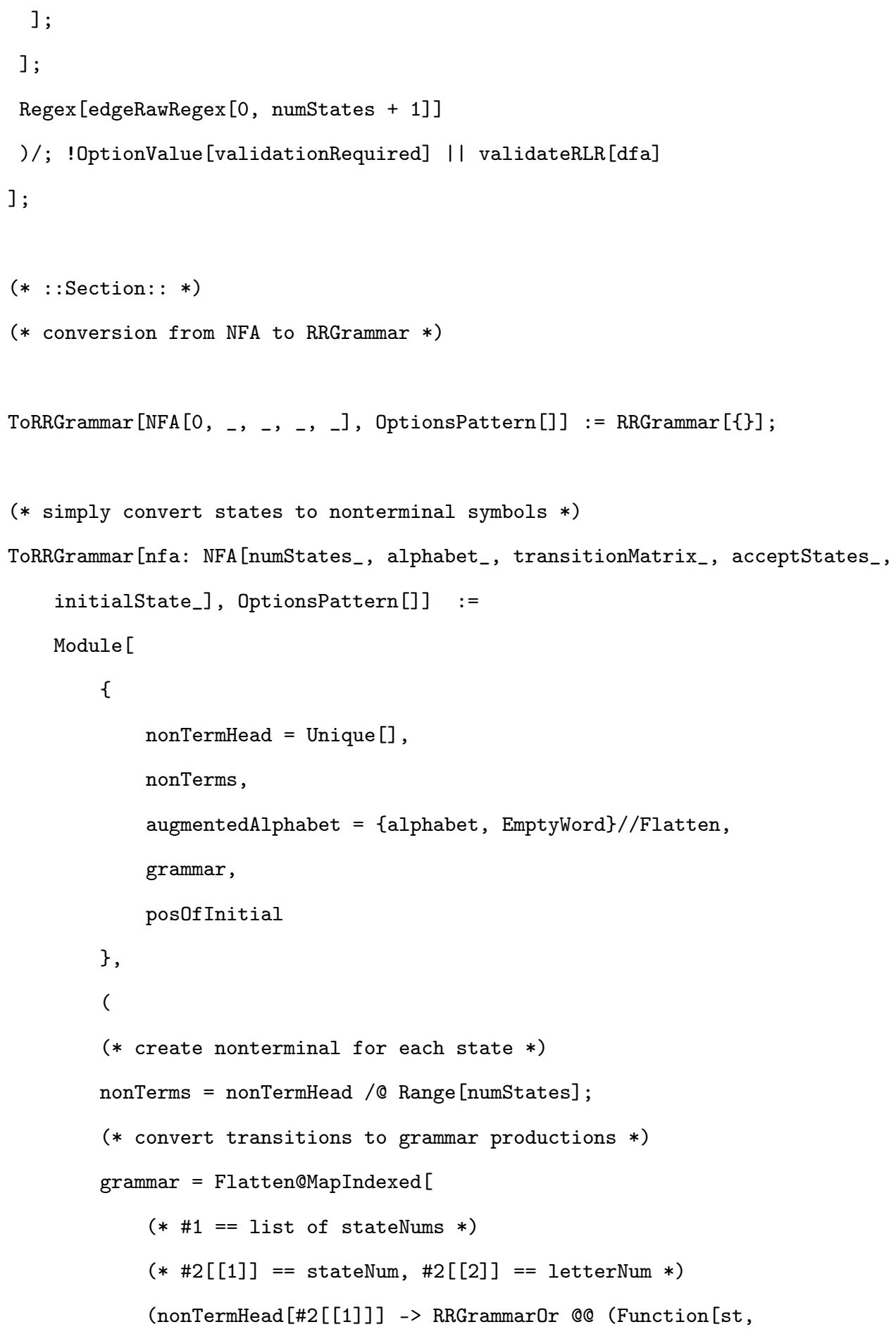




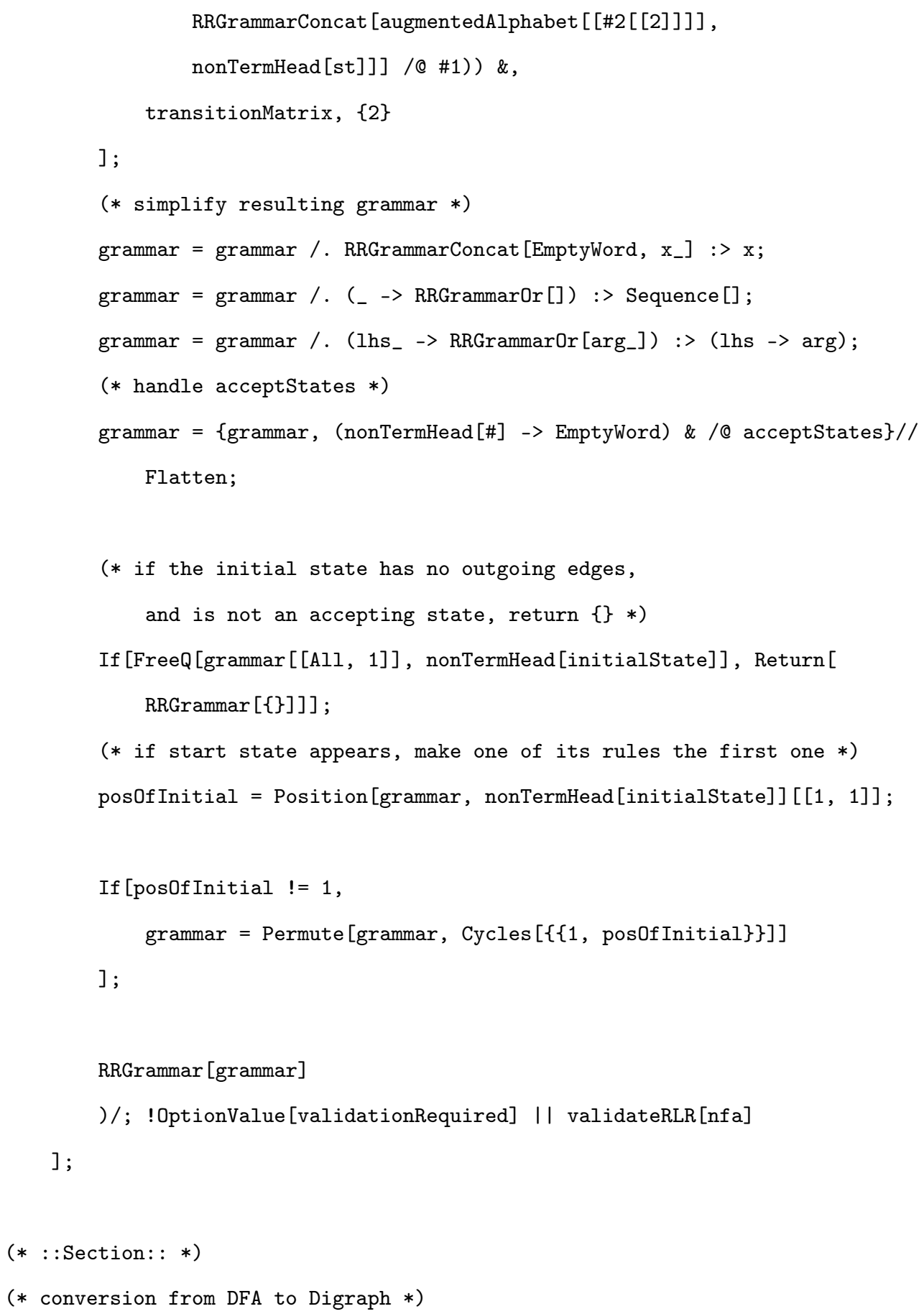




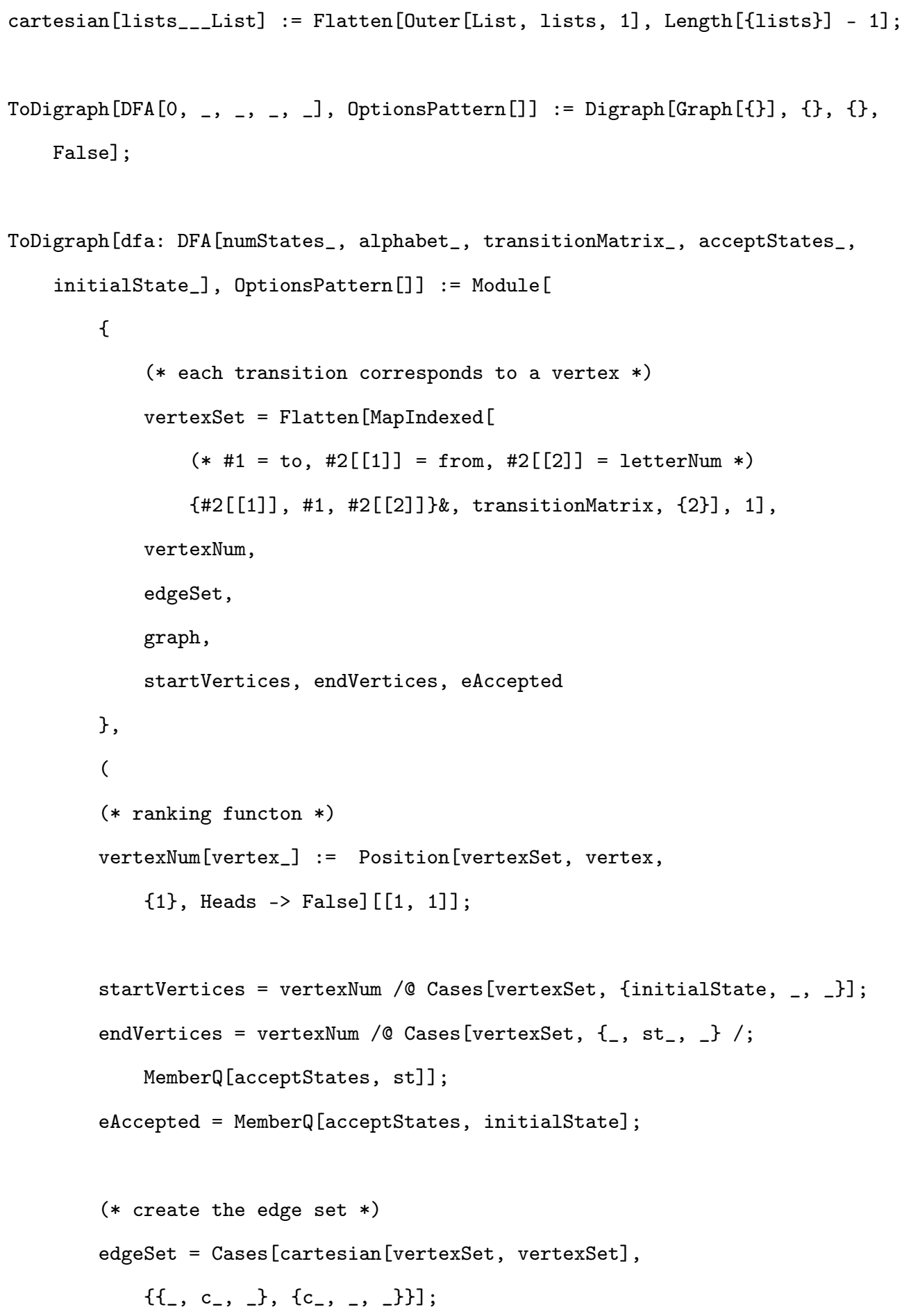




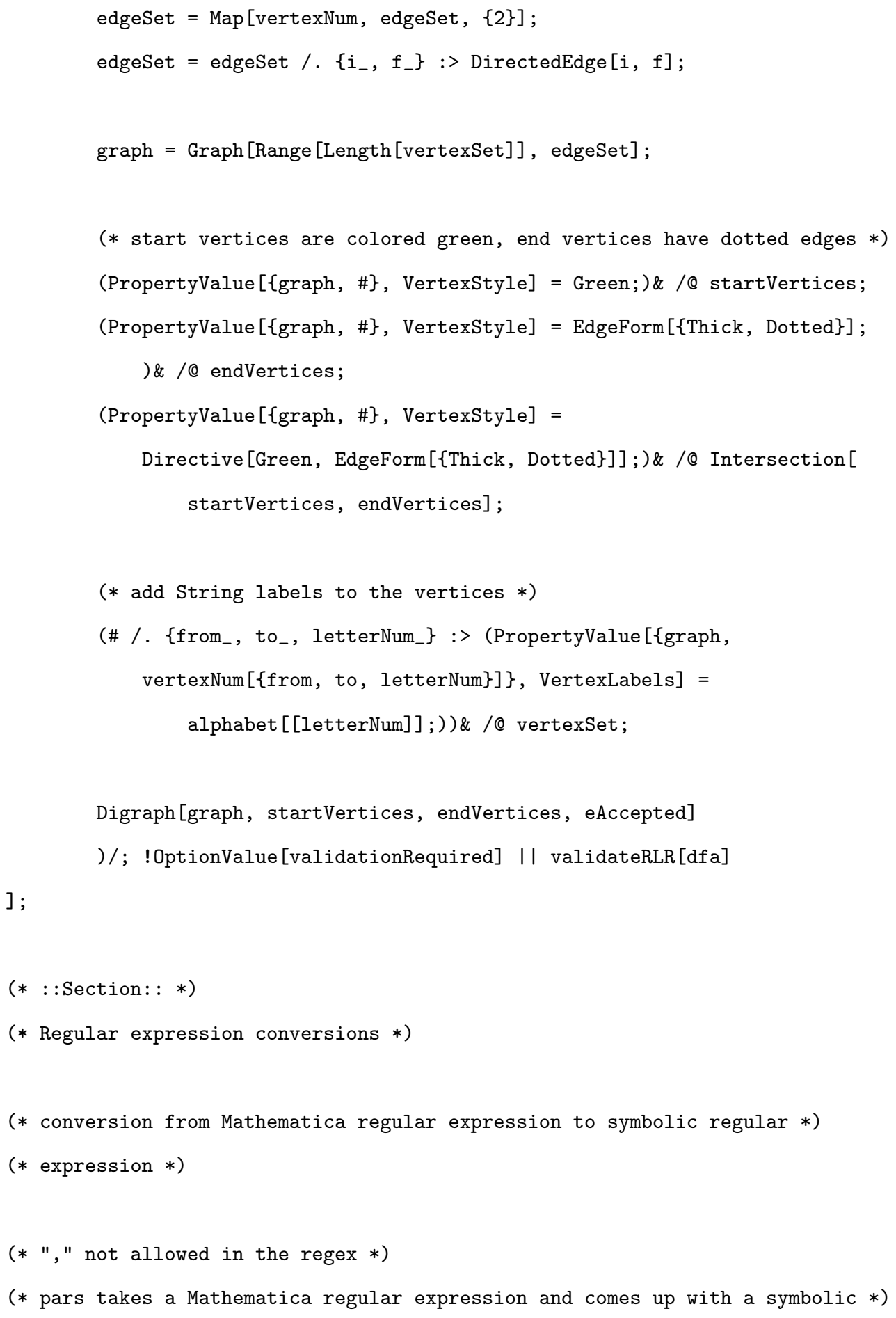




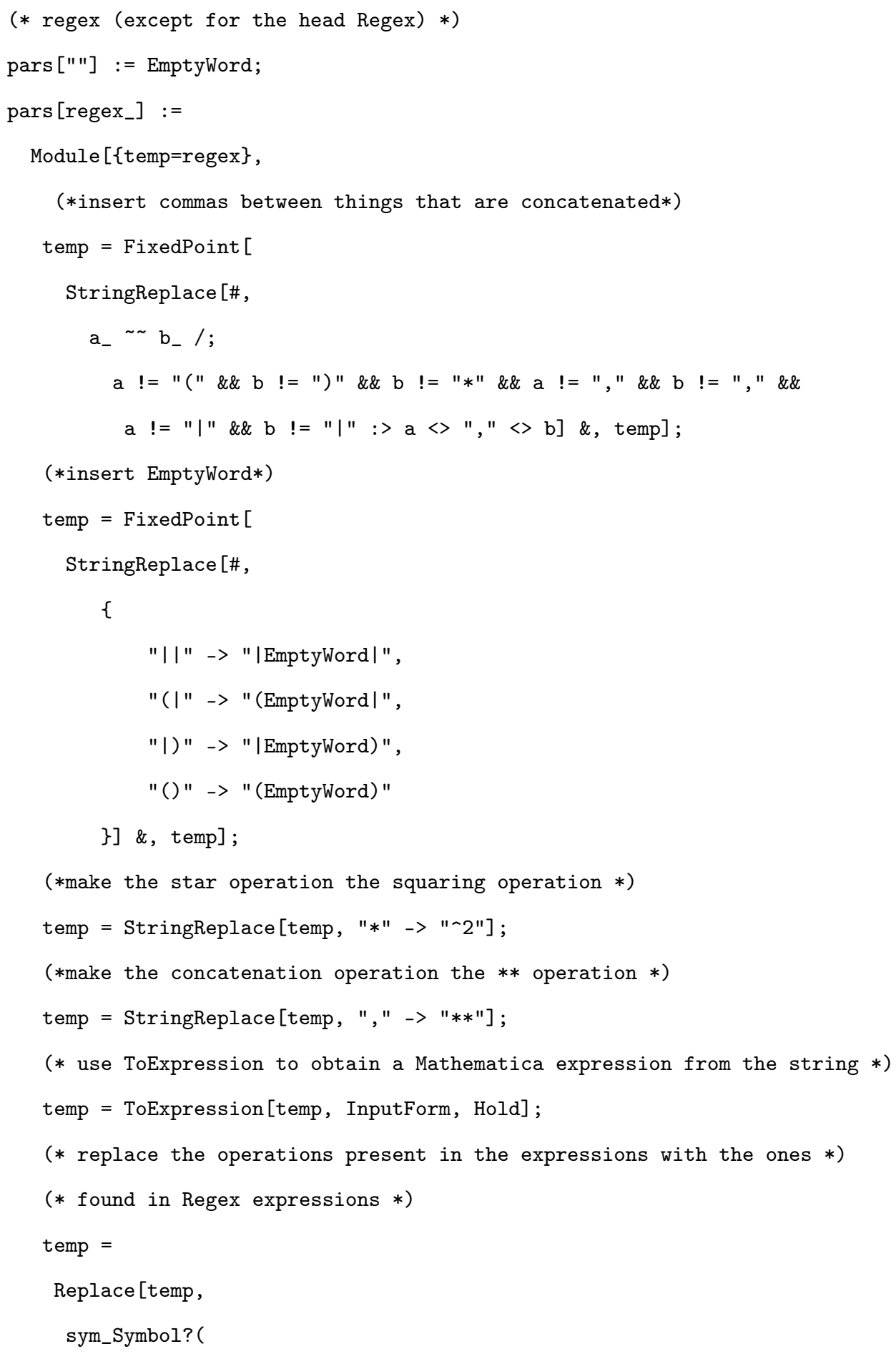

\}] \&, temp] ;

(*make the star operation the squaring operation $*$ )

temp = StringReplace[temp, "*" $->$ " 2"];

( $*$ make the concatenation operation the $* *$ operation $*$ )

temp = StringReplace[temp, "," -> "**"];

(* use ToExpression to obtain a Mathematica expression from the string *)

temp $=$ ToExpression [temp, InputForm, Hold];

(* replace the operations present in the expressions with the ones *)

(* found in Regex expressions *)

temp $=$

Replace [temp,

sym_Symbol? ( 


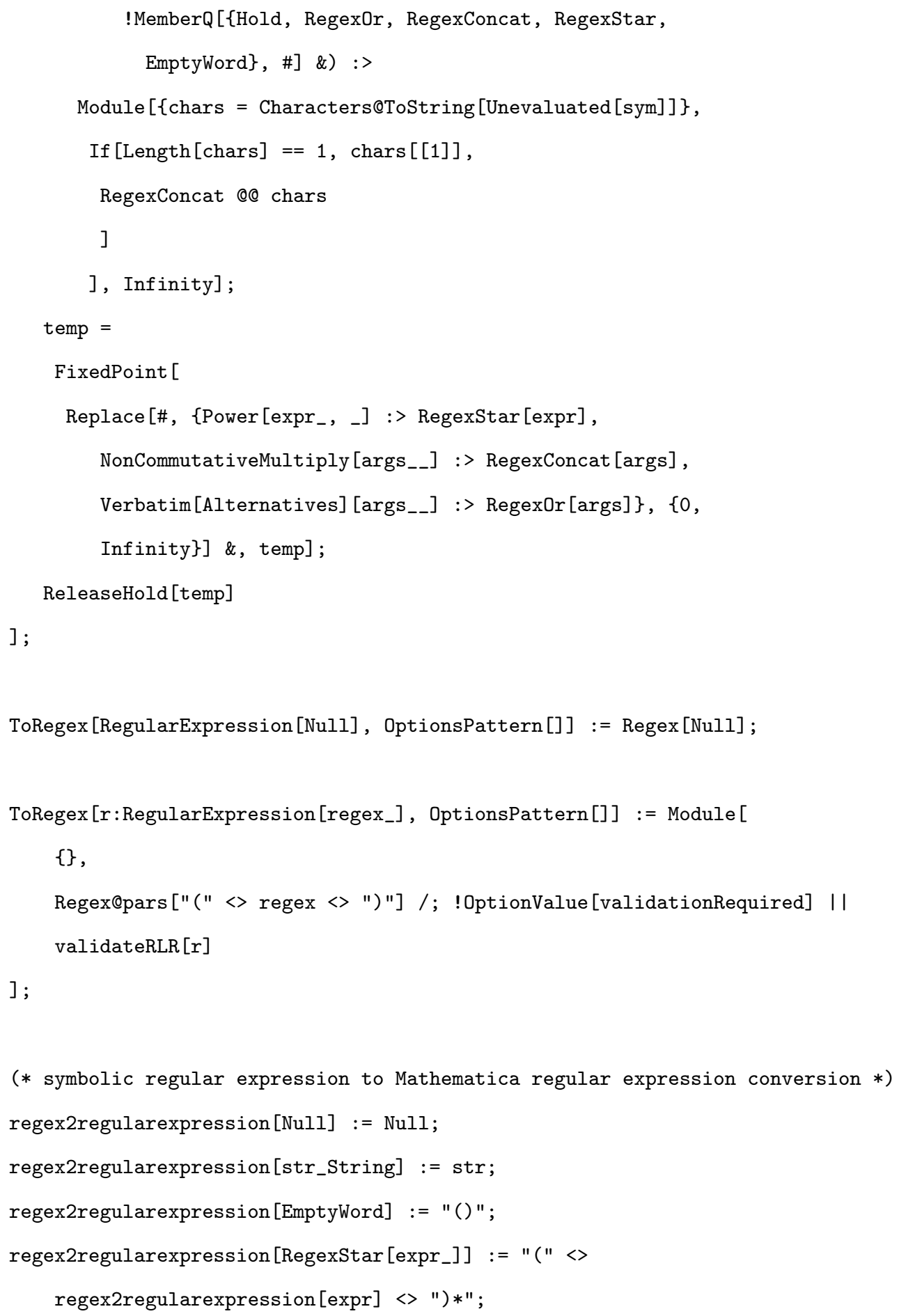




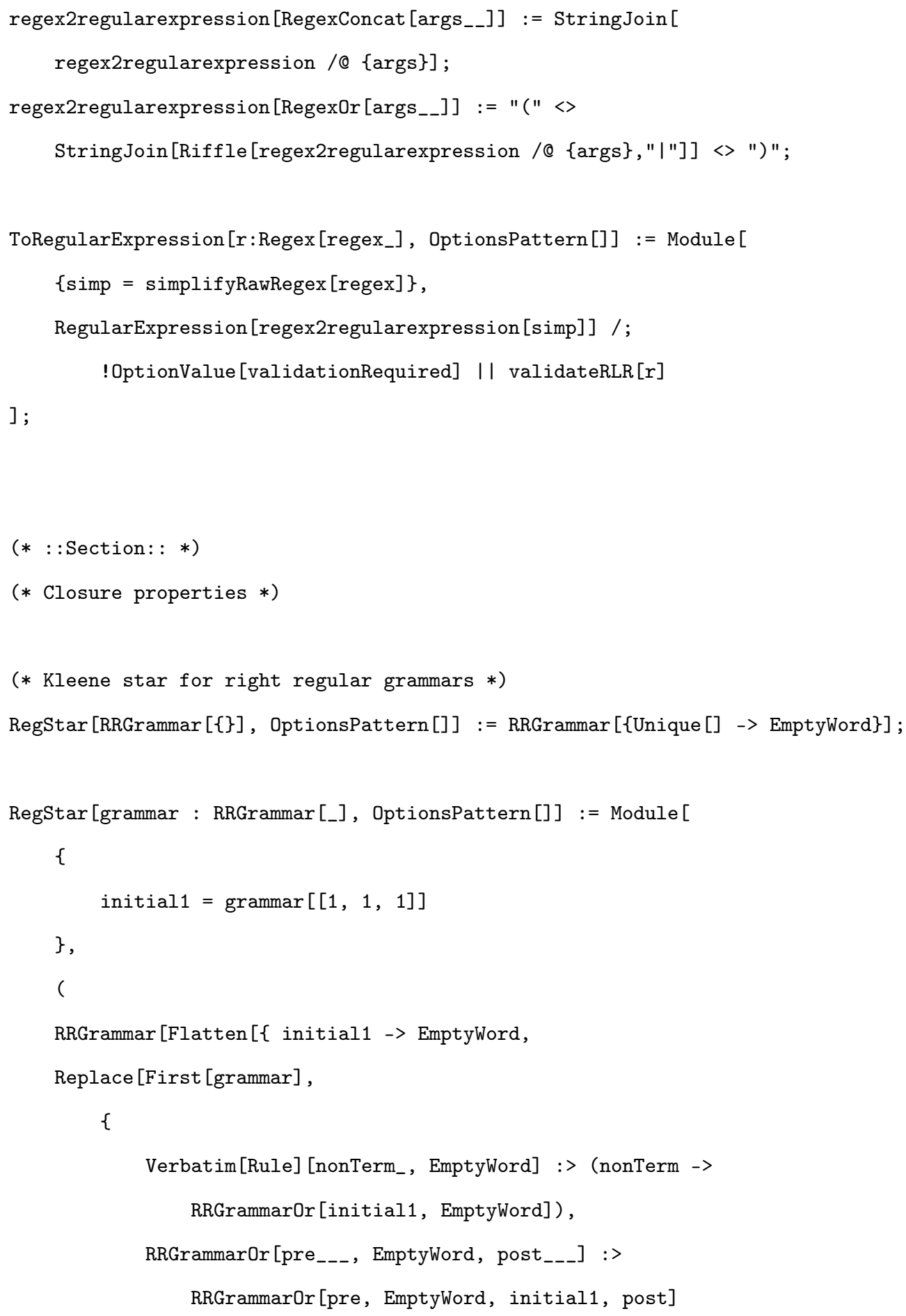


\},

Infinity

]\}] ]

)/; !OptionValue[validationRequired] II validateRLR [grammar]

];

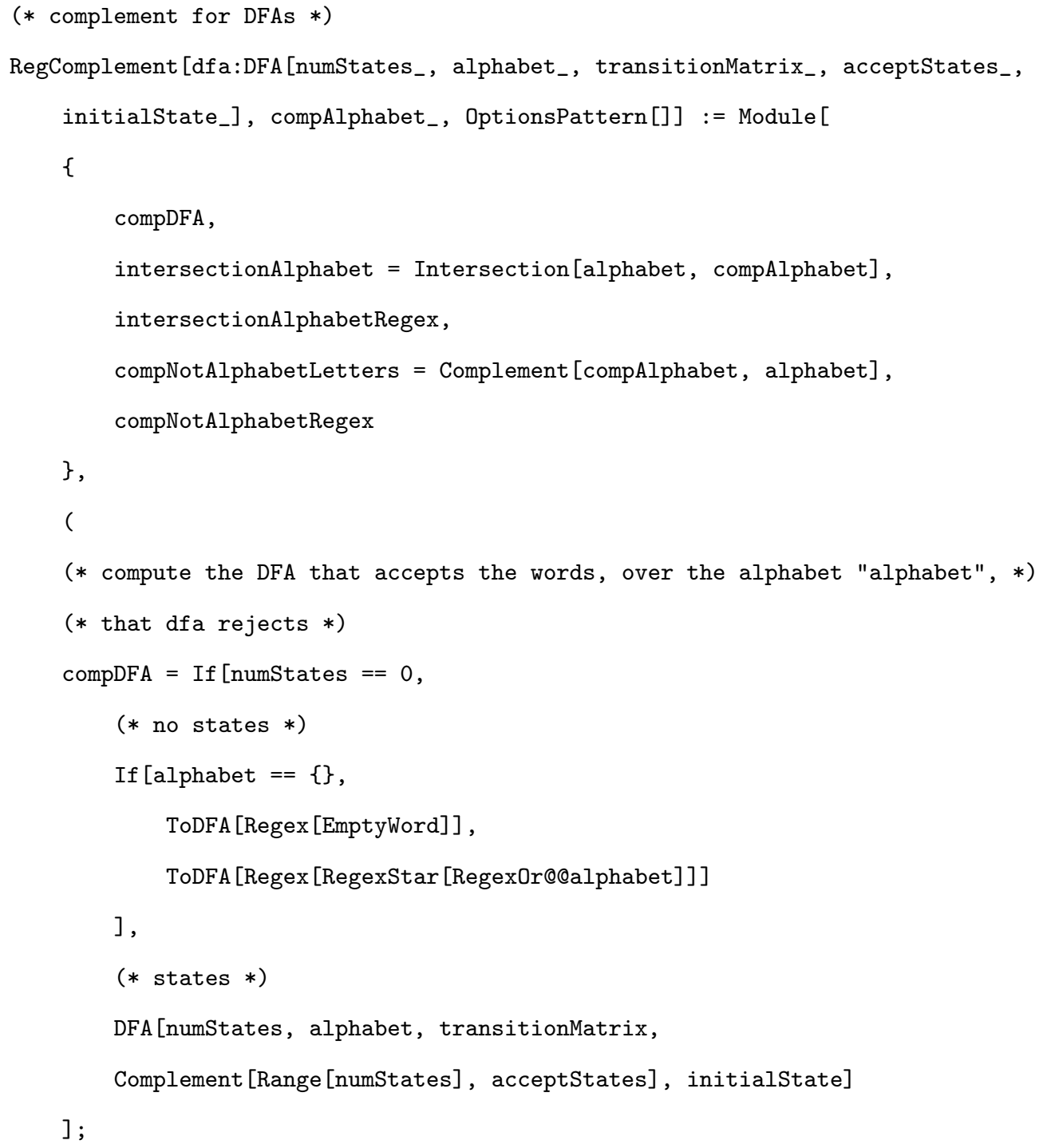




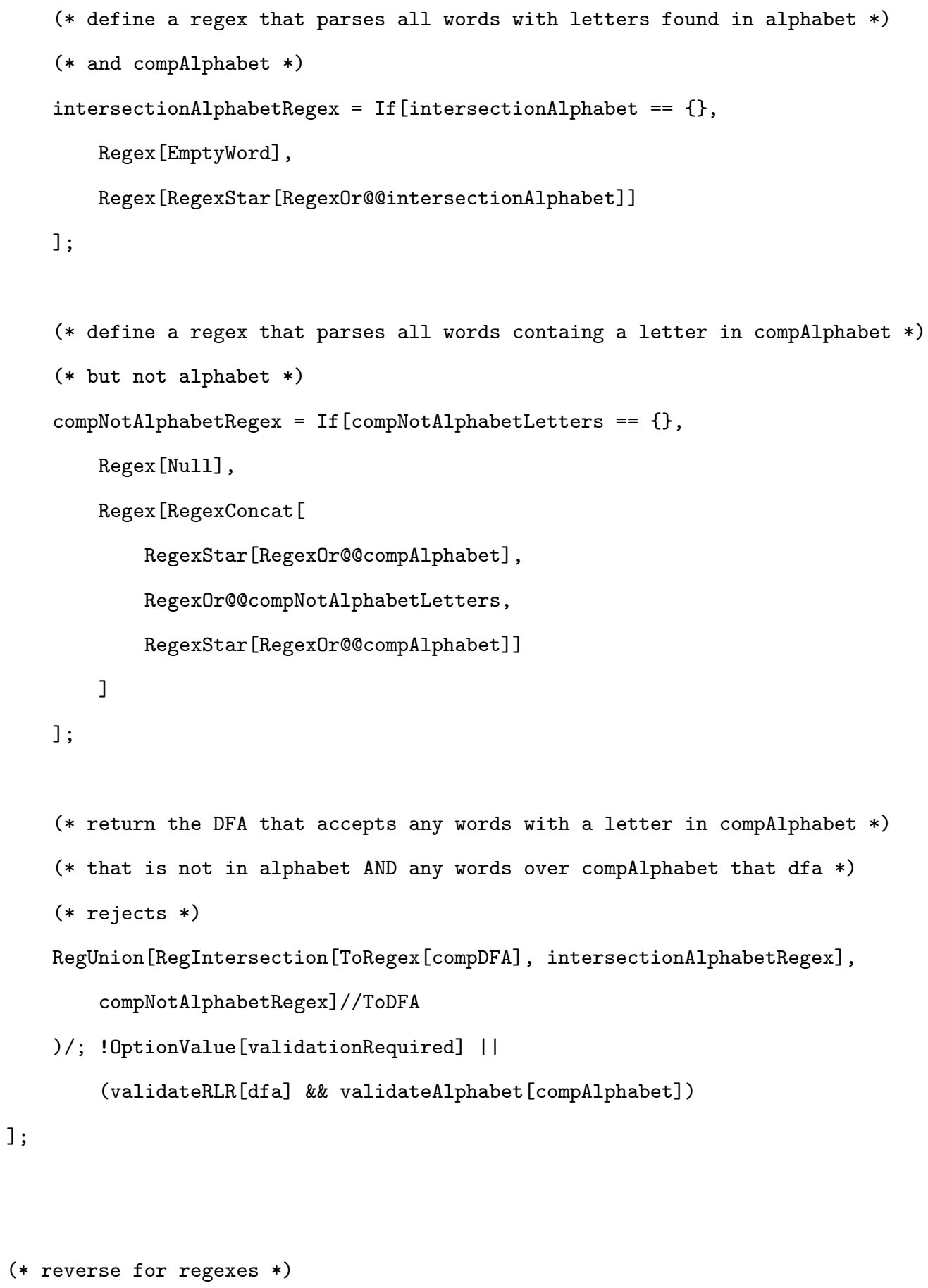




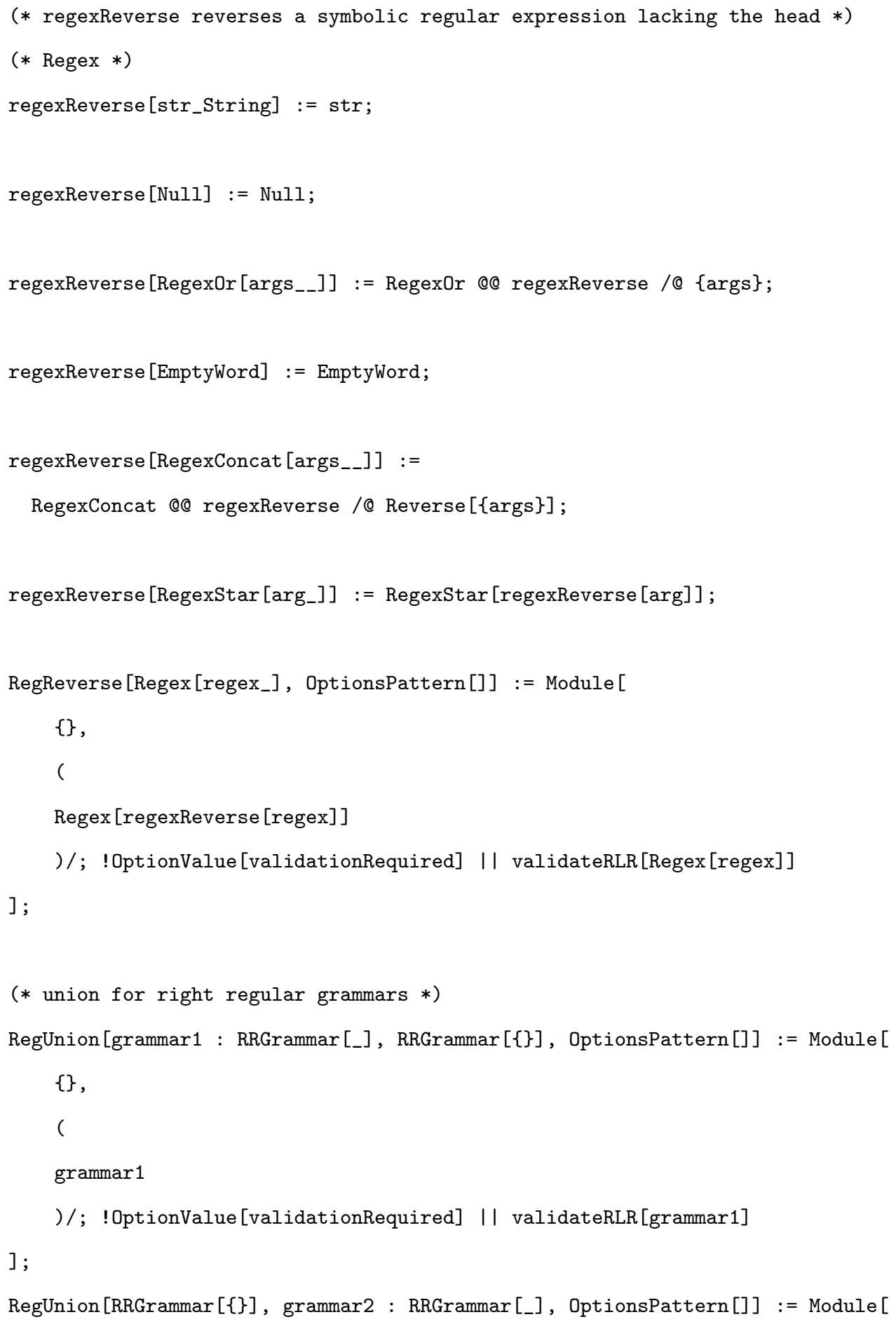




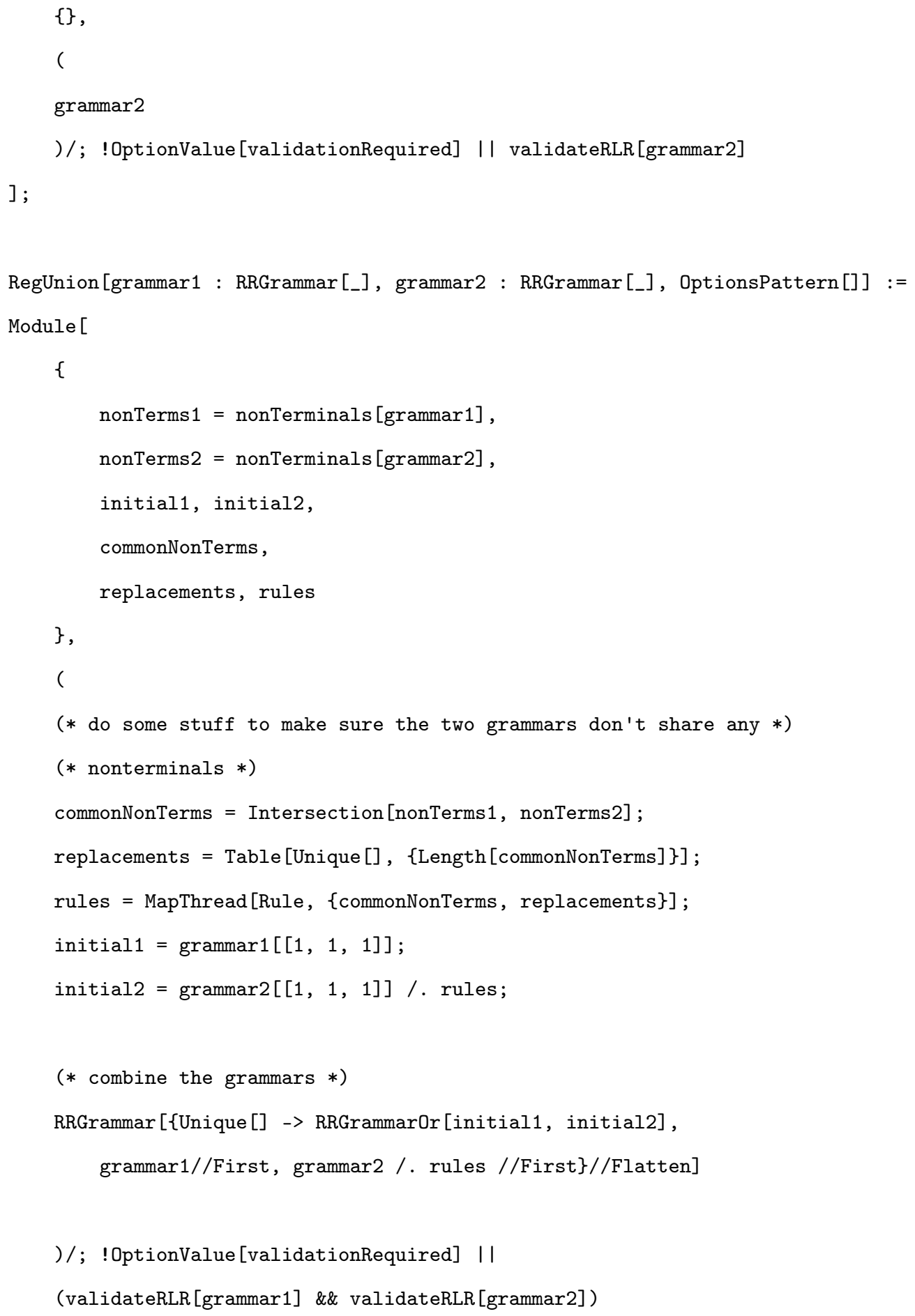


(* concatenation for right regular grammars *)

RegConcat [grammar1 : RRGrammar [_], RRGrammar [\{\}], OptionsPattern[]] := Module [

\{\} ,

RRGrammar $[\{\}]$ /; ! OptionValue[validationRequired] II

validateRLR [grammar1]

];

RegConcat [grammar1 : RRGrammar[_], grammar2 : RRGrammar [_], OptionsPattern[]] := Module [

\{

nonTerms1 = nonTerminals [grammar1],

nonTerms 2 = nonTerminals [grammar2],

initial2,

commonNonTerms,

replacements, rules

\} ,

(

(* do some stuff to make sure the two grammars don't share any $*$ )

(* nonterminals *)

commonNonTerms $=$ Intersection[nonTerms1, nonTerms2];

replacements $=$ Table [Unique [], \{Length [commonNonTerms] $\}$;

rules $=$ MapThread [Rule, \{commonNonTerms, replacements $\}$;

initial2 $=\operatorname{grammar} 2[[1,1,1]] /$ rules;

RRGrammar [Flatten $[\{$

First [grammar1] /. \{

Verbatim[Rule] [nonTerm_, EmptyWord] :>

(nonTerm -> initial2), 


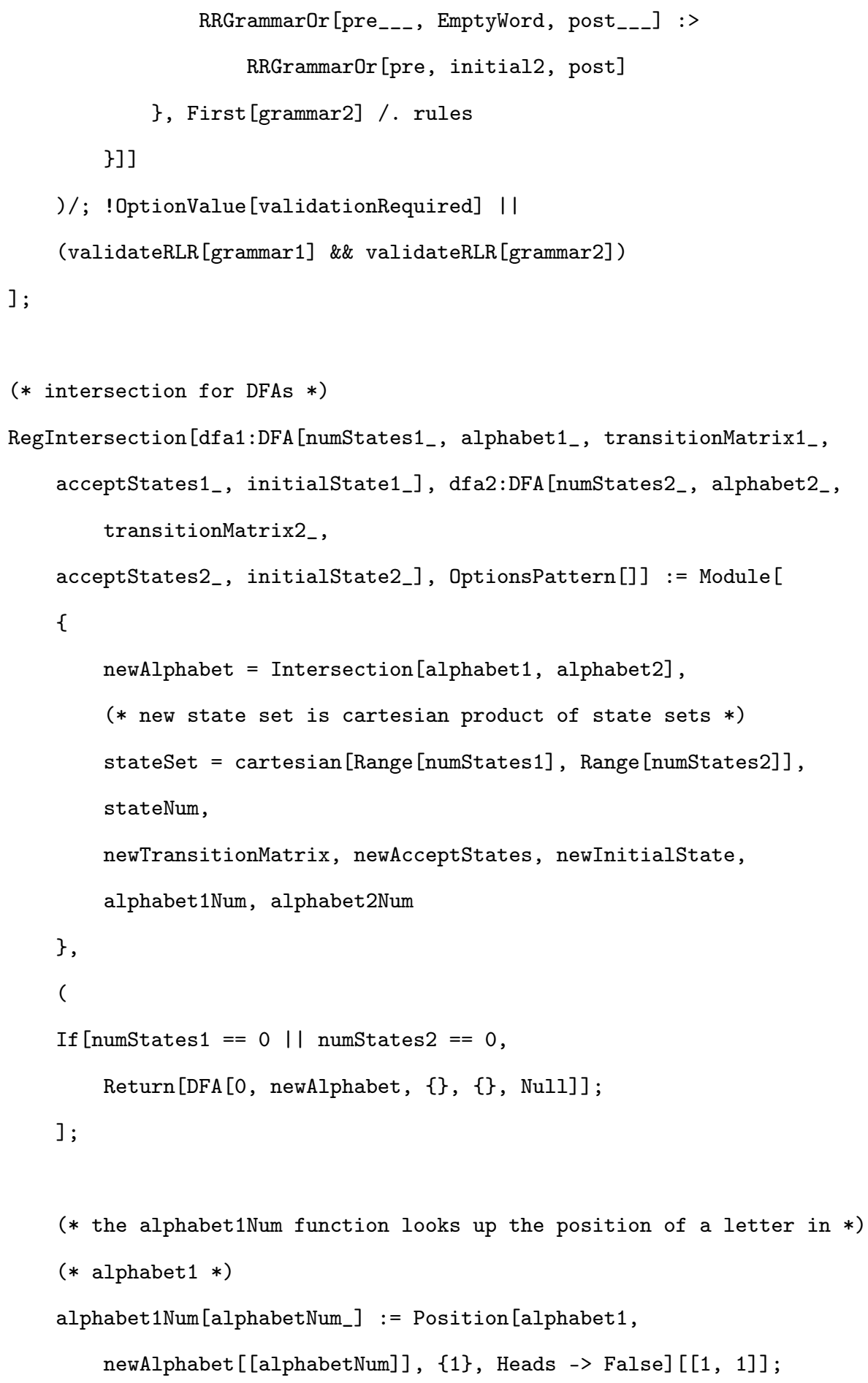




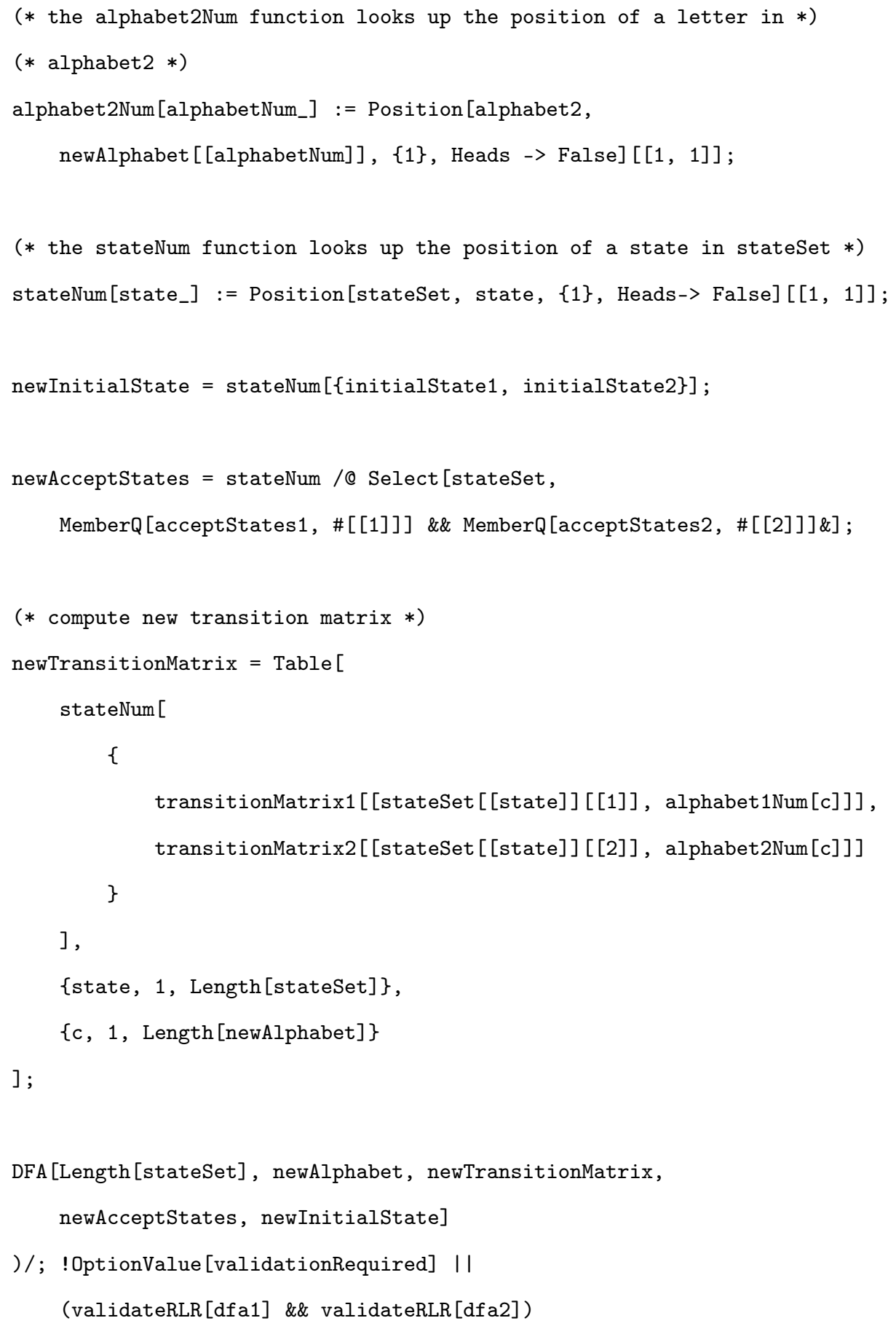




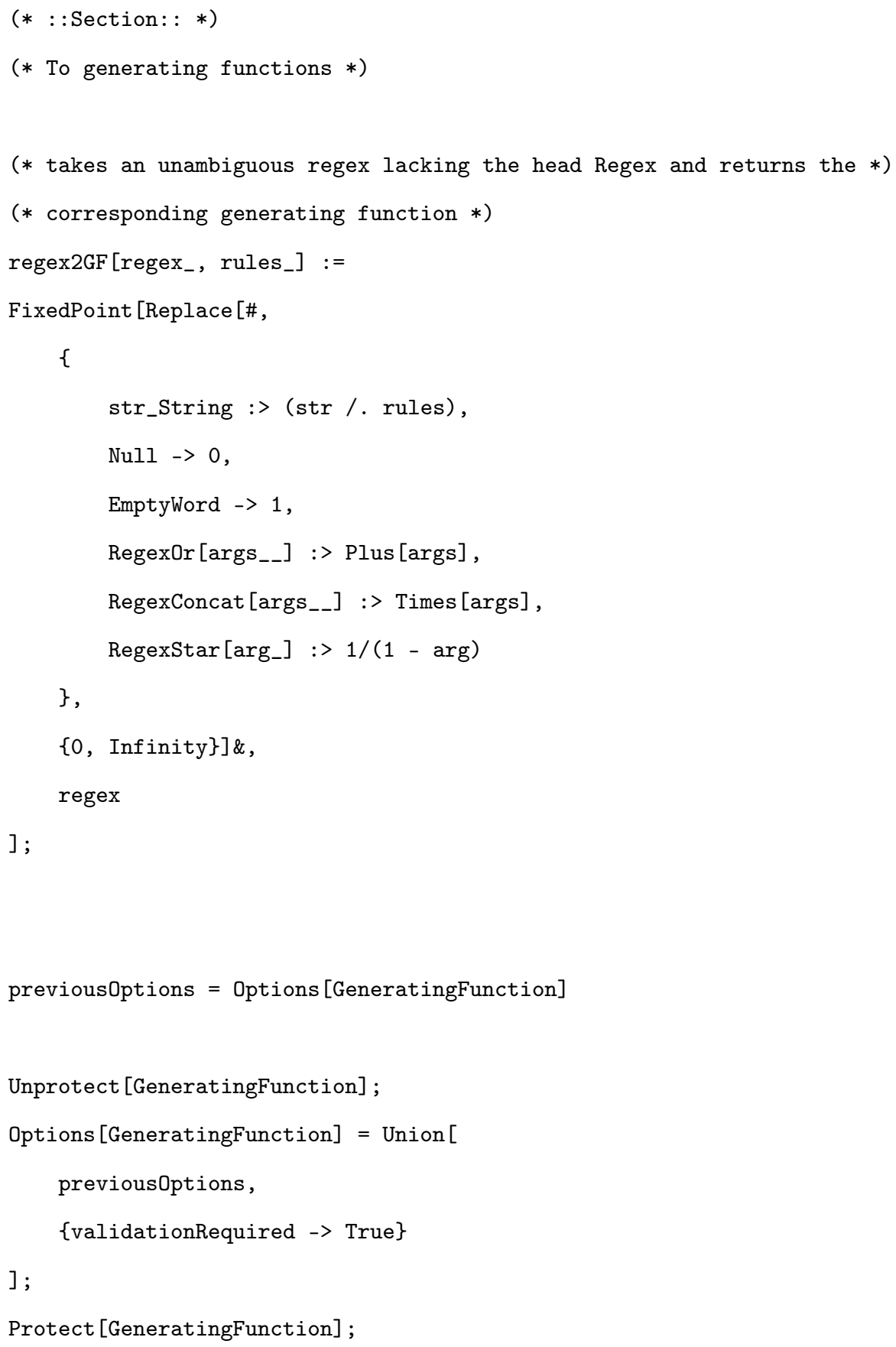




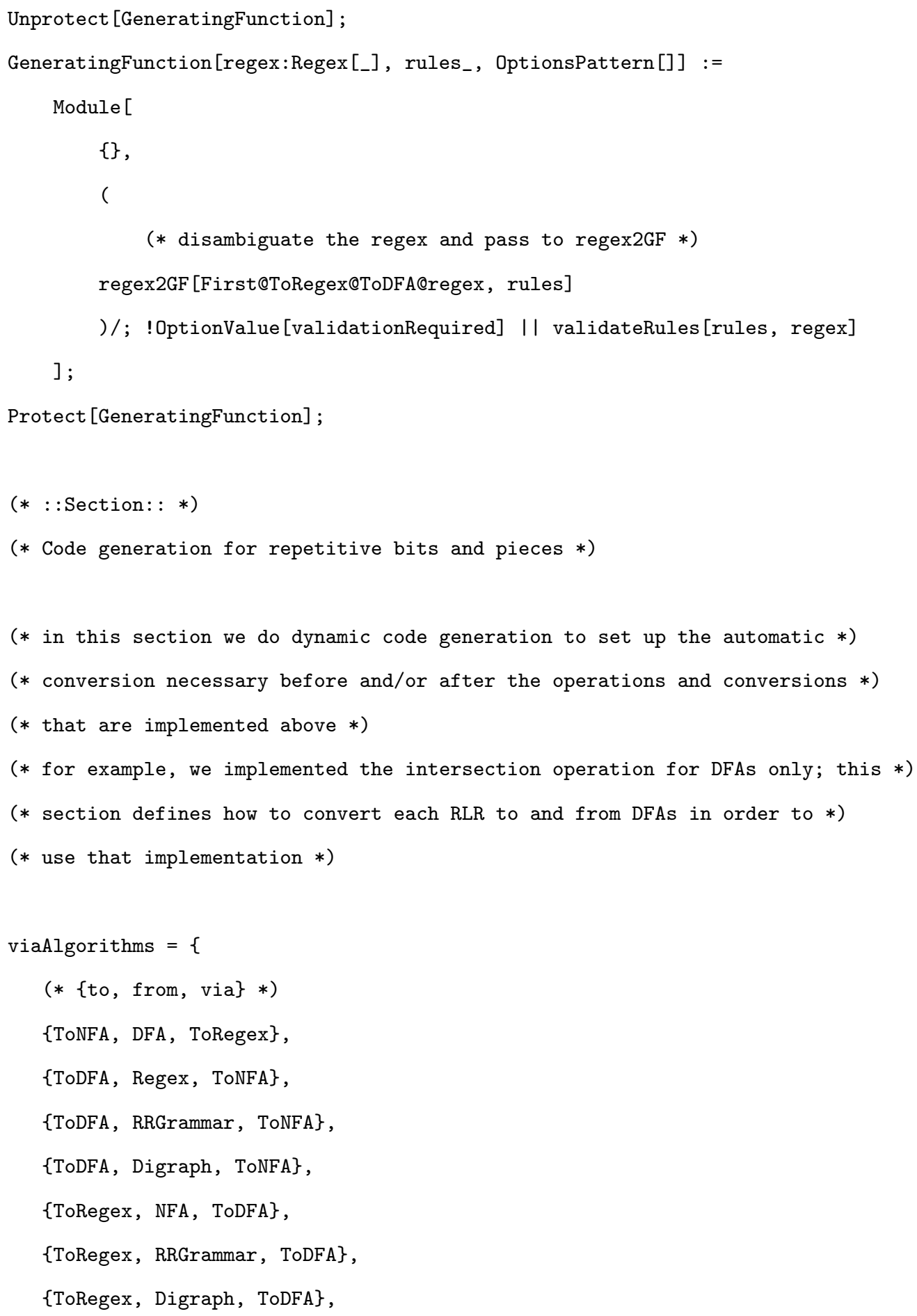




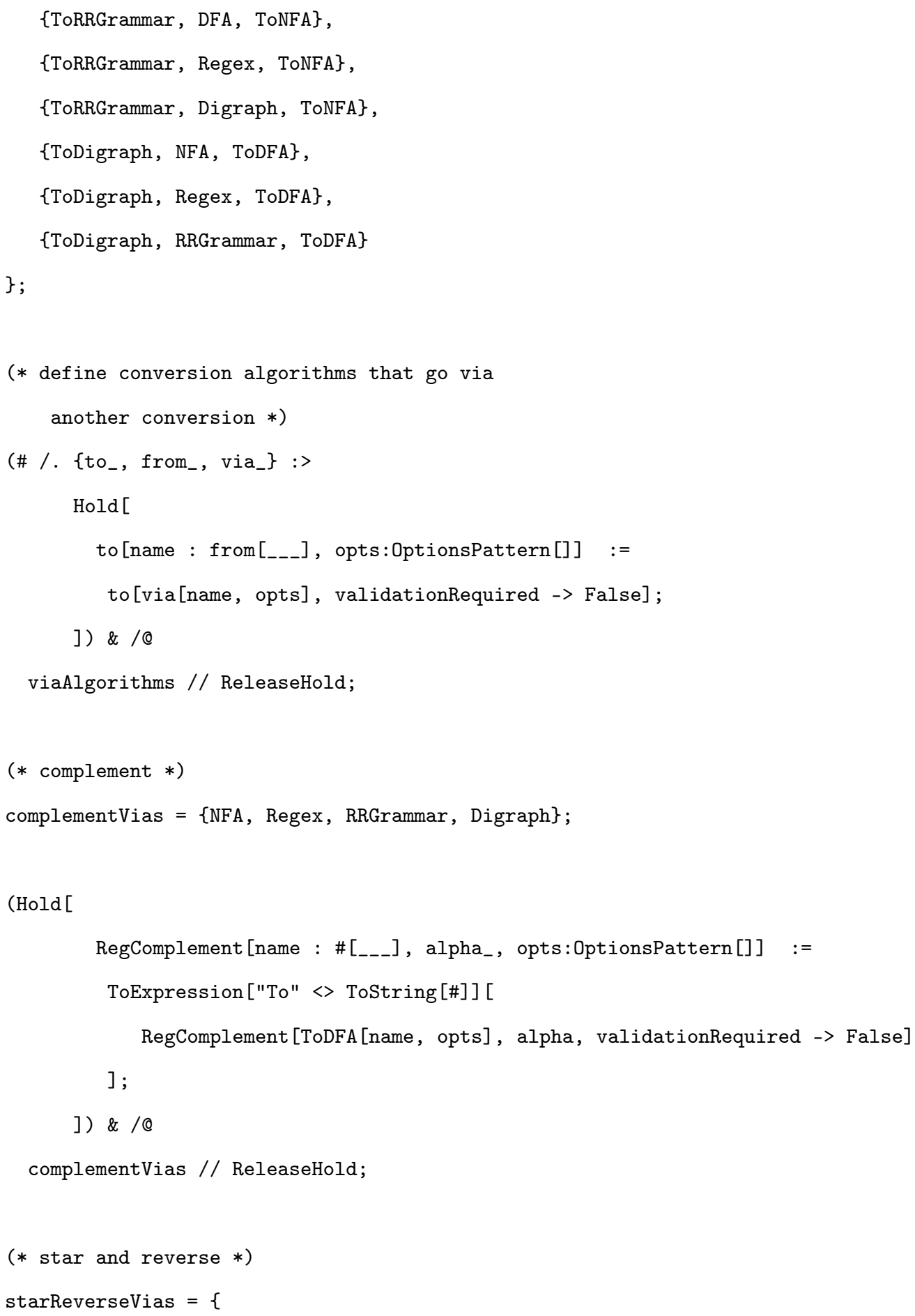




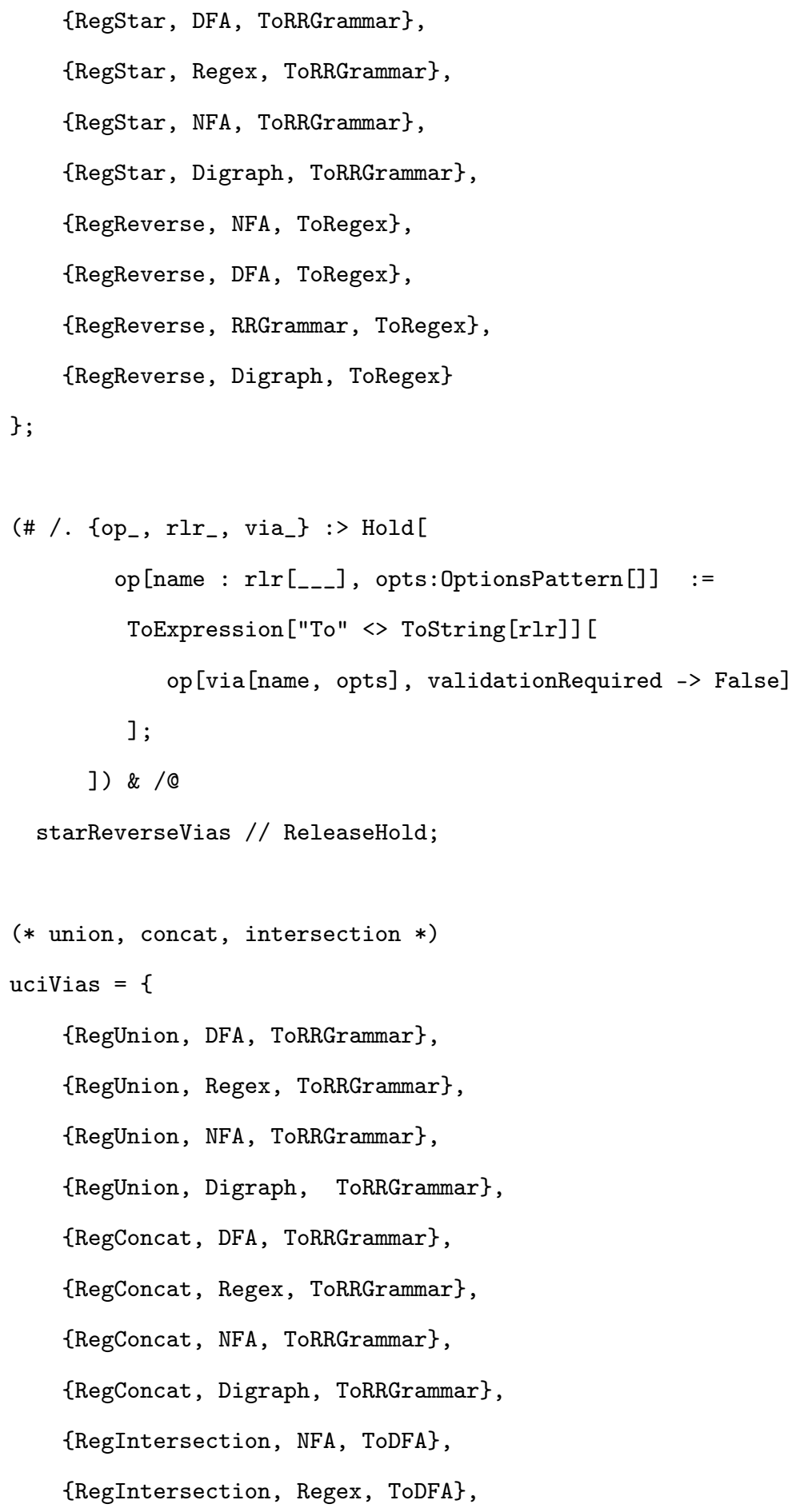




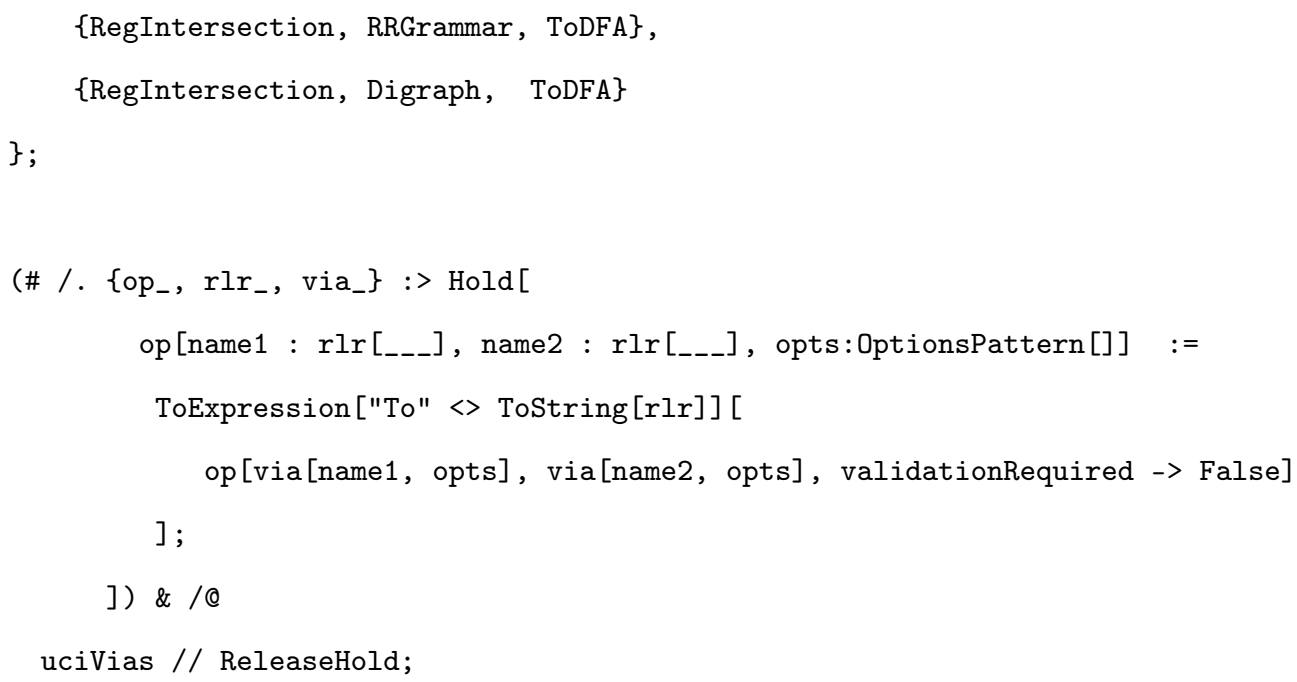


EndPackage []

\section{GFeqn2coefs.m}

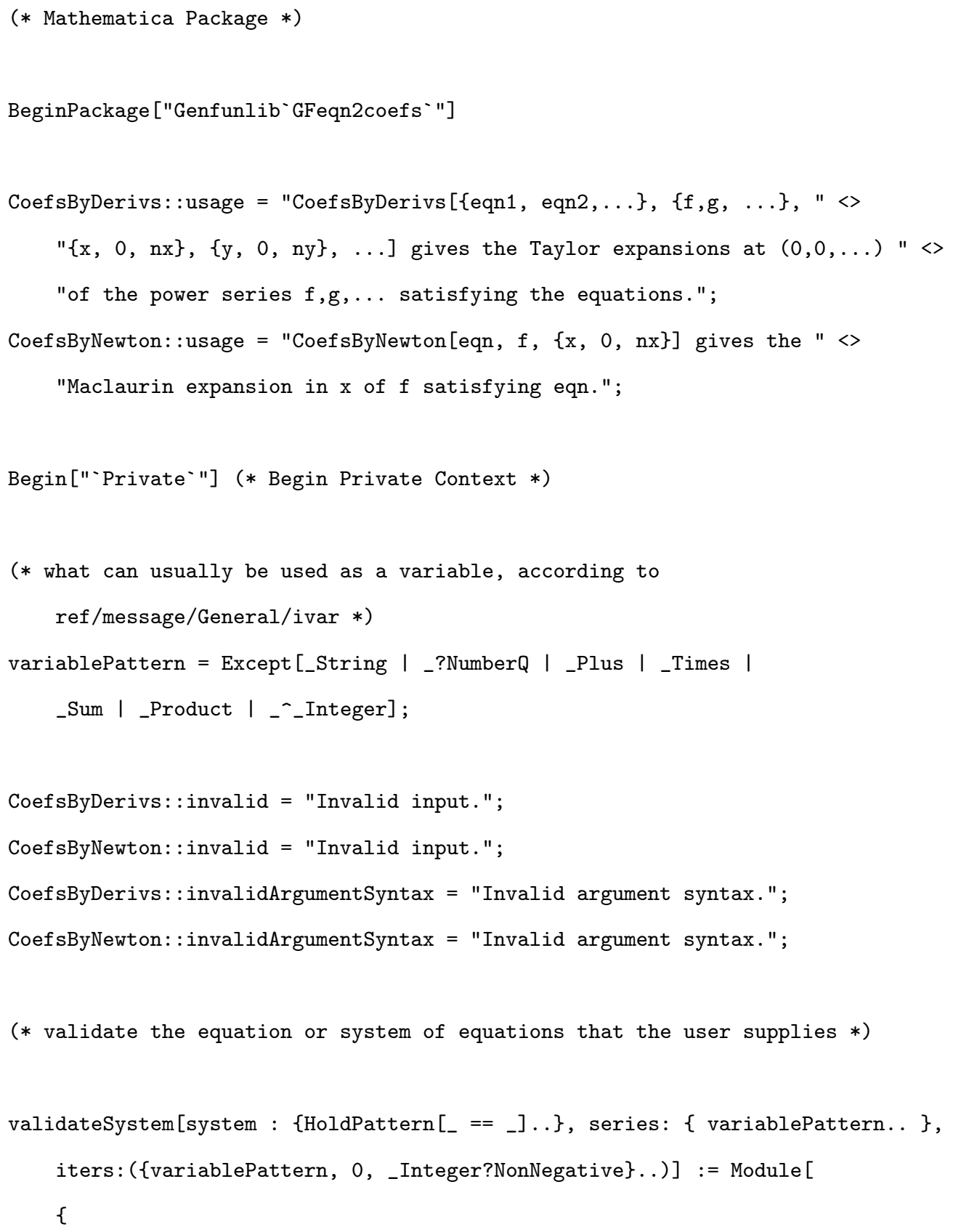




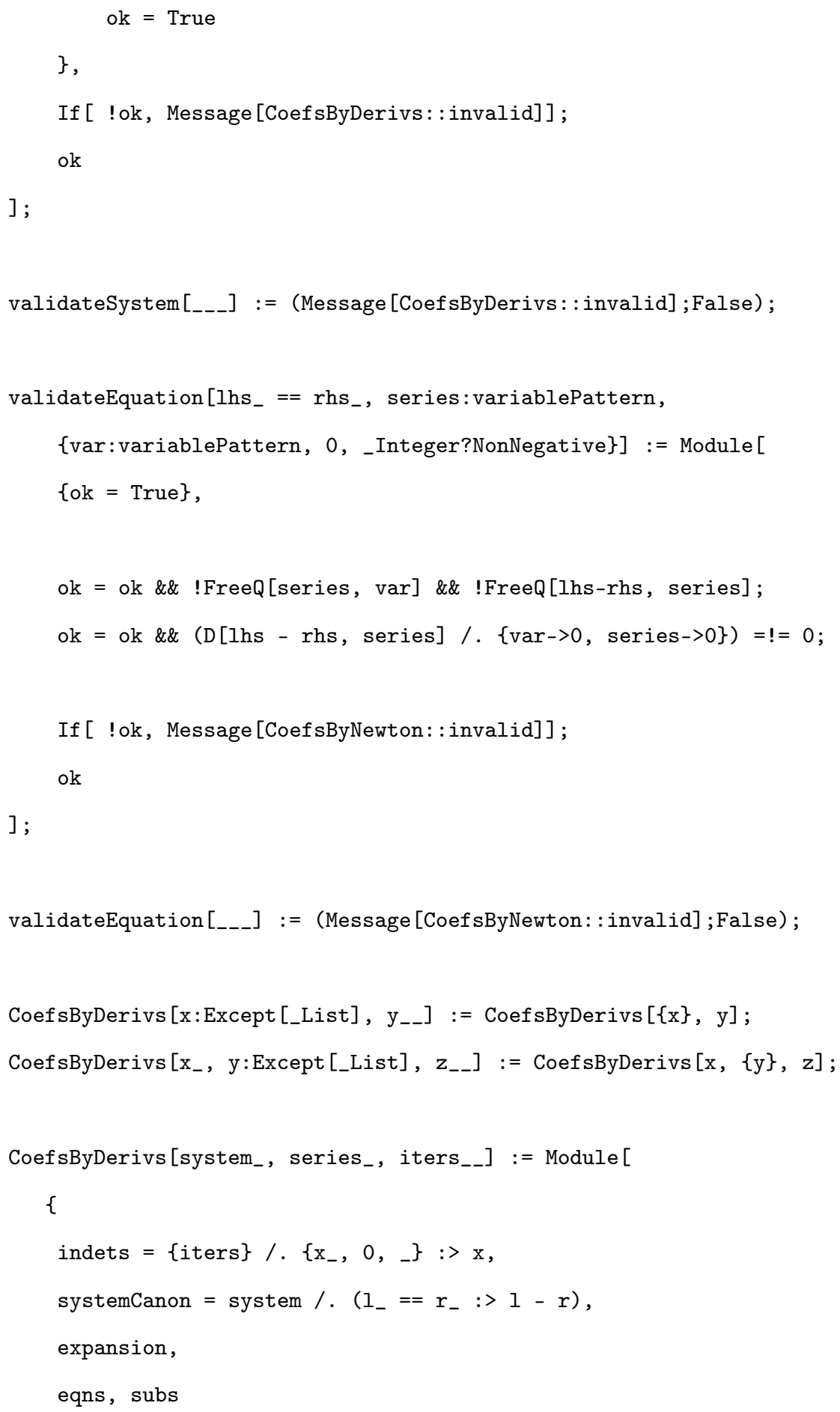









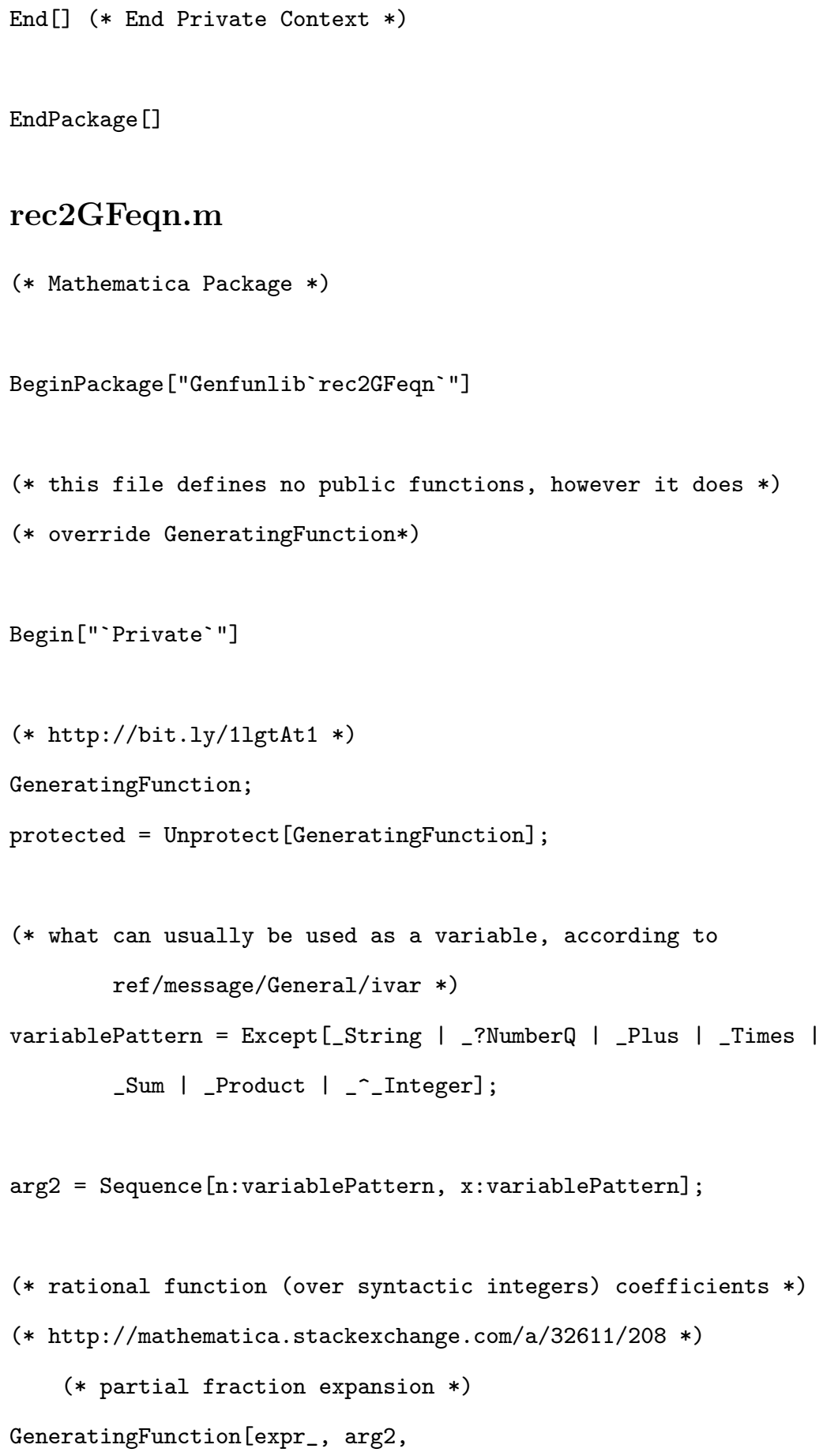




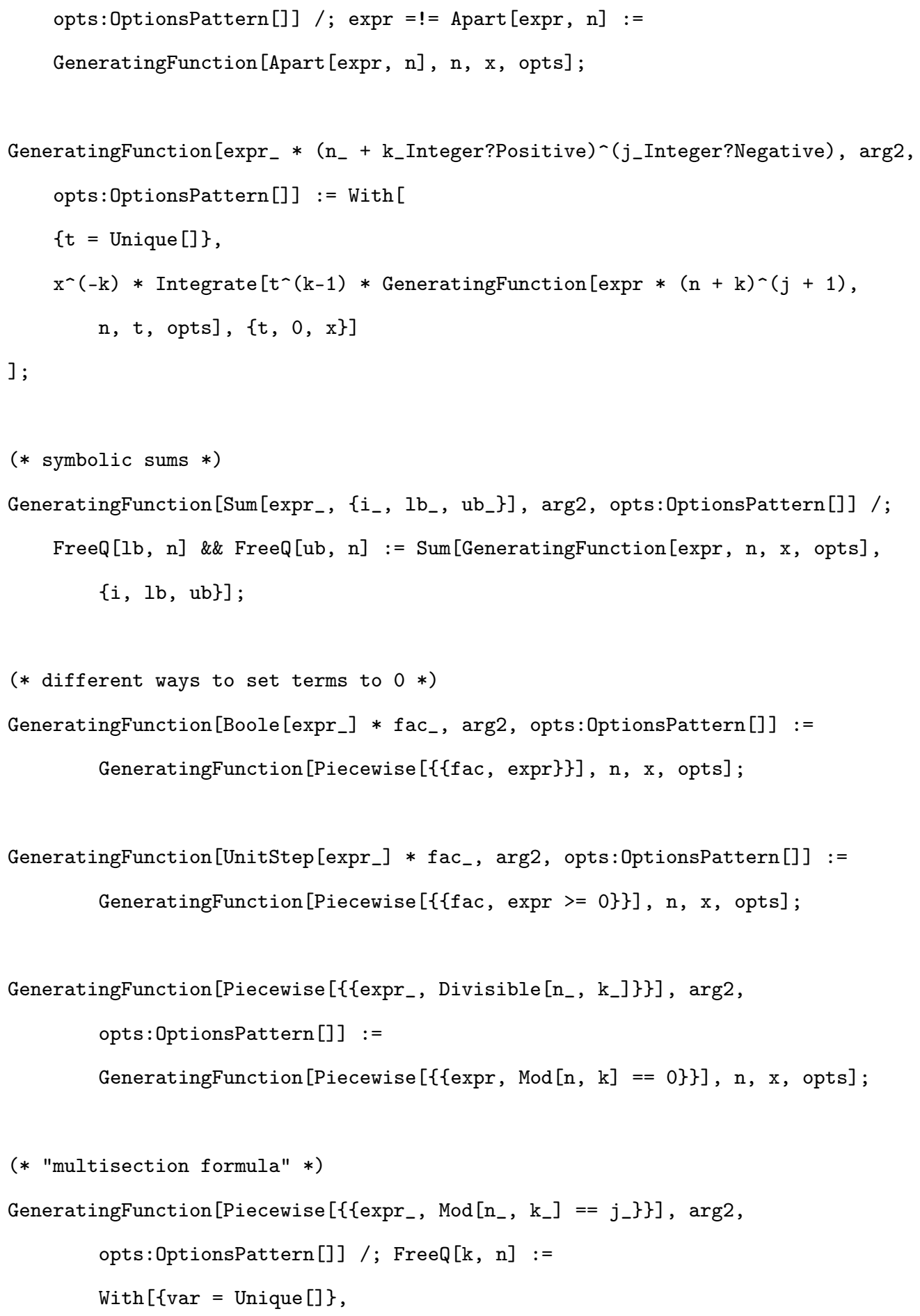




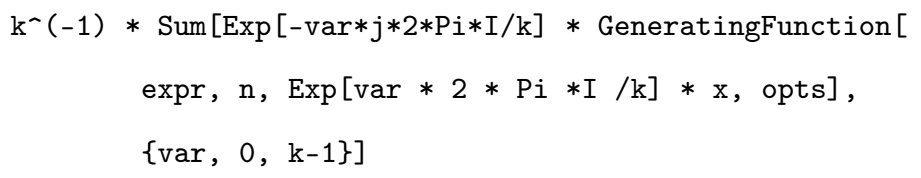

\section{SymbolicMethod.m}

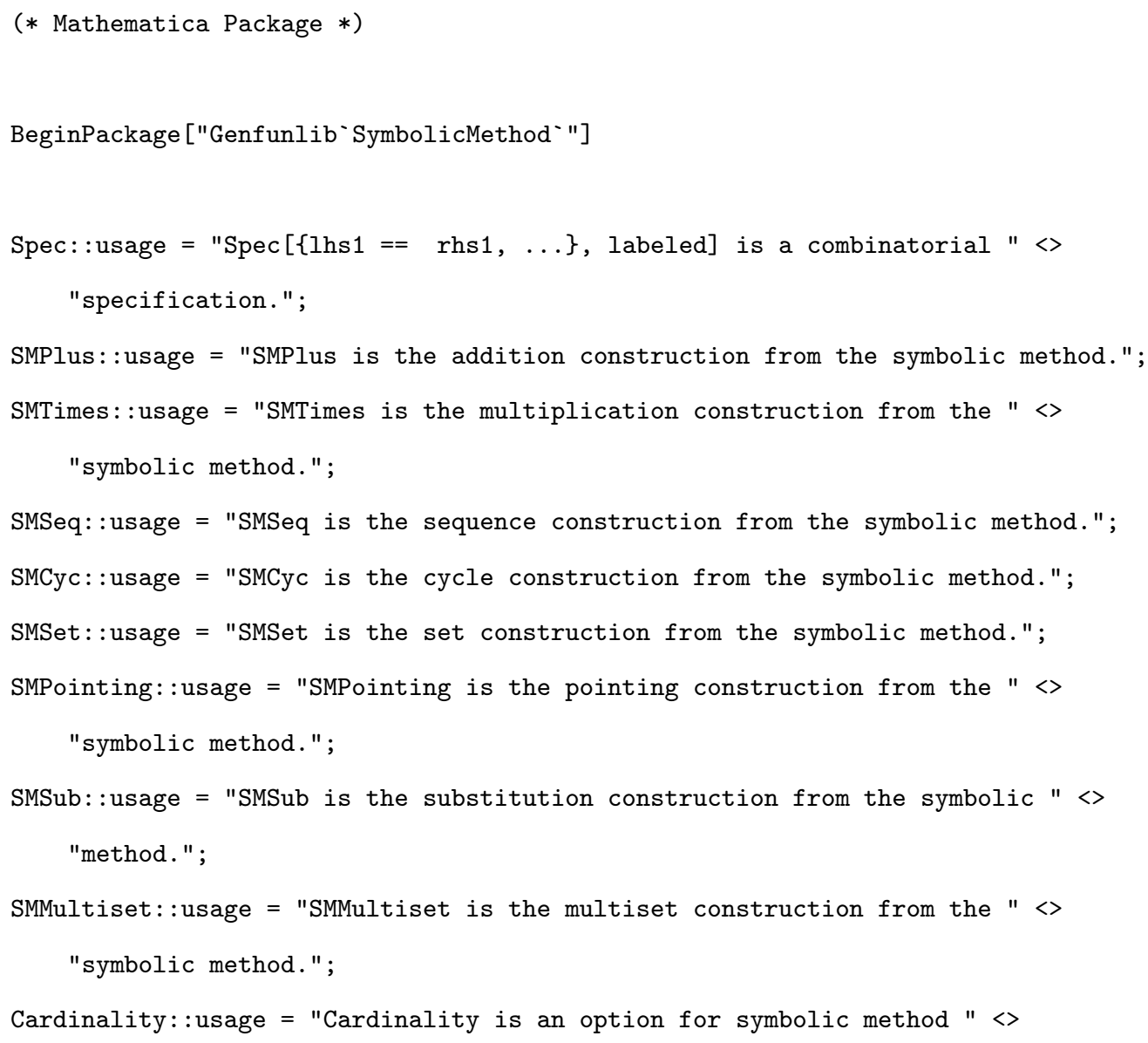




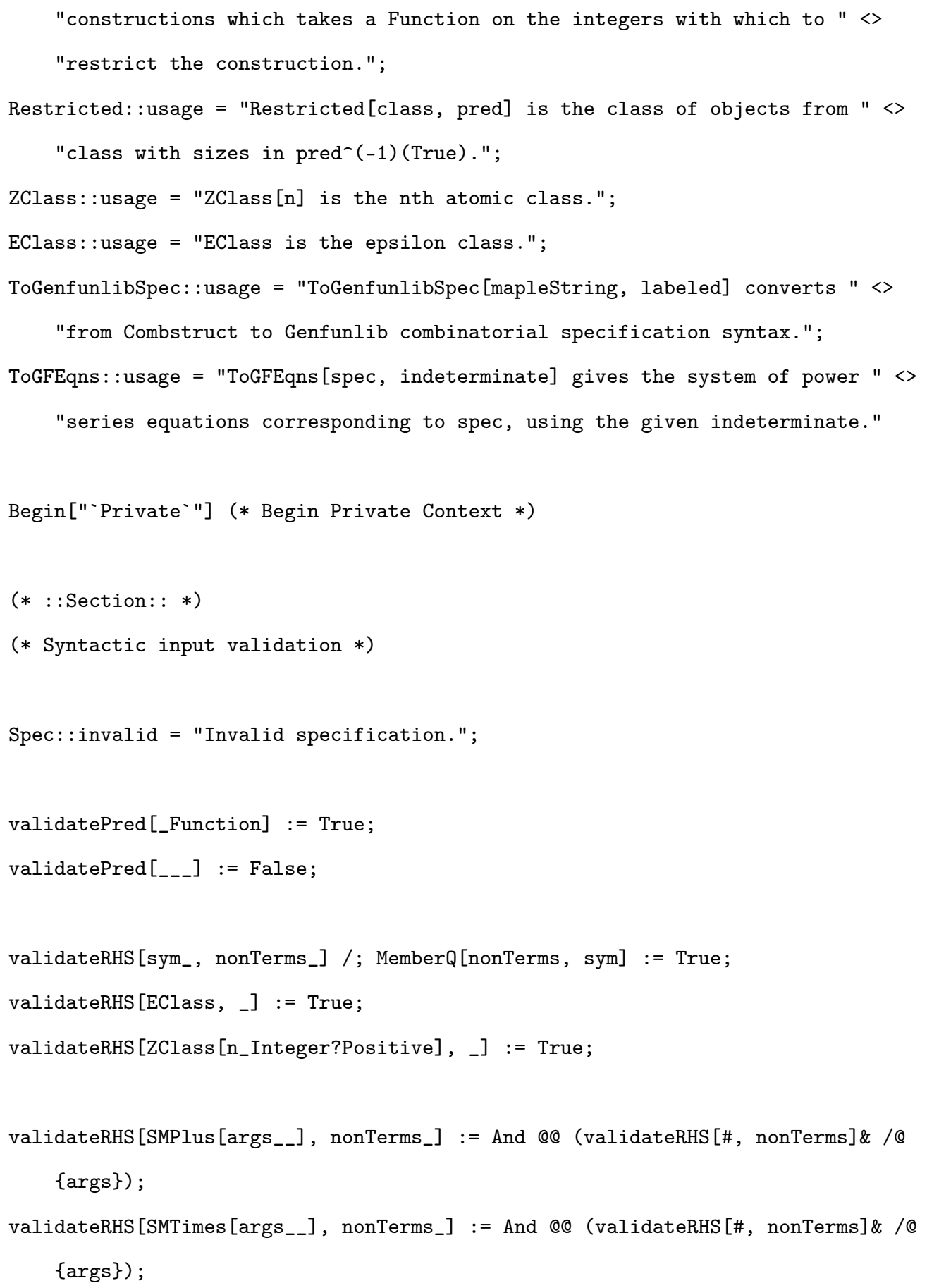




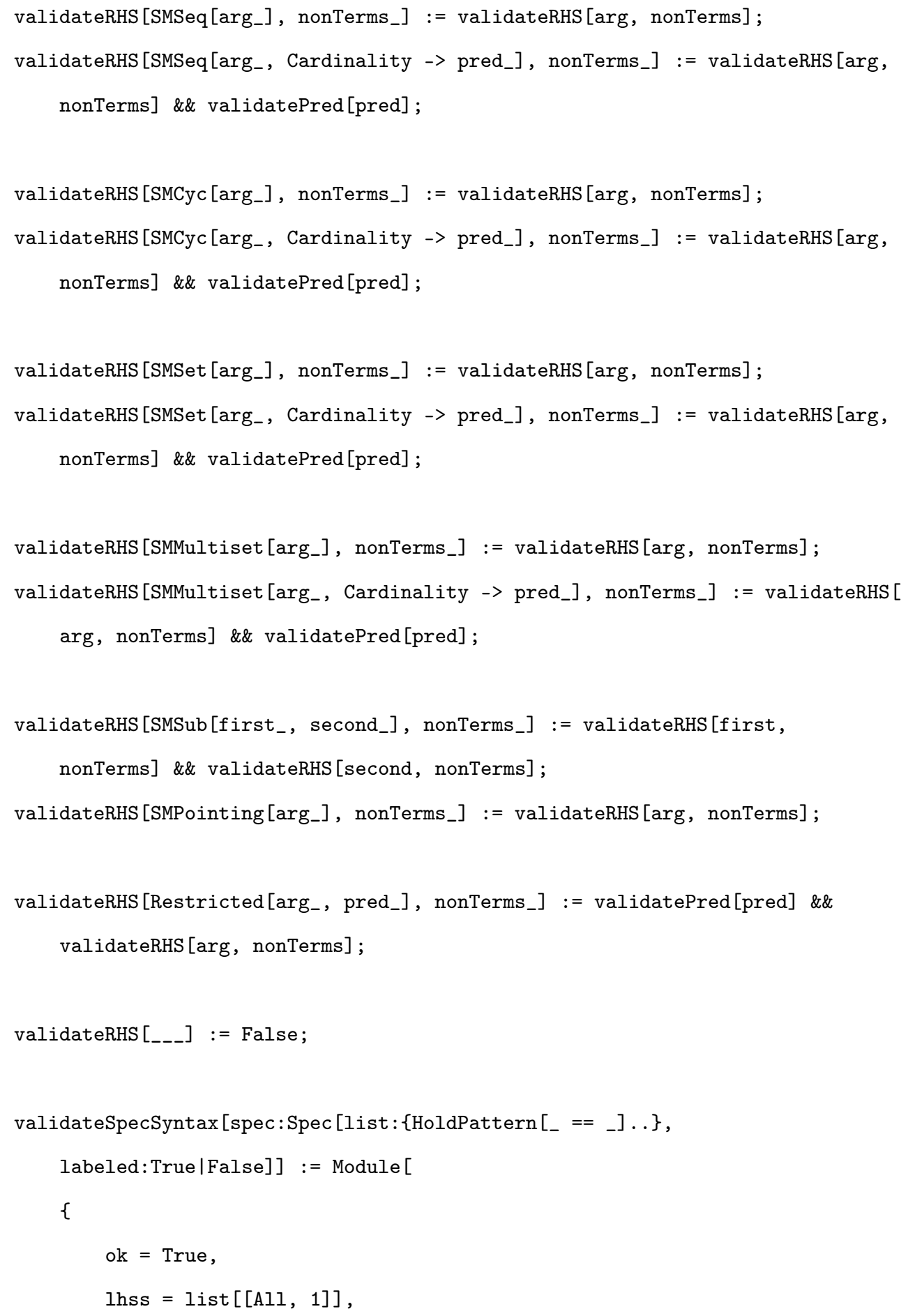




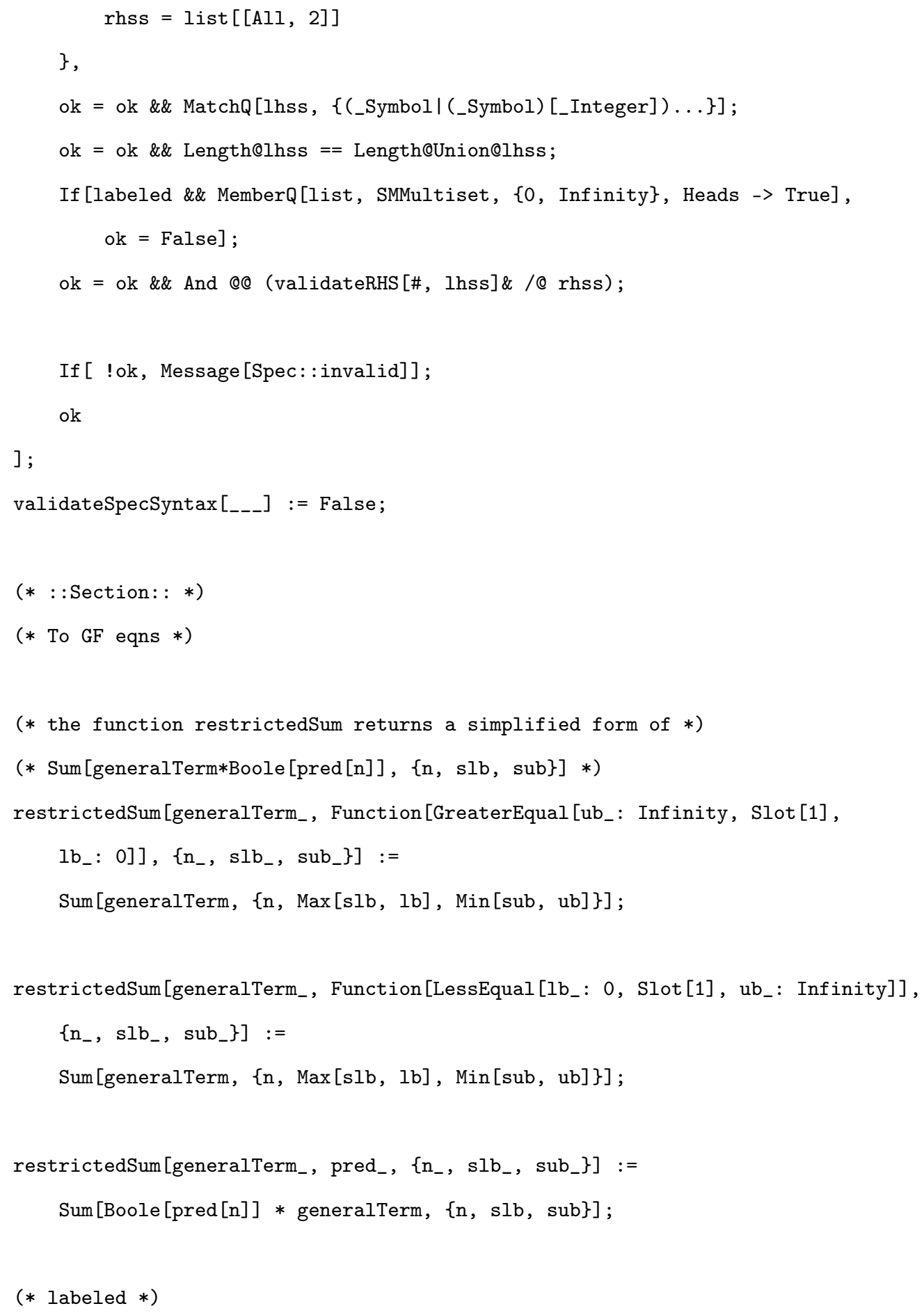




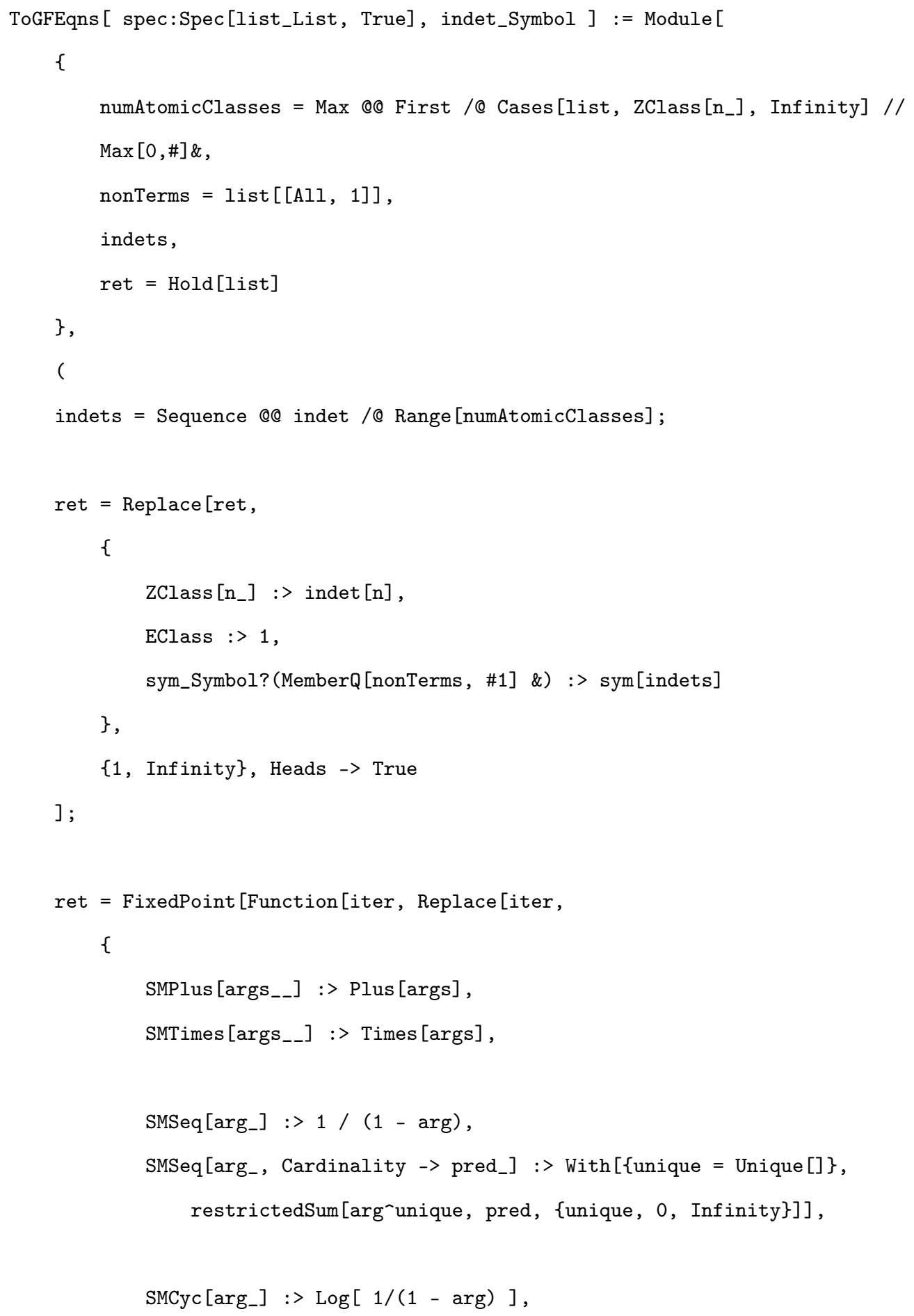




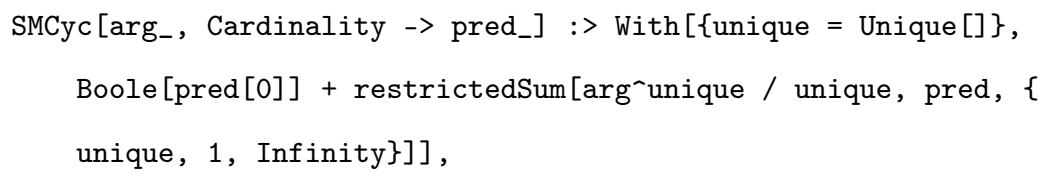




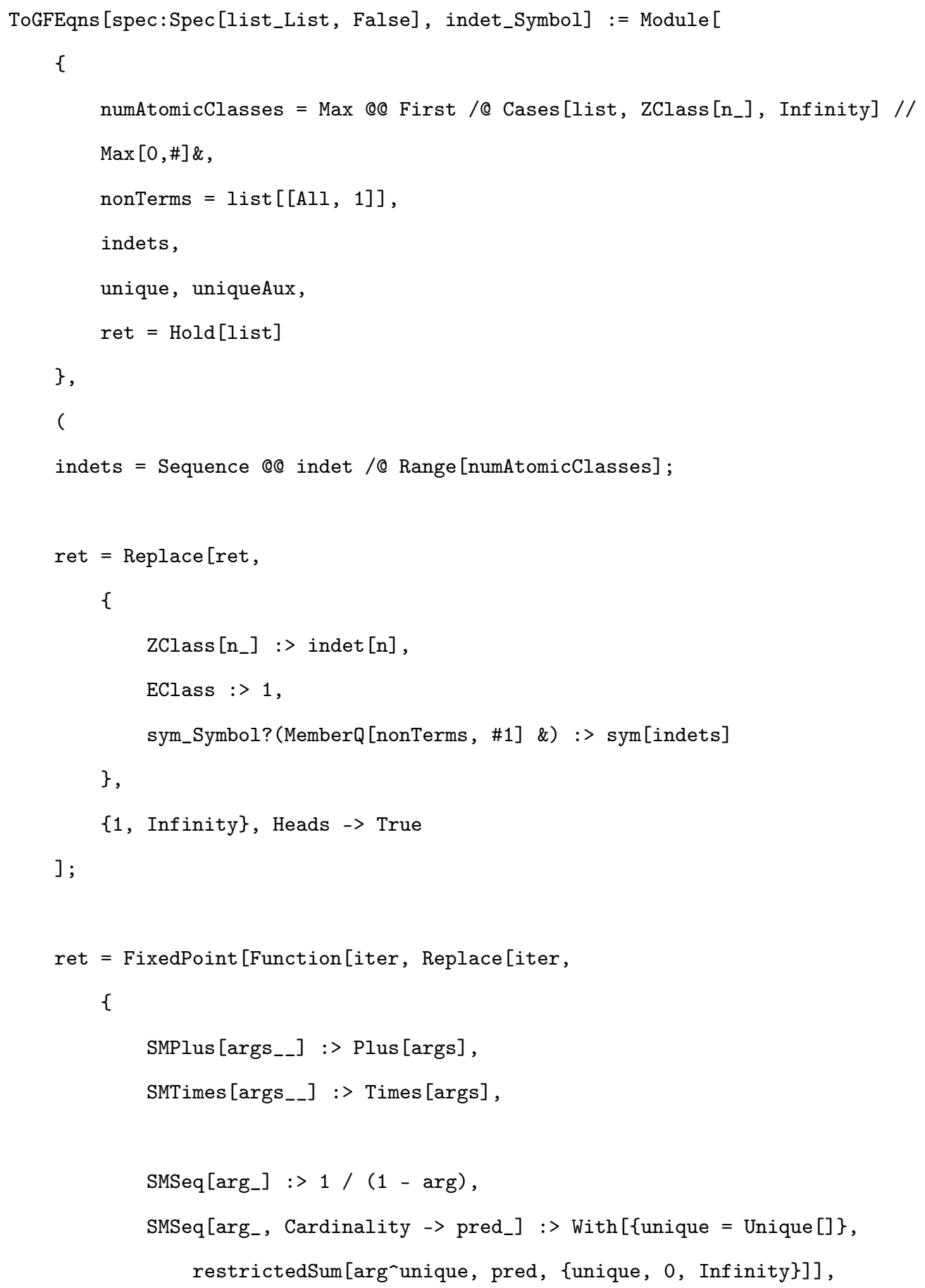




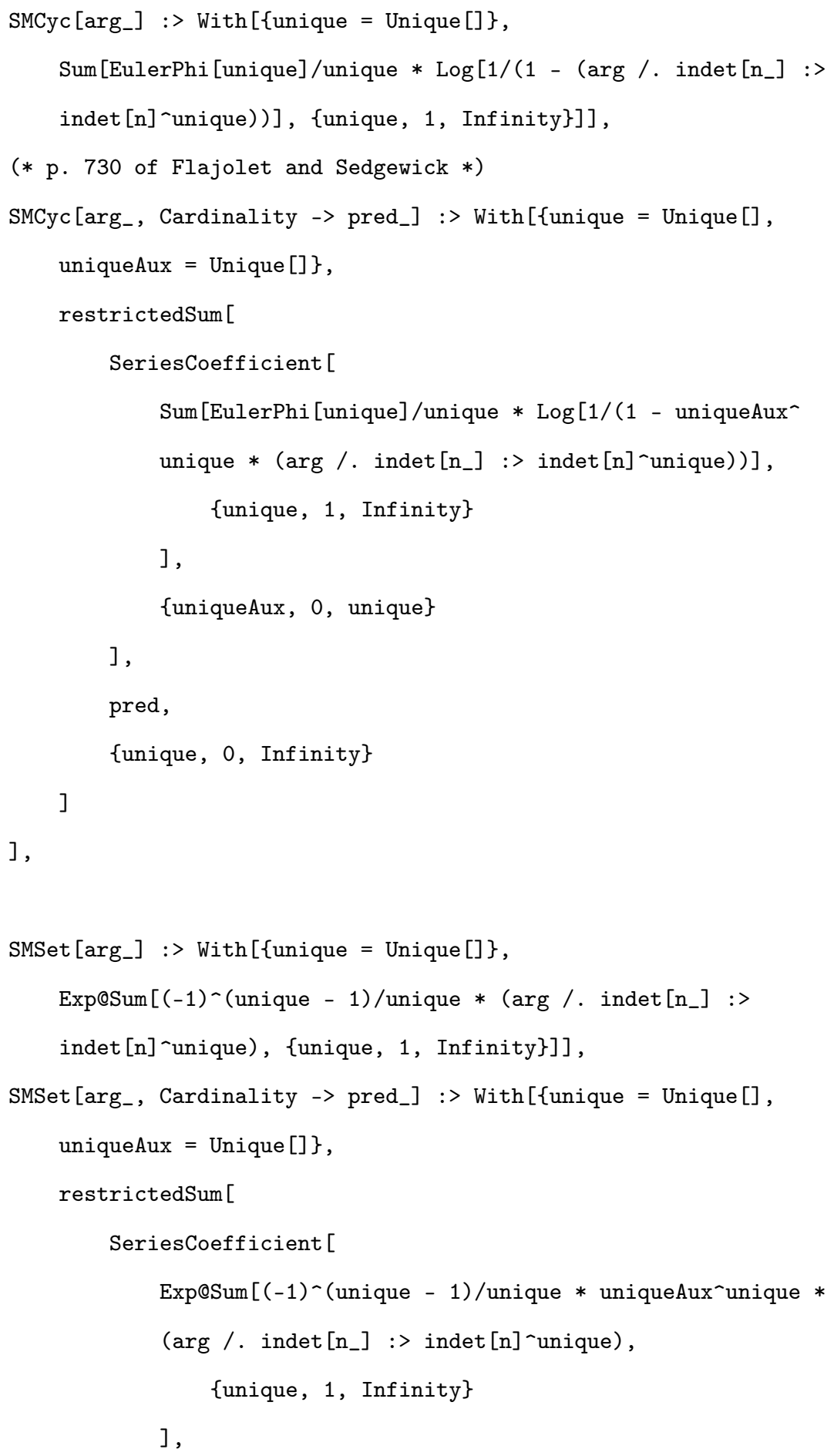




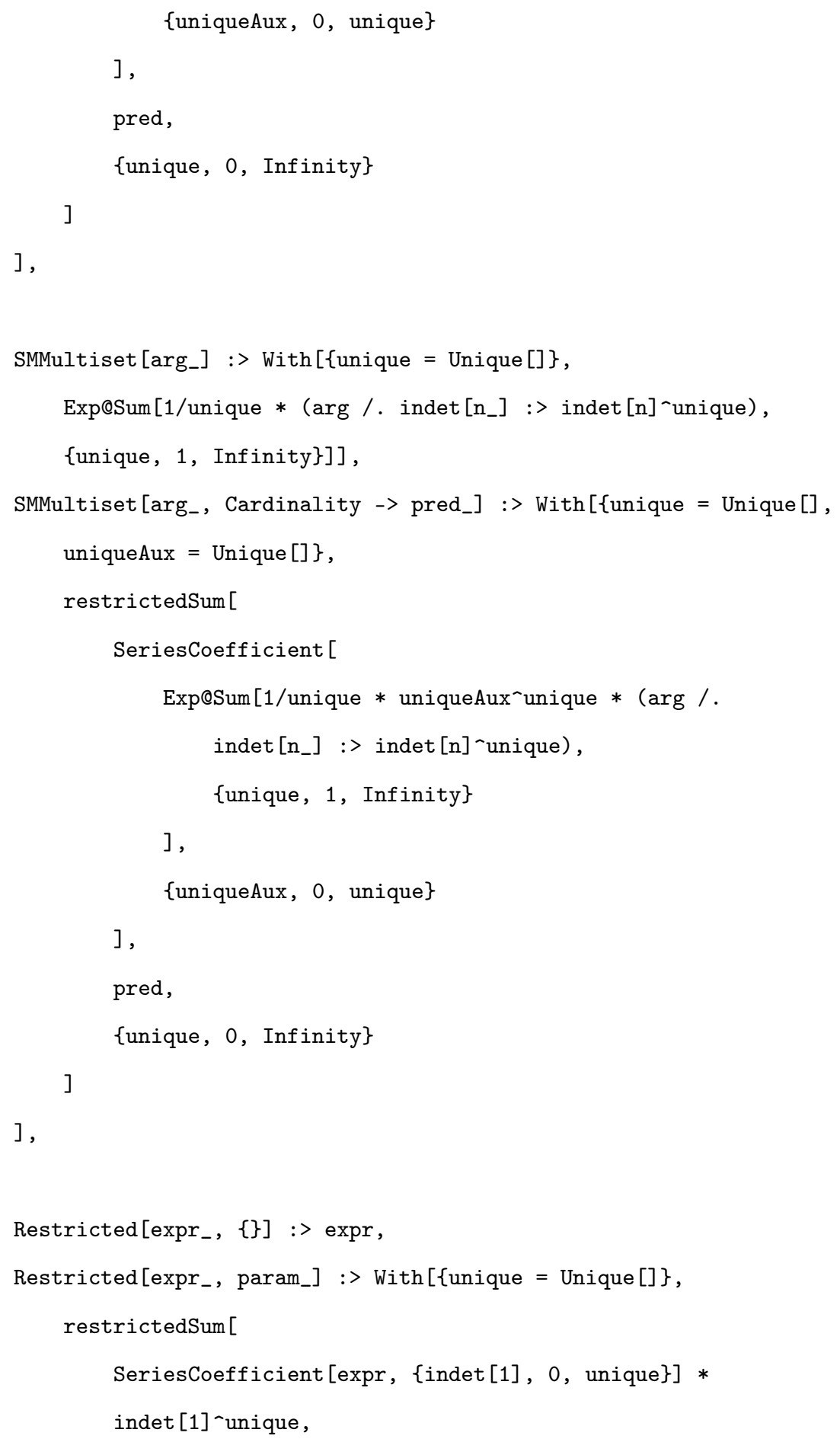


pred, \{unique, 0 , Infinity\}

]],

SMPointing[expr_] :> indet[1] * D[expr, indet[1]],

SMSub [func_, arg_] :> (func /. indet [1] :> arg)

\}, $\{1$, Infinity $\}$, Heads $->$ True

] ], ret];

ReleaseHold [ret]

) /; validateSpecSyntax $[$ spec $]$

];

(*::Section: : *)

(* Combstruct grammar to Genfunlib spec *)

(* this function assumes there are no parentheses except to group function *)

(* arguments, assumes nonterminals are only one character, and *)

(* assumes the grammar is explicit *)

ToGenfunlibSpec[str_String, labeled:(True|False)] := Module[

\{

ret $=\operatorname{str}$

\} ,

ret $=$ StringReplace[ret, c_?UpperCaseQ :> ToLowerCase [c] <> ToLowerCase [c] $]$;

ret $=$ StringReplace $[$ ret, \{

$"="->"=="$,

"(" $\quad$-> "[", ")" -> "]",

"pprod" -> "SMTimes", 


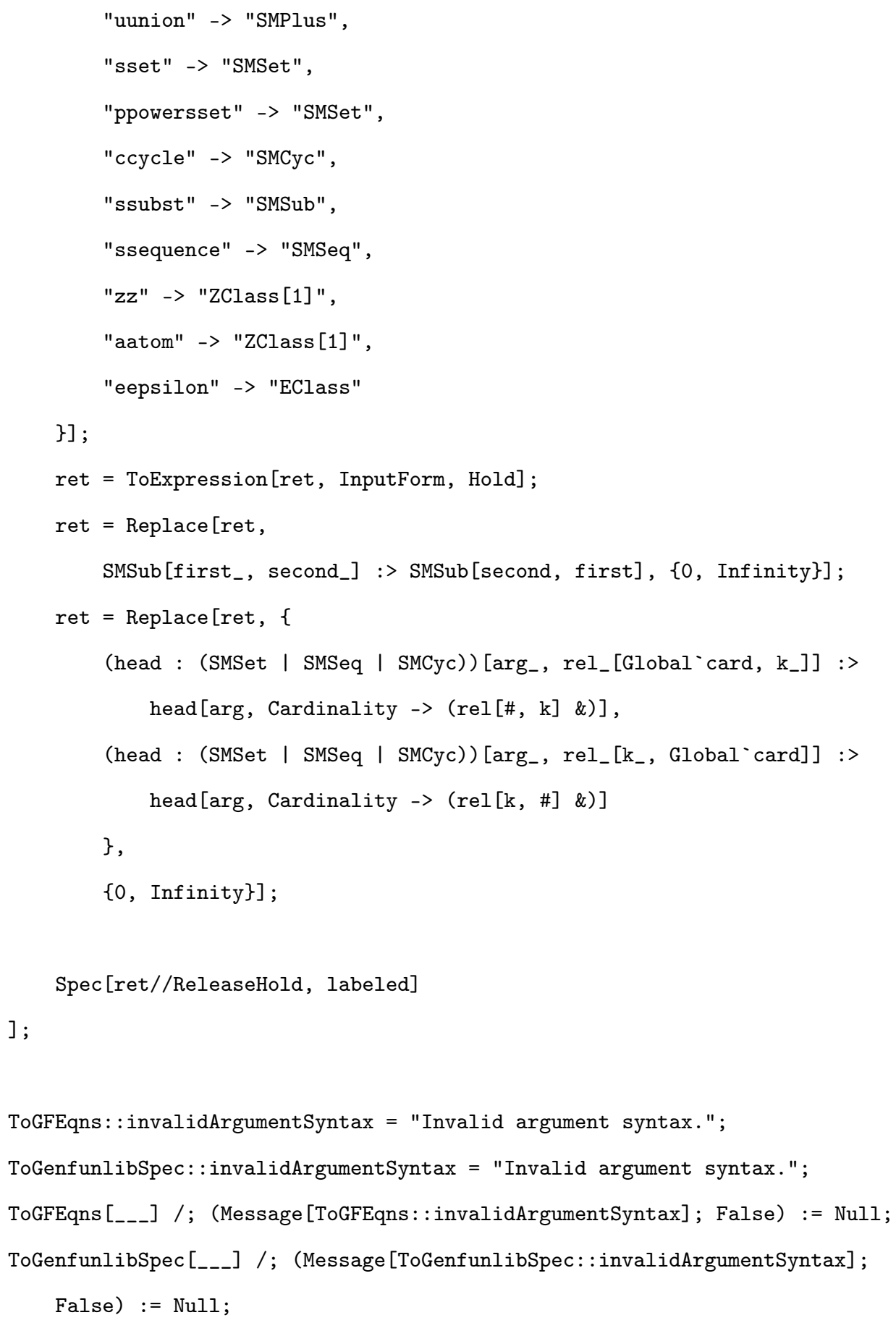


End [] (* End Private Context *)

EndPackage [] 